\title{
High-Energy Higgsstrahlung Cross-Section Suppression in the Minimal Composite Higgs Model
}

\author{
by
}

\author{
Kathryn A. D. Hally
}

\author{
A thesis submitted to the \\ Faculty of Graduate and Postdoctoral Affairs \\ in partial fulfillment of the requirements \\ for the degree of \\ Master of Science \\ Department of Physics \\ Carleton University \\ Ottawa-Carleton Institute of Physics \\ Ottawa, Canada \\ August 31, 2011
}

Copyright (c) 2011 Kathryn A. D. Hally 
Library and Archives

Canada

Published Heritage

Branch

395 Wellington Street Ottawa ON K1A ON4 Canada
Bibliothèque et

Archives Canada

Direction du

Patrimoine de l'édition

395, rue Wellington

Ottawa ON K1A ON4

Canada
Yourfile Votre référence

ISBN: 978-0-494-83201-1

Our file Notre référence

ISBN: 978-0-494-83201-1
NOTICE:

The author has granted a nonexclusive license allowing Library and Archives Canada to reproduce, publish, archive, preserve, conserve, communicate to the public by telecommunication or on the Internet, loan, distribute and sell theses worldwide, for commercial or noncommercial purposes, in microform, paper, electronic and/or any other formats.

The author retains copyright ownership and moral rights in this thesis. Neither the thesis nor substantial extracts from it may be printed or otherwise reproduced without the author's permission.
AVIS:

L'auteur a accordé une licence non exclusive permettant à la Bibliothèque et Archives Canada de reproduire, publier, archiver, sauvegarder, conserver, transmettre au public par télécommunication ou par l'Internet, prêter, distribuer et vendre des thèses partout dans le monde, à des fins commerciales ou autres, sur support microforme, papier, électronique et/ou autres formats.

L'auteur conserve la propriété du droit d'auteur et des droits moraux qui protège cette thèse. $\mathrm{Ni}$ la thèse ni des extraits substantiels de celle-ci ne doivent être imprimés ou autrement reproduits sans son autorisation.
In compliance with the Canadian Privacy Act some supporting forms may have been removed from this thesis.

While these forms may be included in the document page count, their removal does not represent any loss of content from the thesis.
Conformément à la loi canadienne sur la protection de la vie privée, quelques formulaires secondaires ont été enlevés de cette thèse.

Bien que ces formulaires aient inclus dans la pagination, il n'y aura aucun contenu manquant. 


\section{Abstract}

We compute the Higgsstrahlung cross section, $e^{+} e^{--} \rightarrow Z^{0} H$, in an $S O(5) \times U(1)_{B-L}$ gauge-Higgs unification model, the Minimal Composite Higgs Model (MCHM) with the Medina-Shah-Wagner fermion embedding. We observe that the MCHM cross section is strongly suppressed at high energies in comparison with that of the Standard Model due to cancellations between successive Kaluza-Klein coupling contributions. We further investigate the role of particle mixing and decay widths in the cross-section couplings and behaviour. Finally, we determine that several characteristics of the model couplings which lead to this suppression - notably the magnitude and sign of the first KK mode coupling - are observable at an electron-positron collider such as the proposed International or Compact Linear Colliders. 


\section{Acknowledgements}

First and foremost, I would like to thank my supervisor, Heather Logan, who has been an incredible source of knowledge, guidance and support throughout this research project. It has been both a pleasure and an honour to learn from her.

I would like to thank the amazing community of people at Carleton University; my officemates for their advice, Eva Lacelle and Joanne Martin for their endless patience with my questions and troubles, and all of the graduate students for the sanity-checks and good times.

Fmally, thanks to all of my friends and family for their unconditional love and wisdom throughout this process. Special thanks to my amazing parents, who are a continual source of inspiration to me, and to my wonderful fiance Ben, for his infinite patience and encouragement. 


\section{Statement of Originality}

Chapters two and three of this thesis predominantly review material previously presented in the literature, and are referenced accordıngly The new research and analysis that make up the body of this thesis can be found in chapter four This research is entirely new and original (to my knowledge), and has not yet been submitted to publication or as the final project of another degree In the appendices we present further background and suppporting information, notably the Feynman rules of the model, these have been derived independently and cross-checked against the literature where possible, as references indicate 


\section{Contents}

1 Introduction 1

2 The Standard Model of Particle Physics 4

2.1 Introduction to the Standard Model . . . . . . . . . . . . . . . . 4

2.1.1 Forces and particles.................. 4

2.1.2 Structure of the theory ................ 7

2.2 Electroweak Symmetry Breaking . . . . . . . . . . . . . 8

2.2.1 The Higgs Mechanism . . . . . . . . . . . . . 10

2.3 Problems with the Standard Model . . . . . . . . . . . . . . . 15

2.3.1 The Hierarchy Problem . . . . . . . . . . . . . 15

2.3.2 Other problems with the Standard Model . . . . . . . . . . . . 17

3 The Minimal Composite Higgs Model 19

3.1 An introduction to warped-geometry models . . . . . . . . . . 19

3.1 Randall-Sundrum $1 \ldots \ldots . \ldots 19$ 
312 The AdS/CFT correspondence and composite Higgs bosons in RS Models

32 The Minimal Composite Higgs Model

321 Symmetries of the model

322 Model Lagrangians

323 The gauge boson sector 28

324 The fermion sector

325 Electroweak Symmetry Breaking in the MCHM

326 The Coleman-Weinberg potential

41 Higgsstrahlung as a probe of compositeness

42 Two equivalent perspectives

43 A first approximation Higgsstrahlung without particle mixing

432 Cross sections in the Effective Vertex Approach

433 Cross sections in the Effective Cross-section Approach 
441 Comparison and analysis of cross sections

442 Coupling extraction

5 Conclusions

51 Discussion and Conclusions

52 Future Research

$\begin{array}{lll}\text { A } 1 \text { Bessel Functions } & 102\end{array}$

$\begin{array}{ll}\text { A } 2 \text { Meijer G Functions } & 104\end{array}$

A 3 The Generalized Hypergeometric Function 105

$\begin{array}{ll}\text { A } 4 \text { Lommel Functions } & 105\end{array}$

$\begin{array}{ll}\text { A } 5 \text { Struve Functions } & 105\end{array}$

A 6 The Confluent Hypergeometric Functions 105

$\begin{array}{lr}\text { B Generators } & 106\end{array}$

$\begin{array}{lll}\text { B } 1 \text { Definition } & 106\end{array}$

$\begin{array}{ll}\text { B } 2 \text { Commutation relations } & 108\end{array}$

$\begin{array}{lll}\text { B } 3 \text { Expansions of } A_{M N}^{a} & 108\end{array}$

$\begin{array}{ll}\text { C Derivation of Feynman Rules } & 109\end{array}$

$\begin{array}{lll}\text { C } 1 \text { Propagators } & 109\end{array}$

$\begin{array}{ll}\text { C } 11 \text { Gauge Boson } A_{\mu} & 110\end{array}$ 
C 12 Fifth Component $A_{5}^{a}$

C 2 Profiles without particle mixıng

C 21 Boson Profiles

112

C 22 Fermion Profiles

114

C 23 Higgs Profile

C 3 Profiles with particle mixing

C 31 Gauge Bosons

117

C 32 Fermons

C 4 Vertices

C 414 -particle interaction terms $W^{3_{L} R} W^{3_{L} R} H H$

C 42 3-particle interaction Three gauge bosons

135

C 43 3-particle interaction Fermion-fermion-boson

139

C 5 The Relation Between $g$ and $g_{5}$

C 51 From the four gauge boson vertex

C 52 From a $\bar{f} f A_{\mu}$ vertex

143

D Gauge Boson Decay Widths

D 1 Boson decay to formion final states 145

D 2 Gauge boson decay to a Higgs final state 146

$\begin{array}{ll}\text { D } 3 \text { Gauge boson decay to two other gauge bosons } & 147\end{array}$ 
E Analytic integrals for ECA cross-section calculations

E.1 Performing the integrations in the 5D coupling factor $Z_{G}(c, q) \ldots 148$

References 


\section{List of Tables}

21 Forces and their relative strengths 5

22 The particle spectrum of the Standard Model 5

23 Properties of the Standard Model gauge bosons $\quad 7$

31 The gauge boson spectrum of the MCHM 30

32 Profiles associated to fermıon chiralitıes with different BCs 36

33 Coupling terms that lead to boson mixing after EWSB 38

34 Sectors of gauge bosons mixed by EWSB through the Higgs vev $v$ The third group of particles is given in the physical basis because it is in this basis that the boundary conditions are imposed

35 Sectors of quarks mixed by EWSB through the Higgs vev $v$ Recall that $t_{1_{L}}, b_{1_{L}}$ and $\hat{t}_{2_{R}}, B_{3_{R}}^{\prime}$ ale the left- and right-handed SM quarks

36 Sectors of leptons mixed by EWSB through the Higgs vev $v$ Recall that $n_{1_{L}}, \ell_{1_{L}}$ and $\hat{n}_{2_{R}}, L_{3_{R}}^{\prime}$ are the left- and right-handed SM leptons 
3.7 Initial parameters used to solve the Coleman-Weinberg potential, as well as the resulting $k, v, L_{0}$ and $L_{1}$. The parameters with superscript $q_{3}$ refer to those of the third quark generation, while $c_{\text {lght }}$ applies to all other fermions. . . . . . . . . . . . . . . . . . 56

3.8 Table of $Z, X$, and $A^{\hat{3}}$ boson mass both before and after EWSB. . . 56

4.1 Cross-section data used for coupling extraction. . . . . . . . . . . . . 81

4.2 Cross-section data used for coupling extraction. . . . . . . . . . . . . 93

C.1 Possible couplings of $Z, X$ or $A_{\mu}^{\hat{3}}$ bosons to lepton anti-lepton pairs. The $L, R$ subscripts have been suppressed. Refer to Eqs. (3.23-3.28) for the multiplet structure. . . . . . . . . . . . . . 142 


\section{List of Figures}

2.1 Loop diagrams expected to contribute Planck scale contributions to the Higgs mass. Dashed lines represent Higgs, $W$ or $Z$ boson contributions. Solid lines represent fermion contributions; the largest is from the top

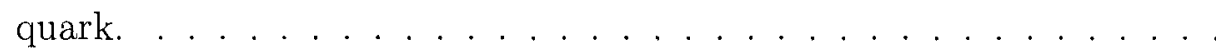

3.1 A schematic representation of the MCHM spacetime. Note that the symmetry group $S U(3)_{c}$ is unbroken everywhere.

3.2 A schematic of the KK mode mass distributions for the $Z, X$ and $A^{\hat{3}}$ bosons both before (solid grey lines) and after (black dotted lines) EWSB. Numeric values for a particular parameter set can be found in Table 3.8 in Sec. 3.2.7. Not to scale. . . . . . . . . . . . . . . . .

3.3 The integrand of the Coleman-Weinberg potential for $k L=30, c_{1}=$ $0.24, c_{2}=-0.41, c_{3}=0.58, M_{1}=2.3$, and $M_{2}=0.4 ;$ these values result in a Higgs vev corresponding to $\theta_{G}\left(L_{1}\right)=0.318 \ldots \ldots$. . . .

3.4 A plot of the relationship between the Higgs vev $v$ and $L_{1}$ for $k L=30$. The dotted lines indicate the ideal values of $v=246 \mathrm{GeV}$ and $L_{1}=$

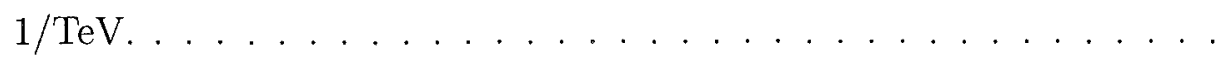


4.1 The Higgsstrahlung interaction, $e^{+} e^{-} \rightarrow Z^{0} H \ldots \ldots \ldots$

4.2 The $e^{+} e^{-} \rightarrow Z^{(0)} H$ from 4D (dotted line) and 5D (solid line) plotted with the SM cross section (dashed line). The units of $\sigma$ and $q$ are $[\mathrm{GeV}]^{-2}$ and $[\mathrm{GeV}]$ respectively. Note that the $4 \mathrm{KK}$ and $6 \mathrm{KK}$ curves are virtually indistinguishable from the full MCHM curve until the energy begins to approach their respective cut-off KK masses. . . . .

4.3 A plot of the $4 \mathrm{D} e^{+} e^{-} \rightarrow Z^{(0)} H$ cross section times $q^{2}$ including an increasing number of KK modes, up to 6 (dotted lines). Also plotted is the cross section obtained from 5D (solid line). The units of $\sigma$ and $q$ are $[\mathrm{GeV}]^{-2}$ and $[\mathrm{GeV}]$ respectively. . . . . . . . . . . .

4.4 The influence of widths on the Higgsstrahlung cross section. The full MCHM does not include widths. Note that these widths do not include KK fermion states. . . . . . . . . . . . . . . .

4.5 The ratio of the squared sum of the left-handed Higgsstrahlung interaction couplings with respect to the left-handed SM Higgsstrahlung coupling plotted versus the number of included KK modes. . . . . . . . . . . . .

4.6 The ratio of the squared sum of the right-handed Higgsstrahlung interaction couplings with respect to the right-handed SM Higgsstrahlung coupling plotted versus the number of included KK modes.

4.7 The squared sum of Higgsstrahlung to SM coupling ratio (both left- and right-handed) plotted on a log-log scale versus the mass of the largest included KK mode. The parameters of the fit are $a=3.7 \pm 0.2, b=-8.8 \pm 0.9$ and $c=-0.40 \pm 0.01 \ldots \ldots \ldots \ldots$ 
48 The squared sum of Higgsstrahlung to SM coupling ratio plotted on a log log scale versus the number of KK modes $n$ plus one The parameters of the fit are $a=-06 \pm 02$ and $b=-550 \pm 007$

49 Extraction of $Z^{(0)}$ and $Z^{(1)}$ couplings from a $\chi^{2}$ at 95 percent confidence using the following data points 05 and $1 \mathrm{TeV}$ (light grey, vertical), 1 and $3 \mathrm{TeV}$ (medium grey, horızontal), 05 and $3 \mathrm{TeV}$ (dark grey, centered), 05,1 , and $3 \mathrm{TeV}$ (black, centered) The coupling ratios are expected to be $C_{Z}^{(0)}=1016, C_{Z}^{(1)}=-1124$

410 Extraction of $Z^{(1)}$ and $Z^{(2)}$ couplings from a $\chi^{2}$ at 95 percent confidence The data points used, from the outside in, are 05 and $3 \mathrm{TeV}$, 1 and $3 \mathrm{TeV}, 05,1$, and $3 \mathrm{TeV}, 05,1,3$ and $5 \mathrm{TeV}$ The coupling ratios are expected to be $C_{Z}^{(1)}=-1124, C_{Z}^{(1)}=0143$

411 The Higgsstrahlung cross section times $q^{2}$ with full mixing but no widths, compared with the approximate cross sections of Fig (42) and the SM

412 A comparison of the square Higgsstrahlung coupling sum - divided by the corresponding SM coupling including particle mixing to the squared sums depicted in figures 45 and 46 of the previous section

413 The Higgsstrahlung cross section times $q^{2}$ with $6 \mathrm{KK}$ modes, widths and full particle mixing compared with the corresponding cross section without mixing, Fig 44 Only boson and SM fermion decay channels are included in the widths 
4.14 The first $Z$ KK resonance peak in the Higgsstrahlung cross section times $q^{2}$. The figure compares cross sections without widths, with only boson widths, and with both boson and fermion widths for $M_{1}=1.5,2.2 .92$

4.15 Extraction of $Z^{(0)}$ and $Z^{(\mathrm{I})}$ couplings from a $\chi^{2}$ at 95 percent confidence level. The data points used, from the outside in, are: 1 and $3 \mathrm{TeV} ; 0.5$ and $3 \mathrm{TeV} ; 0.5,1$, and $3 \mathrm{TeV} ; 0.5,1,3$ and $5 \mathrm{TeV}$. The coupling ratios are expected to be $C_{Z}^{(0)}=0.953, C_{Z}^{(1)}=-1.052$, with $M_{1}=1.5 . \quad . \quad . \quad 94$

4.16 Extraction of $Z^{(1)}$ and $Z^{(2)}$ couplings from a $\chi^{2}$ at 95 percent confidence level. The data points used, from the outside in, are: 0.5 and $3 \mathrm{TeV} ; 1$ and $3 \mathrm{TeV} ; 0.5,1$, and $3 \mathrm{TeV} ; 0.5,1,3$ and $5 \mathrm{TeV}$. The coupling ratios are expected to be $C_{Z}^{(1)}=-1.052, C_{Z}^{(2)}=0.134$, with $M_{1}=1.5 . \ldots$

4.17 Extraction of $Z^{(1)}$ and $Z^{(2)}$ couplings from a $\chi^{2}$ at 95 percent confidence level. The data points used, from the outside in, are: 0.5 and $3 \mathrm{TeV}$; 1 and $3 \mathrm{TeV} ; 0.5,1$, and $3 \mathrm{TeV} ; 0.5,1,3$ and $5 \mathrm{TeV}$. Here we have set the width of $Z^{(2)}$ equal to its mass, with $M_{1}=2.2$. . . . . . . . 


\section{Chapter 1}

\section{Introduction}

In the Standard Model (SM) of partıcle physics, subatomic partıcles acquire mass when they interact with a single elementary particle called the Higgs boson. However, the SM Higgs mechanism has at least two problems First, the flavour puzzle it cannot explain why the masses of leptons and quarks fall into an ordered pattern Second, the hierarchy problem though loop diagrams contribute terms on the Planck scale $\left(M_{p} \approx 10^{19} \mathrm{GeV}\right)$ to the calculation of the Higgs mass, the Higgs mass is required to be on the weak scale $M_{w} \approx 100 \mathrm{GeV}$ Elmminating the Planck scale terms in the SM requires an undesirable and extreme fine-tuning of the model parameters

Both problems can be solved by constructing a quantum field theory in a warped five-dimensional (5D) Ant1-de-Sitter (AdS) spacetıme bounded by two fourdimensional (4D) boundaries, an idea first proposed by Randall and Sundrum in 1999 [1] The curvature of the fifth dimension causes the energy scale to decrease exponentially such that the first boundary has energies up to the Planck scale while the second has energies only up to the weak scale The hierarchy problem can be solved by placing the Higgs boson near the weak-scale boundary, causing any large 
mass contributions to be 'warped down' to the weak scale. The flavour puzzle can also be explained by the locations of the leptons and quarks in the fifth dimension, as particles close to the Higgs boson will have large masses while those farther away will have smaller masses.

According to the AdS/CFT conjecture [2], Randall-Sundrum (RS) models can be identified with a four dimensional strongly coupled conformal field theory $\left(\mathrm{CFT}_{4}\right)$; integrating the 5D theory over the fifth dimension provides an effective picture of this CFT. In this effective 4D perspective, the theory contains an infinite tower of 4D excitations - known as Kaluza-Klein (KK) modes - for each 5D particle; the zero modes of these towers correspond to Standard Model particles. Furthermore, as it is a $5 \mathrm{D}$ theory each four dimensional gauge boson must also have an associated scalar fifth component. As such, RS models have a much larger particle spectrum than the Standard Model. However, the masses of the higher order KK modes are constrained to be above a mass scale $M_{k k}$ defined by the length of the fifth dimension, which must be larger than $1 \mathrm{TeV}$. Therefore the new Large Hadron Collider at CERN will be the first accelerator with the energy reach to observe the proposed extra particles.

A promising class of Randall-Sundrum models are those which employ GaugeHiggs Unification (GHU), where the Higgs boson arises naturally as the scalar fifth component of one the gauge bosons. These models are dual to a $4 \mathrm{D}$ CFT that includes a composite ${ }^{1}$ Higgs boson. The goal of this thesis is to gain a better theoretical understanding of how this composite Higgs interacts with other particles, how its fi-

\footnotetext{
${ }^{1}$ It should be emphasized that for the purposes of this work the term 'composite' is meant to imply that a particle is not point-like, but has some finite size which influences its interactions with other particles. We do not propose to directly probe the constituents of the Higgs, or to comment upon the nature of its internal structure. Indeed, the effective Lagrangian of the 5D theory does not allow for that kind of analysis.
} 
nite size would affect scattering processes, and whether such characteristics could be identifable signatures of the model at experimental colliders. To this end, we present a calculation of the Higgsstrahlung, $e^{+} e^{-} \rightarrow Z^{0} H$, interaction in the Minimal Composite Higgs Model (MCHM) with the fermion representation proposed by Medina, Shah and Wagner (MSW) [3, 4]. This interaction is particularly suitable for our purposes as it is an s-channel interaction, ensuring that the initial interaction energy is transferred directly into the Higgs coupling. As the structure of this Higgs interaction is similar to that of the loop interactions that yield Planck scale mass corrections in the SM, we expect that the MCHM Higgsstrahlung cross section will demonstrate a similar suppression when compared with the SM cross section. The resulting rapid decay in the cross section with increasing collision energy would be characteristic of a composite particle form factor.

The structure of the thesis is as follows. In Chapter 2 we review the Standard Model, electroweak symmetry breaking (EWSB), and the problems that have led to investigation beyond the SM. In Chapter 3, we briefly introduce Randall-Sundrum models before providing a detailed description of the Minimal Composite Higgs model structure and the Medina-Shah-Wagner fermion representation. Chapter 4 provides the calculations, results and discussion of the MCHM Higgsstrahlung cross section and relevant coupling behaviour. We further provide a brief analysis of the feasibility of coupling extraction at both the International Linear Collider (ILC) and the Compact LInear Collider (CLIC). Although the most relevant formulas are provided in the text, detailed derivations of the model Feynman rules can be found in Appendix C. 


\section{Chapter 2}

\section{The Standard Model of Particle Physics}

\subsection{Introduction to the Standard Model}

\subsubsection{Forces and particles}

The Standard Model (SM) of particle physics is the most complete theory to date that describes the fundamental interactions and behaviour of subatomic particles [5]. It contains three forces: the strong force, the weak force and the electromagnetic force.

The Standard Model does not include gravity. Gravity is an extremely weak force; even the Weak force itself is $10^{25}$ times stronger (see Table 2.1). As a result, gravity is negligible at the relevant scales for particle interactions, and may safely be neglected. Furthermore, gravity is described by General Relativity (GR), while the 
Table 21 Forces and their relative strengths

\begin{tabular}{|c|c|c|}
\hline Interaction & Strength & Range \\
\hline \hline Strong & 1 & $10^{-15} \mathrm{~m}$ \\
\hline Electromagnetism & $10^{-2}$ & $\infty$ \\
\hline Weak & $10^{-5}$ & $10^{-18} \mathrm{~m}$ \\
\hline Gravity & $10^{-39}$ & $\infty$ \\
\hline
\end{tabular}

Standard Model is a quantum field theory (QFT), and physicists have yet to successfully express GR in an effectıve QFT formalısm Until such a theory of quantum gravity is developed, unifyıng the two remains problematıc

Table 22 The particle spectrum of the Standard Model

\begin{tabular}{|l|c|c|c||c|c|}
\hline \multicolumn{4}{|c||}{ Fermions } & \multicolumn{2}{c|}{ Bosons } \\
\hline \hline Generation & 1 & 2 & 3 & Gauge & Scalar \\
\hline \multirow{3}{*}{ Leptons } & $\nu_{e}$ neutrino & $\nu_{\mu}$ neutrino & $\nu_{\tau}$ neutrino & $Z$ & \\
& Electron, $e^{-}$ & Muon, $\mu$ & Tau, $\tau$ & $W^{ \pm}$ & \multirow{2}{*}{ Higgs, $H$} \\
\hline \multirow{3}{*}{ Quarks } & Up, $u$ & Charm, $c$ & Top, $t$ & Photon, $\gamma$ & \\
& Down, $d$ & Strange, $s$ & Bottom, $b$ & Gluon, $g$ & \\
\hline
\end{tabular}

The particle spectrum of the Standard Model is outlined in Table 22 It contains twelve fundamental fermions - or spin $1 / 2$ particles - as well as their ant1particles six leptons (the electron, muon, tau and their associated neutrinos) and six 
quarks (up, down, strange, charm, top and bottom). Unlike the leptons, the quarks are never found individually; they combine in pairs and triplets to form composite hadrons, such as the proton and neutron.

The SM also contains several vector (spin 1) gauge bosons which carry the three fundamental forces. The properties of these bosons are summarized in Table 2.3. The photon is a neutral, massless boson that carries the electromagnetic force, and therefore couples only to charged particles.

The gluons are also massless bosons, but they carry the strong force. There are eight different types of gluons, identified by combinations of the three strong charges, or colours. Gluons only couple to each other and to quarks, as they are the only other particles which carry colour charge.

The $W^{ \pm 1}$ and $\mathrm{Z}$ are massive bosons that carry the weak force. The $W^{ \pm}$have charge \pm 1 and have masses of $80.398 \pm 0.023 \mathrm{GeV}$ while the $\mathrm{Z}$ boson is neutral and has a mass of $91.1876 \pm 0.0021 \mathrm{GeV}$ [6]. They interact with each other, the fermions and the Higgs, and the charged $W^{ \pm}$additionally couple to the photon.

Finally, the Standard Model also includes a single scalar - spin 0 - boson, known as the Higgs. It is through their couplings to the Higgs that particles acquire their masses; therefore the Higgs couples only to massive particles at tree level. ${ }^{2}$

\footnotetext{
${ }^{1}$ The $W^{+}$and $W^{-}$have opposite charge but the same spin and mass; they are each other's anti-particles.

${ }^{2}$ 'Tree level' indicates calculations up to zeroth order in perturbation theory; such calculations include only Feynman diagrams without loops (which sometimes look like trees).
} 
Table 2.3: Properties of the Standard Model gauge bosons.

\begin{tabular}{|c|c|c|c|c|}
\hline Interaction & Gauge Boson & Mass & Spin & Acts On \\
\hline \hline Strong & Gluon & 0 & 1 & Colour charge \\
\hline Electromagnetism & Photon & 0 & 1 & Electric Charges \\
\hline Weak & $W^{ \pm}, Z^{0}$ & $80,91 \mathrm{GeV}$ & 1 & Weak Charge \\
\hline
\end{tabular}

\subsubsection{Structure of the theory}

The Standard Model is a non-abelian gauge theory as well as an effective quantum field theory. As a quantum field theory, the Standard Model describes the subatomic world in terms of fields. These fields are quantized, and their quanta correspond to the elementary particles. The theory is an effective field theory (EFT) in that it is accurate only up to some cut-off energy scale, and describes only the physics that is relevant over this energy range. Above the cut-off scale the SM is expected to break down due to the emergence of physics that is not included within the theory. It is possible that the SM is valid all the way up to the Planck scale $\left(M_{p} \approx 10^{19}\right.$ $\mathrm{GeV}$ ), where gravity becomes relevant. However, there are many compelling reasons to believe that the SM cutoff is in fact much lower $(\approx 1 \mathrm{TeV})$, and that some form of new physics - as yet undetected -- manifests at this scale; this concept will be discussed in further detail in Sec. 2.3.

As a gauge theory, the Standard Model Lagrangian is invariant under a certain symmetry group, $S U(3)_{c} \times S U(2)_{L} \times U(1)_{Y}$ [5]. For each generator of the group there is a corresponding gauge field, the quanta of which are the forcc-carr ying gauge 
bosons. It is non-abelian because the generators of the $S U(3)$ and $S U(2)$ sectors are non-commutative.

The $S U(3)$ symmetry describes the strong force sector, and its 8 generators correspond to the 8 types of gluons. This sector is described by an EFT within the SM known as Quantum Chromodynamics (QCD) [7].

The electromagnetic force is described by a $U(1)_{E M}$ EFT known as Quantum Electrodynamics (QED) [8]. The single generator of the abelian - or commutative $U(1)$ symmetry group corresponds to the photon. However, in the Standard Model the electromagnetic sector is unified with the weak sector; the resulting electroweak sector is described by the $S U(2)_{L} \times U(1)_{Y}$ symmetry group which contains the $U(1)_{E M}$ group of QED. The three generators of $S U(2)_{L}$ along with the single generator of $U(1)_{Y}$ correspond to four gauge fields that mix to create the photon, $W_{\mu}^{ \pm}$and $\mathrm{Z}$ bosons.

\subsection{Electroweak Symmetry Breaking}

As seen in the preceding section, the gauge symmetry group of the Standard Model plays an important role in its structure. Such symmetries are very important in physics, as according to Noether's theorem,

In any theory described by a Lagrangian, a symmetry of the Lagrangian corresponds to a conservation law [9]. 
As a gauge theory, the SM must obey gauge invariance, this requires that the Lagrangian remains invariant under local ${ }^{3}$ gauge transformations, which have the form

$$
\begin{gathered}
A_{\mu} \rightarrow U(x) A_{\mu} U^{\dagger}(x)+\frac{\imath}{g} U(x) \partial_{\mu} U^{\dagger}(x) \\
\phi \rightarrow U(x) \phi
\end{gathered}
$$

for gauge fields $A_{\mu}(\operatorname{spm} 1)$ and matter fields $\phi\left(\operatorname{spin} 0\right.$ or $\left.\pm \frac{1}{2}\right)$ respectively [10] Here $U(x)$ is an element of the symmetry group of the Lagrangian, and $g$ is the gauge coupling

However, a mass term for such a gauge boson does not obey this invarlance For example, consider the simplest case, a local $U(1)$ symmetry where $U(x)=\exp [\imath g \eta(x)]$ Then

$$
\frac{1}{2} M^{2} A_{\mu} A^{\mu} \rightarrow \frac{1}{2} M^{2}\left(A_{\mu} A^{\mu}+2 \partial_{\mu} \eta(x) A^{\mu}+\partial_{\mu} \eta(x) \partial^{\mu} \eta(x)\right)
$$

Therefore for the gauge symmetry to be preserved the associated gauge bosons must be massless

This presents a problem, for, as previously discussed, the weak bosons $W^{ \pm}$ and $Z$ are not massless, but have well-measured masses of about 80 and $91 \mathrm{GeV}$ respectively [6] As such, there must cxist some mechanısm by which the electroweak symmetry is spontaneously ${ }^{4}$ broken such that the weak gauge bosons are given masses but the photon remains massless We therefore require the following symmetrybreaking scheme

$$
S U(2)_{L} \times U(1)_{Y} \rightarrow U(1)_{E M},
$$

\footnotetext{
${ }^{3}$ If $U$ is a constant, it is a global gauge transformation, a coordinate-dependent $U(x)$ results in a local gauge transformation

4 'Spontaneous symmetry breaking' means that the theory is defined so as to obey the symmetry, but the system it describes is always observed in a particular non-symmetric state
} 
where only the electromagnetic symmetry $U(1)_{E M}$ remains intact.

Such a mechanism must also introduce three new massless bosons - known as Goldstone bosons - to the theory according to Goldstone's theorem,

"For every spontaneously broken continuous symmetry, the theory must contain a massless particle." $[11,12]$

However, one can always perform a gauge transformation to work in a particular gauge - known as unitary gauge - where the three extra degrees of freedom represented by the three Goldstone bosons become the longitudinal polarizations of the $W^{ \pm}$and $Z$ bosons [10]. In essence, the process of electroweak symmetry breaking transforms a massless gauge boson with two polarizations into a massive boson with three polarizations.

\subsubsection{The Higgs Mechanism}

One method of breaking the electroweak symmetry is by use of the Higgs mechanism, which was first proposed by Peter Higgs in 1964 [13, 14]. The Higgs mechanism, in its original formulation, introduces a complex scalar doublet

$$
\Phi=\left(\begin{array}{c}
\phi^{+} \\
\phi^{0}
\end{array}\right)=\frac{1}{\sqrt{2}}\left(\begin{array}{c}
\phi_{1}+i \phi_{2} \\
\phi_{3}+i \phi_{4}
\end{array}\right)
$$

into the SM Lagrangian, where $\phi^{+}$is charged, $\phi^{0}$ is neutral, and $\phi_{1}, \phi_{2}, \phi_{3}, \phi_{4}$ denote real scalar degrees of freedom. The bosonic part of the electroweak Lagrangian then takes the form

$$
L_{B}=-\frac{1}{4} W_{\mu \nu}^{a} W^{a \mu \nu}-\frac{1}{4} B_{\mu \nu} B^{\mu \nu}+\left|D_{\mu} \Phi\right|^{2}-V(\Phi)
$$


where $V(\Phi)$ is a potentral energy term that describes the self-interactions of $\Phi$, we use the metric convention $\eta_{\mu \nu}=\operatorname{diag}(1,-1,-1,-1)$, and the covariant derivitive $D_{\mu}$ is given by

$$
D_{\mu}=\partial_{\mu}-\imath \frac{g}{2} \sigma^{a} W_{\mu}^{a}-\imath \frac{g^{\prime}}{2} Y B_{\mu}
$$

In the above $W_{\mu}^{a}(a=1,2,3)$ denotes the three bosons associated with the $\mathrm{SU}(2)_{L}$ symmetry, $B_{\mu}$ is the single boson of the $\mathrm{U}(1)_{Y}$ symmetry, $\sigma^{a}$ are the Paul matrices, $g$ and $g^{\prime}$ are coupling constants and $Y$ is the hypercharge

The most general renormalizable and $\mathrm{SU}(2)_{L}$ invariant potential $V(\Phi)$ for the scalar doublet is given by

$$
V(\Phi)=\mu^{2}|\Phi|^{2}+\lambda|\Phi|^{4}
$$

where $\lambda>0$ and $|\Phi|^{2}=\Phi^{\dagger} \Phi$ If we require that $\mu^{2}<0$, the potential will have a minimum value at

$$
\left\langle\Phi^{\dagger} \Phi\right\rangle=\frac{-\mu^{2}}{2 \lambda}=\frac{v^{2}}{2}
$$

As this defines only the expectation value of $\Phi^{\dagger} \Phi$, we may choose the expectation value of $\Phi$ to be

$$
\langle\Phi\rangle=\frac{1}{\sqrt{2}}\left(\begin{array}{c}
0 \\
v
\end{array}\right)
$$

In making this choice we have effectively chosen $\phi_{3}$ to be a real scalar field with vacuum expectation value (vev) $v$, while $\phi_{1}, \phi_{2}$, and $\phi_{4}$ are the massless Goldstone bosons which disappear from the partıcle spectrum to become the longitudinal polarızations of our massive bosons We may therefore write the full scalar doublet in the unitary 
gauge as

$$
\Phi=\frac{1}{\sqrt{2}}\left(\begin{array}{c}
0 \\
H(x)+v
\end{array}\right)
$$

where $H(x)$ is a scalar field callod the Higgs boson The teims involving $W_{\mu}$ and $B_{\mu}$ in the covariant derıvative result in the following interaction terms between the scalar and the vector bosons,

$$
\begin{aligned}
\left|D_{\mu} \Phi\right|^{2} & =\left|\partial_{\mu} \Phi-\imath \frac{g}{2} \sigma^{a} W_{\mu}^{a} \Phi-\imath \frac{g^{\prime}}{2} Y B_{\mu} \Phi\right|^{2} \\
& =\left|\frac{1}{\sqrt{2}}\left(\begin{array}{c}
0 \\
\partial_{\mu} H
\end{array}\right)-\frac{\imath(v+H)}{2 \sqrt{2}}\left(\begin{array}{c}
g\left(W_{\mu}^{1}-\imath W_{\mu}^{2}\right) \\
-g W_{\mu}^{3}+g^{\prime} B_{\mu}
\end{array}\right)\right|^{2} \\
& =\frac{1}{2} \partial_{\mu} h \partial^{\mu} H+\frac{1}{8}(v+H)^{2}\left[g^{2}\left|W_{\mu}^{1}-\imath W_{\mu}^{2}\right|^{2}+\left(-g W_{\mu}^{3}+g^{\prime} B_{\mu}\right)^{2}\right]
\end{aligned}
$$

where we have used the fact that $Y=1$ for the Higgs doublet $\Psi$, and $H=H(x)$ In $\mathrm{Eq}(212)$ we can see that when $(v+H)^{2}$ is expanded the terms involving $v^{2}$ will produce mass terms for the gauge fields However, the gauge fields we observe in nature are not in fact the gauge fields $W_{\mu}^{1}, W_{\mu}^{2}, W_{\mu}^{3}$ and $B_{\mu}$ We observe instead the $W$ bosons, $Z$ boson and the photon $\left(V_{\mu}\right),{ }^{5}$ which are linear combinations of our orıginal fields

$$
\begin{aligned}
W_{\mu}^{ \pm} & =\frac{1}{\sqrt{2}}\left(W_{\mu}^{1} \mp \imath W_{\mu}^{2}\right) \\
Z_{\mu} & =\cos \theta_{w} W_{\mu}^{3}-\sin \theta_{w} B_{\mu} \\
V_{\mu} & =\sin \theta_{w} W_{\mu}^{3}+\cos \theta_{w} B_{\mu}
\end{aligned}
$$

\footnotetext{
${ }^{5}$ Here we represent the photon by $V_{\mu}$ to avoid confusion with $A_{\mu}^{a}$, which will be used to generically represent gauge bosons in the next chapter
} 
where

$$
\cos \theta_{w}=\frac{g}{\sqrt{g^{2}+g^{\prime 2}}} \quad \text { and } \quad \sin \theta_{w}=\frac{g^{\prime}}{\sqrt{g^{2}+g^{\prime 2}}} .
$$

The Weinberg angle $\theta_{w}$ has been experimentally determined to be $\theta_{w}=28.75$ degrees [6]. Note that the coupling constants $g$ and $g^{\prime}$ must be related to the electromagnetic constant $e$ through $e=g \sin \theta_{w}=g^{\prime} \cos \theta_{w}$ for the photon to have the proper coupling strength.

In terms of these superpositions, Eq. (2.12) becomes

$$
\begin{aligned}
\left|D_{\mu} \Phi\right|^{2}= & \frac{1}{2} \partial_{\mu} H \partial^{\mu} H+\frac{1}{8}\left(v^{2}+2 v H+H^{2}\right) \\
& \times\left[2 g^{2} W_{\mu}^{+} W^{\mu-}+\left(g^{2}+g^{2}\right) Z_{\mu} Z^{\mu}\right] .
\end{aligned}
$$

The masses of the bosons may be read off the Lagrangian as their squares are merely the mass term couplings; we therefore obtain

$$
M_{W}^{2}=\frac{g^{2} v^{2}}{4}, \quad M_{z}^{2}=\frac{v^{2}}{4}\left(g^{2}+g^{2}\right)=\frac{g^{2} v^{2}}{4 \cos ^{2} \theta_{w}}, \quad M_{A}^{2}=0 .
$$

Therefore the $W_{\mu}^{ \pm}$and $Z_{\mu}$ bosons gain mass terms due to the non-zero Higgs vev $v$. The photon, however, does not couple to the Higgs; therefore it remains massless.

We can also read off the couplings of $W$ and $Z$ bosons to the Higgs field $H{ }^{6}$

$$
G_{\mu} G_{\nu} H H \rightarrow \frac{\imath g_{G}^{2}}{2} \eta_{\mu \nu}, \quad G_{\mu} G_{\nu} H \rightarrow \frac{\imath g_{G}^{2} v}{2} \eta_{\mu \nu}
$$

where $G=Z, W^{ \pm}$and $g_{W}=g, g_{Z}=g \sec \theta_{w}$.

The Higgs itself also has a mass, which can be read off the first term of the Higgs potential

$$
V(\Phi)=\frac{\mu^{2}}{2}(v+H)^{2}+\frac{\lambda}{4}(v+H)^{4}=-\mu^{2} H^{2}+\lambda v H^{3}+\frac{\lambda}{4} H^{4} .
$$

\footnotetext{
${ }^{6}$ Recall that when reading couplings from Lagrangian terms we must always multiply by $\imath$ and a symmetry factor to account for all possible permutations of identical fields, for example, if we have a term $c Z^{\mu} Z_{\mu}$ the coupling is $2 \imath c$ as the two $Z_{\mu}$ fields are interchangeable
} 
along with two other couplings. Therefore we have

$$
M_{H}^{2}=-2 \mu^{2}=2 v^{2} \lambda, \quad H H H \rightarrow 6 \lambda v, \quad H H H H \rightarrow 6 \lambda
$$

Finally, through $W$ boson interactions with muons the Higgs vacuum expectation value $v$ may be related to the Fermi constant $G_{F}=1.16639 \times 10^{-5} \mathrm{GeV}^{-2}$ and evaluated, ${ }^{7}$

$$
v=\left(\sqrt{2} G_{F}\right)^{-\frac{1}{2}}=\left(\frac{g^{2}}{4 M_{W}^{2}}\right)^{-\frac{1}{2}}=246 \mathrm{GeV}
$$

Note that the Higgs mass cannot be determined directly from known experimental quantities as it depends on $\lambda$; however, it can replace $\lambda$ as a parameter of the theory (while $\mu$ can be replaced by known quantity $v$ ). The Higgs mass is constrained from experimental tests to be within the range $114 \mathrm{GeV} \leq M_{H} \lesssim 185 \mathrm{GeV}$. The lower bound of $114 \mathrm{GeV}$ was obtained from direct experimental searches at the Large Electron-Positron Collider (LEP) [16], and is a general bound which applies to most models. However, the upper bound originates from electroweak precision fits to the SM; it is a model-dependent bound that does not necessarily apply to models beyond the SM [17]. Furthermore, results from the Tevatron collider forbid a SM Higgs of mass $160-170 \mathrm{GeV}[18,19]$.

The Higgs mechanism can also give fermions mass in a similar manner through Yukawa terms [11]. A Yukawa term describes the coupling of a Dirac fermion $(\Psi)$ and anti-fermion to a scalar. By adding Yukawa terms involving the Higgs to the model Lagrangian and breaking the electroweak symmetry, we obtain

$$
L_{\mathrm{yuk}}=g_{\mathrm{yuk}} \bar{\Psi} H \Psi \rightarrow g_{\mathrm{yuk}} \bar{\Psi}(H+v) \Psi=g_{\mathrm{yuk}} v \bar{\Psi} \Psi+g_{\mathrm{yuk}} \bar{\Psi} H \Psi
$$

\footnotetext{
${ }^{7}$ The Fermi Constant $G_{F}$ is the coupling strength of the Fermi interaction, a four-fermion interaction proposed by Enrico Fermi in 1933 as part of his description of beta-decay [15].
} 
where the first term on the right-hand side is a fermion mass term. We can see that with an appropriate choice of the Yukawa coupling constant $g_{\text {yuk }}$ we can reproduce the appropriate fermion masses.

\subsection{Problems with the Standard Model}

Although the SM has prevailed through over 40 years of rigorous testing, it still suffers from several deficiencies. The most important of these is the Hierarchy Problem.

\subsubsection{The Hierarchy Problem}

The Standard Model is an incredibly successful theory. It has stood up to over four decades of rigorous experimental tests. However, there are still several questions and problems that it cannot successfully explain. One of the most prominent of these failures is known as the Hierarchy Problem. The Hierarchy Problem is essentially the question of why gravity is so weak; the gravitational force only becomes relevant at mass scales on the order of the Planck mass, $M_{P} \approx 10^{19} \mathrm{GeV}$, while the other forces operate at much lower scales. The Standard Model does not include gravitational effects, and it fails to provide an explanation for why there is such a prominent difference - or hierarchy - in the strength of the gravitational force when compared with the strong and electroweak forces.

In terms of Higgs physics, this problem is equivalent to asking why the Higgs mass parameter $\mu^{2}$ is so small; this parameter sets the scale for the Higgs vev $v$, and by extension all of the particle masses, including that of the Higgs. Radiative corrections from loop diagrams such as those in Fig. 2.1 are expected to add quadratic 
Planck scale corrections to $\mu^{2}$, causing it to become extremely large But the particle masses are known to be much smaller on the weak scale - by experment, therefore any model that employs a Higgs mechanism must provide some way of protecting $\mu^{2}$ from such large corrections In the Standard Model there is no explicit mechanism or
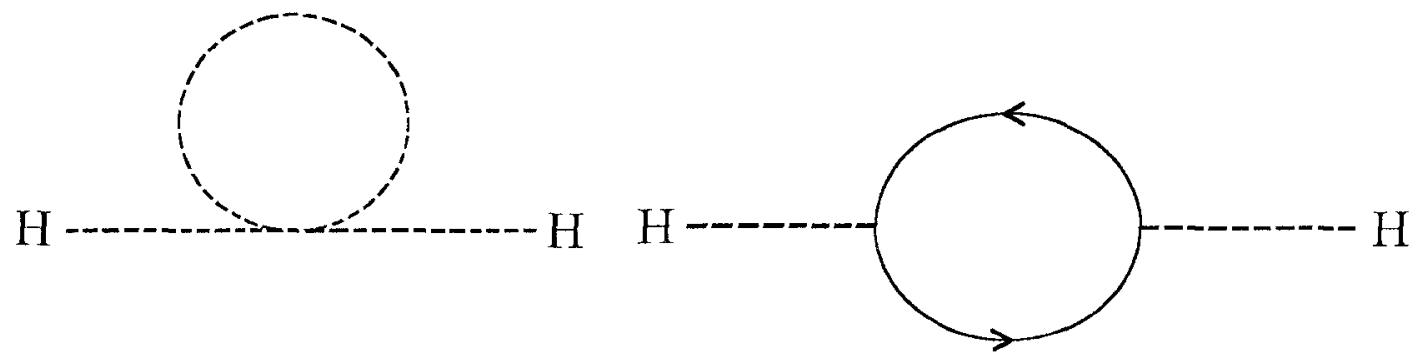

Figure 21 Loop diagrams expected to contribute Planck scale contributions to the Higgs mass Dashed lines represent Higgs, $W$ or $Z$ boson contributions Solid lines represent fermion contributions, the largest is from the top quark

symmetry to protect the Higgs mass As such, there must be an extreme fine-tuning of the model parameters for the square of the Higgs mass to end up on the electroweak scale, roughly 34 orders of magnitude smaller than the model would naturally predict it to be As most physicists consider this sort of fine-tuning to be undesirable and unnatural, it is believed to indicate that the Standard Model is not complete, there must be some further mechanism that manifests above the scale of the Higgs mass and which leads to the suppression or cancellation of the quadratic divergences These new effects must appear around or bolow the TeV scalc for $\mu^{2} \simeq(100 \mathrm{GeV})^{2}$ to be considered natural

There have been many proposals as to what that mechanism might be The 
most popular alternatıve is Supersymmetry [20, 21], a theory in which every Standard Model particle has an associated superpartner The superpartners must be heavier than the SM particles - or we would have identıfied them experımentally by now and the spin of each superpartner differs from that of the corresponding SM particle by $1 / 2$ In this scenarıo, the quadratic corrections to the Higgs mass from each SM particle are cancelled by those fiom their superpartner

Another proposal is that of Little Higgs models [22], where the Higgs is a pseudo-Goldstone boson, it is the Goldstone boson of a spontaneously broken symmetry, but an additional explicit symmetry breakıng gives it a small mass In these cases, the Higgs mass is protected from large quadratic corrections by the symmetries of the model There are many further possibilities as well, including models of composite Higgs bosons [23, 24, 25, 26], and models such as Technicolour, which achieve EWSB without a Higgs $[27,28,29,30,31]$ However, each of these models has their own draw-backs and many still retain hierarchy problems or fine-tuning, though to a lesser degree than the Standard Model

\subsubsection{Other problems with the Standard Model}

Although the Hierarchy problem is the most common incentive and inspiration for proposals of Beyond the Standard Model (BSM) mechanısms, there are several other questions that the Standard Model falls to answer, and which BSM models try to account for

One of these is the large range of different fermion masses, for example, the electron has a mass of $m_{e}=0510998910 \pm 0000000013 \mathrm{MeV}$, whlle the top quark's mass, $m_{t}=172 \pm 2 \mathrm{GeV}$ [6], 1s larger by five orders of magnitude Although the 
SM can describe these masses with Yukawa terms, it cannot explain why the fermion masses are so different. This problem became even more pertinent with the relatively recent discovery that neutrinos also have very tiny non-zero masses [32]. This finding added several orders of magnitude to the range of fermion masses and created a whole new set of problems as the Standard Model does not account for non-zero neutrino masses at all.

A second significant problem is recent accumulation of experimental evidence for Dark Matter [33, 34]. Experimental measurements of the masses of galaxy-clusters have suggested that the universe contains a great deal more matter than can be accounted for by the Standard Model. It is estimated that Standard Model particles make up only 4.6 percent of the universe's mass and energy; roughly 23 percent is the extra Dark Matter, and the remaining percentage is Dark Energy. The primary characteristics of Dark Matter are that it must be massive, and electrically neutral as it does not emit radiation. The are several possibilities for what Dark Matter might be; however, the most common theory currently hypothesizes that it is some sort of Weakly Interacting Massive Particle (WIMP) [34]. Although the existence of Dark Matter has not yet been conclusively confirmed, the body of evidence is growing [35], and many BSM models attempt to include one or more particles which could be a WIMP or some other Dark Matter candidate. 


\section{Chapter 3}

\section{The Minimal Composite Higgs Model}

\subsection{An introduction to warped-geometry models}

Five dimensional (5D) warped geometry models are a class of models based on the Randall-Sundrum (RS) model

\subsubsection{Randall-Sundrum 1}

The first Randall-Sundrum model (RS1) was proposed by Lisa Randall and Raman Sundrum in 1999 as a way of resolving the hierarchy problem [1]

This model is defined in a 5D Ant1-de-Sitter $\left(\mathrm{AdS}_{5}\right)$ space $\mathrm{An} \mathrm{AdS}_{n}$ space is a maximally symmetric spacetıme with a negative curvature The fifth dimension of this spacetime - which we denote by $y$ - is compactified on a circle, such that $y=y+2 \pi r_{c}$ Furthermore, the top and bottom halves of the circle are identified 
with each other, $y=-y$, such that there are two boundaries, one at $y=0$ and one at $y=L=\pi r_{c}$ This is formally known as the $S^{1} / Z_{2}$ orbifold, where $S^{1}$ refers to the circle and $Z_{2}$ to the transformation $y \rightarrow-y$

The metric of this spacetıme is obtained by solving Einstein's equations and is found to be

$$
d s^{2}=e^{-2 k r_{c}|\phi|} \eta_{\mu \nu} d x^{\mu} d x^{\nu}-r_{c}^{2} d \phi^{2}=e^{-2 k|y|} \eta_{\mu \nu} d x^{\mu} d x^{\nu}-d y^{2}
$$

where $\eta_{\mu \nu}=\operatorname{diag}(1,-1,-1,-1), k$ is on the order of the Planck scale, and $y=$ $r_{\mathrm{c}} \phi[1,36,37]$

Energy scales on the boundary at $y=0$ can be as high as the Planck scale, thus this boundary is known as the Planck - or ultraviolet (UV) - brane However, due to the warped nature of the spacetime itself, the energy scale of the boundary at $y=L$ is much smaller, energies are warped down to the $\mathrm{TeV}$ scale, and so this is known as the TeV - or Weak, or infrared (IR) brane As such, through the warped spacetime the model can very naturally explain large hierarchies such as that of the Hierarchy problem

To show this explicitly, consider the Einstein-Hilbert actıon $[1,36]$,

$$
\begin{aligned}
S & =2 M^{3} \int d^{4} x \int_{-L}^{L} d y \sqrt{-\bar{g}_{5}} R_{5}=2 M^{3} \int d^{4} x \sqrt{-\bar{g}_{4}} R_{4} \int_{-L}^{L} d y e^{-2 k|y|} \\
& =\frac{M^{3}}{k}\left[1-e^{-2 k L}\right] \int d^{4} x \sqrt{-\bar{g}_{4}} R_{4}
\end{aligned}
$$

where $M$ is the $5 \mathrm{D}$ mass scale, $R_{5}$ is the $5 \mathrm{D}$ Ricci scalar, $R_{4}$ is the $4 \mathrm{D}$ Ricci scalar, $\bar{g}_{5}$ 1s the determinant of the $5 \mathrm{D}$ metric, and $\bar{g}_{4}$ is the determinant of $\eta_{\mu \nu}$ Comparing this to the usual 4D Einstem-Hilbert action, we find that

$$
M_{p}^{2}=\frac{M^{3}}{k}\left[1-e^{-2 k L}\right]
$$


Given that $k$ is on the order of the Planck mass, we can see that $M \approx M_{p}$; the mass scale relevant for gravitational interactions is not significantly altered by the warped spacetime.

However, consider the action relevant to a Higgs boson that is constrained to the TeV boundary

$$
S=\int d^{4} x \sqrt{-\bar{g}_{4}} e^{-4 k L}\left[\eta^{\mu \nu} e^{2 k L}\left(D_{\mu} \Phi\right)^{\dagger} D_{\nu} \Phi-\lambda\left(\Phi^{\dagger} \Phi-v_{0}^{2}\right)^{2}\right]
$$

where $v_{0}$ is the Higgs vacuum expectation value. Rescaling the Higgs scalar field, $\Phi \rightarrow e^{k L} \Phi$, we find

$$
S=\int d^{4} x \sqrt{-\bar{g}_{4}}\left[\eta^{\mu \nu}\left(D_{\mu} \Phi\right)^{\dagger} D_{\nu} \Phi-\lambda\left(\Phi^{\dagger} \Phi-v_{0}^{2} e^{-2 k L}\right)^{2}\right] .
$$

This is the usual Higgs action, except the vev has become exponentially suppressed, $v=e^{-k L} v_{0}$. Therefore, we can see that the gravitational scale remains the same, but the Higgs vev - and by extension contributions to the Higgs mass - will be suppressed, creating a large hierarchy.

The warped $5 \mathrm{D}$ spacetime also has consequences with respect to the particle spectrum. Each particle corresponds to a five dimensional field. Due to the periodicity of the fifth dimension, these 5D fields can be expressed as a Kaluza-Klein decomposition $[38,39,40]$

$$
A(x, z)=\sum_{n}^{\infty} f\left(m_{n}, z\right) A\left(m_{n}, x\right)
$$

Here $A(x, z)$ denotes the full 5D field. $A\left(m_{n}, x\right)$ is a four dimensional component of $A(x, z)$ known as a Kaluza-Klein mode, which has mass $m_{n}$, and $f\left(m_{n}, z\right)$ is a profile that describes the KK mode's location in the fifth dimension. As a result, from a $4 \mathrm{D}$ effective perspective each 5D particle corresponds to an infinite tower of $4 \mathrm{D} \mathrm{KK}$ modes. The zero modes of such towers correspond to Standard Model particles. 
In the original RS1 model, all of the SM particles were confined to the TeV brane However, a second formulation RS2 - set $L \rightarrow \infty$ such that there was no TeV brane, and placed the particles on the Planck brane [41] Subsequent models have placed the gauge bosons and fermons in the bulk of the fifth dimension $[42,43,44]$, however, it is most common to keep the Higgs elther on or very near to the $\mathrm{TeV}$ brane so as to maintam the niass scale hierarchy Models further differ from each other by cmploying different gauge or global symmetry groups both in the bulk of the fifth dimension and on its boundaries, by including boundary-localized terms in the Lagrangian, and by considering a fifth dimension with a different or infinite length

\subsubsection{The AdS/CFT correspondence and composite Higgs bosons in RS Models}

An interesting feature of Randall-Sundrum Models is that they are dual to $4 \mathrm{D}$ conformal field theories by the AdS/CFT correspondence [2] Two theories are considered to be dual if their physical spectra and interactions are the same, even if their underlying dynamics are different According to this correspondence, weakly coupled quantum field theories in an $\mathrm{AdS}_{n}$ spacetıme can be identıfied with a strongly coupled conformal field theory on the $n-1$ dimensional boundary For example, certam Randall-Sundrum models can be shown to be dual to Technicolor [45], a class of models inspired by QCD which establısh EWSB through strong gauge interactions, without the use of a Higgs boson [31]

The AdS/CFT duality is useful in many fields of physics - string theory, QCD, condensed matter - as many CFTs are extremely difficult to calculate because they are strongly coupled and perturbation theory cannot be used The weakly-coupled 5D 
perspective provides a perturbative way of approaching such theories In the context of Randall-Sundrum models, only one side of the duality is known the 5D side As such, the detalls of the strong interactions and their elementary degrees of freedom are unknown, the 5D formalism provides only an effective Lagrangian in which the degrees of freedom are composite particles of the CFT This is similar to the Chiral Effectıve Lagrangian desciption of QCD, in which the degrees of freedom are hadrons rather than quarks

Due to this correspondence, Randall-Sundrum models have become a vehicle for studying the older idea that EWSB could arise from a strongly coupled gauge theory Such theorıes generally divide into two classes Technicolor theories [27, 28, 29, 30, 31] and composite Higgs theories [23, 24, 25, 26] As mentioned above, RS models with bulk fermion field but a TeV brane constrained Higgs are dual to the former [45] Similarly, an RS variation in which the 5D Higgs arises from the fifth component of one of the 5D gauge boson fields - formally known as Gauge-Higgs Unification (GHU) - is dual to a 4D CFT with a composite Higgs boson $[46,3,47]$

From the dual picture, a RS model with GHU is composed of two sectors, an elementary sector and a strongly coupled conformal sector [3] However, the two sectors are not fully independent, but couple to each other As a result, the elementary particle states mix with the composite states, yielding mass eigenstates that are superpositions of both elementary and composite states The degree of compositeness in the $4 \mathrm{D}$ CFT is determined by the location of the particle in the $5 \mathrm{D}$ theory, light partıcles located near the Planck brane - such as SM partıcles - will be mostly elementary, whlle heavier particles near the TeV brane such as KK modes - will be predominantly composite The Higgs particle, however, is a fully composite bound state of the strong sector 
RS models with GHU provide a promising picture of EWSB, as RS models they have all the advantages discussed in the previous section, and they can be formulated so as to obey current electroweak precision tests and experimental constraints [48, $4,49,50]$, the violation of which is a common issue in Technicolor models [27] A common version of GHU is that of the Minimal Composite Higgs Model (MCHM), which was first proposed by $\mathrm{K}$ Agashe, $\mathrm{R}$ Contıno and A Pomarol [3] We will review the details of this model in the following section

\subsection{The Minimal Composite Higgs Model}

For the purposes of this model, we will rewrite the metric, Eq (3 1), in an equivalent form $[3,51]$,

$$
d s^{2}=\frac{1}{(k z)^{2}}\left[\eta_{\mu \nu} d x^{\mu} d x^{\nu}-d z^{2}\right] \equiv g_{M N} d x^{M} d x^{N}
$$

where $M, N=0 \quad 3,5$ are Lorentz indices and $x_{5}=z=\frac{e^{k y}}{k}$ is the coordinate of the fifth dimension The fifth dimension has range $L_{0} \leq z \leq L_{1}$, where $L_{0}=\frac{1}{k} \approx \frac{1}{M_{p}}$ and $L_{1}=\frac{1}{M_{k k}} \approx \frac{1}{T e V} \quad M_{k k}$ denotes the Kaluza-Kleın mass scale

The particles of the model are located in the bulk of the fifth dimension The Higgs is located extremely close to the TeV brane, fermions are near the UV brane, and the gauge bosons are somewhere in between

As discussed in Sec 31 , the primary strength of this model is that it provides a natural solution to the Hierarchy problem through the warped space-time of the fifth dimension [3] Furthermore, placing the fermions in the bulk provides an explanation for the hierarchy of fermion masses, the mass of the fermion will be determined by its overlap with the Higgs in the fifth dimension By placing light fermions very close to the UV brane, but keeping heavier ones such as the top quark nearer to the 
Higgs, the proper spectrum of fermion masses - including those of neutrinos - can be produced [52]. There is also a possibility that the model could give rise to a dark matter candidate [52]. As such, this model has the potential to provide answers to many questions left unanswered by the Standard Model.

\subsubsection{Symmetries of the model}

In the bulk of the five dimensional space, the model has an $S U(3)_{c} \times S O(5) \times U(1)_{B-L}$ gauge symmetry. This symmetry is broken to $S U(3)_{C} \times S U(2)_{L} \times U(1)_{Y}$ on the Planck brane, $z=L_{0}$, and to $S U(3)_{c} \times S O(4) \times U(1)_{B-L}$ on the TeV brane, $z=L_{1}[3,46]$; here $S O(4) \approx S U(2)_{L} \times S U(2)_{R}, Y=Q_{B-L}+T^{3_{R}}$ is the SM hypercharge and $Q_{B-L}$ is the charge corresponding to $U(1)_{B-L} \cdot{ }^{1}$ A schematic representation of the model geometry is given in Fig. 3.1.

We will denote the bosons corresponding to the $U(1)_{B-L}, S O(5)$ symmetries by $U_{M}, A_{M}^{a}$ respectively. The generator index a may take on the values $a=a_{L, R}, \hat{a}$ where $a_{L, R}=1,2,3$ and $\hat{a}=1 . .4$; there are ten in total. The $a_{L, R}$ generators correspond to the $S O(4) \approx S U(2)_{L} \times S U(2)_{R}$ symmetry that remains unbroken on the $\mathrm{TeV}$ brane, while the $\hat{a}$ are generators corresponding to the remaining broken $S O(5) / S O(4)$ symmetry.

The symmetry breaking is achieved by applying appropriate boundary conditions (BCs) to the 4-component gauge fields $A_{\mu}^{a}$; a Dirichlet $\mathrm{BC}(f(z)=0)$ results in a broken gauge symmetry, while a Neumann $\mathrm{BC}\left(\partial_{z} f(z)=0\right)$ preserves the gauge symmetry $[3,51]$. Since we want an unbroken $S U(2)_{L} \times S U(2)_{R} \times U(1)_{B-L}$ symmetry

\footnotetext{
${ }^{1} B-L$ refers to the baryon number $\left(B=\frac{1}{3}\right.$ for a quark) minus the lepton number ( $L=1$ for a lepton).
} 


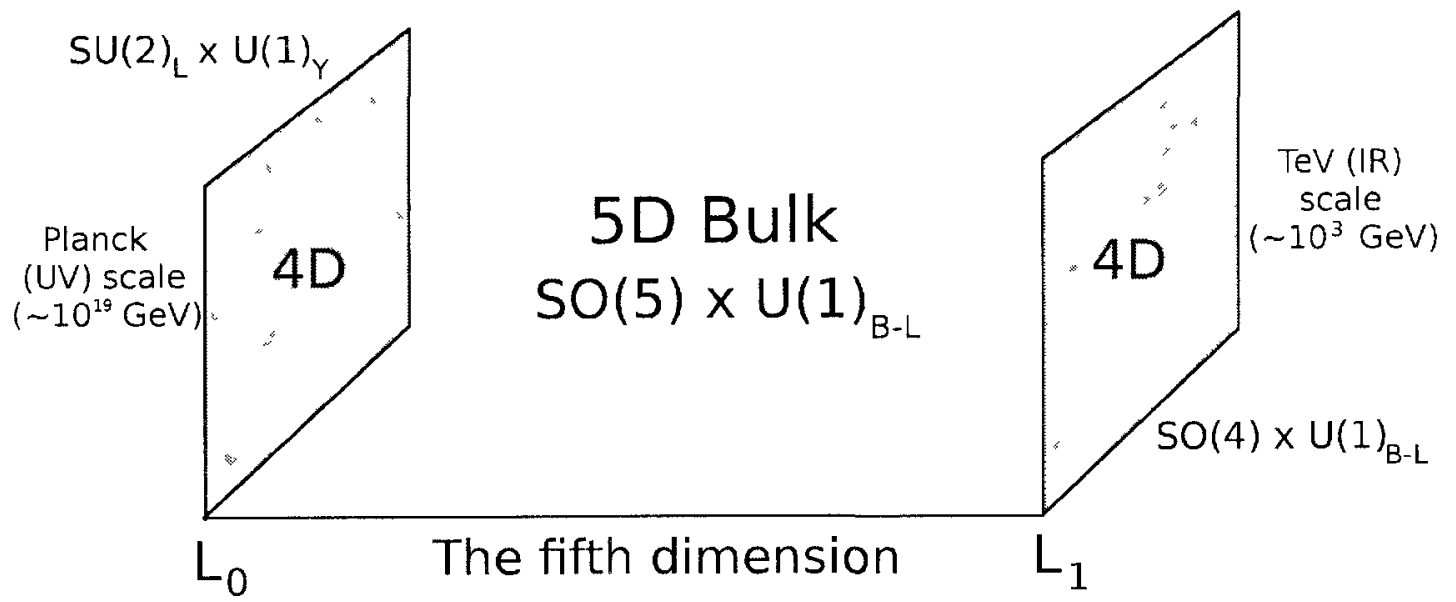

Figure 3.1: A schematic representation of the MCHM spacetime. Note that the symmetry group $S U(3)_{c}$ is unbroken everywhere

on the TeV boundary, both the $A_{\mu}^{a_{L R}}$ and $U_{\mu}$ bosons must have Neumann BCs at $z=L_{1}$, while the $A_{\mu}^{\hat{a}}$ bosons must have Dirichlet BCs. Similarly, at the Planck boundary the $A_{\mu}^{a_{L}}$ must have Neumann BCs to preserve the $S U(2)_{L}$ symmetry. However, on this boundary we only want to preserve $U(1)_{Y}$, not $U(1)_{B-L}$. As such, there will be a linear combination of $U_{\mu}$ and $A_{\mu}^{3_{R}}$ which will correspond to the SM hypercharge boson $B_{\mu}$ and will have Neumann BCs at both boundaries. The opposite linear combination, which we will call $X_{\mu}$, will have a Dirichlet BC at the Planck boundary, but a Neumann BC at the TeV boundary. Finally, $A_{\mu}^{1_{R}}, A_{\mu}^{2_{R}}$ and $A_{\mu}^{\hat{a}}$ bosons will all have Dirichlet BCs at the Planck boundary.

The scalar fifth components automatically must have opposite BCs to the corresponding gauge field $[46,3]$. Therefore $A_{5}^{a_{L}}$ and $B_{5}$ have Dirichlet-Dirıchlet BCs, $A_{5}^{1_{R}}, A_{5}^{2 R}$ and $X_{5}$ have Neumann-Dirichlet BCs, and the $A_{5}^{\hat{a}}$ have Neumann-Neumann BCs. As Neumann-Neumann BCs are required for a zero-mode to arise, only the $A_{\mu}^{a_{L}}$, 
$B_{\mu}$ and $A_{5}^{a}$ bosons will have zero-modes, these will ultimately become our SM gauge bosons and Higgs doublet

\subsubsection{Model Lagrangians}

The electroweak gauge sector of the model is descrıbed by the following Lagrangian [3, $38,51]$

$$
L_{\text {Gauge }}=-\frac{\sqrt{\bar{g}}}{4} g^{M N} g^{R S}\left(F_{M R}^{a} F_{N S}^{a}+U_{M R} U_{N S}\right)+\sqrt{\bar{g}} L_{G F}
$$

where $L_{G F}$ is the gauge fixing term $[46,38]$,

$$
\sqrt{\bar{g}} L_{G F}=-\frac{1}{2 \epsilon k z}\left[\partial^{\mu} A_{\mu}^{a}-\epsilon z \partial_{z}\left(\frac{1}{z} A_{5}^{a}\right)\right]^{2},
$$

and $\epsilon$ is a gauge-fixing pararncter ${ }^{2}$ The stress tensors arc defined as

$$
\begin{aligned}
& F_{M N}^{a}=\partial_{M} A_{N}^{a}-\partial_{N} A_{M}^{a}+g_{5} A_{M N}^{a}, \\
& U_{M N}=\partial_{M} U_{N}-\partial_{N} U_{M}
\end{aligned}
$$

where $A_{M N}^{a} T^{a}=-\imath\left[T^{c}, T^{b}\right]^{a} A_{M}^{c} A_{N}^{b} \quad$ The generators of $S O(5), T^{a}$, are given explicitely in Appendix B

The Lagrangian for a fermion is given by $[3,51]$

$$
L_{f}=\sqrt{\bar{g}}\left[\frac{\imath}{2} \bar{\Psi} e_{A}^{M} \Gamma^{A} D_{M} \Psi-\frac{\imath}{2}\left(D_{M} \Psi\right)^{\dagger} \Gamma^{0} e_{A}^{M} \Gamma^{A} \Psi-M_{\Psi} \bar{\Psi} \Psi\right]
$$

where $e_{A}^{M}=k z \delta_{A}^{M}$ is the vielbein, ${ }^{3}$ the 5 -dimensional Dirac matrices arc $\Gamma^{M}=$

\footnotetext{
${ }^{2}$ Recall that we defincd $\bar{g}$ to be the determinant of the metric $g_{M N}$, it should not be confused with the weak coupling constant $g$ The trace of the metric is $(k z)^{-10}$, therefore $\sqrt{\bar{g}}=(k z)^{-5}$ Note also that we have omitted bulk mass terms in $\mathrm{Eq} 38$, we set them to zero

${ }^{3}$ Note that the vielbein plays the role of the metric here, there is no extra metric factor implied in the contraction of the gamma matrix and the derivative
} 
$\left\{\gamma^{\mu},-r \gamma^{5}\right\}$, and the full covariant derivative is

$$
D_{M}=\partial_{M}+\frac{1}{8} \omega_{M A B}\left[\Gamma^{A}, \Gamma^{B}\right]-\imath g_{5} A_{M}-\imath g_{5}^{\prime \prime} Q_{B-L} U_{M}
$$

where $A_{M}=T^{a} A_{M}^{a}, U_{M}$ is the gauge boson corresoponding to $\mathrm{U}(1)_{B-L}$, and $\omega_{M A B}$ is the spin connection However, it should be noted that in a diagonal metric such as Eq (3 7) the spin-connection terms cancel out in Eq (3 12)

\subsubsection{The gauge boson sector}

As previously discussed, from a four-dımensional perspective our 5D gauge bosons $A_{\mu}^{a}$ correspond to a tower of Kaluza-Klem (KK) modes Standard Model particles correspond to the zero modes of these KK towers, which will only exist if the boson is subject to Neumann-Neumann boundary conditions Before EWSB, all of the zero modes are massless, whle the higher order gauge KK masses are located above the KK mass scale, $M_{k k}=\frac{1}{L_{1}}$, which is defined by the length of the fifth dimension The first gauge KK modes (those of $A_{\mu}^{a_{L R}}$ ) appear at approximately $25 M_{k k}$, and subsequent $K K$ modes of the same boson are separated by roughly $31 M_{k k}$ For a schematic example of the KK spectrum, see Fig (32) near the end of the section

In the case of the MCHM, the zero mode of the $A_{\mu}^{a_{L}} \mathrm{KK}$ tower corresponds to our usual SM $S U(2)_{L}$ bosons, we will therefore rename $A_{\mu}^{a_{L}}$ as the more famllar $W_{\mu}^{a_{L}}$ The $A_{\mu}^{a_{R}}$ bosons arise similarly from the extra $S U(2)_{R}$ symmetry of the MCHM, but have no SM analogue, let us call them $W_{\mu}^{a_{R}}$

As in the Standard Model, the physical states may be obtained by performing rotations on our basis of states The first rotation transforms $\left(W_{\mu}^{3_{R}}, U_{\mu}\right) \rightarrow\left(B_{\mu}, X_{\mu}\right)$ 
through an angle $\theta_{H}$,

$$
\begin{gathered}
W_{\mu}^{3_{R}}=\cos \theta_{H} B_{\mu}-\sin \theta_{H} X_{\mu}, \\
U_{\mu}=\sin \theta_{H} B_{\mu}+\cos \theta_{H} X_{\mu},
\end{gathered}
$$

where $B_{\mu}$ is the SM hypercharge gauge boson, and $X_{\mu}$ is a new gauge boson, their respective BCs have been previously discussed in Sec 321 The mixing angle $\theta_{H}$ is related to the Weinberg angle through the relation $\cos \theta_{H}=\tan \theta_{w}$ This relation is determined by requirıng that the coupling between the physical Higgs - which we will choose to be $A_{5}^{4}$ - and the photon $V_{\mu}$ be zero, as the photon is massless and the Higgs is neutral As a result, both the $Z$ and $X$ couplings to the photon also disappear, indicating that both are neutral bosons The zero mode of $B_{\mu}$ is the analogous particle of the SM, and so our famllar SM states can be found by performing the Standard Model rotation through the Wemberg angle - as described previously by Eqs (2 14) and $(215)$ - to transform $\left(W_{\mu}^{3_{L}}, B_{\mu}\right) \rightarrow\left(Z_{\mu}, V_{\mu}\right)$ Similarly, the tiansformation for $W_{\mu}^{12} \rightarrow W_{\mu}^{ \pm}, \mathrm{Eq}$ (2 13), can be performed on both $W_{\mu}^{a_{L}}$ and $W_{\mu}^{a_{R}}$ To obtain real coupling values it is also convenient to apply an analogous transformation to the $A_{\mu}^{1}$ and $A_{\mu}^{2}$

$$
A_{\mu}^{ \pm}=\frac{1}{\sqrt{2}}\left(A_{\mu}^{1} \mp \imath A_{\mu}^{2}\right)
$$

As a result, from the 4D perspective in the MCHM we have a tower of KK modes with a zero mode for each of the electroweak Standard Model bosons $W_{L \mu}^{ \pm}$, $Z_{\mu}$, and the photon $V_{\mu}$ We also have seven extra gauge KK towers without zero modes due to the extended gauge symmetry $W_{R \mu}^{ \pm}, X_{\mu}, A_{\mu}^{ \pm}, A_{\mu}^{3}$ and $A_{\mu}^{4}$ In addition, each of these towers of vector bosons has an accompanying tower of scalar bosons from the corresponding $A_{5}^{a}$ These are Goldstone bosons, in the unitary gauge each $A_{5}^{a(n)} \mathrm{KK}$ boson becomes the third polarization of its corresponding $A_{\mu}^{a(n)}$, where $n \neq 0$ denotes the $\mathrm{n}^{\text {th }} \mathrm{KK}$ mode 


\begin{tabular}{|c|c|c|}
\hline UV Boundary & TeV Boundary & Particles \\
Condition & Condition & \\
\hline Neumann & Neumann & $W_{L \mu}^{ \pm(n)}, Z_{\mu}^{(n)}, V_{\mu}^{(n)}, H_{5}$ \\
\hline Dirichlet & Neumann & $W_{R \mu}^{ \pm(n)}, X_{\mu}^{(n)}$ \\
\hline Dirichlet & Dirichlet & $A_{\mu}^{ \pm(n)}, A_{\mu}^{\hat{3}(n)}$ and $A_{\mu}^{\hat{4}(n)}$ \\
\hline
\end{tabular}

Table 3.1: The gauge boson spectrum of the MCHM.

However, the $Z_{5}, V_{5}, W_{L, R 5}^{ \pm}$and $X_{5}$ towers have no zero-modes, for they have either Dirichlet-Dirichlet or Neumann-Dirichlet BCs as previously discussed. As only the $A_{5}^{ \pm}, A_{5}^{\hat{3}}$ and $A_{5}^{\hat{4}}$ scalar towers obey Neumann-Neumann BCs, they are the only fifth component towers with zero modes. As such, these four zero-mode scalars are the Goldstone bosons that correspond to our SM zero modes; in other words, they are our Higgs doublet. As in the SM, three of them are eaten by the zero modes of the $W_{L \mu}^{ \pm}$and $Z_{\mu}$ towers - our SM $W_{\mu}^{ \pm}$and $Z_{\mu}$ - when the latter become massive due to EWSB, exactly as in the Standard Model.

In the end, only one scalar boson is left over; it becomes our Higgs boson. It does not matter which three $A_{5}^{\hat{a}(0)}$ bosons become polarizations and which becomes the Higgs; we will choose our Higgs to be the fourth boson, $H_{5}(x, z)=A_{5}^{\hat{4}}$. ${ }^{4}$ This mechanism where the Higgs arises naturally out of the gauge structure of the model is known as Gauge-Higgs Unification. Therefore, in the unitary gauge we are left with the spectrum of bosons outlined in Table 3.1.

\footnotetext{
${ }^{4}$ Here the 5 subscript on the $H$ denotes that this is the 5D Higgs.
} 
As discussed in the previous section, the $5 \mathrm{D}$ bosons are superpositions of these 4D KK modes along with their profile which describes their location in the fifth dimension In the absence of EWSB, this profile $f_{G}(m, z)$ is obtaned from the boson equation of motion which can be derived from the Lagrangian Eq (3 9),

$$
\left[p^{2}-\frac{1}{z} \partial_{z}+\partial_{z}^{2}\right] f_{G}(p, z)=0
$$

where $p=\sqrt{p^{2}}$ is equivalent to the KK mass $[38,51,39]$ It is convenient for future purposes to define even and odd solutions $C_{A}(m, z)$ and $S_{A}(m, z)$ with the boundary conditions $C_{A}\left(m_{n}, L_{0}\right)=1, \partial_{z} C_{A}\left(m_{n}, L_{0}\right)=0, S_{A}\left(m_{n}, L_{0}\right)=0$ and $\partial_{z} S_{A}\left(m_{n}, L_{0}\right)=$ $m_{n},[4,53]$ such that

$$
\begin{aligned}
C_{A}\left(m_{n}, z\right) & =\frac{\pi m_{n}}{2} z\left[J_{1}\left(m_{n} z\right) Y_{0}\left(m_{n} L_{0}\right)-J_{0}\left(m_{n} L_{0}\right) Y_{1}\left(m_{n} z\right)\right] \\
S_{A}\left(m_{n}, z\right) & =\frac{\pi m_{n}}{2} z\left[J_{1}\left(m_{n} L_{0}\right) Y_{1}\left(m_{n} z\right)-J_{1}\left(m_{n} z\right) Y_{1}\left(m_{n} L_{0}\right)\right]
\end{aligned}
$$

where $J_{n}(x)$ and $Y_{n}(x)$ are the $n^{\text {th }}$ order Bessel functions of the first and second kınd, detalls on these functions can be found in Appendix A The profile of a boson with a Neumann (Dirichlet) boundary condition on the Planck brane is given by $C_{A}(m, z)$ $\left(S_{A}(m, z)\right)$ normalızed according to $[51]$

$$
\int_{L_{0}}^{L_{1}} d z \frac{1}{k z} f_{G}^{(n)}(z) f_{G}^{(m)}(z)=\delta_{m n}
$$

The zero mode profile can be determined by solving Eq (3 17) with $p=m_{n}=0$, and imposing the normalization condition, yielding a profile independent of $z$

$$
f_{G}^{(0)}(z)=\sqrt{\frac{k}{\ln \left(k L_{1}\right)}}
$$

The mass spectrum $m_{n}$ of the gauge bosons is determined by imposing the $\mathrm{TeV}$ boundary condition upon the approprıate profile For example, the mass spectrum of a partıcle with a Neumann-Neumann boundary condıtıon will be defined by the 
mass conditıon $C_{A}^{\prime}\left(m, L_{1}\right)=0$ (where the prime indicates the derıvative with respect to $z$ )

Because the Higgs arises as the zero mode of the $A_{5}^{4}$ scalar, its profile does not have the same form as those of the gauge bosons The requirement that zero-mode particles be massless before EWSB leads to the following Higgs profile [3],

$$
f_{H}(z)=z \sqrt{\frac{2 k}{L_{1}^{2}-L_{0}^{2}}}
$$

Note that although this profile is linear in the warped coordinate $z$, in the flat-space coordinate $y$ the profile is extremely peaked toward the TeV brane

\subsubsection{The fermion sector}

To properly incorporate fermions into a 5D model, they must be embedded in an appropriate representation of $S O(5) \times U(1)_{B} L_{L}$ The choice of embedding is important, as it strongly affects the physics of the model $[48,54,49]$ We will make use of the Medina-Shah-Wagner (MSW) embedding [4], as it has been shown to satisfy electroweak precision constraints (EWPCs) $[49,4]^{5}$ In the MSW model, each generation of fermions is embedded in three multiplets, two 5's and one 10 of $S O(5) \times U(1)_{B} L$ The left-handed quark doublet and the right-handed up-type quark singlet are each embedded in a different $\mathbf{5}_{2 / 3}$ representation, while the down-type quark singlet is embedded in a $10_{2 / 3}$ representation (here $2 / 3$ is the $U(1)_{B-L}$ charge) The quark

\footnotetext{
${ }^{5}$ Other common fermion embeddings are the Hosotan Oda-Ohnuma-Sakamura (HOOS) embedding $[55,56]$, and the orıginal MCHM4 spinorial embedding [3], however, in the former the ZZH coupling - which is key to our project - does not exist, and the latter is difficult to reconcle with EWPCs $[48,49]$
} 
embeddings have the form

$$
\begin{aligned}
\xi_{1_{L}}^{q_{2}}= & Q_{1 L}^{2} \oplus \hat{t}_{1_{L}}^{2}=\left(\begin{array}{cc}
\chi_{1_{L}}^{2}(-,+)_{\frac{5}{3}} & t_{1_{L}}^{2}(+,+)_{\frac{2}{3}} \\
\tilde{t}_{1_{L}}^{2}(-,+)_{\frac{2}{3}} & b_{1_{L}}^{2}(+,+)_{-\frac{1}{3}}
\end{array}\right) \oplus \hat{t}_{1_{L}}^{2}(-,+)_{\frac{2}{3}} \\
\xi_{2_{R}}^{q_{2}}= & Q_{2 R}^{2} \oplus \hat{t}_{2_{R}}^{\imath}=\left(\begin{array}{cc}
\chi_{2_{R}}^{2}(-,+)_{\frac{5}{3}} & t_{2_{R}}^{2}(-,+)_{\frac{2}{3}} \\
\tilde{t}_{2_{R}}^{2}(-,+)_{\frac{2}{3}} & b_{2_{R}}^{2}(-,+)_{-\frac{1}{3}}
\end{array}\right) \oplus \hat{t}_{2_{R}}^{\imath}(+,+)_{\frac{2}{3}} \\
\xi_{3_{R}}^{q_{2}}= & T_{1 R}^{2} \oplus T_{2 R}^{2} \oplus Q_{3 R}^{2}\left(\begin{array}{c}
\Xi_{3_{R}}^{2}(-,+)_{\frac{5}{3}} \\
T_{3_{R}}^{2}(-,+)_{\frac{2}{3}} \\
B_{3_{R}}^{2}(-,+)_{-\frac{1}{3}}
\end{array}\right) \oplus\left(\begin{array}{c}
\Xi_{3_{R}}^{\prime 2}(-,+)_{\frac{5}{3}} \\
T_{3_{R}^{\prime}}^{\prime \imath}(-,+)_{\frac{2}{3}} \\
B_{3_{R}}^{\prime \prime}(+,+)_{-\frac{1}{3}}
\end{array}\right) \oplus\left(\begin{array}{ll}
\chi_{3_{R}}^{2}(-,+)_{\frac{5}{3}} & t_{3_{R}}^{2}(-,+)_{\frac{2}{3}} \\
\tilde{t}_{3_{R}}^{2}(-,+)_{\frac{2}{3}} & b_{3_{R}}^{2}(-,+)_{-\frac{1}{3}}
\end{array}\right)
\end{aligned}
$$

where $\hat{t}$ denotes an $S U(2)_{L} \times S U(2)_{R}$ singlet, $T_{1}$ and $T_{2}$ transform under $(3,1)$ and $(1,3)$ of $S U(2)_{L} \times S U(2)_{R}$, and $Q$ 's denote bidoublets of $S U(2)_{L} \times S U(2)_{R}$ (where $S U(2)_{L}$ acts vertically and $S U(2)_{R}$ acts horizontally) $[4,50]$. The final subscripts denote the electromagnetic charge, while the superscripts $i=1,2,3$ denote the generation. The plus and minus signs represent even and odd boundary conditions respectively; the first entry corresponds to the Planck brane boundary condition, while the second corresponds to the $\mathrm{TeV}$ brane boundary condition. For fermions an odd boundary condition is the usual Dirichlet condition, but an even BC is a superposition of Dirichlet and Neumann BCs. However, fermions with even BCs $(++)$ on both boundaries still correspond to SM particles. In the case of a quark multiplet, $t_{1_{L}}$ and $b_{1_{L}}$ together correspond to the left-handed SM doublet of that generation, $t_{1_{L}}$ being the up-type quark and $b_{1_{L}}$ being the down-type quark. Similarly, $\hat{t}_{2_{R}}$ and $B_{3_{R}}^{\prime}$ correspond to the right-handed up and down-type quark singlets respectively.

It should be noted that in general 5D fermion fields are inherently non-chiral 
Dirac spinors. Right- and left-handed Weyl spinors may be obtained from these Dirac spinors due to the $Z_{2}$ orbifold symmetry that is imposed on the fifth dimension, as discussed in Sec. 3.1.1 [40]. Fermions transform as $\Psi(-y)= \pm \gamma_{5} \Psi(y)$ under this symmetry, allowing the identification of right- and left-handed chiral states, $\Psi_{L, R}=$ $\pm \gamma_{5} \Psi_{L, R}$. One of these chiral states will have an odd boundary condition, while the other will have an even boundary condition; as such, the boundary conditions of the multiplet of opposite chirality can be obtained simply by reversing the chiralities and boundary conditions in Eqs. (3.23-3.25).

The leptons are embedded in similar representations; the doublets and righthanded neutrinos are embedded in $\mathbf{5}_{\mathbf{0}}$ representations, while the right-handed lepton singlets are embedded in $10_{0}$ representations [52],

$$
\begin{aligned}
& \xi_{1_{L}}^{\ell_{2}}=Q_{1 L}^{\ell_{2}} \oplus \hat{n}_{1_{L}}^{2}=\left(\begin{array}{cc}
\kappa_{1_{L}}^{\imath}(-,+)_{1} & n_{1_{L}}^{\imath}(+,+)_{0} \\
\tilde{n}_{1_{L}}^{2}(-,+)_{0} & \ell_{1_{L}}^{\imath_{L}}(+,+)_{-1}
\end{array}\right) \oplus \hat{n}_{1_{L}}^{2}(-,+)_{0} \\
& \xi_{2_{R}}^{\ell_{2}}=Q_{2 R}^{\ell_{2}} \oplus \hat{n}_{2_{R}}^{2}=\left(\begin{array}{cc}
\kappa_{2_{R}}^{2}(-,+)_{1} & n_{2_{R}}^{2}(-,+)_{0} \\
\tilde{n}_{2_{R}}^{2}(-,+)_{0} & \ell_{2_{R}}^{2}(-,+)_{-1}
\end{array}\right) \oplus \hat{n}_{2_{R}}^{2}(+,+)_{0} \\
& \xi_{3_{R}}^{\ell_{2}}=T_{1 R}^{\ell_{2}} \oplus T_{2 R}^{\ell_{2}} \oplus Q_{3 R}^{\ell_{2}} \\
& =\left(\begin{array}{c}
K_{3_{R}}^{\imath}(-,+)_{1} \\
N_{3_{R}}^{\imath}(-,+)_{0} \\
L_{3_{R}}^{2}(-,+)_{-1}
\end{array}\right) \oplus\left(\begin{array}{c}
K_{3_{R}}^{\prime \imath}(-,+)_{1} \\
N_{3_{R}}^{\prime}(-,+)_{0} \\
L_{3_{R}{ }^{\imath}}(+,+)_{-1}
\end{array}\right) \oplus\left(\begin{array}{ll}
\kappa_{3_{R}}^{2}(-,+)_{1} & n_{3_{R}}^{\imath}(-,+)_{0} \\
\tilde{n}_{3_{R}}^{2}(-,+)_{0} & \ell_{3_{R}}^{2}(-,+)_{-1}
\end{array}\right)
\end{aligned}
$$

where $\hat{n}$ denotes an $S U(2)_{L} \times S U(2)_{R}$ singlet, and $T_{l}, Q_{\imath}$ transform as in the quark multiplets. Similarly to the quark case, $\ell_{1_{L}}, n_{1_{L}}\left(L_{3_{R}}^{\prime}, \hat{n}_{2_{R}}\right)$ is the left-handed (righthanded) SM lepton and its associated neutrino. 
Similarly to the boson case, in the absence of EWSB, the fermion profiles of left and right chirality, $f_{L, R}(m, z)$ are solutions to

$$
\left[\partial_{z}-z^{-1}(2 \pm c)\right]\left[\partial_{z}-z^{-1}(2 \mp c)\right] f_{L, R}^{(n)}(z)=-m_{n}^{2} f_{L, R}^{(n)} .
$$

which can be derived from the Lagrangian Eq. (3.12) [51]. The parameter $c$ determines the location of the fermion along the fifth dimension, and is defined in terms of the fermion bulk mass $M_{\Psi}$ by $M_{\Psi}=c k$. Note that there are three $c$ values $\left(c_{1}^{2}, c_{2}^{2}, c_{3}^{2}\right)$ for each generation of quarks and each generation of leptons, one for each multiplet $\xi_{1}^{2}, \xi_{2}^{2}, \xi_{3}^{2}$. We define the following solution to Eq. $3.29[4,50]$ :

$S_{c}^{ \pm}\left(m_{n}, z\right)=\frac{\pi m_{n}}{2 k}(k z)^{\frac{5}{2}}\left[J_{ \pm c+\frac{1}{2}}\left(m_{n} L_{0}\right) Y_{ \pm c+\frac{1}{2}}\left(m_{n} z\right)-J_{ \pm c+\frac{1}{2}}\left(m_{n} z\right) Y_{ \pm c+\frac{1}{2}}\left(m_{n} L_{0}\right)\right]$.

Up to a normalization constant, $S_{c}^{+}\left(m_{n}, z\right)\left(S_{c}^{-}\left(m_{n}, z\right)\right)$ is the profile of a left-handed (right-handed) fermion with a Dirichlet boundary condition on the Planck brane. The corresponding chiral partners have a Neumann BC - a mixed BC for fermions - on the Planck brane, and have profiles defined by $[51,4,50]$

$$
\dot{S}_{c}^{ \pm}\left(m_{n}, z\right)=\mp \frac{1}{m_{n}}\left[\partial_{z}-\frac{1}{z}(2 \mp c)\right] S_{c}^{ \pm}\left(m_{n}, z\right) .
$$

Please see Table 3.2 for a summary of the functions associated with the profiles of different chirality. Again, the fermion mass spectrum $m_{n}$ is defined by imposing the $\mathrm{TeV}$ boundary condition upon the profiles above.

The fermion profiles must satisfy the following normalization condition [51]

$$
\int_{L_{0}}^{L_{1}} d z \frac{1}{(k z)^{4}} f_{f}^{(n)}(z) f_{f}^{(m)}(z)=\delta_{m n}
$$

By solving Eq. (3.29) with $m_{n}=0$ and imposing the normalization condition, one obtains the zero-mode profile

$$
f_{L}^{(0)}(z)=\sqrt{\frac{k(1-2 c)}{\left(k L_{1}\right)^{1-2 c}-1}}(k z)^{2-c} .
$$




\begin{tabular}{|c|c|c|}
\hline $\begin{array}{c}\text { Boundary Condition } \\
\text { at the Planck Brane }\end{array}$ & Left-handed & Right-handed \\
fermion & fermion \\
\hline Dirıchlet & $S_{c}^{+}\left(m_{n}, z\right)$ & $S_{c}^{-}\left(m_{n}, z\right)$ \\
\hline Neumann & $S_{c}^{-}\left(m_{n}, z\right)$ & $S_{c}^{+}\left(m_{n}, z\right)$ \\
\hline
\end{tabular}

Table 32 Profiles associated to fermion chiralitıes with different BCs

It is easier to see from $\mathrm{Eq}$ (3 33) how the parameter $c$ determines the location of the zero-mode fermion profile, as the power of the warped coordinate $z$ is determmed by $c$ If $c>05(c<05)$ the fermon's profile is angled toward the Planck (TeV) branc, while if $c=05$ the profile is flat As such, light fermons require $c>05$, while the heavier top and bottom quarks are assigned $c<05$ It should also be noted that when we speak of the profile being flat, it is with respect to the flat-space coor dinate $y$, not the warped-space coordinate $z$

Fermions are also subject to an $S U(2)_{L} \times S U(2)_{R} \times U(1)_{B-L}$ invariant boundary mass Lagrangian on the $\mathrm{TeV}$ brane, which marries the $S U(2)_{L} \times S U(2)_{R}$ bidoublets and singlets via Dirac mass terms on the TeV brane and introduces mixing between fermion states of the same electromagnetic charge [3] In the case of leptons, Majorana mass terms for the right-handed neutrino $N_{R}$ may be added to either boundary The lepton boundary mass Lagrangian takes the general form

$$
\begin{aligned}
L_{\mathrm{bound}}= & -2 \delta\left(z-L_{1}\right)\left[\bar{N}_{L}^{\prime} M_{\ell_{1}} N_{R}+\bar{L}_{1 L} M_{\ell_{2}} L_{3 R}+\mathrm{hc}\right] \\
& -\left[M_{I R} \delta\left(z-L_{1}\right)-M_{U V} \delta(z)\right] N_{R} N_{R}
\end{aligned}
$$

where $M_{\ell_{12}}$ are dimensionless matrices of the Dirac mass terms [52] $M_{I R}\left(M_{U V}\right)$ 
denotes the Majorana mass term on the TeV (Planck) brane, and is also dimensionless

The quark mass Lagrangian and boundary conditions are completely analogous If the Majorana mass terms are set to zero $[4,50]$,

$$
L_{\text {bound }}=-2 \delta\left(z-L_{1}\right)\left[\bar{u}_{L}^{\prime} M_{q_{1}} u_{R}+\bar{Q}_{1 L} M_{q_{2}} Q_{3 R}+\mathrm{h} \mathrm{c}\right]
$$

Similarly to the $c$ parameter, there is a different set of $M_{\ell_{1}}^{\imath}, M_{\ell_{2}}^{\imath}$ (or $M_{q_{1}}^{\imath}, M_{q_{2}}^{\imath}$ ) for each generation of quarks or fermons

These boundary mass terms define the boundary conditions of the fermons Consıder the generıc Lagrangıan terms

$$
L=-2 \delta\left(z-L_{1}\right) \bar{\Psi}_{L}^{1} M \Psi_{R}^{2}-\left[M_{I R} \delta\left(z-L_{1}\right)-M_{U V} \delta\left(z-L_{0}\right)\right] \bar{\Psi}_{R}^{2} \Psi_{R}^{2}
$$

where superscripts 1,2 merely denote two different fermion fields and the different chiralities $\Psi_{L}^{1}, \Psi_{R}^{1}, \Psi_{R}^{2}$ and $\Psi_{L}^{2}$ have profiles $g_{L}(z), g_{R}(z), h_{R}(z)$ and $h_{L}(z)$ respectively Considering Eq (3 36) in the equations of motion leads to the following BCs on the fermion profiles $[50,52]$

$$
\begin{aligned}
& \lim _{\epsilon \rightarrow 0} g_{R}\left(L_{1}-c\right)=-M h_{R}\left(L_{1}\right), \\
& \lim _{\epsilon \rightarrow 0} h_{L}\left(L_{1}-\epsilon\right)=M_{I R} h_{R}\left(L_{1}\right)+M g_{L}\left(L_{1}\right), \\
& \lim _{\epsilon \rightarrow 0} h_{L}\left(L_{0}-\epsilon\right)=M_{U V} h_{R}\left(L_{0}\right)
\end{aligned}
$$

Although the MCHM evidently contains many more fermons than the Standard Model, only those identıfied with the SM - identıfied by $(+,+)$ BCs - will have zero modes The rest exist only as heavy Kaluza-Kleın modes, as in the gauge sector, and so do not significantly affect the physics at the SM particle mass scale However, they do contribute to physics above the KK mass scale, particularly to the KK boson decay widths 


\subsubsection{Electroweak Symmetry Breaking in the MCHM}

In the SM, the Higgs boson only couples $Z$ bosons to $Z$ bosons and $W^{+}$bosons to $W^{-}$bosons. However, in the MCHM the Higgs can couple to different bosons which are not each others' anti-particles. The possible Higgs coupling forms are summarized in Table 3.3.

Table 3.3: Coupling terms that lead to boson mixing after EWSB.

\begin{tabular}{|c|c|c|}
\hline Sector & Bosons & Possible Mixing \\
Index & Involved & Coupling Forms \\
\hline 1 & $W^{1_{L}}, W^{1_{R}}, A^{\hat{1}}$ & $W^{1_{L}} W^{1_{R}} H H, W^{1_{L}} A^{\hat{1}} H, W^{1_{R}} A^{\hat{1}} H$ \\
\hline 2 & $W^{2_{L}}, W^{2_{R}}, A^{\hat{2}}$ & $W^{L_{L}} W^{2_{R}} H H, W^{2_{L}} A^{\hat{2}} H, W^{2_{R}} A^{\hat{2}} H$ \\
\hline 3 & $Z, X, A^{\hat{3}}$ & $Z X H H, Z A^{\hat{3}} H, X A^{\hat{3}} H$ \\
\hline
\end{tabular}

For clarity, the first two groups are left in the original basis; in the physical basis, these two groups would be combined due to rotations of the form $\left(W^{1}, W^{2}\right) \rightarrow\left(W^{+}, W^{-}\right)$. The third group is given in the physical basis, as the corresponding original basis would also include the photon, which does not couple to neutral particles.

Because of these new couplings, the same EWSB mechanism that gives the zero-mode gauge bosons masses also causes certain particles to mix together. For example, consider the coupling between a $\mathrm{Z}$ an $\mathrm{X}$ and two Higgs, $c H H Z_{\mu} X^{\mu}$, where $c$ is the coupling constant. If we let $H \rightarrow v$, we obtain $c v^{2} Z_{\mu} X^{\mu}$. Physically, this term seems to describe the spontaneous transformation of a $Z$ boson into an $X$ boson, 
which does not make sense However, what this term actually indicates is that the $\mathrm{Z}$ boson after EWSB is not the same as the original $Z$, it is a superposition of the original $Z$ and $X$ bosons In fact, due to the couplings in sector three of Table 33 , the $Z$ after EWSB is actually found to be a superposition of the original $Z, X$ and $A^{3}$ bosons, ${ }^{6}$

$$
Z \rightarrow Z_{\mu}(m, z, v)=a Z_{\mu}(m, z, 0)+b X_{\mu}(m, z, 0)+c A_{\mu}^{3}(m, z, 0)
$$

where $a \gg b, c$ It is the coefficients $a, b, c$ that introduce the dependence on the Higgs vev into the profile

Of course, the dominant contribution to $Z^{\prime}$ is the original $Z$, one would expect the contributions of $X$ and $A^{3}$ to be substantially smaller Furthermore, the $X$ and $A^{3}$ bosons after EWSB are also superpositions of the original $Z, X$ and $A^{3}$, with their own original states dommating over the other contributions Similarly, $\left(W^{1_{L}}, W^{1_{R}}\right.$, $\left.A^{1}\right)$ and $\left(W^{2_{L}}, W^{2_{R}}, A^{2}\right)$ also form groups of bosons that becomes superpositions of each other after EWSB, due to the couplings outlined in the first two mixing sectors of Table 33

This mixing of boson states alters the masses of some of the mixed bosons For example, after EWSB the mass conditions of the $Z$ and the $A^{3}$ bosons become related through the Higgs vev, this has the result of shiftıng the $Z \mathrm{KK}$ mode masses down slightly, while the $A^{3}$ KK mode masses are shifted slightly up Furthermore, both bosons are described by the same profile evaluated at different mass values On the other hand, the $X$ mass condition remains unchanged and independent Fig (32) gives a rough picture of the KK mass spectrum of the third mixing sector both before and after EWSB

\footnotetext{
${ }^{6}$ The origin of the $A^{3}$ mixing is somewhat clarified if one recalls that it is the zero mode fifth component $A_{5}^{3(0)}$ that acts as the neutral Goldstone which becomes the $Z$ boson's third polariLation
} 


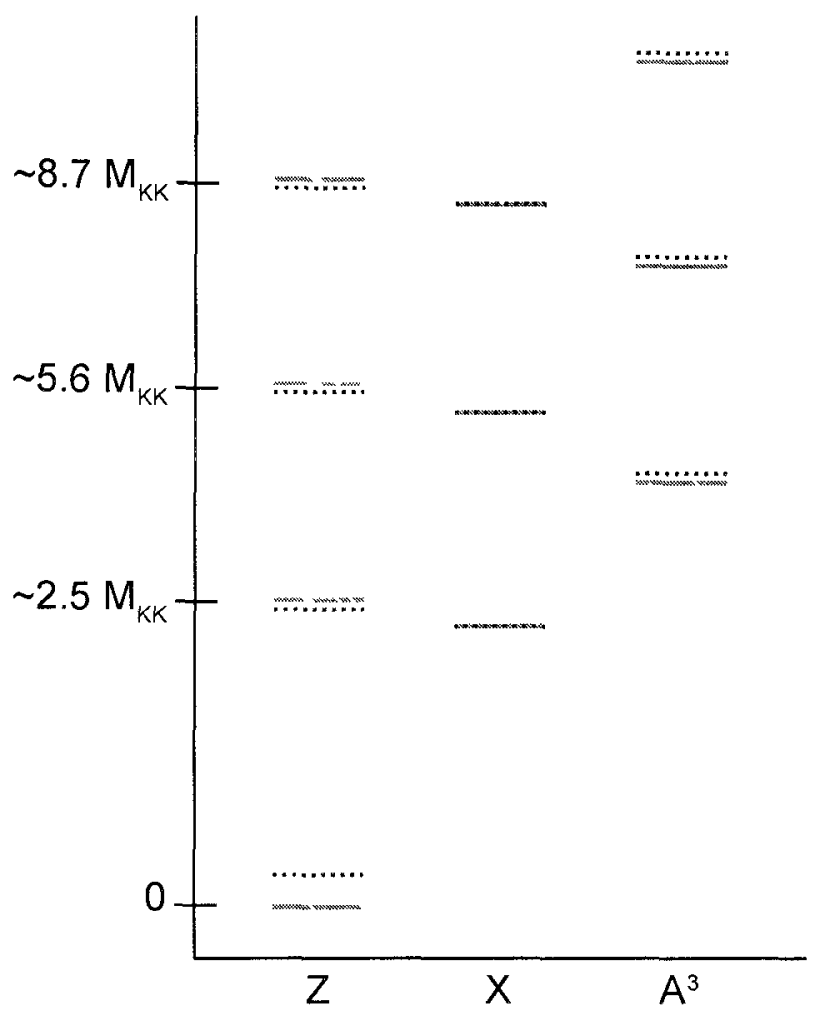

Figure 3.2: A schematic of the KK mode mass distributions for the $Z, X$ and $A^{\hat{3}}$ bosons both before (solid grey lines) and after (black dotted lines) EWSB. Numeric values for a particular parameter set can be found in Table 3.8 in Sec. 3.2.7. Not to scale.

For the purposes of further discussion, we define the following terms

Mixed : Groups of bosons whose profiles after EWSB are linear superpositions of their original profiles.

Unmixed : Bosons whose profiles after EWSB are the same as their original profiles.

Combined : Mixed bosons which are described by the same mass condition and profile after EWSB. 
Independent Mixed bosons with the same mass condition before and after EWSB

In the interests of space and clarity, we will simply summarize the procedure and effects of particle mixing heie A detalled overview of the calculations, transformations, and profiles discussed in this section is provided in Appendix C 3

\section{Gauge Boson Mixing}

The full mixing of gauge bosons through the Higgs vev $v$ after EWSB would be difficult to achieve by considerng KK profiles individually Fortunately, the fully mixed vev-dependent particle profiles $f^{a}(m, z, v)$ can be related to the pre-EWSB profiles $f^{a}(m, z, 0)$ by means of the following gauge transformation $[53,4]$,

$$
f^{a}(m, z, v) T^{a}=\Omega^{-1}(z, v) f^{a}(m, z, 0) T^{a} \Omega(z, v)
$$

where $a=a_{L R}, \hat{a}$, the transformation matrix is

$$
\Omega(z, v)=\exp \left[-\imath g_{5} v \int_{L_{0}}^{z} d z^{\prime} f_{h}\left(z^{\prime}\right)\right]=\exp \left(-\imath \sqrt{2} \theta_{G}(z, v) T^{4}\right)
$$

and

$$
\theta_{G}(z, v)=\frac{v g_{5}\left(z^{2}-L_{0}^{2}\right)}{2} \sqrt{\frac{k}{L_{1}^{2}-L_{0}^{2}}}
$$

The $f_{\alpha}(m, z, 0)$ are respectively defined by Eq (C 21) in terms of the base functions $C_{A}\left(m_{n}, z\right)$ and $S_{A}\left(m_{n}, z\right)$ of Eqs (3 18-3 19) and normalization coefficients $C_{\alpha}$ The resulting EWSB profiles $f_{\alpha}(m, z, v)$ can be found in Eqs (C 47-C 54)

This gauge transformation 'turns on' a non-zero vev $v$ for the Higgs This can be seen by applying the gauge transformation Eq (3 42) to the Higgs $H_{5}=H f_{H}$ in 
analogy with Eq (2 1),

$$
\begin{aligned}
H_{5} T^{4} & \rightarrow \Omega H_{5} T^{4} \Omega^{\dagger}+\frac{\imath}{g_{5}} \Omega \partial_{z} \Omega^{\dagger} \\
& \rightarrow \Omega H f_{H} T^{4} \Omega^{\dagger}+\frac{\imath}{g_{5}} \Omega\left(-\imath g_{5} v f_{H}\right) \Omega^{\dagger}=\Omega(H+v) f_{H} T^{4} \Omega^{\dagger}
\end{aligned}
$$

where for simplicity we have dropped the coordinate dependence in the notation It 1s also interesting to note that $\theta_{G}\left(L_{0}\right)=0$ and therefore $\Omega\left(L_{0}, v\right)=1$, as such, the mixing will not affect particles located near the Planck brane as strongly as those located near the $\mathrm{TeV}$ brane

We may determine both the coefficients $C_{\alpha}$ - up to an overall normalization factor - and the masses $m_{n}$ by applying the boundary conditions at the $\mathrm{TeV}$ brane There are as many $\mathrm{TeV}$ boundary conditions as there are coefficients $C_{\alpha}$, therefore the BCs provide us with a system of equations by which the coefficients may be determined However, this system of equations will only have a solution if its determinant is zero, this extra condition results in the following mass conditions

$$
\begin{gathered}
S_{A}\left(m_{n}, L_{1}\right)=0, \\
C_{A}^{\prime}\left(m_{n}, L_{1}\right)=0, \\
S_{A}^{\prime 2}\left(m_{n} L_{1}\right)=0, \\
2 C_{A}^{\prime}\left(m_{n}, L_{1}\right) S_{A}\left(m_{n} L_{1}\right)+m_{n} k L_{1} \sin \theta_{G}^{2}\left(L_{1}, v\right)=0, \\
2 C_{A}^{\prime}\left(m_{n}, L_{1}\right) S_{A}\left(m_{n}, L_{1}\right)+m_{n} k L_{1} \sec ^{2} \theta_{w} \sin \theta_{G}^{2}\left(L_{1}, v\right)=0,
\end{gathered}
$$

where a prime represents the derivative with respect to the fifth coordinate $z$ Each of these conditions determines the mass parameters $m_{n}$ for a given boson The first two correspond to the $A_{\mu}^{4}$ boson and photon $V_{\mu}$ masses, while the third corresponds to the $W_{R \mu}^{ \pm}$and $X_{\mu}$ masses, all of these particles retain the same mass spectrum as before EWSB However, masses of both the $Z_{\mu}$ and $A_{\mu}^{3}$ boson are described by the fourth 
mass condition, while the fifth describes the $W_{L_{\mu}}^{ \pm}$and $A_{\mu}^{ \pm}$. The vev dependence of these combined mass conditions shifts the associated masses slightly.

Imposing each of these mass conditions on the system of equations yields a separate solution for the coefficients. Although all 10 equations - one for each boson -can be solved together, it is physically illuminating to note that the profile boundary conditions can be broken into four independent systems of equations containing a common set of coefficients $C_{\alpha}$ after EWSB. The particles within each group are listed in the first row of Table 3.4; the second row lists the coefficients to be determined by the corresponding system of equations. As one might expect, they reflect the mixing groups given by Table 3.3 .

Table 3.4: Sectors of gauge bosons mixed by EWSB through the Higgs vev $v$. The third group of particles is given in the physical basis because it is in this basis that the boundary conditions are imposed.

\begin{tabular}{|c||c|c|c|c|}
\hline Group & 1 & 2 & 3 & 4 \\
\hline Particles & $W_{\mu}^{1_{L}}, W_{\mu}^{1_{R}}, A_{\mu}^{\hat{1}}$ & $W_{\mu}^{2_{L}}, W_{\mu}^{2_{R}}, A_{\mu}^{\hat{2}}$ & $Z_{\mu}, X_{\mu}, V_{\mu}, A_{\mu}^{\hat{3}}$ & $A_{\mu}^{\hat{4}}$ \\
\hline Coefficients & $C_{1_{L}}, C_{1_{R}}, C_{\hat{1}}$ & $C_{2_{L}}, C_{2_{R}}, C_{\hat{2}}$ & $C_{3_{L}}, C_{3_{R}}, C_{\hat{3}}, C_{B}$ & $C_{\hat{4}}$ \\
\hline \hline Mixed Bosons & $W_{\mu}^{1_{L}}, W_{\mu}^{1_{R}}, A_{\mu}^{\hat{1}}$ & $W_{\mu}^{2_{L}}, W_{\mu}^{2_{R}}, A_{\mu}^{\hat{2}}$ & $Z_{\mu}, X_{\mu}, A_{\mu}^{\hat{3}}$ & $A_{\mu}^{\hat{4}}$ \\
\hline Combined Bosons & $W_{\mu}^{1_{L}}, A_{\mu}^{\hat{1}}$ & $W_{\mu}^{2_{L}}, A_{\mu}^{\hat{2}}$ & $Z_{\mu}, A_{\mu}^{\hat{3}}$ & - \\
\hline Independent Bosons & $W_{\mu}^{1_{R}}$ & $W_{\mu}^{2_{R}}$ & $X_{\mu}$ & - \\
\hline Unmixed Bosons & - & - & $V_{\mu}$ & $A_{\mu}^{\hat{4}}$ \\
\hline
\end{tabular}


Although the photon $V_{\mu}$ is contained in the third mixing group, it turns out that it is in fact an unmixed particle. When the system of equations corresponding to group 3 is solved under the constraint of the photon mass condition, the resulting coefficients remove the vev dependence from the photon's EWSB profile $f^{V}(m, z ; v)$. Furthermore, the photon coefficients also force $f^{Z}(m, z ; v), f^{X}(m, z ; v)$, and $f^{\hat{3}}(m, z ; v)$ to be zero, while the coefficients associated with the $X, Z$ and $A^{\hat{3}}$ bosons force $f^{V}(m, z ; v)$ to be zero. As such $f^{V}(m, z ; v)=f^{V}(m, z ; 0)$.

However, the masses and coefficients corresponding to the $X$ boson do not force the $Z$ and $A^{\hat{3}}$ profiles to be zero, or vice versa. This indicates that the mass eigenstate is not simply given by the mixed profile $f(z ; v)$ with the appropriate coefficients. The profile of the true mass eigenstate is defined as a superposition of the mixed states weighted by their generators; the generic form is

$$
F_{G}(m, z ; v) T^{F_{G}}=\sum_{\alpha} f_{\alpha}^{G}(m, z ; v) T^{\alpha},
$$

where $\alpha$ runs over the particles contained in the mixing group and $G$ indicates that the mixed profiles within the expression have been evaluated with the coefficients associated with that particle $G$ 's mass condition. The normalization condition for such a profile is given by

$$
\int_{L_{0}}^{L_{1}} \frac{d z}{k z} \sum_{\alpha} f_{\alpha}^{G}\left(m_{n}, z ; v\right) f_{\alpha}^{G}\left(m_{m}, z ; v\right)=\delta_{m n} .
$$

As such, both $Z$ and $A^{\hat{3}}$ are described by the same $F_{G}(m, z ; v)$, as they are associated with the same mass condition and coefficient set. We can therefore see that what we identified as separate $Z$ and $A^{\hat{3}}$ bosons before EWSB have mathematically become combined into a single 5D boson after EWSB (although the resulting KK modes can still be identified with the original $Z$ and $A^{\hat{3}} \mathrm{KK}$ modes based on their masses). The bosons of mixing groups 1 and 2 behave similarly, while $A^{\hat{4}}$ never mixes at all due to 
the fact that its generator commutes with the mixing transformation.

As one might expect, the primary influence of this mixing manifests through the particle couplings. Due to the form of Eq. (3.46), the coupling of a given boson to other particles no longer involves solely its own generator, but also the generators of the bosons with which it is mixed. Consider the coupling of two gauge bosons, $G_{1}$ and $G_{2}$, through a Higgs. Suppose that each is a superposition of two states; $G_{1}$ of states $A$ and $B, G_{2}$ of states $C$ and $D$. Their coupling through the Higgs will be the sum of all possible couplings between their components

$$
\begin{gathered}
G_{1}\left(m_{1}\right) G_{2}\left(m_{2}\right) H \rightarrow C_{A C H} f_{A}^{G_{1}}\left(m_{1}\right) f_{C}^{G_{2}}\left(m_{2}\right) f_{H}+C_{A D H} f_{A}^{G_{1}}\left(m_{1}\right) f_{D}^{G_{2}}\left(m_{2}\right) f_{H} \\
+C_{B C H} f_{B}^{G_{1}}\left(m_{1}\right) f_{C}^{G_{2}}\left(m_{2}\right) f_{H}+C_{B D H} f_{B}^{G_{1}}\left(m_{1}\right) f_{D}^{G_{2}}\left(m_{2}\right) f_{H},
\end{gathered}
$$

where the $C_{A B C}$ 's represent the usual coupling cocfficient between particles $A, B$, $C$ identificd by generators $T^{A}, T^{B}, T^{C}$. The proceedure is the same regardless of the number of mixed bosons involved in the coupling or the number of superimposed states per boson.

This complication of the couplings produces significant differences compared to the pre-EWSB couplings, as well as new couplings which were not previously allowed. For example, before EWSB a $Z$ boson could not couple $W_{L}^{+}$and $W_{R}^{-}$to each other; however, after EWSB $W_{L}^{+}\left(W_{R}^{-}\right)$contains a small component of $W_{R}^{+}\left(W_{L}^{-}\right)$, which couples through the $Z$ to $W_{R}^{-}\left(W_{L}^{+}\right)$, allowing the coupling to be non-zero. Similarly, after EWSB the KK $Z Z H$ couplings contain a contribution from $Z A^{\hat{3}} H$ which significantly alters their value. However, it is worth noting that couplings involving zero modes - SM particles - are only slightly shifted by particle mixing; the dominant effects occur in couplings between KK modes. Furthermore, the profiles themselves are not substantially altered from those before EWSB. Although one cannot plot the 
EWSB profile $F_{G}(m, z ; v)$ due to the generator dependence of its definition, the profile normalization integrands before and after EWSB are indistinguishable when plotted together; in general the relative difference is of a few percent.

\section{Fermion Mixing}

The inter-mixing of fermion states through EWSB can also be accounted for via a similar gauge transformation on each of the fermion multiplets. The multiplets must each be written in vector form, with lepton multiplets $\xi_{\ell_{1 L}}, \xi_{\ell_{2 R}}$ and $\xi_{\ell_{3 R}}$ corresponding to profile vectors $f_{\ell_{1 L}}, f_{\ell_{2}}$ and $f_{\ell_{3}}$, and similarly for quarks The vectors arc defined by Eq. (C.81) in terms of the fermion profilo base functions $S_{c}^{ \pm}$and $\dot{S}_{c}^{ \pm}-$Eqs. (3.303.31)) - and normalization coefficients $C_{f}$. However, these vectors are not defined in the same basis as our generators; to apply the gauge transformation, we must transform the fermion vector to the generator basis, and then transform back to the fermion basis so that boundary conditions can be applied. As such, our lepton profiles after EWSB are related to those before EWSB by

$$
\begin{aligned}
& f_{\ell_{1 L}}\left(m_{n}, z ; v\right)=A \Omega(z, v) A^{-1} f_{\ell_{1 L}}\left(m_{n}, z ; 0\right) \\
& f_{\ell_{2 R}}\left(m_{n}, z ; v\right)=A \Omega(z, v) A^{-1} f_{\ell_{2 R}}\left(m_{n}, z ; 0\right), \\
& f_{\ell_{3 R}}\left(m_{n}, z ; v\right)=B \Omega(z, v) B^{-1} f_{\ell_{3 R}}\left(m_{n}, z ; 0\right)
\end{aligned}
$$

where $\Omega(z, v)$ is defined above in Eq. (3.42) and the change-of-basis matrices $A$ and $B$ are given in Eq. (C.87) and Eq. (C.88) of Appendix C.3.

As in the boson case, we may determine the mass conditions of our mixed fermions by requiring that the determinant of the system of equations given by the fermion boundary conditions, Eqs. (3.37-3.39), be zero, and each mass condition will be associated with a particular solution set of coefficients $C_{f}$. As the boundary 
conditions of the quarks may be obtained from those of the leptons by setting the Major ana mass parameters to zero, their coeffcients and mass conditions may also be obtained from those of the leptons in this limit, and the mixing sectors are analogous

If no Majorana masses are incorporated, the system of boundary equations yields the following mass conditions,

$$
\begin{gathered}
S_{c_{3}}^{-5}=0 \\
S_{c_{2}}^{-3}=0 \\
S_{c_{1}}^{+} S_{c_{3}}^{-}+M_{2}^{2} S_{c_{1}}^{+} S_{c_{3}}^{-}=0 \\
2 S_{c_{3}}^{+}\left(M_{2}^{2} S_{c_{3}}^{-} S_{c_{1}}^{-}+S_{c_{1}}^{-} S_{c_{3}}^{-}\right)-(k z)^{4} M_{2}^{2} S_{c_{1}}^{-} \sin ^{2} \theta_{G}=0 \\
+\left[M_{1}^{2} S_{c_{1}}^{+} S_{c_{2}}^{-} S_{c_{2}}^{+}\left(M_{2}^{2} S_{c_{3}}^{-} S_{c_{1}}^{+}+S_{c_{3}}^{-} S_{c_{2}}^{-}+M_{1}^{-} S_{c_{3}}^{-}\right)+S_{c_{2}}^{+} S_{c_{2}}^{-}\left(M_{2}^{2} S_{c_{1}}^{-} S_{c_{1}}^{+} S_{c_{3}}^{-}+S_{c_{1}}^{-} S_{c_{1}}^{+} S_{c_{3}}^{-} S_{c_{1}}^{-}+2 S_{c_{1}}^{+} S_{c_{1}}^{-} S_{c_{3}}^{-}-S_{c_{2}}^{+} S_{c_{2}}^{-} S_{c_{3}}^{-}\right)\right](k z)^{4} \sin ^{2} \theta_{G} \\
-M_{1}^{2} S_{c_{3}}^{-}(k z)^{8} \sin ^{4} \theta_{G}=0
\end{gathered}
$$

where $c_{1}, c_{2}$, and $c_{3}$ correspond to the parameter values of the respective multiplets and we have dropped the dependence on $m_{n}, z=L_{1}$ and $v$

The first three conditions correspond to non-SM fermons, whle the fourth and fifth correspond to down-type and up-type fermions respectively In total there are four mass conditions for the charge $1(5 / 3)$ leptons (quarks), three for the charge $-1(-1 / 3)$ sector and five for charge $0(2 / 3)$ sector Both the upper and lower charged sectors have an unmixed state, resulting in five groups of equations to be solved for 12 mass conditions and coefficient sets These five sectors are summarized in Tables 35 and 36

In a similar fashion to the gauge bosons, the full fermion profile $F_{\ell}^{f}$ associated with a given solution set $f$ is the superposition of all of the $f_{\ell_{\imath}}\left(m_{n}, z, v\right), \imath=1,2,3$, 
Table 3.5: Sectors of quarks mixed by EWSB through the Higgs vev $v$. Recall that $t_{1_{L}}, b_{1_{L}}$ and $\hat{t}_{2_{R}}, B_{3_{R}}^{\prime}$ are the left- and right-handed SM quarks.

\begin{tabular}{|c||c|c|c|c|c|}
\hline Group & 1 & 2 & 3 & 4 & 5 \\
\hline Mixed Quark & $\chi_{1}, \chi_{3}, \Xi_{3}, \Xi_{3}^{\prime}$ & $\chi_{2}$ & $b_{1}, b_{3}, B_{3}, B_{3}^{\prime}$ & $b_{2}$ & $\begin{array}{c}\tilde{t}_{1}, t_{1}, \hat{t}_{1}, \tilde{t}_{2}, t_{2}, \\
\hat{t}_{2}, \tilde{t}_{3}, t_{3}, T_{3}, T_{3}^{\prime}\end{array}$ \\
\hline $\begin{array}{c}\text { Combined } \\
\text { Quark }\end{array}$ & $\chi_{1}, \chi_{3}$ & - & $b_{1}, b_{3}, B_{3}^{\prime}$ & - & $\left(\tilde{t}_{1}, \tilde{t}_{3}\right)$, \\
Independent & $\Xi_{3}, \Xi_{3}^{\prime}$ & - & $B_{3}$, & - & $\left.\tilde{t}_{2}, T_{3}, T_{3}^{\prime}, \hat{t}_{2}, t_{3}\right)$ \\
\hline Quark & - & $\chi_{2}$ & - & $b_{2}$ & - \\
\hline Unmixed & & & & & \\
\hline
\end{tabular}

evaluated with the specified coefficients. ${ }^{7}$ As such, the $4 \mathrm{D}$ coupling between a gauge boson $G$ and two fermions $f_{1}$ and $f_{2}$ has the form

$$
g_{G \bar{f}_{1} f_{2}}=g_{5} \int_{L_{0}}^{L_{1}} \frac{d z}{(k z)^{4}} \sum_{\alpha, 7} f_{\ell_{2}}^{f_{1} \dagger}\left(m_{n}, z ; v\right) T^{\alpha} f_{\alpha}^{G}\left(m_{n}, z ; v\right) f_{\ell_{\imath}}^{f_{2}}\left(m_{n}, z ; v\right)
$$

and the profile normalization condition is

$$
\int_{L_{0}}^{L_{1}} \frac{d z}{(k z)^{4}} \sum_{\imath} f_{\ell_{\imath}}^{f \dagger}\left(m_{n}, z ; v\right) f_{\ell_{\imath}}^{f}\left(m_{n}, z ; v\right)=1
$$

where $i$ and $f$ are defined as before. The generators for the 5 and 10 representations are given explicitely in Appendix B. It can be shown using the boundary conditions

\footnotetext{
${ }^{7}$ Any coefficients in the multiplet which were not a part of the solution set are taken to be zero
} 
Table 3.6: Sectors of leptons mixed by EWSB through the Higgs vev $v$. Recall that $n_{1_{L}}, \ell_{1_{L}}$ and $\hat{n}_{2_{R}}, L_{3_{R}}^{\prime}$ are the left- and right-handed SM leptons.

\begin{tabular}{|c|c|c|c|c|c|}
\hline Group & 1 & 2 & 3 & 4 & 5 \\
\hline Mixed Lepton & $\begin{array}{c}\kappa_{1}, \kappa_{3}, K_{3}, \\
K_{3}^{\prime}\end{array}$ & $\kappa_{2}$ & $\ell_{1}, \ell_{3}, E_{3}, E_{3}^{\prime}$ & $\ell_{2}$ & $\begin{array}{c}\tilde{\eta}_{1}, \eta_{1}, \hat{\eta}_{1}, \tilde{\eta}_{2}, \eta_{2} \\
\hat{\eta}_{2}, \tilde{\eta}_{3}, \eta_{3}, N_{3} \\
N_{3}^{\prime}\end{array}$ \\
\hline $\begin{array}{l}\text { Combined } \\
\text { Lepton }\end{array}$ & $\kappa_{1}, \kappa_{3}$ & - & $\ell_{1}, \ell_{3}, E_{3}^{\prime}$ & - & $\begin{array}{c}\left(\tilde{\eta}_{1}, \tilde{\eta}_{3}\right) \\
\left(\eta_{1}, \hat{\eta}_{1}, \eta_{2}, \hat{\eta}_{2}, \eta_{3}\right)\end{array}$ \\
\hline $\begin{array}{c}\text { Independent } \\
\text { Lepton }\end{array}$ & $K_{3}, K_{3}^{\prime}$ & - & $E_{3}$, & - & $\tilde{\ell}_{2}, N_{3}, N_{3}^{\prime}$ \\
\hline $\begin{array}{l}\text { Unmixed } \\
\text { Lepton }\end{array}$ & - & $\kappa_{2}$ & - & $\ell_{2}$ & - \\
\hline
\end{tabular}

that the normalization coefficient for a given fermion will also normalize its chiral partner (when evaluated with the appropriate mass) [50].

\subsubsection{The Coleman-Weinberg potential}

As previously discussed, the Higgs boson in the MCHM arises as the zero mode of the $A_{5}^{\hat{4}}$ scalar. Note that although this profile is linear in the warped coordinate $z$, in the flat-space coordinate $y$ the profile is extremely pcaked toward the TcV brane. Because the Higgs boson arises from the gauge sector in the MCHM, it does not have a potential at leading order as the SM Higgs does. Instead, the Higgs potential arises 
primarily from loop contributions of the heavy gauge bosons and the top and bottom quarks. $^{8}$ These one-loop interactions are the same effects that lead to the Planck scale corrections to the Higgs mass in the Standard Model.

The possibility that radiative corrections can induce spontaneous symmetry breaking - i.e., that the Higgs can gain a potential from loop corrections without having one at tree level - was first proposed by S. Coleman and E. Weinberg in 1973. To first order, the Higgs potential is given by their Coleman-Weinberg potential. In $4 \mathrm{D}$ theories, this has the following form [57]

$$
V_{C W}^{(4 D)}(\phi)=V_{0}(\phi)+i \sum_{r} \int_{0}^{\infty} \frac{d^{4} k}{(2 \pi)^{4}} \sum_{n=1}^{\infty} \frac{1}{2 n}\left(\frac{\frac{1}{2} g_{r} \phi^{2}}{k^{2}-i \epsilon}\right)^{n}+\text { counterterms }
$$

where $V_{0}$ is the tree-level potential. The index $r$ sums over all particles that obtain mass through the Higgs mechanism by coupling to the Higgs with strength $g_{r}$. The integral term is obtained by summing all one-loop diagrams whose external legs are the scalars $\phi$. By using the series sum

$$
-\ln (1-z)=\sum_{k=1}^{\infty} \frac{z^{k}}{k}
$$

and Wick rotating $\left(k^{2} \rightarrow-k^{2}, k_{0} \rightarrow i k_{0}\right)$, we obtain (taking $\epsilon \rightarrow 0$ )

$$
V_{C W}^{(4 D)}(\phi)=V_{0}(\phi)+\frac{1}{2} \sum_{r} \int \frac{d^{4} k}{(2 \pi)^{4}} \ln \left(1+\frac{g_{r} \phi^{2}}{2 k^{2}}\right)+\text { counterterms }
$$

Note that if the argument of the logarithm is set to zero and evaluated at the vev, $\phi=v$, it is equivalent to the mass condition for the propagating particle,

$$
k^{2}+\frac{1}{2} g_{r} v^{2}=-m^{2}+\frac{1}{2} g_{r} v^{2}=0 \rightarrow m^{2}=\frac{1}{2} g_{r} v^{2}
$$

In 5D Kaluza-Klein theories, the Coleman-Weinberg potential - with $\phi$ re-

\footnotetext{
${ }^{8}$ We neglect the contributions of the light fermions in comparison with these.
} 
placed by the vev $v$ - is similarly given by $[53,4]$

$$
\begin{aligned}
V_{C W}(v)= & \sum_{r} \pm \frac{N_{r}}{(4 \pi)^{2}} \int d p p^{3} \ln \left(\rho_{r}\left(-p^{2}\right)\right), \\
=\frac{3}{(4 \pi)^{2}} \int_{0}^{\infty} d p p^{3}[ & 6 \ln \left(\rho_{W}\left(-p^{2}\right)\right)+3 \ln \left(\rho_{Z}\left(-p^{2}\right)\right) \\
& \left.-4\left(\ln \left(\rho_{t}\left(-p^{2}\right)\right)+\ln \left(\rho_{b}\left(-p^{2}\right)\right)\right)\right],
\end{aligned}
$$

where $N_{r}$ is the number of degrees of freedom, and the plus applies to bosons while the minus applies to fermions. This form is analogous to that of Eq. (3.55); $V_{0}=0$ as there is no tree-level potential, counterterms are unneeded due to the convergence of the loop integrands, and the 4D mass condition is replaced by the analogous spectral functions, $\rho$. These are simply the particle mass conditions of the $W$ boson, $Z$ boson, bottom quark and top quark where the term independent of $\sin \theta_{G}$ has been divided out,

$$
\begin{gathered}
\rho_{\imath}\left(m^{2}\right)=1+F_{\imath}\left(m^{2}\right) \sin \theta_{G}\left(L_{1}, v\right)^{2}, \quad i=W, Z, b \\
\rho_{t}\left(m^{2}\right)=1+\frac{F_{t 1}\left(m^{2}\right)}{2 F_{d}\left(m^{2}\right)}(m) \sin \theta_{G}\left(L_{1}, v\right)^{2}+\frac{F_{t 2}\left(m^{2}\right)}{2 F_{d}\left(m^{2}\right)}(m) \sin \theta_{G}\left(L_{1}, v\right)^{4}
\end{gathered}
$$

where the form-factors $F_{\imath}(m)$ are given by

$$
\begin{aligned}
& F_{Z}\left(m^{2}\right)=\sec ^{2} \theta_{w} F_{W}(m)=\frac{m_{n} k L_{1} \sec ^{2} \theta_{w}}{2 C_{A}^{\prime}\left(m_{n}, L_{1}\right) S_{A}\left(m_{n}, L_{1}\right)} \\
& F_{b}\left(m^{2}\right)=-\frac{M_{2}^{2} \dot{S}_{c_{1}}^{-}(k z)^{4}}{2 S_{c_{3}}^{+}\left(M_{2}^{2} S_{c_{3}}^{-} \dot{S}_{c_{1}}^{-}+S_{c_{1}}^{-} \dot{S}_{c_{3}}^{-}\right)} \\
& F_{d}\left(m^{2}\right)=M_{1}^{2} S_{c_{1}}^{+} \dot{S}_{c_{2}}^{-} \dot{S}_{c_{2}}^{+}\left(M_{2}^{2} S_{c_{3}}^{-} \dot{S}_{c_{1}}^{-}+S_{c_{1}}^{-} \dot{S}_{c_{3}}^{-}\right)+S_{c_{2}}^{+} \dot{S}_{c_{2}}^{-}\left(M_{2}^{2} \dot{S}_{c_{1}}^{-} \dot{S}_{c_{1}}^{+} S_{c_{3}}^{-}+S_{c_{1}}^{-} \dot{S}_{c_{1}}^{+} \dot{S}_{c_{3}}^{-}\right) \\
& F_{t 1}\left(m^{2}\right)=M_{2}^{2} S_{c_{2}}^{+} S_{c_{3}}^{-} \dot{S}_{c_{2}}^{-}+M_{1}^{2}\left(2 M_{2}^{2} S_{c_{1}}^{+} S_{c_{3}}^{-} \dot{S}_{c_{1}}^{-}+2 S_{c_{1}}^{+} S_{c_{1}}^{-} \dot{S}_{c_{3}}^{-}-\dot{S}_{c_{2}}^{+} \dot{S}_{c_{2}}^{-} \dot{S}_{c_{3}}^{-}\right) \\
& F_{t 2}\left(m^{2}\right)=-M_{1}^{2} \dot{S}_{c_{3}}^{-}(k z)^{8}
\end{aligned}
$$

where the mass dependence in the $S_{c}\left(m, L_{1} ; v\right)$ has been omitted. 
Similarly to the SM case, the Higgs vacuum expectation value is determined by minimizing the Coleman-Weinberg potential, while its mass is determined by evaluating the second derivative at the vev [4]. As such, if the Coleman-Weinberg potential is divergent, so is the Higgs mass. This is the origin of the Hierarchy problem.

However, the 5D potential of Eq. (3 59) is finite and calculable. Fig (3.3) plots the integrand of the CW potential for a particular parameter set; one can see that the integrand is exponentially suppressed as the momentum increases, creating an effective cut-off of the integration at an appropriate scale, eliminating the need for counterterms and avoiding the Hierarchy problem.

This exponential suppression can also be demonstrated analytically by using the asymptotic properties of Bessel functions (see Appendix A). Consider for example the $Z$ form-factor $F_{Z}\left(m^{2}\right)$, which is inversely proportional to $C_{A}^{\prime}\left(m_{n}, L_{1}\right) S_{A}\left(m_{n}, L_{1}\right)$. Assuming that $p L_{1} \gg 1, p L_{0} \ll 1$, and Eqs. (A.9-A.13), one can show that

$$
\begin{aligned}
& C_{A}^{\prime}\left(m_{n}, L_{1}\right)=\frac{1}{2} \pi m^{2} z\left(Y_{0}\left(m L_{0}\right) J_{0}\left(m L_{1}\right)-J_{0}\left(m L_{0}\right) Y_{0}\left(m L_{1}\right)\right) \\
& \stackrel{\text { Wick rot }}{\rightarrow} \frac{1}{2} \pi \imath m^{2} z\left(-\frac{2}{\pi} K_{0}\left(m L_{0}\right) I_{0}\left(m L_{1}\right)+I_{0}\left(m L_{0}\right) \frac{2}{\pi} K_{0}\left(m L_{1}\right)\right) \\
& \quad \approx \imath m^{2} z\left(\frac{\exp \left(m L_{1}\right)}{\sqrt{2 \pi m L_{1}}}\left(\ln \left(\frac{m L_{0}}{2}\right)+\gamma\right)+\sqrt{\frac{\pi}{2 m L_{1}}} \exp \left(-m L_{1}\right)\right) .
\end{aligned}
$$

Note that the first term is exponentially enhanced, while the second is exponentially suppressed. As such, the first term dominates and $C_{A}^{\prime}\left(m_{n}, L_{1}\right)$ is approximately proportional to $\exp \left(m L_{1}\right) . S_{A}\left(m_{n}, L_{1}\right)$ can be shown to have a similar exponential proportionality, and thus the form factor $F_{Z}\left(m^{2}\right)$ is doubly exponentially suppressed. Furthermore, considering that $F_{Z}\left(m^{2}\right)$ is small, we may make use of the Taylor expansion

$$
\ln (1+x)=x-\frac{1}{2} x^{2}+\frac{1}{3} x^{3} \ldots
$$

to show that the Coleman-Weinberg integrand will be proportional to $F_{Z}\left(m^{2}\right)$, and 


\section{Suppression in the integrand of the CW potential}

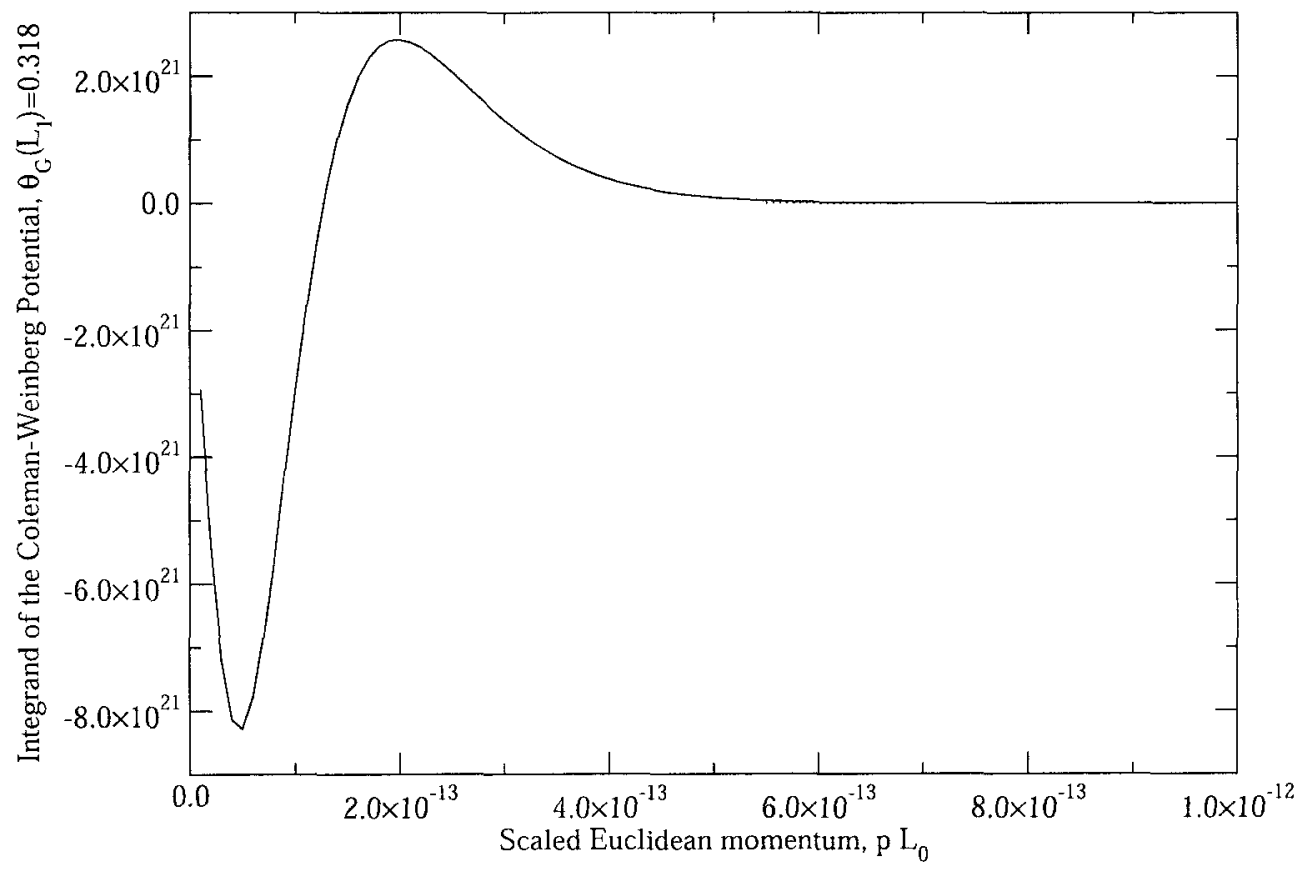

Figure 3.3: The integrand of the Coleman-Weinberg potential for $k L=30, c_{1}=0.24$, $c_{2}=-0.41, c_{3}=0.58, M_{1}=2.3$, and $M_{2}=0.4$; these values result in a Higgs vev corresponding to $\theta_{G}\left(L_{1}\right)=0.318$.

therefore exponentially suppressed as well.

\subsubsection{Parameters of the Minimal Composite Higgs Model}

The Minimal Composite Higgs model depends on parameters $g, \theta_{w}, M_{z}^{(0)}, k L=$ $\ln \left(k L_{1}\right)$, and the various $c_{2}$ and $M_{2}$ that are necessary to specify the fermion multiplets. The first three of these are defined to be the usual SM parameters: $g=0.649$, $\theta_{w}=28.75^{\circ}, M_{z}^{(0)}=91.18762 \mathrm{GeV}$. The fourth parameter is usually taken to be 
$k L \approx 30[4]$

Once the fermion parameters of the third quark generation have been chosen, the Coleman-Weinberg potential may be minimized to determine the value of $\theta_{G}\left(L_{1}\right)$ [4]. ${ }^{9}$ Using this value, $k$ is determined by solving the $Z$ boson mass condition such that $m=M_{Z}^{(0)}$. Having obtained $k, L_{0}$ and $L_{1}$ are given by their original definitions, $L_{0}=1 / k$ and $L_{1}=\exp (k L) / k$.

At this point the vev can be obtained by rearranging the equation for $\theta_{G}\left(L_{1}\right)$,

$$
v=\frac{2 \theta_{G}\left(L_{1}\right)}{g_{5} \sqrt{k\left(L_{1}^{2}-L_{0}^{2}\right)}},
$$

and, finally, the Higgs mass can be determined by evaluating the second derivative of the Coleman-Weinberg potential at the vev, $m_{H}=V_{C W}^{\prime \prime}(v)$, as previously discussed [4].

In an ideal world one would like to choose a set of parameters such that the resulting Higgs vev is that of the SM. However, in this parametrization scheme there is a close relationship between the Higgs vev $v$ and the upper bound on the fifth dimension $L_{1}$. This relationship is plotted in Fig. (3.4) for $k L=30$; one can see that as the Higgs vev approaches the SM value of $246 \mathrm{GeV}$, the length of the fifth dimension approaches zero. As such, we cannot retain the SM Higgs vev value; we must strike a balance between keeping the vev value close to that of the SM and keeping $L_{1}$ near $1 \mathrm{TeV}$.

The choice of parameters has been further constrained by electroweak precision tests in the literature. In the MCHM with the MSW fermion embedding, if the light

\footnotetext{
${ }^{9}$ Note that the minimization condition can be written entirely in terms of the initial parameters and $\theta_{G}\left(L_{1}\right)$ - independently of $k$-by transforming the integration variable to $p L_{0}$.
} 


\section{The relation between $L_{1}$ and the Higgs vev}

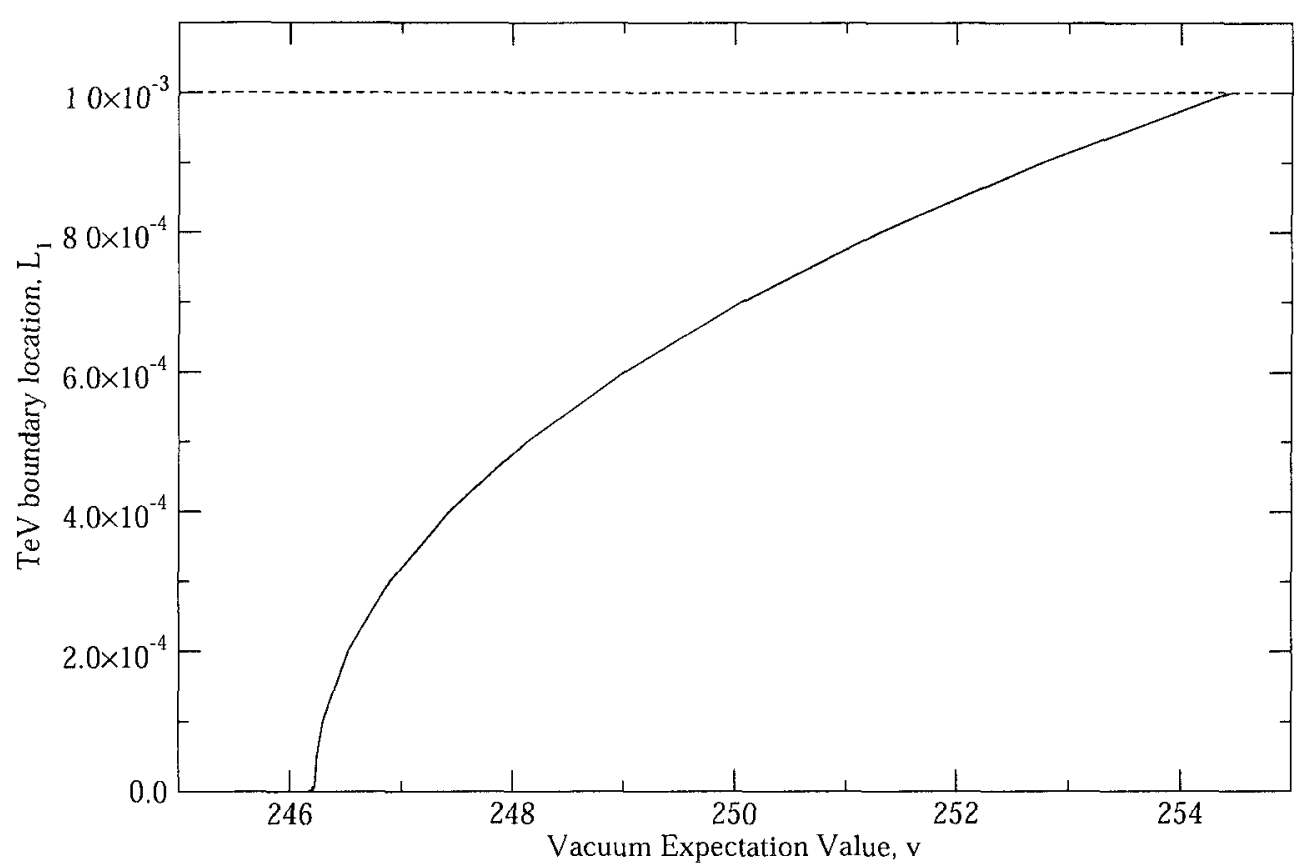

Figure 3.4: A plot of the relationship between the Higgs vev $v$ and $L_{1}$ for $k L=30$. The dotted lines indicate the ideal values of $v=246 \mathrm{GeV}$ and $L_{1}=1 / \mathrm{TeV}$.

fermions are placed close to the Planck brane - as is required to obtain realistic masses - then the KK mass scale $M_{k k}=\frac{1}{L_{1}}>1.4 \mathrm{TeV}$, implying that $L_{1}<0.714$ $\mathrm{TeV}^{-1}[49,54]$. The parameters of the third quark generation have further been constrained to the following regions of parameter space: $0 \leq\left|c_{1}\right| \leq 0.3,0.35 \leq\left|c_{2}\right| \leq$ $0.45,0.55 \leq\left|c_{3}\right| \leq 0.6, M_{1} \geq 1$, and $M_{2}<M_{1}$; ranges such that $c_{1}>0$ and $c_{2}<-0.4$ are favoured by electroweak precision constraints (EWPC) $[4,50]$. Furthermore, while light fermions generally require $c$ values higher than 0.5 to obtain the right masses, EWPC require that $c<0.75[52]$.

In all of the following work, we have used the initial parameters listed Table 3.7. 
Table 37 Inıtıal parameters used to solve the Coleman-Weinberg potential, as well as the resultıng $k, v, L_{0}$ and $L_{1}$ The parameters with superscript $q_{3}$ refer to those of the third quark generation, whle $c_{\text {light }}$ applies to all other fermions

\begin{tabular}{|c|c|c|c|c|c|c|c|c|c|}
\hline$k L$ & $c_{1}^{\left(q_{3}\right)}$ & $c_{2}^{\left(q_{3}\right)}$ & $c_{3}^{\left(q_{3}\right)}$ & $c_{\text {light }}$ & $M_{1}^{\left(q_{3}\right)}$ & $M_{1}^{\left(q_{3}\right)}$ & $k[\mathrm{GeV}]$ & $v[\mathrm{GeV}]$ & $L_{1}\left[\mathrm{GeV}^{-1}\right]$ \\
\hline 30 & 024 & -041 & -058 & 070 & 23 & 05 & $1497 \quad 10^{16}$ & 250218 & $714010^{-4}$ \\
\hline
\end{tabular}

They are convenient as they abide by the constraints above, but result in a KK mass scale just above $14 \mathrm{TeV}$, so that the KK masses are as small as possible Furthermore, parts of this parameter set was used for other purposes in the literature [50], which allowed for cross-checks of our work

Table 38 Table of $Z, X$, and $A^{3}$ boson mass both before and after EWSB

\begin{tabular}{|c|c|c|c|c|c|c|}
\hline \multirow{2}{*}{ KK order } & \multicolumn{3}{|c|}{ Mass Before EWSB } & \multicolumn{3}{c|}{ Mass After EWSB } \\
& $Z[\mathrm{TeV}]$ & $X[\mathrm{TeV}]$ & $A^{3}[\mathrm{TeV}]$ & $Z[\mathrm{TeV}]$ & $X[\mathrm{TeV}]$ & $A^{3}[\mathrm{TeV}]$ \\
\hline \hline 0 & 0 & & - & 9118 & - & \\
\hline 1 & 3442 & 3368 & 5367 & 3437 & 3368 & 5372 \\
\hline 2 & 7809 & 7732 & 9826 & 7804 & 7732 & 9831 \\
\hline 3 & 12199 & 12121 & 14249 & 12194 & 12121 & 14254 \\
\hline 4 & 16595 & 16515 & 18661 & 16590 & 16515 & 18667 \\
\hline
\end{tabular}

As we will be neglecting zero-mode fermion masses, for simplicity we will not distinguish between the different light fermons, we will use the same parameters for 
all leptons, neutrinos, and the first two quark generations However, it should be noted that different chorces of parameters will yield extremely different spectra of KK fermions

For future reference, the parameters in Table 37 yleld the spectrum of masses for the $X, Z$ and $A^{3}$ bosons found in Table 38 As expected, the KK masses begin at roughly $25 M_{k k}=25 \times 14 \mathrm{TeV}=35 \mathrm{TeV}$ In accordance with Fig 32, the $X$ boson masses are left unchanged by the particle mixing The $Z^{(0)}$ mass is shifted up to its SM value, whlle the $Z \mathrm{KK}$ masses are shifted down (but remain larger than the $X$ masses) Similarly, the $A^{3}$ masses are shifted slightly upward 


\section{Chapter 4}

\section{The Higgsstrahlung cross section}

\subsection{Higgsstrahlung as a probe of compositeness}

In the Higgsstrahlung interaction an electron-positron collision produces a SM $Z$ boson and a Higgs (see Fig. 4.1).

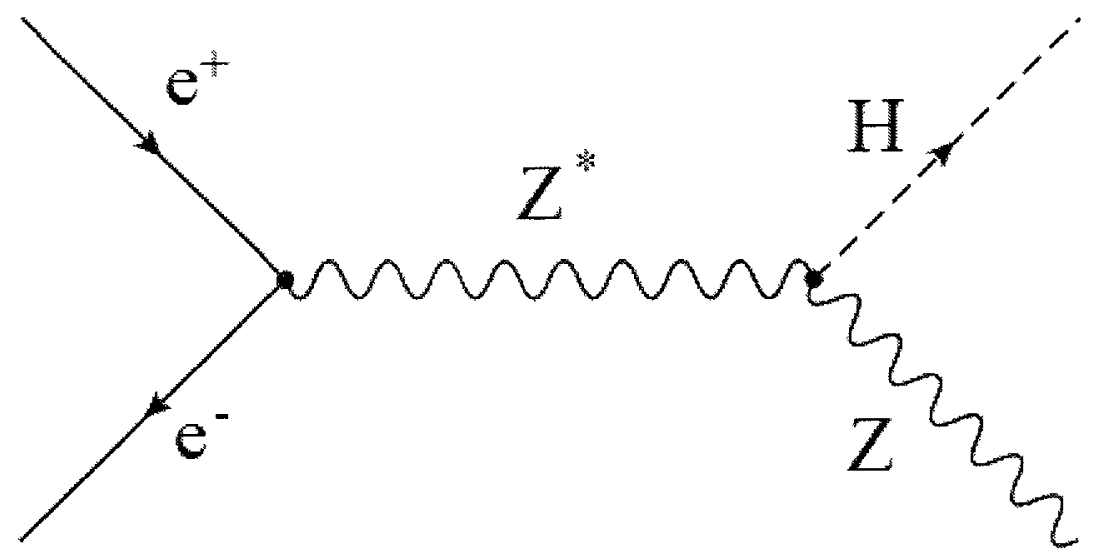

Figure 4.1: The Higgsstrahlung interaction, $e^{+} e^{-} \rightarrow Z^{0} H$. 
We have chosen to study this interaction because the Higgs coupling has a similar structure to that of the $Z^{(n)}$ loop diagram that provides a correction to the Higgs mass In the Standard Model this loop diagram is divergent, and contributes to the Hierarchy problem However, it is not divergent in the MCHM, due to the exponential suppression in the integrand of the Coleman-Weinberg potential, as we saw in the previous chapter In the effective 4D KK picture this is due to the warped geometry of the 5D model, in terms of the CFT picture it is duc to the finite size of the composite Higgs boson

Under either interpretation, one would expect the Higgsstrahlung cross section to display a similar suppression The 5D structure of the Higgsstrahlung $Z^{(n)} Z^{(0)} H$ interaction is very similar to that of the $Z^{(n)} Z^{(n)} H H$ interaction, the only difference being the replacement of a $n^{\text {th }}$ order $Z$ boson profile with a zeroth order profile Furthermore, one would expect the finite size of the composite Higgs to have the same effect on the Higgsstrahlung interaction as on the loop diagram

Another attractive feature of Higgsstralung is that it is an s-channel interaction therefore all of the initial energy from the fermions is transmitted directly through the propagator into the Higgs vertex This allows for a clean high-energy interaction

The unpolarized Standard Model cross section for this interaction is given by

$$
\sigma_{\mathrm{SM}}=\frac{G_{f}^{2} M_{Z}^{(0)}}{24 \pi s^{2}} \lambda^{\frac{1}{2}}\left(s, M_{Z}^{(0) 2}, M_{H}^{2}\right)\left(C_{V}^{2}+C_{A}^{2}\right) \frac{\left[12 M_{Z}^{(0) 2} s+\lambda\left(s, M_{Z}^{(0) 2}, M_{H}^{2}\right)\right]}{\left(s-M_{Z}^{(0) 2}\right)^{2}+\Gamma_{Z}^{(n) 2} M_{Z}^{(0) 2}},
$$

where $G_{f}$ is the Fermi coupling constant, and

$$
\lambda(x, y, z)=x^{2}+y^{2}+z^{2}-2 x y-2 x z-2 y z
$$

Note that when $s \gg M_{z}^{(0) 2}, \lambda\left(s, M_{Z}^{(0) 2}, M_{H}^{2}\right) \approx s^{2}$ and therefore the cross section falls like $s^{-1}$ We will compare our MCHM Higgsstrahlung cross sections to Eq (4 1), we 
expect that the MCHM cross section will be strongly suppressed in comparison to the SM cross section above the KK mass scale

As the outgoing Higgs and $Z^{(0)}$ bosons are not charged, and the $A_{\mu}^{a}$ bosons do not couple to electron anti-electron pars, the only bosons that can propagate through this interaction before mixing are the $Z_{\mu}^{(n)}$ and the $X_{\mu}^{(n)}$

\subsection{Two equivalent perspectives}

We have approached the calculation of the MCHM Higgsstrahlung cross section from two different but equvalent perspectives Both involve integrating over the fifth dimension to obtain a four-dimensional effectıve theory

In the first case, we integrate the 5D Lagrangian before deriving Feynman rules As a result, we obtain the usual 4D Feynman propagator for a vector boson However, our 4D vertex rules now include an additional factor the integral over $z$ of the $z$-dependent 5D vertex components and the profiles of the relevant particles In this case, we are treating our propagating bosons as KK modes To obtain the full cross section, we would have to sum over all of the infinite number of KK modes, which is obviously not feasible However, by truncating the sum we can obtain an approximate cross section Despite the approximation, this approach has several advantages, notably that it is straightforward to include the zero-mode masses, gauge boson widths, and the effects of particle mixing in the calculation We will refer to this approach as Effective Vertex Approach (EVA)

In the second case, we assemble the interaction amplitude using 5D Feynman rules, and then integrate over the fifth dimension This method has the advantage of 
including all of the propagating KK modes automatically, however, the inclusion of zero-mode masses, widths, and mixing effects becomes difficult We will refer to this approach as Effective Cross-section Approach (ECA)

\subsection{A first approximation: Higgsstrahlung without particle mixing}

As a first approach to the analysis, we make an approximation in that we neglect partıcle mixing due to electroweak symmetry breakıng As discussed in Sec 32 , we expect the extra contributions to the $Z$ from the $X$ and $A^{3}$ bosons to be quite small, as the $Z^{(n)}$ boson after EWSB should still predominantly correspond to the original $Z^{(n)}$ boson, the same ratıonale applies to the mixıng in the electron states Therefore we expect this to be an acceptable approximation for our cross section

Furthermore, we also neglect the effects of non-SM fermions, KK mode fermions channels are not included in any boson decay widths ${ }^{1}$

\subsubsection{Feynman rules}

A comprehensive summary of the 5D Feynman rules of the model - in the absence of mixed particle states can be found in Appendix C However, we will summarıze those relevant to the cross section here

In the EVA view each gauge KK mode is associated with the usual Feynman propagator for a heavy gauge boson However, in the ECA view the 5D $Z$ and $X$

\footnotetext{
${ }^{1}$ The KK fermion channels in the gauge boson decay widths will be addressed in Sec 325 when we compute the cross section with full particle mixing
} 
boson propopagators from $z$ to $z^{\prime}$ in untary gauge are given by [38]

$$
-\imath G\left(z, z^{\prime}, p\right)\left(\eta^{\mu \nu}-\frac{p^{\mu} p^{\nu}}{p^{2}}\right)-\imath G\left(z, z^{\prime}, 0\right)\left(\frac{p^{\mu} p^{\nu}}{p^{2}}\right)
$$

The function $G\left(z, z^{\prime}, p\right)$ is the Green's function of the equations of motion, Eq (3 17) As such it is defined by

$$
\left[p^{2}-\frac{1}{z} \partial_{z}+\partial_{z}^{2}\right] G\left(z, z^{\prime}, p\right)=k z \delta\left(z-z^{\prime}\right),
$$

where we have Fourier transformed the first four spatial coordinates $x_{\mu}$ to momentum space coordinates $p_{\mu}[51,38]$ The coordinate of the fifth dimension $z$ has been left as a spatial coordinate This differential equation has the general solution

$$
G(u, v, p)=\frac{\pi}{2} \frac{k u v}{A D-B C}\left[A J_{1}(p u)+B Y_{1}(p u)\right]\left[C J_{1}(p v)+D Y_{1}(p v)\right]
$$

where $u=\min \left(z, z^{\prime}\right), v=\max \left(z, z^{\prime}\right)$, the coefficients $A, B, C, D$ are determined by the appropriate BCs for the particle in question, and $J$ and $Y$ are the Bessel functions The $Z$ boson propagator will have $A=-Y_{0}\left(p L_{0}\right), B=J_{0}\left(p L_{0}\right), C=-Y_{0}\left(p L_{1}\right)$, and $D=J_{0}\left(p L_{1}\right)$, which correspond to Neumann-Neumann BCs The $X$ will have $A=-Y_{1}\left(p L_{0}\right), B=J_{1}\left(p L_{0}\right), C=-Y_{0}\left(p L_{1}\right)$, and $D=J_{0}\left(p L_{1}\right)$, which correspond to Dirıchlet-Neumann BCs

We also have the following vertices, which can be obtained by taking the functional derivative of the coupling terms in the model Lagrangian,

$$
\begin{gathered}
G_{\mu}^{(n)} Z_{\mu}^{(m)} H \rightarrow \frac{\imath g_{z} g_{G}}{2 k z} \frac{g_{5}}{g} \eta^{\mu \nu} v f_{H}(z)^{2} f_{G}^{(n)}(z) f_{Z}^{(n)}(z) \\
\bar{\Psi}_{L R} G_{\mu}^{(n)} \Psi_{L R} \rightarrow \frac{g_{G}}{2(k z)^{4}} \frac{g_{5}}{g} C_{f G}^{L R} \gamma_{\mu} f_{G}^{(n)}(z) f_{f}(z)^{2}
\end{gathered}
$$

where $G=Z, X$ are $4 \mathrm{D}$ gauge KK modes, $H$ is the $4 \mathrm{D}$ Higgs boson, $f_{f}(z), f_{G}(z)$ and $f_{H}(z)$ denote the appropriate fermion, gauge boson and Higgs profiles, given by 
Eqs (3 18-3 21), Eqs (3 30-3 33) and Eq (3 22) in the non-mixing case We have defined the following constants,

$$
\begin{gathered}
C_{f Z}^{L R}=T^{3_{L}}-Q \sin ^{2} \theta_{w}, \quad C_{f \lambda}^{L R}=\frac{\left(Q-T^{3_{L}}\right) \sin ^{2} \theta_{w}-T^{3_{R}} \cos ^{2} \theta_{w}}{\cos 2 \theta_{w}} \\
g_{z}=\frac{g}{\cos \theta_{w}}, \quad g_{x}=\frac{\sqrt{\cos 2 \theta_{w}}}{\cos \theta_{w}}, \quad g_{5}=g \sqrt{\frac{\ln \left(k L_{1}\right)}{k}}
\end{gathered}
$$

Here $T^{3_{L R}}$ denotes the appropriate generator element for the right- or left-handed fermion in question Note that the first coupling of $\mathrm{Eq}$ (46) originates from a $G_{\mu}^{(n)} Z_{\mu}^{(m)} H H$ coupling where a single Higgs has been replaced with the vev, $H \rightarrow v$ This is why there are two factors of the Higgs profile, this coupling ultimately originates from the quartic gauge coupling in the gauge Lagrangian of Eq (38) The couplings of 5D particles would have the same form except that the profiles would be absent as they are included in the full 5D boson

It should also be noted that the left- and right handed fermon couplings have the same algebraic form due to the fact that the coupling terms in the fermion Lagrangıan are the same for both chiralities However, due to the multiplet structure of the fermion sector, the values of $T^{3_{L} R}$ corresponding to our right- and left-handed SM fermions will be different, although the algebraic form is the same for all feimions the coupling values are not

\subsubsection{Cross sections in the Effective Vertex Approach}

Using the Effectıve Vertex Approach, the 4D effective amplitudes corresponding to the Higgsstrahlung cross section for generıc fermions are

$$
M_{G^{(n)}}^{L R}=-\frac{g_{z} g_{G}^{2} v}{4} \epsilon^{\nu *}\left(p_{4}\right) Z_{f f G}^{(n)} Z_{G Z H}^{(n 0)} C_{f G}^{L R} \frac{\bar{v}\left(p_{1}\right) \gamma_{\nu}\left(1-\gamma_{5}\right) u\left(p_{2}\right)}{q^{2}-M_{G}^{(n) 2}+\imath \Gamma_{G}^{(n)} M_{G}^{(n)}}
$$


where $p_{1}, p_{2}$, and $p_{4}$ are the momenta of the anti-fermion, fermion, and external $\mathrm{Z}^{(0)}$ boson respectively, $q=p_{1}+p_{2}$ is the momentum exchange, and $\Gamma_{G}^{(n)}$ is the total decay width of the particle propagating in the s-channel. Here $G^{(n)}=Z^{(n)}, X^{(n)}$, $L, R$ refer to the polarization of the fermions, the coupling factors $C_{f G}^{L, R}$ are given by Eq. (4.7), and the coefficients $Z_{G f f}^{(n)}(z)$ and $Z_{G Z H}^{(n, 0)}(z)$ are the integrals of the 5dimensional profiles,

$$
\begin{aligned}
Z_{G f f}^{(n)} & =\int_{L_{0}}^{L_{1}} d z \frac{1}{(k z)^{4}} \frac{g_{5}}{g} f_{G}^{(n)}\left(m_{n}, z\right) f_{f}^{(0)}\left(m_{n}, z\right)^{2} . \\
Z_{G Z H}^{(n, 0)} & =\int_{L_{0}}^{L_{1}} d z \frac{1}{k z}\left(\frac{g_{5}}{g}\right)^{2} f_{G}^{(n)}\left(m_{n}, z\right) f_{Z}^{(0)}\left(m_{n}, z\right) f_{H}(z)^{2},
\end{aligned}
$$

We have neglected the fermion masses and made use of the Dirac equation. Note that as we are integrating over the vertex by itself, all of the particles are external and on-shell; therefore the momentum dependence of the propagator profile is evaluated at the appropriate mass $m_{n}$.

In the center-of-mass frame the cross section corresponding to one of these terms is

$$
\begin{aligned}
\sigma_{G^{(n)}}^{L, R}= & \frac{g_{z}^{2} g_{G}^{4} v^{2}}{384 \pi M_{Z}^{(0) 2} s^{2}}\left[12 M_{Z}^{(0) 2} s+\lambda\left(s, M_{Z}^{(0) 2}, M_{H}^{2}\right)\right] \\
& \times \lambda^{\frac{1}{2}}\left(s, M_{Z}^{(0) 2}, M_{H}^{2}\right) \frac{Z_{G f f}^{(n) 2} Z_{G Z H}^{(n, 0) 2}\left(C_{f G}^{L, R}\right)^{2}}{\left(s-M_{G}^{(n) 2}\right)^{2}+\Gamma_{G}^{(n) 2} M_{G}^{(n) 2}}
\end{aligned}
$$

The total cross section for a given polarization is given by

$$
\begin{aligned}
\sigma_{\mathrm{tot}}^{L, R} & =\frac{g_{z}^{2} v^{2}}{384 \pi M_{Z}^{(0) 2} s^{2}}\left[12 M_{Z}^{(0) 2} s+\lambda\left(s, M_{Z}^{(0) 2}, M_{H}^{2}\right)\right] \lambda^{\frac{1}{2}}\left(s, M_{Z}^{(0) 2}, M_{H}^{2}\right) \\
& \times\left[\sum_{n=0}^{\infty} \frac{g_{z}^{2} C_{f Z}^{L, R} Z_{z f f}^{(n)} Z_{Z Z H}^{(n, 0)}}{\left(s-M_{Z}^{(n) 2}\right)^{2}+\Gamma_{Z}^{(n) 2} M_{Z}^{(n) 2}}+\sum_{n=0}^{\infty} \frac{g_{x}^{2} C_{f X}^{L, R} Z_{x f f}^{(n)} Z_{X Z H}^{(n, 0)}}{\left(s-M_{X}^{(n) 2}\right)^{2}+\Gamma_{X}^{(n) 2} M_{X}^{(n) 2}}\right]^{2}
\end{aligned}
$$


The unpolarized cross section $\sigma$ corresponding to Eq. (4.1) can be obtained by adding the unpolarized cross sections and dividing by four,

$$
\sigma_{\text {tot }}=\frac{1}{4}\left(\sigma_{\text {tot }}^{L}+\sigma_{\text {tot }}^{R}\right)
$$

\subsubsection{Cross sections in the Effective Cross-section Approach}

In the ECA approach, to obtain the effective 4D amplitude we integrate the fivedimensional components of the vertices together with the 5D propagator, $G\left(z, z^{\prime} ; q\right)$ of Eq. (4.5). The total effcctive $4 \mathrm{D}$ amplitude is therefore

$$
M_{G}^{L, R}=-\frac{g_{z} v}{4} \epsilon^{* \alpha}\left(p_{4}\right) \bar{v}\left(p_{1}\right) \gamma_{\alpha}\left(1-\gamma_{5}\right) u\left(p_{2}\right) g_{G}^{2} Z_{G}(q, c) C_{f G}^{L, R}
$$

where $G=Z, X$ and $G_{Z}\left(z, z^{\prime} ; q\right)=G^{(N N)}\left(z, z^{\prime} ; q\right)$ and $G_{X}\left(z, z^{\prime} ; q\right)=G^{(D N)}\left(z, z^{\prime} ; q\right)$; the superscripts indicate the appropriate boundary conditions for the boson in question. The parameter $c$ refers to the electron profile parameter. The momenta $p_{1}$, $p_{2}, p_{3}, p_{4}$, and $q$ correspond to the anti-fermion, the fermion, the Higgs, the external $\mathrm{Z}$ boson, and the s-channel boson respectively. The constants $C_{f G}^{L, R}$ are the same as those defined in the previous section (Eq. (4.7)) and $Z_{G}(q, c)$ is the integral of all the 5-dimensional components over $z$ and $z^{\prime}$,

$$
\begin{gathered}
Z_{G}(q, c)=\int_{L_{0}}^{L_{1}} d z \int_{L_{0}}^{z} d z^{\prime}\left(\frac{g_{5}}{g}\right)^{3} \frac{1}{k z^{\prime}} \frac{1}{(k z)^{4}} f_{f}^{(0)}(z)^{2} f_{Z}^{(0)}\left(z^{\prime}\right) f_{H}\left(z^{\prime}\right)^{2} G_{G}\left(z^{\prime}, z ; q\right) \\
+\int_{L_{0}}^{L_{1}} d z \int_{z}^{L_{1}} d z^{\prime}\left(\frac{g_{5}}{g}\right)^{3} \frac{1}{k z^{\prime}} \frac{1}{(k z)^{4}} f_{f}^{(0)}(z)^{2} f_{Z}^{(0)}\left(z^{\prime}\right) f_{H}\left(z^{\prime}\right)^{2} G_{G}\left(z, z^{\prime} ; q\right) .
\end{gathered}
$$

The integration is performed so as to account for the fact that the propagator is in fact a piecewise function: $u=z$ and $v=z^{\prime}$ if $z<z^{\prime}$ while $u=z^{\prime}$ and $v=z$ if $z>z^{\prime}$. Note that in this case the propagator is not an external particle when we perform the integral, and therefore not on-shell; we therefore denote the propagator momentum 
by $q$ and not $m$. For analytic solutions to the integrals over the fifth dimension, please see Appendix E.

We therefore find that the total cross section is

$$
\begin{aligned}
\sigma_{L, R}= & \frac{g_{z}^{2} v^{2}}{384 \pi M_{z}^{(0) 2} s^{2}} \lambda^{\frac{1}{2}}\left(s, M_{z}^{(0) 2}, M_{H}^{2}\right)\left[12 M_{z}^{(0) 2} s+\lambda\left(s, M_{z}^{(0) 2}, M_{H}^{2}\right)\right] \\
& \left.\times\left[g_{z}^{2} Z_{Z}(q, c) C_{f Z}^{L, R}+g_{x}^{2} Z_{X}(q, c) C_{f X}^{L, R}\right)\right]^{2}
\end{aligned}
$$

Let's consider the dimensions of $Z_{G}(q, c)$ for a moment. As each profile $f$ has a dimension of [Energy $]^{\frac{1}{2}}, G\left(z, z^{\prime} ; q\right)$ has dimension [Energy] ${ }^{-1}$ and $g_{5} / g$ has dimension [Energy $]^{-\frac{1}{2}}$, we find that $Z_{G}(q, c)$ has dimension $[\text { Energy }]^{-2}$. Therefore $Z_{G}(q, c)$ has the same dimensons as the SM gauge boson propagator and the $4 \mathrm{D}$ amplitude $M_{G}^{L, R}$ is dimensionless, as it should be for a four body scattering process.

As noted proviously, this approach to the calculation of the cross section is completely equivalent to the EVA approach if propagator widths and zero mode masses are neglected. This can be shown explicitly by means of the following relation which exists between the 4D gauge $\mathrm{KK}$ mode profiles and the 5D propagator [51],

$$
G\left(z, z^{\prime} ; p\right)=\sum_{n=0}^{\infty} \frac{f_{G}^{(n)}(z) f_{G}^{(n)}\left(z^{\prime}\right)}{p^{2}-M_{G}^{(n) 2}}
$$

Using this identity, one finds that the integrals in Eq. (4.15) separate into the two integrals given by Eqs. (4.9) and (4.10), and therefore that

$$
Z_{G}(c, q)=\sum_{n=0}^{\infty} \frac{Z_{G f f}^{(n)} Z_{G Z H}^{(n, 0)}}{q^{2}-M_{G}^{(n) 2}}
$$

Therefore, the cross section obtained in Eq. (4.16) is completely equivalent to that in Eq. (4.12) in the limit that the gauge boson $\mathrm{KK}$ mode widths $\Gamma_{G}^{(n)}$ and zero-mode masses $M_{Z}^{(0)}$ are neglected (as Eq. (4.17) does not include EWSB, and therefore $M_{Z}^{(0)}$ is taken to be zero). 


\subsubsection{Comparison and analysis of cross sections}

Although both the $Z$ and $X$ bosons may propagate in the $e^{+} e^{-} \rightarrow Z^{(0)} H$ cross section, the contribution of the $X$ boson is effectively negligible for our choice of $c=0.7$ for the electron, and remains so unless the electron $c$ parameter becomes quite close to 0.5. As light fermions such as the electron $(c>0.5)$ have profiles peaked toward the Planck brane, their couplings to the $X$ boson - whose profile is zero on the Planck brane due to the Dirichlet $\mathrm{BC}$ - are very weak. Therefore we may neglect the effects of the propagating $X$ boson in the Higgsstrahlung cross section.

Fig. 4.2 compares the $e^{+} e^{-} \rightarrow Z^{(0)} H$ cross section of the SM, the MCHM from ECA, and the MCHM from EVA including the first $6 \mathrm{KK}$ modes. We plot $q^{2} \sigma$ to cancel the $q^{-2}$ falloff of the SM cross section at high $q^{2}$. None of these cross sections include widths, which leads to the resonance spikes at $q=m_{n}$ for the $Z \mathrm{KK}$ modes.

The figure demonstrates the excellent correspondence between $4 \mathrm{D}$ and $5 \mathrm{D}$ approaches to calculating the cross sections. The four KK EVA MCHM cross section perfectly matches the full ECA MCHM cross section until the energy range approaches the mass of the fourth KK mode, where it begins to deviate slightly. After the fourth KK mode there is a sharp departure from the ECA curve - which encodes the full MCHM - exactly as one would expect. However, the six KK EVA cross section continues to match the ECA curve as we have not yet reached the limit of the KK modes that it includes.

This behaviour is supported by Fig. 4.3, where the cross section is plotted with an increasing number of $\mathrm{KK}$ modes included. As each curve reaches the final KK mode included it deviates sharply from the 5D curve, but flattens out at a smaller value. Smaller deviations from the 5D curve can also be seen when each EVA curve 


\section{Comparison of the MCHM and SM Higgsstrahlung Cross-sections}

Approximation No particle mixing, no widths

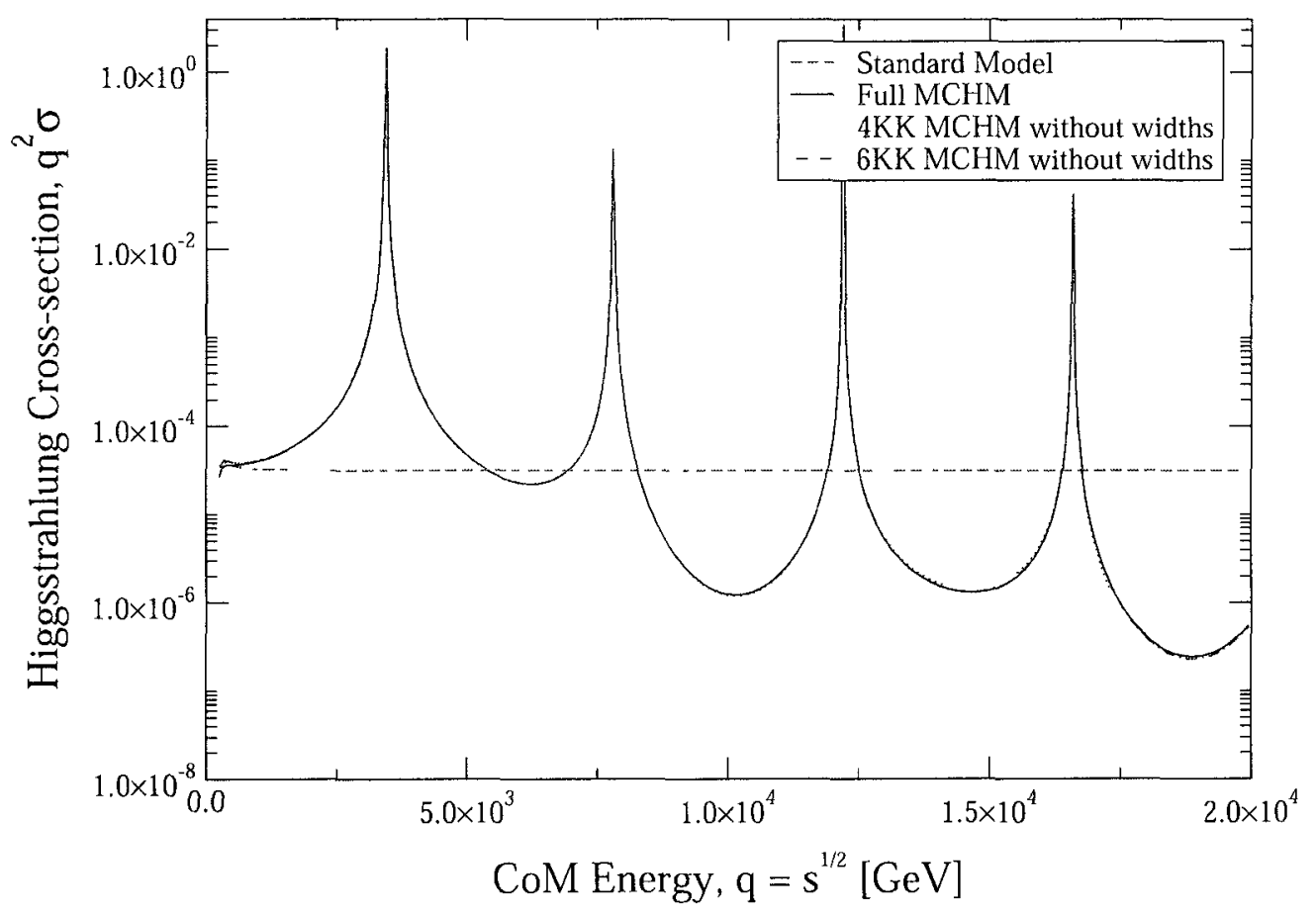

Figure 4.2: The $e^{+} e^{-} \rightarrow Z^{(0)} H$ from 4D (dotted line) and 5D (solid line) plotted with the SM cross section (dashed line). The units of $\sigma$ and $q$ are $[\mathrm{GeV}]^{-2}$ and $[\mathrm{GeV}]$ respectively. Note that the $4 \mathrm{KK}$ and $6 \mathrm{KK}$ curves are virtually indistinguishable from the full MCHM curve until the energy begins to approach their respective cut-off KK masses.

reaches the KK mode preceding the final mode included, indicating that the cross section becomes less accurate as it approaches the cut-off KK mode, as one would expect. This excellent correspondence between the truncated KK cross sections and the full cross section below the cut-off level indicates that the cross section is not substantially altered by KK modes that are much heavier than the center-of-mass $(\mathrm{CoM})$ energy. 


\section{MCHM Cross-section with an increasing number of KK modes}

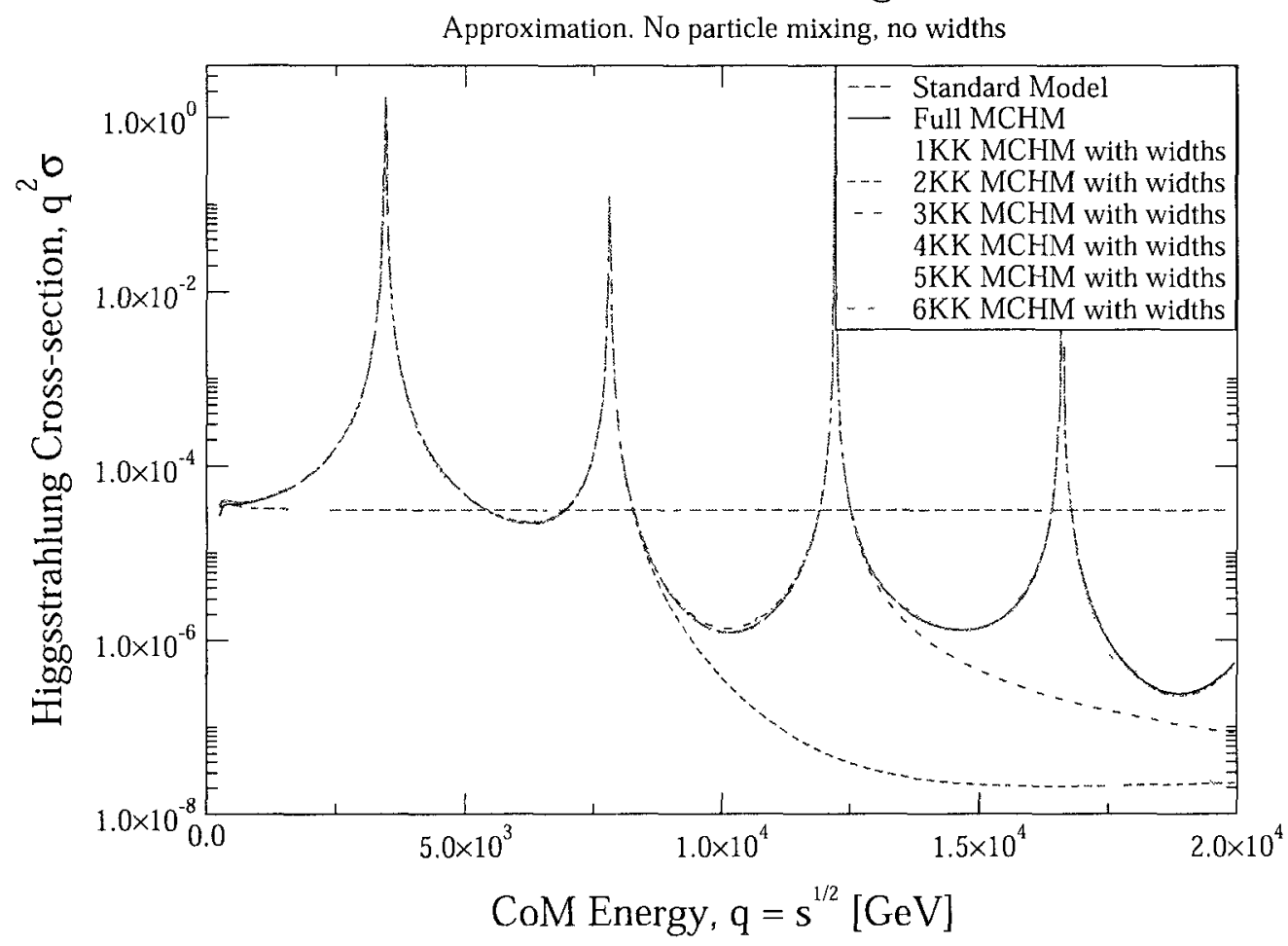

Figure 4.3: A plot of the 4D $e^{+} e^{-} \rightarrow Z^{(0)} H$ cross section times $q^{2}$ including an increasing number of $\mathrm{KK}$ modes, up to 6 (dotted lines). Also plotted is the cross section obtained from 5D (solid line). The units of $\sigma$ and $q$ are $[\mathrm{GeV}]^{-2}$ and $[\mathrm{GeV}]$ respectively.

However, one might note a small discrepancy between the EVA and ECA cross sections at low energy in these two figures; the ECA cross section is substantially smaller than the others through the original SM peak from $200-1000 \mathrm{GeV}$. This feature is due to the fact that the ECA cross section does not include the zero-mode mass of the $Z$ boson. If one plots the EVA cross sections with $M_{Z}^{(0)}=0$ this discrepancy is resolved. Furthermore, all of the MCHM cross sections are slightly higher than that 
of the SM; this is entirely due to the difference in vev value, $v$.

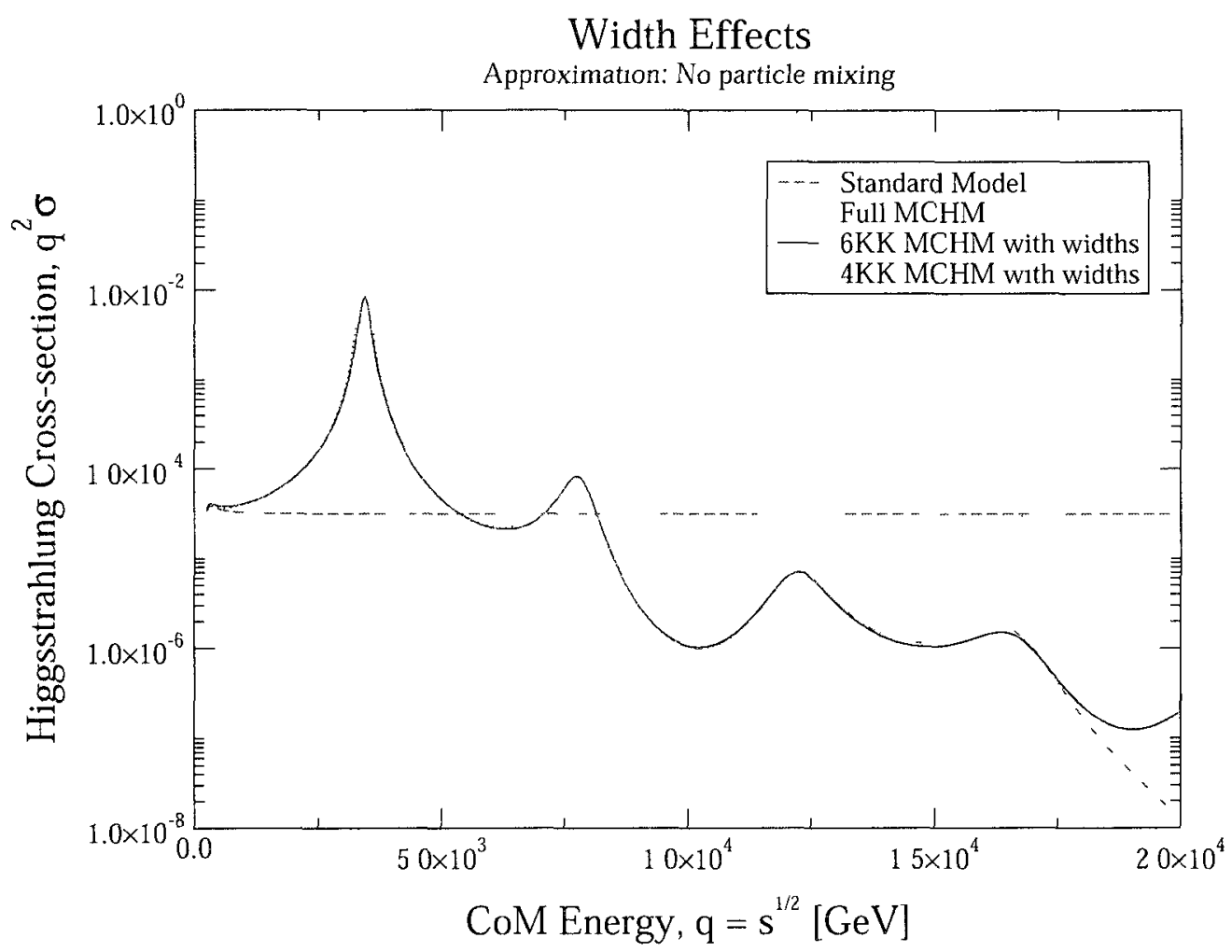

Figure 4.4: The influence of widths on the Higgsstrahlung cross section. The full MCHM does not include widths. Note that these widths do not include KK fermion states.

Fig. 4.4 plots the cross section with widths included in the KK mode propagators. Formulae for the relevant widths can be found in Appendix D. Gauge boson widths are obtained by calculating the boson's decay rate to lighter particles; their addition changes the cross section's divergence at the boson mass into a Breit-Wigner:

$$
\frac{q^{4}}{\left(q^{2}-M_{G}^{(n) 2}\right)^{2}} \rightarrow \frac{q^{4}}{\left(q^{2}-M_{G}^{(n) 2}\right)^{2}+M_{G}^{(n) 2} \Gamma_{G}^{(n) 2}},
$$


We have considered only two body decay states, these include decays to two gauge bosons, a gauge boson and a Higgs, or fermıon antı-fermion parrs Any combination of zero modes and KK modes may contribute, as long as their combined masses are light enough to be kinematically allowed

One can see that the addition of the widths into the cross section does not substantially affect the curve near the first KK resonance, but merely shortens the resonance peak However, as we progress to higher orders of KK modes the widths begin to affect the cross section between resonances, ultimately leading to an increased suppression at higher energies Note that these widths do not include KK fermion decay modes, such modes will further widen the resonances and suppress the cross section, as we will see in section 44 Though the inclusion of widths significantly alters the cross section, it does not change the behaviour of the EVA KK cross section as it approaches the truncation point, we can see that the four KK EVA cross section becomes slightly offset after the thind resonance, and deviates sharply from the six KK EVA cross section after the fourth just as it did before

The most salient feature of Fig 42 , however, is that as the center-of-mass (CoM) energy increases the MCHM cross section in between the KK mode resonances becomes very suppressed in comparison with that of the SM, as expected This is due to the influence of the MCHM s 5D structure From the EVA perspective, it arises due to cancellations between KK contributions to the cross section

To demonstrate this cancellation, let us consider each boson KK mode cross section to be at a point far from the mass resonance, 1 e, in the limit that $M^{2} \ll s^{2}$

\footnotetext{
${ }^{2}$ In truth there is no such point, as there are an infinite number of KK modes so one cannot even theoretically reach an energy highel than all of them However, the behaviour of the cross section at this hypothetical point will indicate its overall behaviour in the troughs between resonances
} 
At such a point, the individual propagators will become equivalent as $s$ overpowers $M_{z}^{(n) 2}$ in the denominator. Therefore the relative difference between the SM and MCHM cross sections will be due to the difference between the SM coupling and the squared sum of the contributing MCHM couplings.

$$
\frac{\sigma_{L, R}^{\mathrm{MCHM}}}{\sigma_{L, R}^{\mathrm{SM}}} \approx\left(\sum_{\imath=1}^{n} \frac{C_{Z f f_{L, R}}^{(\imath)} C_{Z Z H_{L, R}}^{(\imath)}+C_{X f f_{L, R}}^{(\imath)} C_{X Z H_{L, R}}^{(\imath)}}{C_{Z f f_{L, R}}^{(\mathrm{SM})} C_{Z Z H_{L, R}}^{(\mathrm{SM})}}\right)^{2}, \quad \text { if } s \gg M^{(n) 2} .
$$

Note that in the case of the $Z$ couplings $C_{Z}$ the constant coupling factors will be the same as those of the SM, except that the vevs are different. As such, if we neglect $X$ contributions the ratio of MCHM to SM couplings is proportional to the ratio of the vev and the fifth dimension integrals defined in Eqs. (4.9-4.10),

$$
\frac{\sigma_{L, R}^{\mathrm{MCHM}}}{\sigma_{L, R}^{\mathrm{SM}}} \approx\left(\sum_{\imath=1}^{n} \frac{v}{v_{\mathrm{SM}}} Z_{Z Z H}^{2,0} Z_{Z f f}^{\imath}\right)^{2}, \quad \text { if } s \gg M^{(n) 2} .
$$

For the purpose of examining the overall mathematical structure and behaviour, we will consider sums of up to twenty KK modes. However, it should be noted that at some point above $4 \pi M_{k k}$ our effective theory will begin to break down; the strong interactions of the CFT will come into play and perturbation theory will no longer provide an accurate picture of the physics. As such, the details of results above the tenth KK mode may not be a true representation of the physics involved.

As the number of included $\mathrm{KK}$ modes in this sum increases, the MCHM squared sum decreases sharply in comparison to the SM coupling, as seen in Figures 4.5 and 4.6 for the left- and right-handed sums respectively. The first reason for this behaviour is related the the form of the integrals $Z_{Z Z H}^{i, 0} Z_{Z f f}^{\imath}$. Due to the Bessel functions in the gauge boson profiles, these integrals are oscillatory functions of the mass $m$ centered about zero. When evaluated at the gauge masses, the odd order KK modes turn out to have negative couplings, while the even order KK modes have even couplings. 


\section{Behaviour of the squared Higgsstrahlung coupling sum}

Approximation: No particle mixing (left-handed)

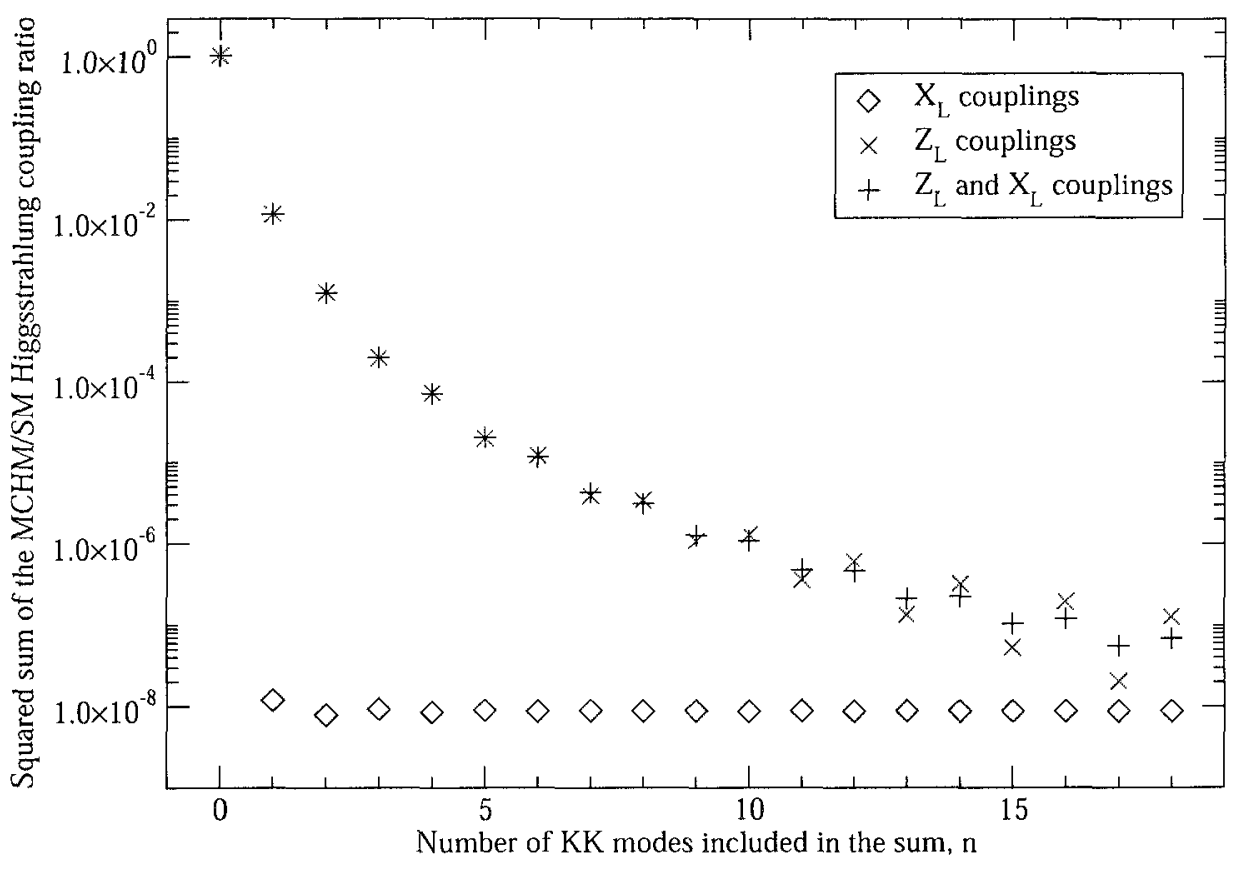

Figure 4.5: The ratio of the squared sum of the left-handed Higgsstrahlung interaction couplings with respect to the left-handed SM Higgsstrahlung coupling plotted versus the number of included KK modes.

The second reason for the coupling sum behaviour is that the first $Z \mathrm{KK}$ mode happens to have an overall coupling roughly ten percent larger than the zero mode in magnitude, but opposite in sign. As such, adding in the first KK mode's contribution completely cancels the zero mode; the coupling sum becomes negative, with about a tenth of the magnitude of the zero mode coupling. Adding the second KK mode -which has a positive coupling - cancels the remainder of the first two, and yields a positive sum of even smaller magnitude. This procedure continues, with the coupling sum oscillating about zero but becoming smaller in magnitude as more and more $\mathrm{KK}$ 
modes are added in When the sum is squared, the negative sum values are flpped up into the positive quadrant, where they fall into line with the positive sum values This creates the relatively smooth downward curve seen in the figures

However, one will note that on the right side of the figures the points begin to fall out of line and spht into two tails Mathematically this occurs because the alternating sequence is not perfectly centered about zero, the negative sum values become smaller in magnitude more quickly than the positive values, indicating that the sum sequence has a non-zero limit As such, in Figs 45 and 46 the curve of squared summation values divides into two lines, one representing the squares of negative values, while the other corresponds to positıve values Eventually a point will occur where new negatıve additions become too small to cancel the previous sum, this is the low point seen in 46 From this point on the sum will remain positive, and will approach a non-zero value As long as the $c$ parameter is relatively large as it must be for the light electron - this summation limit will be much smaller than 1 Incoming additions to the sum will still add and subtract from it, causing it to oscillate about the summation limit as it approaches it

It is at this point that the contributions of the $X$ couplings become important ${ }^{3}$ We can see in Fig 45 that the combined left-handed sum of $Z$ and $X$ couplings does not spht as rapidly as the sum of only the $Z$ contributions This is due to the fact that the overall left-handed coupling coefficients ${ }^{4}$ of the Higgsstrahlung interaction with an $X$ boson propagating are of equal magnitude but opposite sign to those when

\footnotetext{
${ }^{3}$ Of theoretical importance that is This behaviour occurs at energies too high to be relevant to our cross-section plots or further analysis

${ }^{4} \mathrm{By}$ coupling coefficients we mean the constant coefficients of the coupling that are not related to the integral over the fifth dimension
} 
$Z$ propagates Because $T^{3_{L}}=T^{3_{R}}$ for a left-handed electron, ${ }^{5}$ and $g_{x}^{2}=g_{z}^{2} \cos 2 \theta_{w}$, the $X$ coupling coefficients are

$$
g_{x}^{2} \frac{\left(Q-T^{3_{L}}\right) \sin ^{2} \theta_{w}-T^{3_{R}} \cos ^{2} \theta_{w}}{\cos 2 \theta_{w}}=g_{z}^{2}\left(Q \sin ^{2} \theta_{w}-T^{3_{L}}\right),
$$

which is the negative of the $Z$ coupling coefficients

The only difference between the two couplings is the value of the integrals over the fifth-dimensional components, $Z_{Z Z H}^{20} Z_{Z f f}^{2}$ and $Z_{X Z H}^{20} Z_{X f f}^{2}$ As it happens, the sums of these integrals approach similar values as more KK modes are included, as demonstrated in Fig 45, allowing the $X$ contribution to cancel that of the $Z$ boson The sum of both the $X$ and $Z$ left-handed contributions to the Higgsstrahlung interaction will have a limit much smaller than that of the sum of the $Z$ contributions alone The cancellation is not perfect, and the sum will still approach a smaller nonzero value eventually, but the effects of the non-zero limit - 1 e the splitting - will not become apparent untıl much higher energies are reached and many more KK modes are included

However, mcluding the $X$ couplings in right-handed sums does not cause a reduction in the sum limit, in fact, one will note from Fig 45 that their addition causes the deviation to increase This is due to the fact that for right-handed electrons $T^{3_{L}}=0$ and $T^{3_{R}}=-1=Q$, so that the right-handed $X$ coupling coefficients are

$$
g_{x}^{2} \frac{\left(Q-T^{3_{L}}\right) \sin ^{2} \theta_{w}-T^{3_{R}} \cos ^{2} \theta_{w}}{\cos 2 \theta_{w}}=g_{x}^{2} Q=g_{z}^{2} Q \cos 2 \theta_{w}
$$

When added to the $Z$ coupling of $-g_{z}^{2} Q\left(\sin ^{2} \theta_{w}\right)$, the total becomes $-g_{z}^{2} Q\left(\cos ^{2} \theta_{w}\right)$ Not only is this non-zero, but $\cos ^{2} \theta_{w}>\sin ^{2} \theta_{w}$, which is why the deviation in the

\footnotetext{
${ }^{5}$ If the Higgsstrahlung process wele to occur with up-type fermions incoming, rather than downtype, the right-handed sum would approach zero but the left-handed would not
} 


\section{Behaviour of the squared Higgsstrahlung coupling sum}

Approximation: No particle mixing (right-handed)

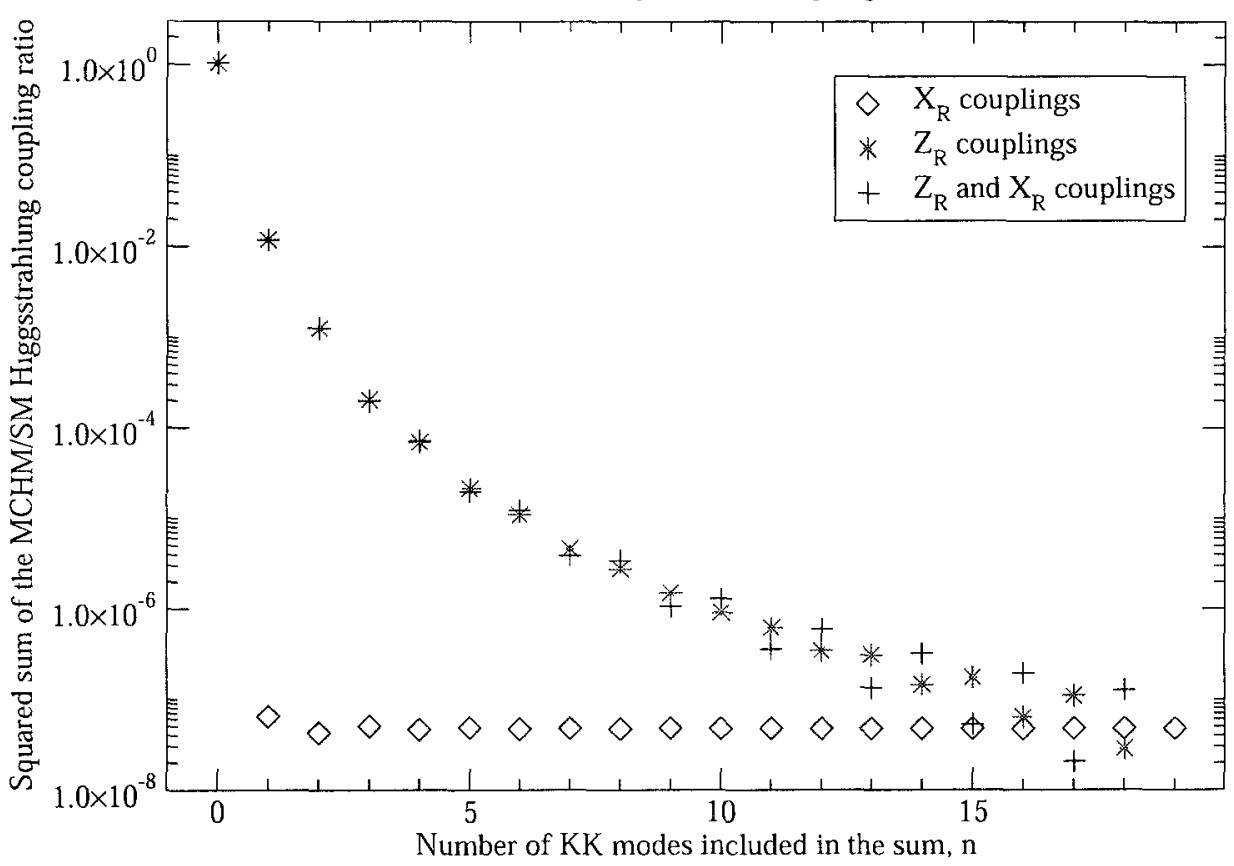

Figure 4.6: The ratio of the squared sum of the right-handed Higgsstrahlung interaction couplings with respect to the right-handed SM Higgsstrahlung coupling plotted versus the number of included KK modes.

tails increases; the combined $X, Z$ sum approaches a larger limit value than the sum with only $Z$ contributions.

It is interesting to note that the left-handed sum is mostly independent of the value of $c$. When $c$ is small $(<0.5)$, both the $X$ and $Z$ contributions sum to large values, as the fermion profiles are located farther away from the Planck brane and couple more strongly with the bosons. In this case, as discussed above, the $X$ sum cancels that of the $Z$ such that the resulting sum is small. When $c$ is large, the $X$ contributions are largely negligible as the fermions are very close to the Planck brane 
where the $X$ profile is zero However, the $Z$ contributions alone still sum to a limit on the same order of magnitude as that when $c$ was larger Furthermore, it seems that although the sum limit is small for all $c$, it is also non-zero for all $c$, if one explicitely constrains the fermions to the Planck brane, the alternating sequence of the sum still demonstrates the slight offset from the ongin, suggesting that the ultmate limit is still non-zero In fact, visually there is no difference between Fig (4 5) and an analogous plot with the fermions constrained to the Planck brane

The primary influence of $c$ is on the right-handed sums, which may be quite large (greater than 1$)$ for small values $(c<0,5)$, where both the $X$ and $Z$ sums are large and do not cancel As the ratios of the $Z$ MCHM couplings to SM couplings are the same for both right and left chirahties, as $c$ become large - and the $X$ contributions become negligible - the right-handed sum becomes equivalent to the left, as both obey $\mathrm{Eq}(421)$

As seen in Fig 4 7, the suppression that results from this KK coupling cancellation is initially stronger than that of an exponential, but becomes less so as the mass of the largest included KK mode increases The suppression with respect to mass can be parametrized by a function of form $y=x^{a} \exp \left[b+c(\ln x)^{2}\right]$, this fit was obtained by taking the logarithm of both varıables, fitting a second order polynomial, and then reversing the transformation The form of the fit suggests an initial exponential influence to the suppression of the cross section, however, the exponential factor is mitigated by both the power factor $x^{a}$ and the logarithm in its argument as the energy increases Indeed, the fit function can equivalently be expressed without an exponential as $y=d x^{a+c \ln z}$, where the constant $d=\exp b$

Fig 47 depicts the cross-section behaviour more accurately than a plot versus the number of KK modes, as the cross section spaces KK modes based on their mass, 


\section{Behaviour of the squared Higgsstrahlung coupling sum}

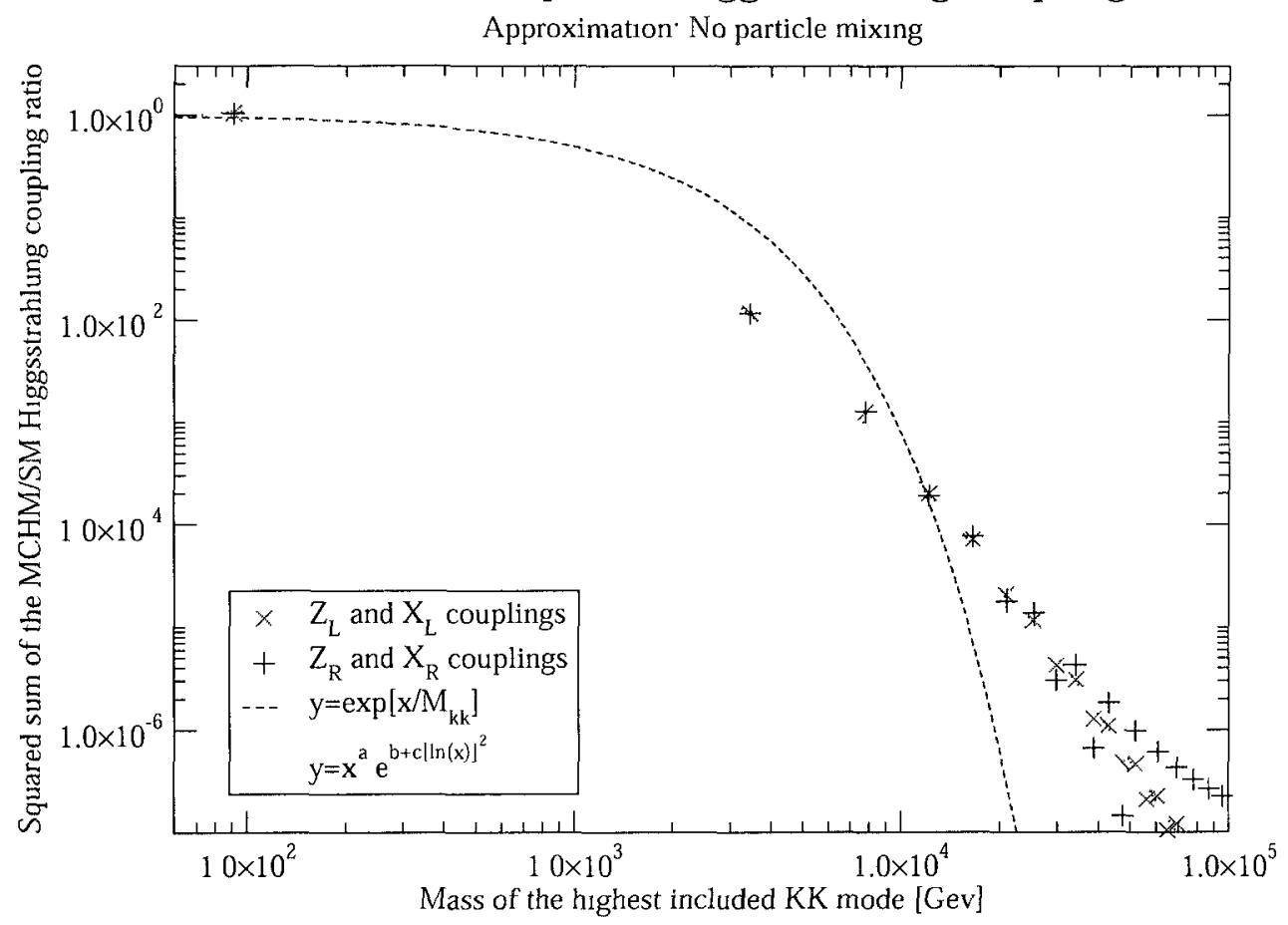

Figure 4.7: The squared sum of Higgsstrahlung to SM coupling ratio (both left- and righthanded) plotted on a log-log scale versus the mass of the largest included KK mode. The paramcters of the fit arc $a=3.7 \pm 0.2, b=-8.8 \pm 0.9$ and $c=-0.40 \pm 0.01$.

not their order. However, it is interesting to note that when plotted on a log-log scale versus $x=n+1$ the squared sums are linear before the splitting behaviour begins, as seen in Fig. (4.8); this indicates a power-law relationship, $y=c x^{b}$, where $b<0$ as the slope is negative. Therefore the relative suppression of the $n$ KK mode squared sum with respect to the $n-1 \mathrm{KK}$ mode squared sum is described by the following 


\section{Behaviour of the squared Higgsstrahlung coupling sum}

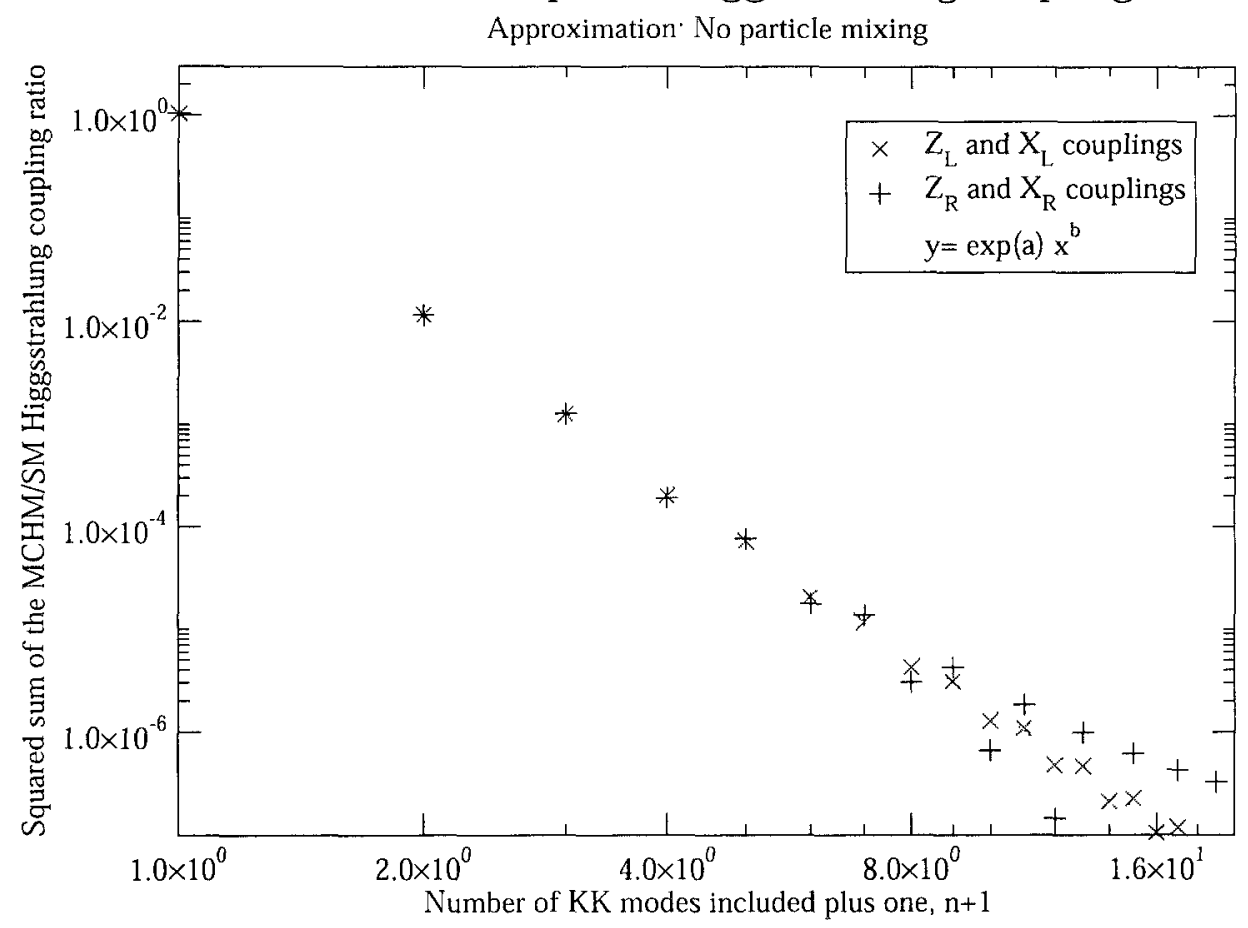

Figure 4.8: The squared sum of Higgsstrahlung to SM coupling ratio plotted on a log-log scale versus the number of $K K$ modes $n$ plus one. The parameters of the fit are $a=-0.6 \pm 0.2$ and $b=-5.50 \pm 0.07$.

relationship

$$
\frac{\left(\sum_{i=0}^{n} C_{\imath}\right)^{2}}{\left(\sum_{l=0}^{n-1} C_{\imath}\right)^{2}} \stackrel{(=\jmath-1}{\longrightarrow} \frac{\left(\sum_{\jmath=1}^{n+1} C_{\jmath}\right)^{2}}{\left(\sum_{\jmath=1}^{n} C_{\jmath}\right)^{2}}=\frac{a(n+1)^{-b}}{a n^{-b}}=\left(\frac{n}{n+1}\right)^{b}
$$

\subsubsection{Coupling extraction}

As with any model, it is important to determine how well the characteristic behaviour of the couplings can be identified at a collider. Let us consider the characterizability 
of the MCHM of $e^{+} e^{-} \rightarrow Z^{(0)} H$ at the proposed International Linear Collider (ILC) and Compact LInear Collıder (CLIC) Both of these accelerators will collide electrons with positrons

The ILC is the more developed of the two, it may be possible to commence construction as early as 2012 It is hoped that ILC will provide complementary data to that of the LHC The ILC proposes to scan center-of-mass (CoM) energies from 200 to $500 \mathrm{GeV}$ - with the possibility of upgrading to $1 \mathrm{TeV}$ and is expected to attain a luminosity of $500 \mathrm{fb}^{-1}$ over 4 years $[58,59]$ CLIC, on the other hand, will require a great deal more research and development before construction is possible, however, it is expected to reach much higher energies - scanning from 05 to $5 \mathrm{TeV}$ with an optimum energy of $3 \mathrm{TeV}$ with twice the luminosity of the ILC [60]

In Table 41 we have tabulated the relevant statistıcs for the MCHM Higgsstrahlung cross section without mixing or widths - measured at $500 \mathrm{GeV}, 1 \mathrm{TeV}$, $3 \mathrm{TeV}$ and $5 \mathrm{TeV}$ with a lummosity of $500 \mathrm{fb}^{-1}$ The expected number of events is found by multiplying the cross section by the luminosity

Note that these statistıcs include all possible decay modes of the $Z^{(0)} H$ final state Roughly twenty percent of the $Z$ decays will be invisible, as they involve neutrinos which cannot be detected However, using Higgs decay data it is possible to reconstruct whether or not a given final state resulted from a $Z^{(0)}$ decay, and thus the Higgsstrahlung process This reconstruction would allows us to compensate for some of the lost percentage It could also be compensated for by increasing the luminosity by 20 percent, or a combination of both strategies

As a first scenarıo, let us assume that the masses and widths of both $Z^{(0) 6}$

\footnotetext{
${ }^{6}$ The ero mode $Z$ mass and widths have in fact been precisely measured at LEP
} 
Table 41 Cross-section data used for coupling extraction

\begin{tabular}{|c|c|c|c|c|}
\hline $\begin{array}{c}\text { COM } \\
\sqrt{s}[\mathrm{TeV}]\end{array}$ & $\begin{array}{c}e^{+} e^{-} \rightarrow Z^{(0)} H \\
\text { cross section, } \\
\sigma \mathrm{fb}]\end{array}$ & $\begin{array}{c}\text { Number of } \\
\text { Events, } N\end{array}$ & $\begin{array}{c}\text { Statistical } \\
\text { Eror on } N, \\
\sqrt{N}\end{array}$ & $\begin{array}{c}\text { Statistical } \\
\text { Error on } \sigma, \\
\sigma[\mathrm{fb}]\end{array}$ \\
\hline \hline 05 & 6018 & 30089 & 173 & $346910^{-1}$ \\
\hline 1 & 1581 & 7903 & 89 & $177810^{-1}$ \\
\hline 3 & 2609 & 13046 & 114 & $228510^{-1}$ \\
\hline 5 & $712810^{-1}$ & 356 & 19 & $377610^{-2}$ \\
\hline
\end{tabular}

the $\mathrm{LHC}^{6}$ We would like to know whethes the first MCHM $Z$ KK mode could be identified at the ILC or CLIC through the Higgsstrahlung interaction, and what 1dentifying information about the coupling could be determined

Fig 49 plots a two-parameter $\Delta \chi^{2}$ test variable

$$
\Delta \chi^{2}=\sum_{\imath} \frac{\left(\sigma_{\mathrm{th}}^{2}-\sigma^{2}\right)^{2}}{\Delta \sigma^{2}}
$$

where $\sigma$ and $\Delta \sigma$ are listed in the table above, $\imath=1,2 n$ denotes the $n$ differcnt energy values at which $\sigma$ was measured and

$\sigma_{\mathrm{th}}\left(s, C_{z}^{(0)}, C_{z}^{(1)}\right)=\operatorname{Coef}[s]\left|\frac{C_{z}^{(0)}}{s-M_{z}^{(0) 2}+\imath \Gamma_{Z}^{(0)} M_{Z}^{(0)}}+\frac{C_{z}^{(1)}}{s-M_{z}^{(1) 2}+\imath \Gamma_{Z}^{(1)} M_{Z}^{(1)}}\right|^{2}$

is a test function for the unpolarized $e^{+} e^{-} \rightarrow Z^{(0)} H$ cross section Here Coef $[s]$ denotes the usual SM coefficients of the $e^{+} e^{-} \rightarrow Z^{(0)} H$ cross section and $C_{z}^{(0)}, C_{z}^{(1)}$

\footnotetext{
${ }^{6}$ The zero mode $Z$ mass and widths have in fact been precisely measured at LEP
} 


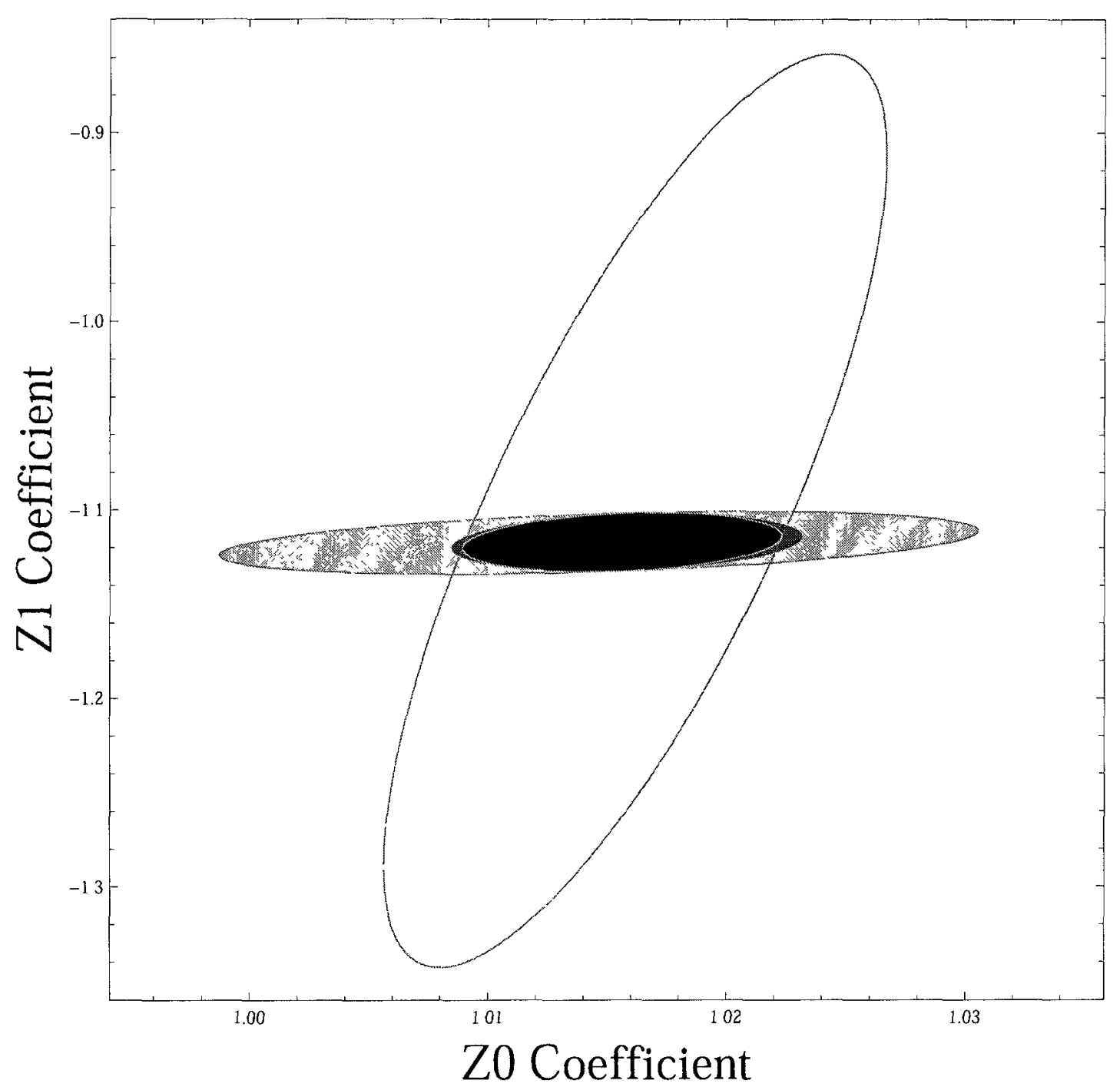

Figure 4.9: Extraction of $Z^{(0)}$ and $Z^{(1)}$ couplings from a $\chi^{2}$ at 95 percent confidence using the following data points: 0.5 and $1 \mathrm{TeV}$ (light grey, vertical); 1 and $3 \mathrm{TeV}$ (medium grey, horizontal); 0.5 and $3 \mathrm{TeV}$ (dark grey, centered); 0.5, 1, and $3 \mathrm{TeV}$ (black, centered). The coupling ratios are expected to be $C_{Z}^{(0)}=1.016, C_{Z}^{(1)}=-1.124$. 
denote the magnitude of the $Z^{(0)}, Z^{(1)}$ couplings relative to the SM coupling By 'couplings' here we mean the product of both the $Z^{(n)} \vec{f} f$ and the $Z^{(n)} Z^{(0)} H$ couplings, or the overall interaction coupling

We can see from Fig (49) that even at the ILC, which operates at energies much lower than the $Z^{(1)}$, the first $Z \mathrm{KK}$ mode coupling can clearly be differentiated from zero, and therefore identified through the Higgsstrahlung process Furthermore, the two-point $\chi^{2}=599$ parameter space resulting from 05 and $1 \mathrm{TeV}$ measurements clearly differentiates $C_{Z}^{(0)}$ from 1 , this is due to the difference between the MCHM and SM vevs, and indicates that the MCHM $Z^{(0)}$ could be distinguished from the SM $Z^{(0)}$ at the ILC

The 05 and $1 \mathrm{TeV}$ parameter space relevant to the ILC also indicates that the $Z^{(1)}$ has the opposite sign of the SM coupling, however, it does not constrain its magnitude very precisely CLIC would be able to show that $C_{Z}^{(1)}$ is both negative and roughly ten percent larger than the zelo-mode coupling by providing data at the $3 \mathrm{TeV}$ scale, recall that the mass of $Z^{(1)}$ is $344 \mathrm{TeV}$ for our parameter chorce This is extremely important, as the sign and magnitude of the first $Z \mathrm{KK}$ coupling are key characterıstics of the MCHM which lead to the suppression of the Higgsstrahlung cross section

The best constraints for both parameters would be obtained from a data set that includes points at both the 05 and the $3 \mathrm{TeV}$ range If the former is omitted, $C_{Z}^{(0)}$ cannot be differentrated fiom $C_{\mathrm{SM}}$, while if the latter is omitted the magnitude of $C_{Z}^{(1)}$ becomes vague Attempts to include a $5 \mathrm{TeV}$ data point yleld a null parameter space, as the test function including only one KK mode deviates from the true cross section as energies move past the first KK resonance and begin to approach the second This would provide evidence for the second KK mode 


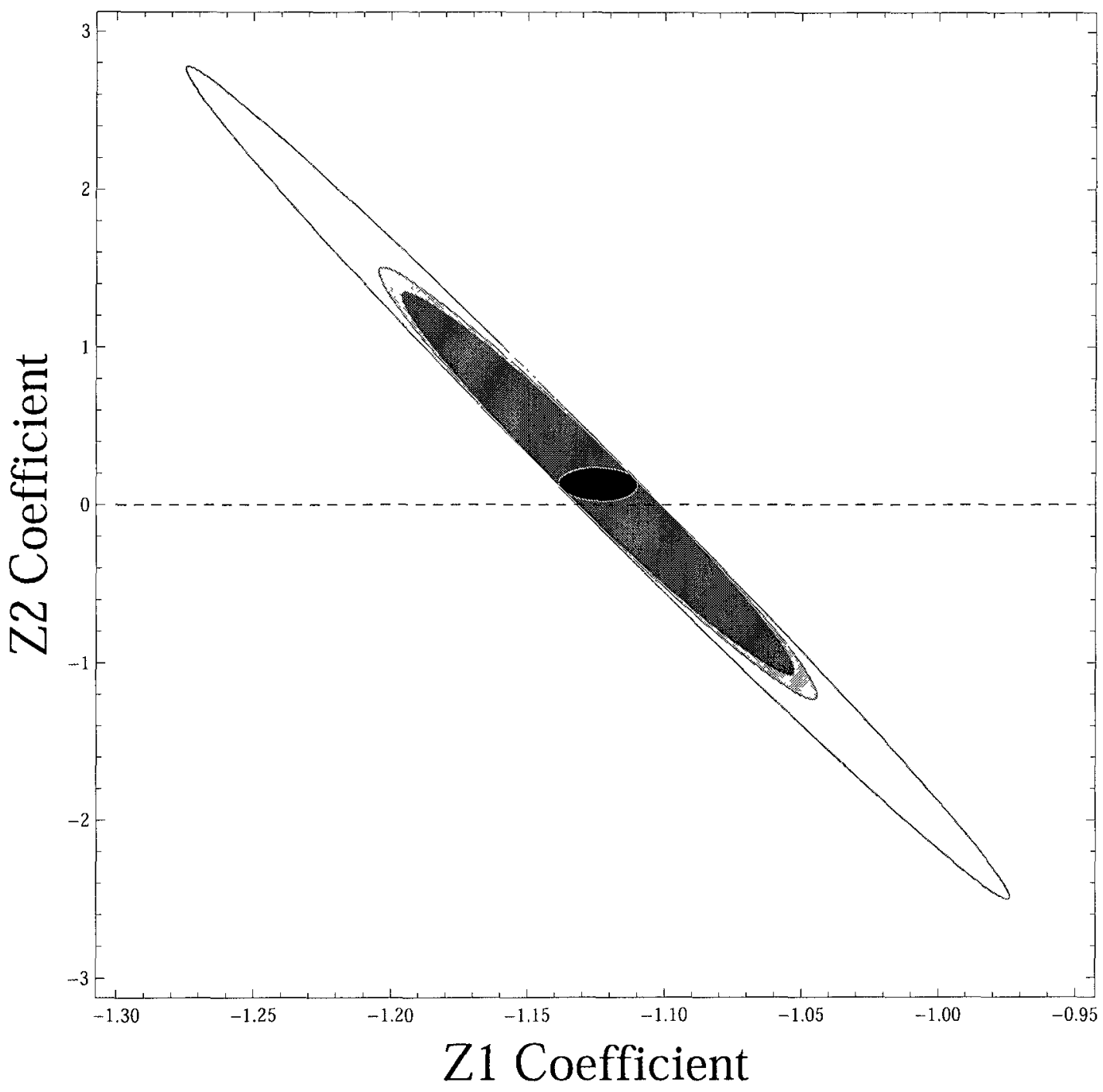

Figure 4.10: Extraction of $Z^{(1)}$ and $Z^{(2)}$ couplings from a $\chi^{2}$ at 95 percent confidence. The data points used, from the outside in, are: 0.5 and $3 \mathrm{TeV} ; 1$ and $3 \mathrm{TeV} ; 0.5,1$, and $3 \mathrm{TeV} ; 0.5,1,3$ and $5 \mathrm{TeV}$. The coupling ratios are expected to be $C_{Z}^{(1)}=-1.124$, $C_{Z}^{(1)}=0.143$. 
As a second scenario, let us assume that the $Z$ zero mode coupling could also be determined at the LHC from Higgs production and decay measurements, along with the mass and width of the second $Z \mathrm{KK}$ mode at $78 \mathrm{TeV}$ Would it be possible to identify the coupling of this KK mode in such a case? Figure 410 plots the same $\chi^{2}$ of $\mathrm{Eq}(425)$ in a parameter space of the $Z^{(1)}$ and $Z^{(2)}$ couplings, $C_{Z}^{(1)}$ and $C_{Z}^{(2)}$, with the new test function

$$
\sigma_{\mathrm{th}}\left(s, C_{Z}^{(1)}, C_{Z}^{(2)}\right)=\operatorname{Coef}[s]\left|\sum_{n=0}^{2} \frac{C_{Z}^{(n)}}{s-M_{z}^{(n) 2}+\imath \Gamma_{Z}^{(n)} M_{Z}^{(n)}}\right|^{2},
$$

where $C_{z}^{(0)}$ is now considered to be a known quantity In this figure the $\chi^{2}$ test is performed at the 95 percent confidenes level, and is calculated from 2 data points (500 GeV and $3 \mathrm{TeV}, 1$ and $3 \mathrm{TeV}$ ), 3 data points (500 GeV, $3 \mathrm{TeV}$, and $5 \mathrm{TeV}$ ) and 4 data points (500 GeV, $1 \mathrm{TeV}, 3 \mathrm{TeV}, 5 \mathrm{TeV}$ ) Again, as long as a $3 \mathrm{TeV}$ data point is included, the parametcr spaces indicate that the first KK mode has the opposite sign and a slightly larger magnitude than that of the SM coupling We can see that only the 4-point region - which includes the $5 \mathrm{TeV}$ point - is clearly distinguishable from zero Thercfore the second KK mode will not be statistically identifiable unless CLIC is pushed to its maximum CoM energy A full analysis of the interaction with backgrounds will be required to precisely determine CLIC's ability to properly identify the second $Z \mathrm{KK}$ mode However, even if the coupling cannot be differentiated from zero, the $5 \mathrm{TeV}$ point parameter space constrains the second $Z \mathrm{KK}$ coupling to be positive and smaller than the first KK mode coupling by a factor of four or more $\left|C_{Z}^{(2)}\right| \lesssim 025\left|C_{Z}^{(1)}\right|$ 


\subsection{Higgsstrahlung with full particle mixing}

\subsubsection{Comparison and analysis of cross sections}

Having completed the analysis in the original approximation, the next step is to investigate the effects induced by including full particle mixing and fermion KK decay modes. To incorporate the full particle mixing we must proceed by means of the EVA approach, making use of the mixed profiles and coupling definitions given in Section 3.2 .5

In the following, we continue to use the original parameters outlined in Table 3.7. As discussed in that section, we will use the same parameters for all of our light fermions; as such, we will choose $c_{1}=c_{2}=c_{3}=0.7, M_{2}=0.627$ and $M_{1}=1.5$ for all multiplets other than those of the third generation quarks. The choice of $M_{2}$ was made by requiring that the down-type fermion mass condition yield the electron mass, as this is the most important light fermion for our purposes.

The introduction of particle mixing does not substantially change the magnitude of couplings relevant to the Higgsstrahlung interaction. Fig. (4.11) compares the Higgsstrahlung cross section without widths both including and neglecting particle mixing; the cross sections are very similar.

The primary difference between the two is the extra resonances which occur between the primary $Z$ KK peaks. These resonances correspond to our $A^{\hat{3}}$ bosons; ${ }^{7}$ due to their mixing with the $Z$ boson KK modes, they are now able to propagate through the Higgsstrahlung interaction, though it was forbidden before EWSB.

The other difference is that the mixed cross section is slightly shifted down-

\footnotetext{
${ }^{7}$ Please refer to Table 3.8 for the $A^{\hat{3}}$ mass spectrum.
} 


\section{MCHM with mixing vs. without mixing (without widths)}

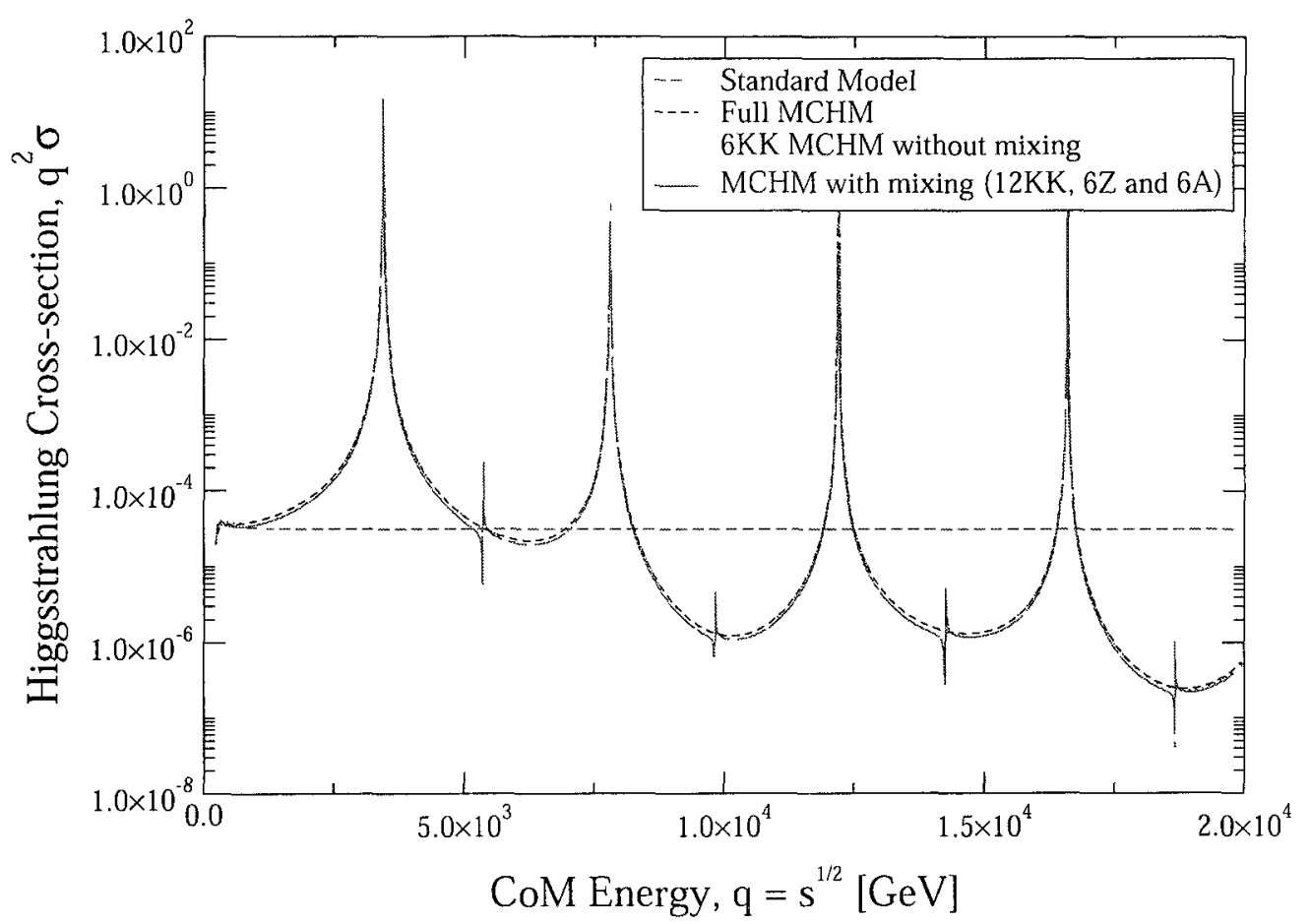

Figure 4.11: The Higgsstrahlung cross section times $q^{2}$ with full mixing but no widths, compared with the approximate cross sections of Fig. (4.2) and the SM.

ward in comparison with the approximate cross section. This effect is entirely due to slight changes in the $Z$ Higgsstrahlung couplings. Both the $Z \bar{f} f$ and $Z Z^{0} H$ couplings individually become slightly smaller after particle mixing - by approximately 3 percent - which leads to an overall suppression of roughly 7 percent in the overall Higgsstrahlung interaction. This slight suppression in the couplings can also be observed in Fig. (4.12), which compares the right- and left-handed squared coupling sums before and after mixing; the centers of the mixed coupling icons (crosses and triangles) are slightly lower than those of the unmixed coupling icons (x's and diamonds). 
In the case of the $X$ couplings the fermion couplings are suppressed by approximately 2 percent after particle mixing, but the $X Z^{0} H$ couplings become stronger by about the same amount. As a result the overall $X$ Higgsstrahlung couplings alternate; odd mixed $X$ KK modes have slightly stronger total couplings than the corresponding unmixed modes, but the even modes are slightly weaker. The overall change is less than one percent for all KK modes. As the change in all of the couplings is small, the $X$ contribution to the cross section is still negligible in comparison to that of the $Z$.

\section{Behaviour of the squared Higgsstrahlung coupling sum}

Comparing mixing with non-mixing results (all boson contributions)

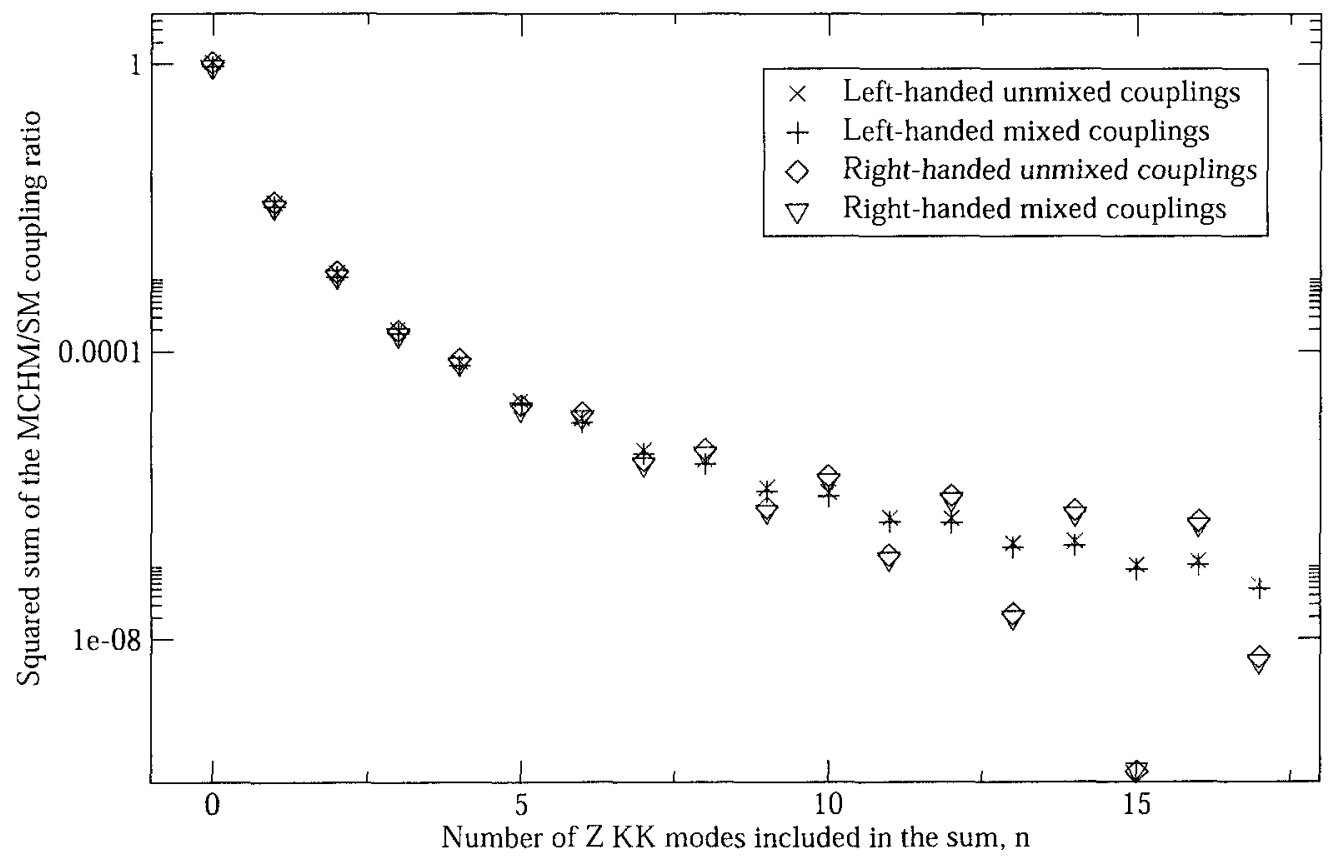

Figure 4.12: A comparison of the square Higgsstrahlung coupling sum $\sim$ divided by the corresponding SM coupling - including particle mixing to the squared sums depicted in figures 4.5 and 4.6 of the previous section. 
As a result, there is virtually no change in the behaviour of the squared coupling sums As depicted in Fig 4 12, there is the same suppression as the number of KK modes increases, and the same deviation in the right-handed couplings indicating that the sum does not ultimately trend to zero The mitial ratıo between the MCHM and SM cross sections displays the same suppression behaviour seen in Sec 434 , the initial suppression displays the same $y=x^{a} \exp \left[b+c(\ln x)^{2}\right]$ behaviour but eventually platcaus at some small value due to the slight offset in the alternatıng sum sequence After this plateau the MCHM cross section would stıll be strongly suppressed compared to the SM, but the suppression would be constant, not exponentıal

By far the primary influence of the particle mixing on the Higgsstrahlung cross section is through the boson decay widths Although the couplings involving SM particles are not greatly affected by the mixing - as the mixing gauge transformation has no effect on the Planck brane - couplings between higher order KK gauge bosons are significantly altered Furthermore, new decay modes become avallable due to couplings between lesser components of the mixed bosons, as previously discussed These new effects greatly increase the decay widths of the propagating bosons, flattening the corresponding resonance peaks in the cross section

In Fig 4 13, a mixed cross section is plotted with the corresponding unmixed 6 KK mode cross section of Fig 44 Both these cross sections include only the boson and SM fermion decay modes, we have not yet included KK fermion decay modes Yet despite the fact that the widths are incomplete, the KK modes beyond $Z^{(1)}$ have become almost completely flat The $A^{3}$ resonances have disappeared entrrely, due to therr small couplings before widths were included Furthermore, the increase in the wldths has further suppressed the cross section 


\section{MCHM with mixing vs. without mixing (with widths)}

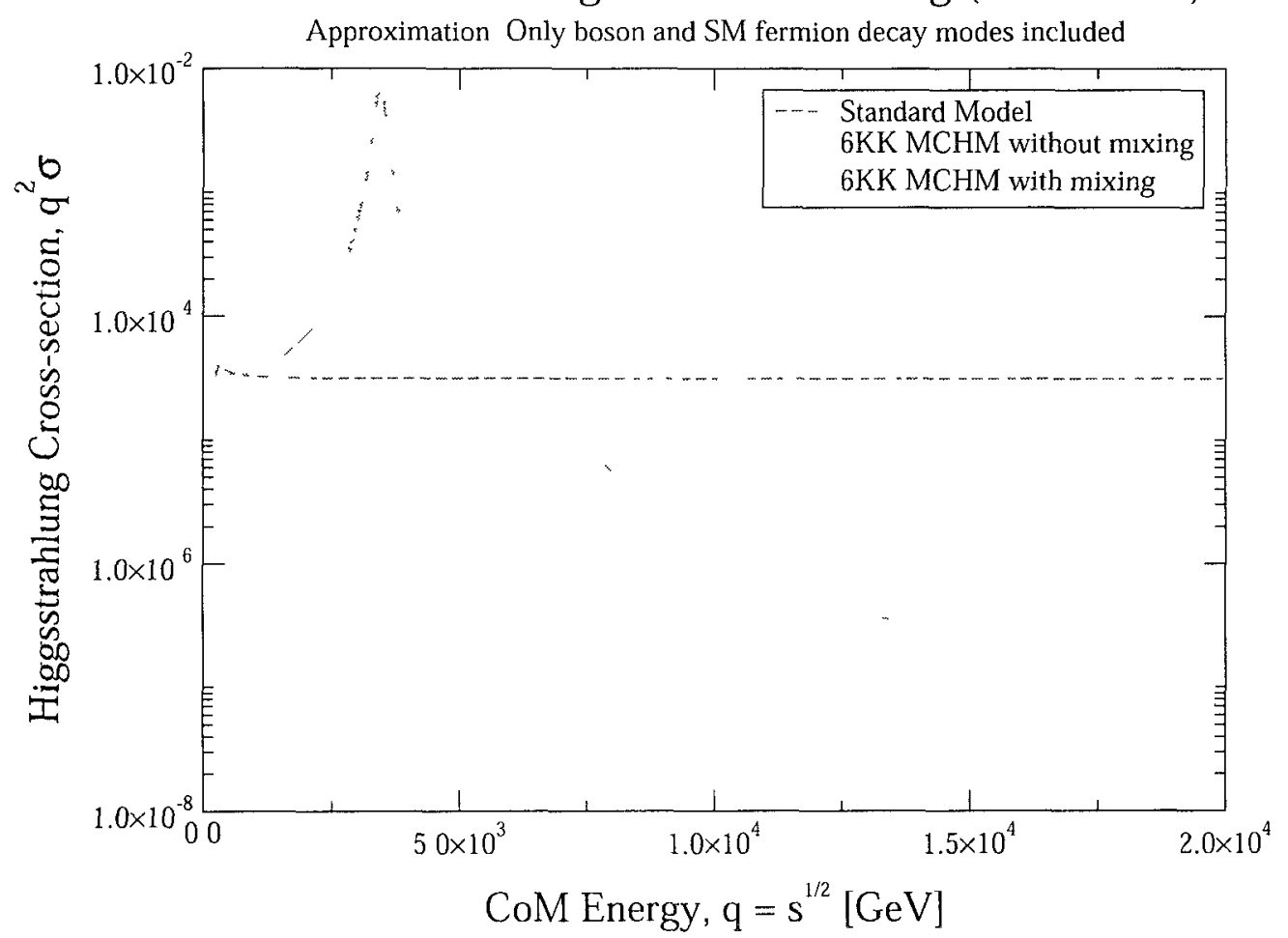

Figure 4.13: The Higgsstrahlung cross section times $q^{2}$ with 6 KK modes, widths and full particle mixing compared with the corresponding cross section without mixing, Fig. 4.4. Only boson and SM fermion decay channels are included in the widths.

However, note that the first KK mode resonance has only been shifted down very slightly, similar to the shift in the overall cross section. As the lightest boson KK mode, the $Z^{(1)}$ cannot decay into other KK bosons; therefore the width of the mixed $Z^{(1)}$ resonance in Fig. 4.13 includes only decays to zero mode particles. Since the couplings of these particles are only slightly shifted by mixing effects, the $Z^{(1)}$ resonance is not greatly altered by the mixing if $\mathrm{KK}$ fermion decay channels are omitted from the width. 
Including the full KK fermion decay modes in the $Z^{(1)}$ width has the result of shortening the peak slightly, as can be seen in Fig 414 The effect is not dramatic, as only the first top quark KK mode is $\operatorname{light}$ enough for the first $Z$ to decay into it 1 e, $M_{t}<05 M_{Z}^{(1)}$ - when $M_{1}=15$ for the light fermions

However, our original choice of this parameter $M_{1}$ was somewhat arbitrary To gain a better idea of the influence of the fermions on the $Z^{(1)}$ width, we also include a full width for the case that $M_{1}=22$, in this case the first neutrino, up and charm KK modes are also light enough to be included The resulting decrease in the resonance peak cross section is small, although numerically the size of the width jumps from $346 \mathrm{GeV}$ to $442 \mathrm{GeV}$ (roughly from ten to thirteen percent of the $Z^{(1)}$ mass) Had we chosen an entırely dıfferent parameter set the first fermıon KK masses could become much smaller, on the order of several hundred $\mathrm{GeV}[4,50]$ Obviously this would significantly alter the boson widths, as such our results are significantly model dependent

Even without lowerıng the first fermın KK masses, the widths of the KK modes beyond the first $Z$ would become even more significantly suppressed if all of the KK fermon decay modes were included Consider the second $Z \mathrm{KK}$ mode, at the very least, its higher mass allows decays to all of the first order fermion KK modes and the second KK modes of the top quark Because of this, it will include decays to many exotic fermions which aren't avarlable to $Z^{(1)}$ As there are 6 sets of fermion multiplets - each containing 20 fermions -- as well as their corresponding chiral partners, one can see that the resulting contribution will be substantial

Even if only the boson decay modes are included, the widths of the $Z \mathrm{KK}$ modes of order $n \geq 2$ are already between thirty and fifty percent of the boson $\mathrm{s}$ mass (for comparison, recall that the the full width of the first $Z \mathrm{KK}$ mode is roughly 


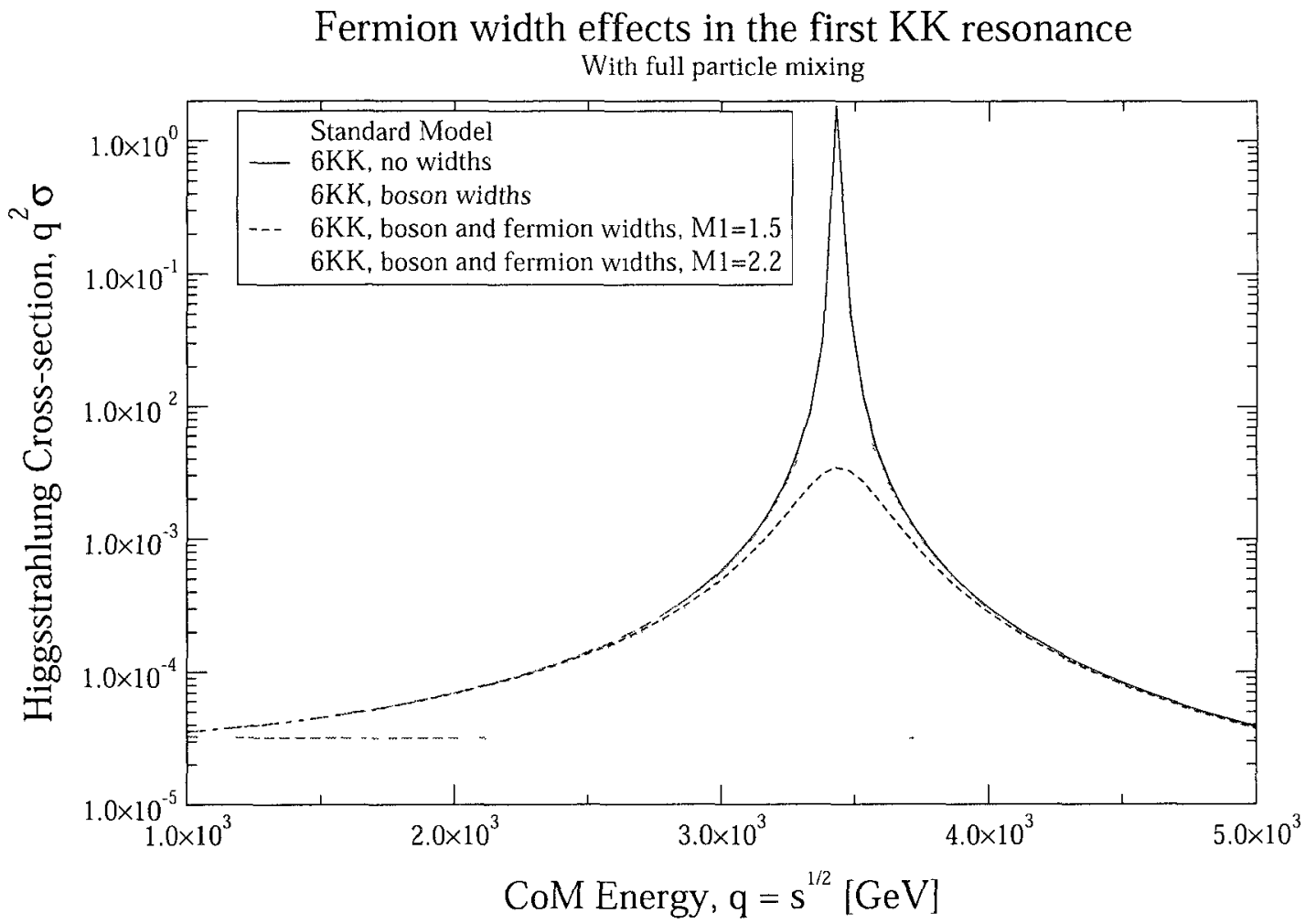

Figure 4.14: The first $Z$ KK resonance peak in the Higgsstrahlung cross section times $q^{2}$. The figure compares cross sections without widths, with only boson widths, and with both boson and fermion widths for $M_{1}=1.5,2.2$.

ten percent of its mass). Once fermions are fully incorporated as well, it seems evident that the widths of higher order KK modes will soon begin to approach or exceed their masses. This indicates that the effective theory is breaking down; we are beginning to reach energies which probe the strongly coupled conformal field theory. At this range, the higher order KK modes become strongly coupled, and perturbation theory can no longer be used. As such, the width calculations are no longer accurate, as they contain terms proportional to couplings involving only heavy - and thus 
predominantly composite - partıcles

However, it should be noted that the Higgsstrahlung cross section itself contains only couplings between KK modes and light SM partıcles The same effect that keeps the masses of these particles light also requires that their couplings to the CFT sector be extremely weak As such, the coupling behaviour of the cross section itself should not suffer from this deficiency It is only the width calculations, which involve couplings between higher order KK modes, which become unreliable

\subsubsection{Coupling extraction}

We will now apply the same coupling extraction proceedure that was performed in Section 415 to the MCHM Higgsstrahlung cross section with full mixing and widths The relevant statistics are tabulated in Table 42 For the record, we are still using the parameter $M_{1}=15$ for the light fermions

Table 42 Cross-section data used for coupling extraction

\begin{tabular}{|c|c|c|c|c|}
\hline $\begin{array}{c}\text { COM } \\
\sqrt{s}[\mathrm{TeV}]\end{array}$ & $\begin{array}{c}e^{+} e^{-} \rightarrow Z^{(0)} H \\
\text { cross section, } \\
\sigma[\mathrm{fb}]\end{array}$ & $\begin{array}{c}\text { Number of } \\
\text { Events, } N\end{array}$ & $\begin{array}{c}\text { Statistical } \\
\text { Error on } N, \\
\sqrt{N}\end{array}$ & $\begin{array}{c}\text { Statistical } \\
\text { Error on } \sigma, \\
\sigma[\mathrm{fb}]\end{array}$ \\
\hline \hline 05 & 5291 & 26455 & 163 & $325310^{-1}$ \\
\hline 1 & 1390 & 6947 & 83 & $166710^{-1}$ \\
\hline 3 & 2151 & 10752 & 104 & $207410^{-1}$ \\
\hline 5 & $581910^{-1}$ & 291 & 17 & $341210^{-2}$ \\
\hline
\end{tabular}




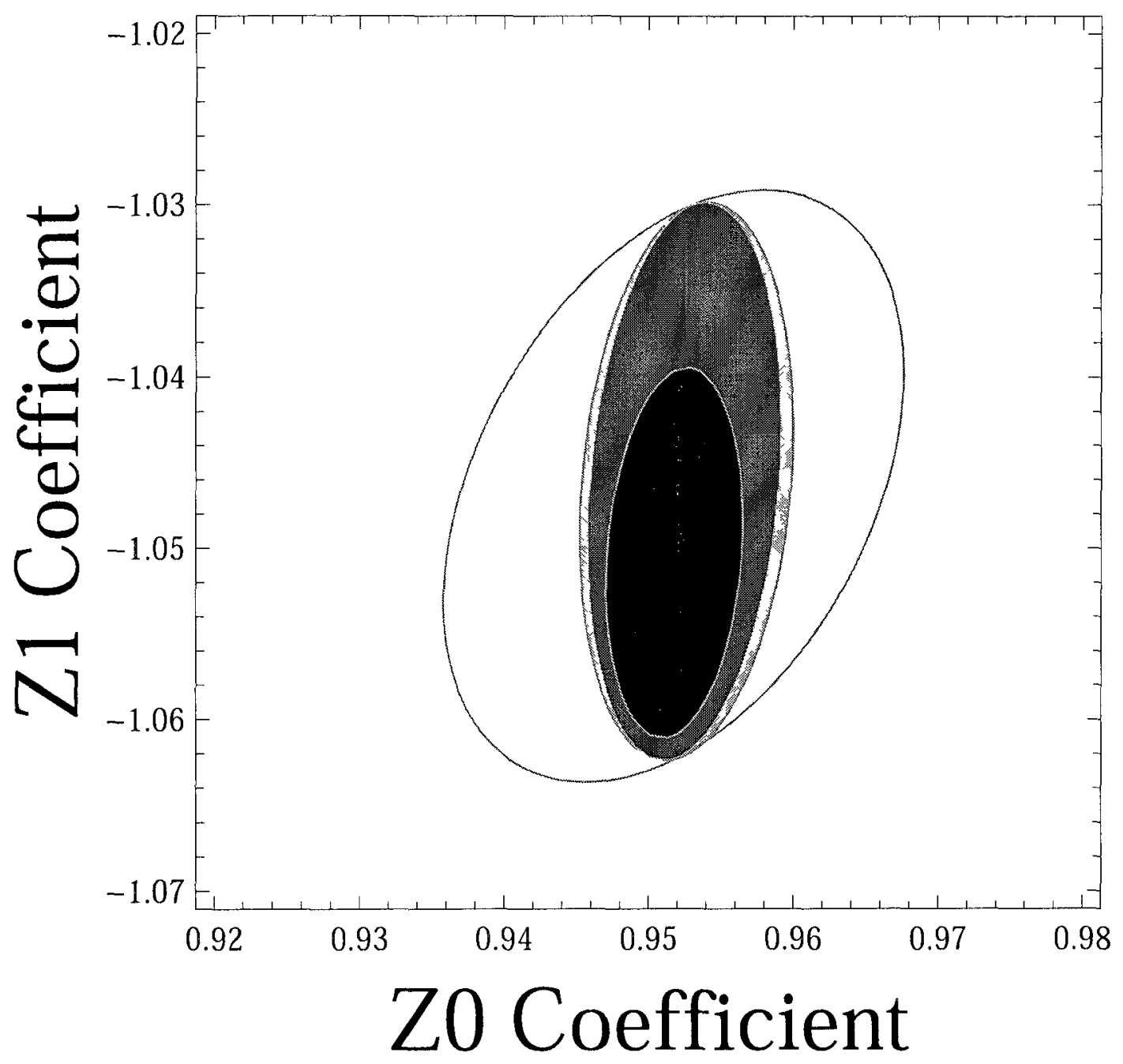

Figure 4.15: Extraction of $Z^{(0)}$ and $Z^{(1)}$ couplings from a $\chi^{2}$ at 95 percent confidence level. The data points used, from the outside in, are: 1 and $3 \mathrm{TeV} ; 0.5$ and $3 \mathrm{TeV} ; 0.5$, 1 , and $3 \mathrm{TeV} ; 0.5,1,3$ and $5 \mathrm{TeV}$. The coupling ratios are expected to be $C_{Z}^{(0)}=0.953$, $C_{Z}^{(1)}=-1.052$, with $M_{1}=1.5$.

Fig. 4.15 is analogous to Fig. 49 , it depicts the different $Z^{(0)}$ and $Z^{(1)}$ coupling paramter spaces produced by a $\chi^{2}$ evaluated at the four energy values. In some 
respects the results are very simlar to those of Fig 4 9, again, the zero-mode coupling can be differentrated from the SM for all data sets, and the first $Z \mathrm{KK}$ coupling is shown to be roughly ten percent larger in magnitude than $C_{Z}^{(0)}$ and of opposite sign However, the new parameter spaces reflect the shift in the Higgsstrahlung couplings due to particle mixing by centering on the new coupling values Both are smaller in magnitude by approximately 7-8 percent respectively, which is consistent with the results found in the previous section Furthermore, both couplings are more strongly constrained

The plot depicted in Fig 416 is almost identical - and extremely similar in appearance - to Fig 410 However, the small central parameter space (black) which includes the $5 \mathrm{TeV}$ point is slightly shifted down in comparison with the plot in the previous section As a result, even at the highest center-of-mass energy of CLIC the second $Z \mathrm{KK}$ mode coupling will not be distinguishable from zero As in the previous section, Fig 416 implies the constiant that the magnitude of $C_{z}^{(2)}$ be approximately one fourth that of $C_{Z}^{(1)}$ However, recall that this cross section does not include the full fermion decay width for $Z^{(2)}$ To determine how changes in the width wll change the constraint, we set the width of the second KK mode equal to its mass, $\Gamma_{Z}^{(2)}=M_{Z}^{(2)}$ (using $M_{1}=22$ ) This plot is depicted in Fig 4 17, one can see that $C_{z}^{(2)}$ is constrained to be smaller than 025 even when the boson's width approaches its mass 


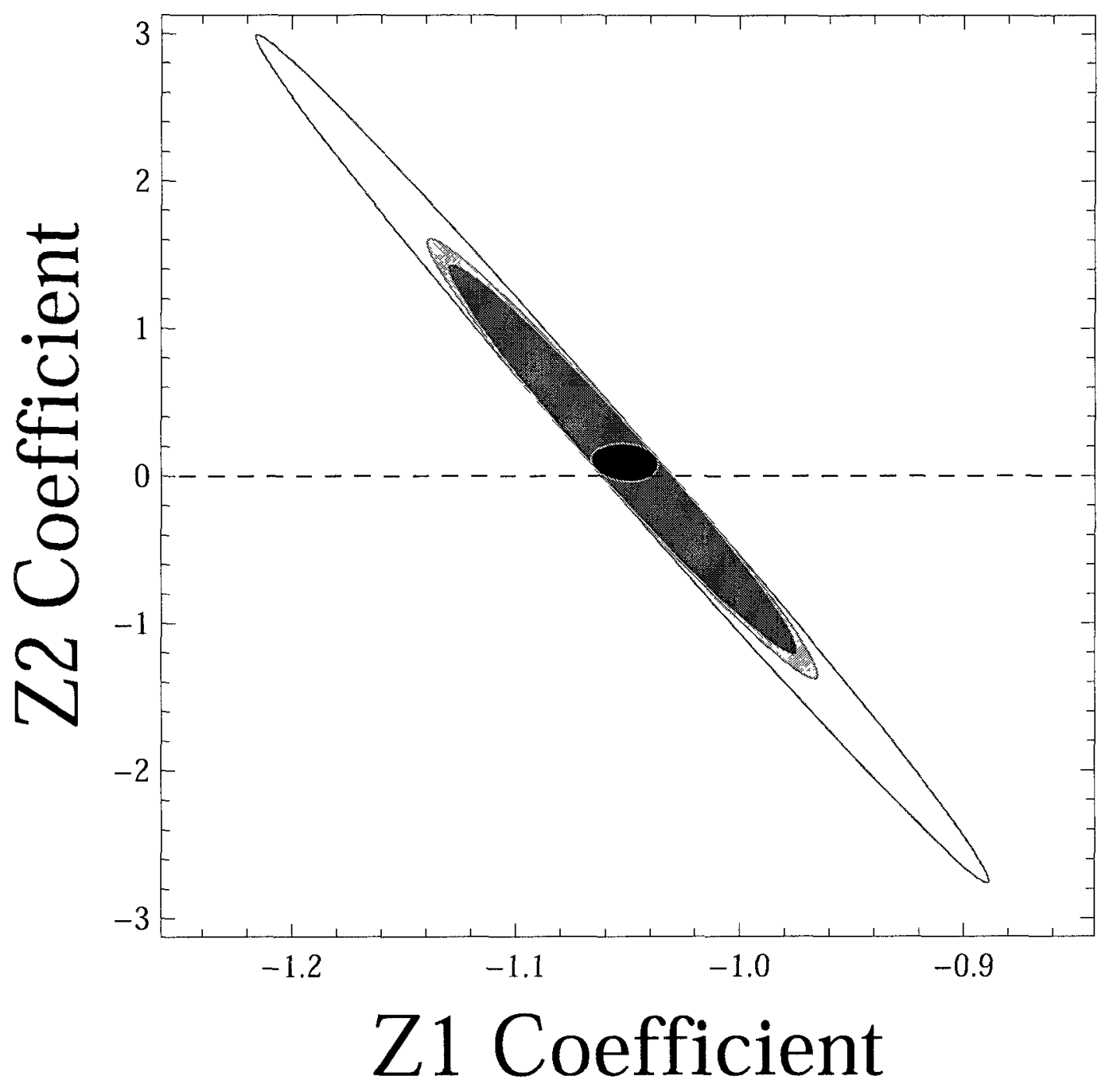

Figure 4.16: Extraction of $Z^{(1)}$ and $Z^{(2)}$ couplings from a $\chi^{2}$ at 95 percent confidence level. The data points used, from the outside in, are: 0.5 and $3 \mathrm{TeV} ; 1$ and $3 \mathrm{TeV}$; $0.5,1$, and $3 \mathrm{TeV} ; 0.5,1,3$ and $5 \mathrm{TeV}$. The coupling ratios are expected to be $C_{Z}^{(1)}=-1.052, C_{Z}^{(2)}=0.134$, with $M_{1}=1.5$. 


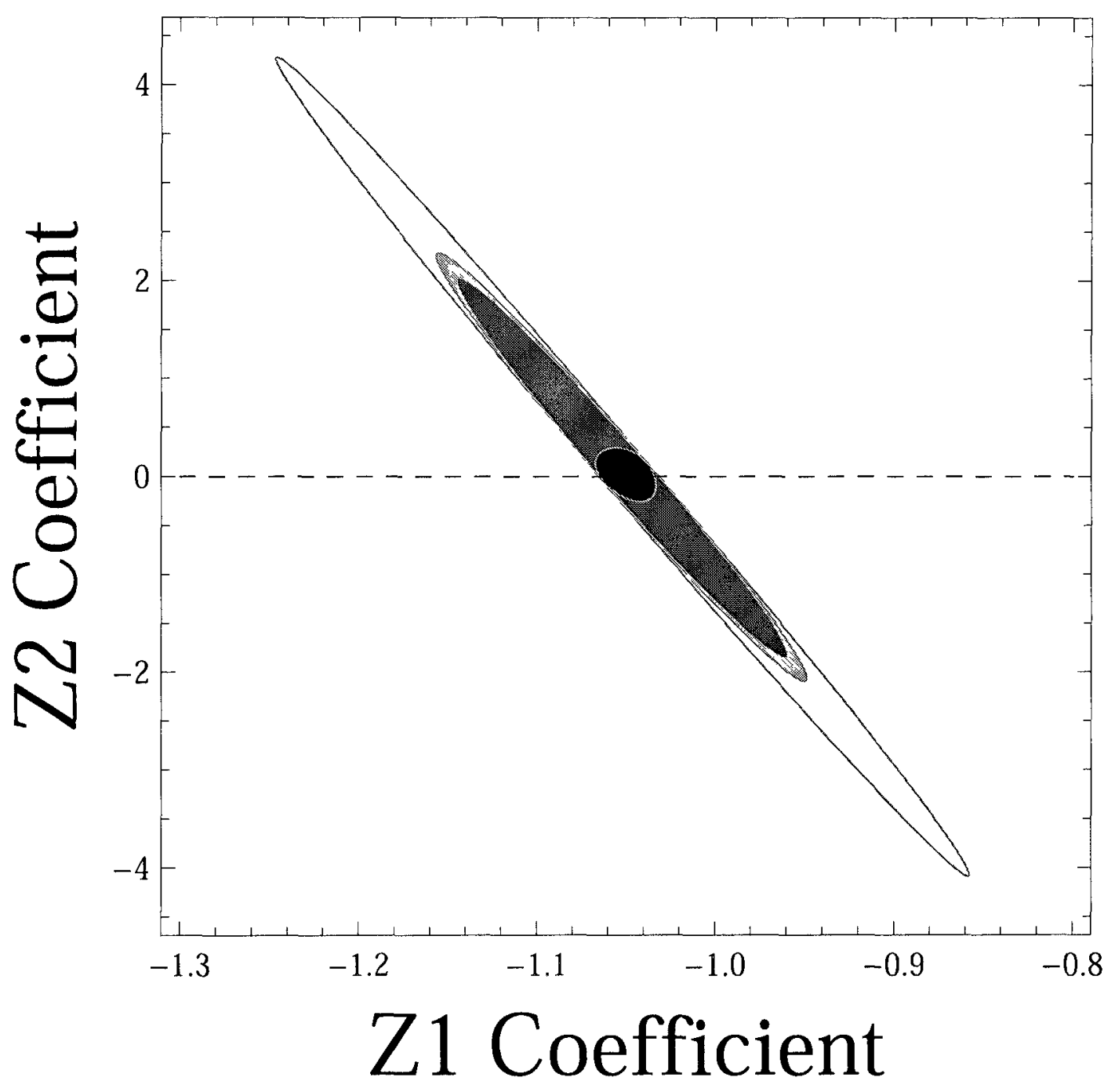

Figure 4.17: Extraction of $Z^{(1)}$ and $Z^{(2)}$ couplings from a $\chi^{2}$ at 95 percent confidence level. The data points used, from the outside in, are: 0.5 and $3 \mathrm{TeV} ; 1$ and $3 \mathrm{TeV}$; $0.5,1$, and $3 \mathrm{TeV} ; 0.5,1,3$ and $5 \mathrm{TeV}$. Here we have set the width of $Z^{(2)}$ equal to its mass, with $M_{1}=2.2$. 


\section{Chapter 5}

\section{Conclusions}

\subsection{Discussion and Conclusions}

In this thesis we studied the Higgsstrahlung cross section in the Minimal Composite Higgs Model at center-of-mass energies spanning the masses of the first several KK gauge excitations. We have calculated the MCHM Higgsstrahlung cross section both with and without including full particle mixing. The MCHM cross section exhibits a strong suppression in comparison with that of SM Higgsstrahlung. This suppression is primarily due to a systematic cancellation between the contributions of different Kaluza-Klein modes propagating through the interaction. In fact, the extent of the suppression is largely determined by both the magnitude and sign of the first $Z \mathrm{KK}$ mode coupling. As it is both slightly larger in magnitude and opposite in sign to the zero-mode coupling, it provides the dominant reduction of MCHM cross section; the cancellation between the two reduces the magnitude of the cross section by roughly 90 percent. The inclusion or omission of full particle mixing does not substantially alter this suppression; the relevant couplings become a few percent smaller across the 
board, but the behaviour of the coupling cancellations remains the same

The suppression between resonances displays an energy dependence which can be fitted to the form $y=x^{a} \exp \left[b+c(\ln x)^{2}\right]$ Although this form suggests an exponential influence on the dependence, it is evidently not a simple exponential suppression, as one might anticipate from the suppression in the Coleman-Weinberg potential, the exponential behaviour is diminished both by a logarithmic function in its argument and by a multiplicative power law factor Furthermore, the alternating coupling sum sequence is slightly offset from zero, suggesting that as the energy increases the suppression will deviate from the fit function behaviour entirely and approach a constant, rather than approach zero as a true exponential or power law suppression would However, this offset is very small, and would still allow a suppression of at least seven or elght orders of magnitude when compared with the SM In the end, although there are certain resemblances between the suppressions observed in the Higgsstralung cross section and the Coleman-Weinberg potentıal, the mechanısms behind each are obviously not completely analogous The exact relationship between the two remauns unclear

A secondary suppression in the cross section is caused by the extremely large widths of the KK bosons However, this effect is not easily quantifiable as width calculations for the higher KK modes become invalid when full particle mixing is incorporated, this is the primary effect of including the full mixing, as it significantly alters the couplings of heavy KK modes Due to the influence of strong couplings between these modes, the widths become larger than the associated particle mass, Indicating a breakdown of the perturbation theory

We also performed a brief analysis of the feasibility of extracting the couplings of this model at electron-positron colliders such as the proposed ILC and CLIC We 
determined that the MCHM zero-mode Higgsstrahlung coulping should be clearly distinguishable from the SM coupling at both colliders Furthermore, with the extended energy range of CLIC the magnitude and sign of the first KK mode coupling - the key characteristics which lead to the primary cross-section suppression - should also be identifiable Furthernore, while the second KK coupling will not be differentiable from zero, CLIC would be able to constrain its magnitude, requiring that it be less than a fourth of the first KK mode's magnitude, this would provide fur ther cvidence of the suppression, as the couplings must both become increasingly smaller in size and alternate in sign for the effect to occur

\subsection{Future Research}

There are several avenues open for future research The first is to develop a more rigorous relation between the suppression behaviour seen in both the Higgsstrahlung cross section and the Coleman-Weinberg potential Although we have a strong mathematical understanding of the origin and exponentıal nature of the Coleman-Weinberg suppression, we have yet to achieve the same for the Higgsstrahlung interaction (our results are only numerical), or to systematically relate the two This question bears further thought and investigation Further work in this direction could involve delving deeper into the mathematics analytically or studying other Higgs interactions to determine whether or not similar suppressions arise

A second option is to extend the current work by performing a detaıled collider study of the Higgsstrahlung interaction at the ILC and CLIC, with the goal of both confirming and extending the introductory phenomenology presented in sections 435 and 442 Furthermore, a similar analysis - both theoretical and phenomenological - 
to that presented in this thesis could be performed for Higgsstrahlung from a proton proton collision. Such an analysis would be more complicated due to the need to incorporate the parton distribution functions, but would yield results relevant to the Large Hadron Collider currently in operation at CERN in Geneva.

Finally, a third option is to attempt to develop methods of incorporating both widths and particle mixing - and by extension zero-mode masses - into the 5D propagator. If such methods were to be developed, the full mixing cross section could be calculated in both the EVA and ECA approaches. This might lend a different perspective to the mechanisms behind the coupling and cross-section behaviours of the Higgsstrahlung interaction. 


\section{Appendix A}

\section{Special Functions}

\section{A.1 Bessel Functions}

Bessel functions of the first and second kind $-J_{n}(x)$ and $Y_{n}(x)$ respectively - are solutions to Bessel's differential equation [61, 62, 63],

$$
x^{2} \frac{d^{2} y}{d x^{2}}+x \frac{d y}{d x}+\left(x^{2}-n^{2}\right) y=0
$$

where $n$ is a non-negative real number. The two solutions are independent only if $n$ is not an integer; otherwise they are related through

$$
Y_{n}(z)=\frac{J_{n}(z) \cos (n \pi)-J_{-n}(z)}{\sin (n \pi)}
$$

The Bessel functions are orthogonal according to

$$
\int_{0}^{a} J_{\nu}\left(\alpha_{n} \frac{\rho}{a}\right) J_{\nu}\left(\alpha_{m} \frac{\rho}{a}\right) \rho d \rho=\frac{1}{2} a^{2}\left[J_{\nu+1}\left(\alpha_{m}\right)\right]^{2} \delta_{m n}
$$

where $\alpha_{m}$ is the $m$ th zero of $J_{\nu}$, and similarly for $Y_{\nu}$. Their asymptotic approximations 
are

$$
\begin{aligned}
& J_{n}(x) \approx \begin{cases}\frac{1}{\Gamma(n+1)}\left(\frac{z}{2}\right)^{n} & \text { for } x \ll 1 \\
\sqrt{\frac{2}{\pi x}} \cos \left(x-\frac{n \pi}{2}-\frac{\pi}{4}\right) & \text { for } x \gg\left|n^{2}-\frac{1}{4}\right|\end{cases} \\
& Y_{n}(x) \approx \begin{cases}\frac{2}{\pi}\left[\ln \left(\frac{1}{2} x\right)+\gamma\right] & \text { for } m=0, x \ll 1 \\
\frac{\Gamma(n)}{\pi}\left(\frac{2}{x}\right)^{n} & \text { for } m \neq 0, x \ll 1 \\
\sqrt{\frac{2}{\pi x}} \sin \left(x-\frac{n \pi}{2}-\frac{\pi}{4}\right) & \text { for } x \gg 1\end{cases}
\end{aligned}
$$

and they obey the recurrence relations

$$
\begin{gathered}
J_{n+1}(x)=\frac{2 n}{x} J_{n}(x)-J_{n-1}(x) \\
\frac{d}{d x} J_{n}(x)=\frac{1}{2}\left[J_{n-1}(x)-J_{n+1}(x)\right]=J_{n-1}(x)-\frac{n}{x} J_{n}(x)=\frac{n}{x} J_{n}(x)-J_{n+1}(x) \\
\frac{d}{d x}\left[x^{m} J_{m}(x)\right]=x^{m} J_{m-1}(x)
\end{gathered}
$$

and similarly for $Y_{n}(x)$

The modified Bessel functions of the first and second kind $-I_{n}(x)$ and $K_{n}(x)$ - are solutions to $\mathrm{Eq}$ (A 1) under the transformation $x \rightarrow \imath x[61,62,63]$ The Bessel functions of the first kind are related through

$$
I_{n}(x)=\imath^{-n} J_{n}(\imath x)
$$

In the case where $n$ is an integer, the Bessel functions of the second kind are related as follows

$$
\begin{aligned}
K_{n}(x) & =\frac{\pi}{2} \frac{I_{-n}(x)-I_{n}(x)}{\sin (n \pi)}=\frac{\pi}{2} \frac{\imath^{n} J_{-n}(\imath x)-\imath^{-n} J_{n}(\imath x)}{\sin (n \pi)} \\
& =-\imath^{n} \frac{\pi}{2} \frac{J_{n}(\imath x) \cos (n \pi)-J_{n}(\imath x)}{\sin (n \pi)}=-\imath^{n} \frac{\pi}{2} Y_{n}(\imath x)
\end{aligned}
$$

The modified Bessel functions obey the same recurrence relations as the orig- 
inal Bessel function, and have the following asymptotic approximations,

$$
\begin{aligned}
& I_{n}(x) \approx \begin{cases}\frac{1}{n^{\prime}}\left(\frac{x}{2}\right)^{n} & \text { for } x \ll 1 \\
\frac{\exp (x)}{\sqrt{2 \pi x}} & \text { for } x \gg 1\end{cases} \\
& K_{n}(x) \approx \begin{cases}\sqrt{\frac{\pi}{2 x}} \exp (-x) & \text { for } x \gg 1 \\
-\ln \left(\frac{x}{2}\right)-\gamma & \text { for } x \ll 1, n=0 \\
\frac{\Gamma(n)}{2}\left(\frac{2}{x}\right)^{n} & \text { for } x \ll 1, n \neq 0,\end{cases}
\end{aligned}
$$

where $\gamma=05772$ is the Euler-Mascheron constant

\section{A.2 Meijer G Functions}

The Mejjer $G$ function $M_{G}\left(a_{s}, b_{s}, c_{s}, d_{s}, z\right)$ is defined as a complex countour integral over a number of Gamma functions $[62,63]$ Its first four arguments are lists of numbers

$$
\begin{aligned}
a_{s} & =\left[\begin{array}{ll}
a_{1}, a_{2} & a_{k}
\end{array}\right] \\
b_{s} & =\left[\begin{array}{ll}
b_{1}, b_{2} & b_{m}
\end{array}\right] \\
c_{s} & =\left[\begin{array}{ll}
c_{1}, c_{2} & c_{n}
\end{array}\right] \\
d_{s} & =\left[\begin{array}{ll}
d_{1}, d_{2} & d_{l}
\end{array}\right]
\end{aligned}
$$

and it is defined as

$$
M_{G}\left(a_{s}, b_{s}, c_{s}, d_{s}, z\right)=\frac{1}{2 \pi \imath} \oint_{\gamma_{L}} d y z^{y} \frac{\left[\prod_{s=1}^{k} \Gamma\left(1-a_{s}+y\right)\right]\left[\prod_{s=1}^{n} \Gamma\left(c_{s}-y\right)\right]}{\left[\prod_{s=1}^{m} \Gamma\left(b_{s}+y\right)\right]\left[\prod_{s=1}^{l} \Gamma\left(1-d_{s}+y\right)\right]}
$$




\section{A.3 The Generalized Hypergeometric Function}

The generalized hypergeometric function is defined as $[62,63]$

$$
F\left(a_{s} ; b_{s} ; z\right)=\sum_{n=0}^{\infty} \frac{z^{n}}{n !} \prod_{\imath=1}^{k} \prod_{\jmath=1}^{m} \frac{\Gamma\left(a_{\imath}+n\right)}{\Gamma\left(a_{\imath}\right)} \frac{\Gamma\left(b_{\jmath}\right)}{\Gamma\left(b_{\jmath}+n\right)}
$$

The lists $a_{s}$ and $b_{s}$ are defined as in Eq. (A.14).

\section{A.4 Lommel Functions}

The first Lommel function $L_{1}(a, b, z)$ is defined in terms of the generalized hypergeometric function $F\left(a_{s} ; b_{s} ; z\right)$ of Eq. (A.16) as $[62,63]$

$L_{1}(a, b, z)=\frac{z^{a+1}}{(a-b+1)(a+b+1)} F\left(1 ; \frac{3}{2}-\frac{1}{2}(b-a), \frac{3}{2}+\frac{1}{2}(b+a) ;-\frac{z^{2}}{4}\right)$.

\section{A.5 Struve Functions}

The Struve function $H_{\nu}(z)$ is defined in terms of Gamma functions as $[62,63]$

$$
H_{\nu}(z)=\left(\frac{1}{2 z}\right)^{\nu+1} \sum_{k=0}^{\infty} \frac{(-1)^{k}(2 z)^{-2 k}}{\Gamma(k+3 / 2) \Gamma(k+\nu+3 / 2)} .
$$

\section{A.6 The Confluent Hypergeometric Functions}

The confluent hypergeometric functions of the first and sccond kinds, $K(\alpha, \gamma, z)$ and $W(\alpha, \gamma, z)$, are the two solutions to the confluent hypergeometric differential equation $[62,63]$

$$
z \frac{d^{2}}{d z^{2}} y(z)+(\gamma-z) \frac{d}{d z} y(z)-\alpha y(z)=0
$$

They are sometimes known as Kummel functions. 


\section{Appendix B}

\section{Generators}

\section{B.1 Definition}

In the 5-representation, the generators of $\mathrm{SO}(4) \approx \mathrm{SU}(2)_{L} \times \mathrm{SU}(2)_{R}$ are

$$
T_{\imath \jmath}^{a_{L, R}}=-\frac{\imath}{2}\left[\frac{1}{2} \epsilon^{a b c}\left(\delta_{\imath}^{b} \delta_{\jmath}^{c}-\delta_{\jmath}^{b} \delta_{\imath}^{c}\right) \pm\left(\delta_{\imath}^{a} \delta_{\jmath}^{4}-\delta_{\jmath}^{a} \delta_{\imath}^{4}\right)\right]
$$

while the generators for $\mathrm{SO}(5) / \mathrm{SO}(4)$ are

$$
T_{\imath \jmath}^{\hat{a}}=-\frac{1}{\sqrt{2}}\left(\delta_{\imath}^{\hat{a}} \delta_{\jmath}^{5}-\delta_{\jmath}^{\hat{a}} \delta_{5}^{a}\right)
$$

where $a_{L, R}=1,2,3, \hat{a}=1 \ldots 4$ and $\imath, \jmath=1 \ldots 5[3]$. Explicitely, the generators are given by

$$
T^{1_{L, R}}=-\frac{\imath}{2}\left(\begin{array}{ccccc}
0 & 0 & 0 & \pm 1 & 0 \\
0 & 0 & 1 & 0 & 0 \\
0 & -1 & 0 & 0 & 0 \\
\mp 1 & 0 & 0 & 0 & 0 \\
0 & 0 & 0 & 0 & 0
\end{array}\right) \quad T^{2_{L, R}}=-\frac{\imath}{2}\left(\begin{array}{ccccc}
0 & 0 & -1 & 0 & 0 \\
0 & 0 & 0 & \pm 1 & 0 \\
1 & 0 & 0 & 0 & 0 \\
0 & \mp 1 & 0 & 0 & 0 \\
0 & 0 & 0 & 0 & 0
\end{array}\right)
$$




$$
\begin{aligned}
& T^{3_{L} R}=-\frac{\imath}{2}\left(\begin{array}{ccccc}
0 & 1 & 0 & 0 & 0 \\
-1 & 0 & 0 & 0 & 0 \\
0 & 0 & 0 & \pm 1 & 0 \\
0 & 0 & \mp 1 & 0 & 0 \\
0 & 0 & 0 & 0 & 0
\end{array}\right) \quad T^{\hat{1}}=-\frac{\imath}{\sqrt{2}}\left(\begin{array}{ccccc}
0 & 0 & 0 & 0 & 1 \\
0 & 0 & 0 & 0 & 0 \\
0 & 0 & 0 & 0 & 0 \\
0 & 0 & 0 & 0 & 0 \\
-1 & 0 & 0 & 0 & 0
\end{array}\right) \\
& T^{2}=-\frac{\imath}{\sqrt{2}}\left(\begin{array}{ccccc}
0 & 0 & 0 & 0 & 0 \\
0 & 0 & 0 & 0 & 1 \\
0 & 0 & 0 & 0 & 0 \\
0 & 0 & 0 & 0 & 0 \\
0 & -1 & 0 & 0 & 0
\end{array}\right) \quad T^{\hat{3}}=-\frac{\imath}{\sqrt{2}}\left(\begin{array}{ccccc}
0 & 0 & 0 & 0 & 0 \\
0 & 0 & 0 & 0 & 0 \\
0 & 0 & 0 & 0 & 1 \\
0 & 0 & 0 & 0 & 0 \\
0 & 0 & -1 & 0 & 0
\end{array}\right) \\
& T^{\hat{4}}=-\frac{\imath}{\sqrt{2}}\left(\begin{array}{ccccc}
0 & 0 & 0 & 0 & 0 \\
0 & 0 & 0 & 0 & 0 \\
0 & 0 & 0 & 0 & 0 \\
0 & 0 & 0 & 0 & 1 \\
0 & 0 & 0 & -1 & 0
\end{array}\right)
\end{aligned}
$$

In the 10-representation, or adjoint representation, $T^{\hat{4}}, T^{\hat{3}}$ and $T^{3_{L} R}$ are

$$
T_{10}^{\hat{4}}=\frac{\imath}{2}\left(\begin{array}{cccccccccc}
0 & 0 & 0 & 0 & 1 & 0 & 0 & -1 & 0 & 0 \\
0 & 0 & 0 & 0 & 0 & 1 & 0 & 0 & -1 & 0 \\
0 & 0 & 0 & 0 & 0 & 0 & 1 & 0 & 0 & -1 \\
0 & 0 & 0 & 0 & 0 & 0 & 0 & 0 & 0 & 0 \\
-1 & 0 & 0 & 0 & 0 & 0 & 0 & 0 & 0 & 0 \\
0 & -1 & 0 & 0 & 0 & 0 & 0 & 0 & 0 & 0 \\
0 & 0 & -1 & 0 & 0 & 0 & 0 & 0 & 0 & 0 \\
1 & 0 & 0 & 0 & 0 & 0 & 0 & 0 & 0 & 0 \\
0 & 1 & 0 & 0 & 0 & 0 & 0 & 0 & 0 & 0 \\
0 & 0 & 1 & 0 & 0 & 0 & 0 & 0 & 0 & 0
\end{array}\right) \quad T_{10}^{3}=\frac{\imath}{2}\left(\begin{array}{ccccccccccc}
0 & 0 & 0 & 0 & 0 & 1 & 0 & 0 & 1 & 0 \\
0 & 0 & 0 & 0 & -1 & 0 & 0 & -1 & 0 & 0 \\
0 & 0 & 0 & 0 & 0 & 0 & 0 & 0 & 0 & 0 \\
0 & 0 & 0 & 0 & 0 & 0 & 1 & 0 & 0 & -1 \\
0 & -1 & 0 & 0 & 0 & 0 & 0 & 0 & 0 & 0 \\
1 & 0 & 0 & 0 & 0 & 0 & 0 & 0 & 0 & 0 \\
0 & 0 & 0 & 1 & 0 & 0 & 0 & 0 & 0 & 0 \\
0 & -1 & 0 & 0 & 0 & 0 & 0 & 0 & 0 & 0 \\
1 & 0 & 0 & 0 & 0 & 0 & 0 & 0 & 0 & 0 \\
0 & 0 & 0 & -1 & 0 & 0 & 0 & 0 & 0 & 0 \\
0 &
\end{array}\right.
$$




\section{B.2 Commutation relations}

The generators obey the following commutation relations:

$$
\begin{aligned}
{\left[T^{a_{L, R}}, T^{b_{L, R}}\right] } & =i \epsilon^{a b c} T^{c_{L, R}}, \quad\left[T^{a_{L}}, T^{b_{R}}\right]=0, \\
{\left[T^{\hat{a}}, T^{\hat{b}}\right] } & =\frac{1}{2}\left[\delta_{i}^{\hat{a}} \delta_{j}^{\hat{b}}-\delta_{j}^{\hat{a}} \delta_{\imath}^{\hat{b}}\right]=\frac{i}{2}\left[\epsilon^{a b c}\left(T^{c_{L}}+T^{c_{R}}\right)+\delta_{b}^{4}\left(T^{a_{L}}-T^{a_{R}}\right)\right], \\
{\left[T^{a_{L}, R}, T^{\hat{b}}\right] } & =\frac{i}{2}\left(\epsilon^{a b c} T^{\hat{c}} \mp T^{\hat{a}} \delta_{b}^{4} \pm T^{\hat{4}} \delta_{b}^{a}\right),
\end{aligned}
$$

where $\epsilon^{a b c}$ is the totally antisymmetric tensor with $\epsilon^{123}=1$.

\section{B.3 Expansions of $A_{M N}^{a}$}

Using these generators, our defined quantities $A_{M N}^{a} T^{a}=-i\left[T^{c}, T^{b}\right] A_{M}^{c} A_{N}^{b}$ become, where $a b c$ is an even permutation of 123 ,

$$
\begin{aligned}
A_{M N}^{a_{L}}= & A_{M}^{b_{L}} A_{N}^{c_{L}}-A_{M}^{c_{L}} A_{N}^{b_{L}}+\frac{1}{2}\left(A_{M}^{\hat{b}} A_{N}^{\hat{c}}-A_{M}^{\hat{c}} A_{N}^{\hat{b}}+A_{M}^{\hat{a}} A_{N}^{\hat{4}}-A_{M}^{\hat{4}} A_{N}^{\hat{a}}\right) \\
A_{M N}^{a_{R}}= & A_{M}^{b_{R}} A_{N}^{c_{R}}-A_{M}^{c_{R}} A_{N}^{b_{R}}+\frac{1}{2}\left(A_{M}^{\hat{b}} A_{N}^{\hat{c}}-A_{M}^{\hat{c}} A_{N}^{\hat{b}}-A_{M}^{\hat{a}} A_{N}^{\hat{4}}+A_{M}^{\hat{4}} A_{N}^{\hat{a}}\right) \\
A_{M N}^{\hat{a}}= & \frac{1}{2}\left[\left(A_{M}^{b_{L}}+A_{M}^{b_{R}}\right) A_{N}^{\hat{c}}-A_{M}^{\hat{c}}\left(A_{N}^{b_{L}}+A_{N}^{b_{R}}\right)-\left(A_{M}^{c_{L}}+A_{M}^{c_{R}}\right) A_{N}^{\hat{b}}\right. \\
\quad & \left.A_{M}^{\hat{b}}\left(A_{N}^{c_{L}}+A_{N}^{c_{R}}\right)-\left(A_{M}^{a_{L}}-A_{M}^{a_{R}}\right) A_{N}^{\hat{4}}+A_{M}^{\hat{4}}\left(A_{N}^{a_{L}}-A_{N}^{a_{R}}\right)\right] .
\end{aligned}
$$

$A_{M N}^{\hat{4}}$ is given by

$$
A_{M N}^{\hat{4}}=\frac{1}{2} \sum_{a=1}^{3}\left[\left(A_{M}^{a_{L}}-A_{M}^{a_{R}}\right) A_{N}^{\hat{a}}-A_{M}^{\hat{a}}\left(A_{N}^{a_{L}}-A_{N}^{a_{R}}\right)\right],
$$




\section{Appendix $\mathrm{C}$}

\section{Derivation of Feynman Rules}

\section{C.1 Propagators}

The relevant part of the gauge Lagrangian, Eq. (3.8), for the gauge boson propagator is

$$
L_{\text {prop }}=\sqrt{\bar{g}}\left[-\frac{1}{4} g^{M N} g^{R S}\left(\partial_{M} A_{R}^{a}-\partial_{R} A_{M}^{a}\right)\left(\partial_{N} A_{S}^{a}-\partial_{S} A_{N}^{a}\right)+\frac{1}{2} m^{2} k^{2} g^{M N} A_{M}^{a} A_{N}^{a}+L_{G F}\right],
$$

where $\bar{g}$ is the determinant of the metric. The gauge fixing term is chosen to be [46, 38]

$$
\sqrt{\bar{g}} L_{G F}=-\frac{1}{2 \epsilon k z}\left[\partial^{\mu} A_{\mu}^{a}-\epsilon z \partial_{z}\left(\frac{1}{z} A_{5}^{a}\right)\right]^{2} .
$$

In the following derivations of propagators, we assume that EWSB has not yet occured, and omit any mass terms or mixing which might occur due to the Higgs vacuum expectation value. 


\section{C.1.1 Gauge Boson $A_{\mu}$}

We find that the propagator for a gauge boson $A_{\mu}^{a}, \mu=0 \quad 3$ with 4D momentum $p^{\mu}$, moving between two points $z$ and $z^{\prime}$ in the fifth dimension and with boundary mass $m$, 1s given by

$$
\begin{array}{lr}
-\imath G_{m}\left(z, z^{\prime}, p\right)\left(\eta^{\nu \mu}-\frac{p^{\mu} p^{\nu}}{p^{2}}\right)-\imath G_{m}\left(z, z^{\prime}, \frac{p}{\sqrt{\epsilon}}\right)\left(\frac{p^{\mu} p^{\nu}}{p^{2}}\right), & \text { in general, } \\
-\imath \eta_{\mu \nu} G_{m}\left(z, z^{\prime}, p\right) & \epsilon=1, \\
-\imath G_{m}\left(z, z^{\prime}, p\right)\left(\eta^{\nu \mu}-\frac{p^{\mu} p^{\nu}}{p^{2}}\right)-\imath G_{m}\left(z, z^{\prime}, 0\right)\left(\frac{p^{\mu} p^{\nu}}{p^{2}}\right), & \epsilon=\infty, \\
-\imath G_{m}\left(z, z^{\prime}, p\right)\left(\eta_{\mu \nu}+\frac{p^{\mu} p^{\nu}}{p^{2}}\right) & \epsilon=0,
\end{array}
$$

in general, Feynman gauge, unitary gauge, and Lorentz gauge respectively [38] The Green's function $G_{m}\left(z, z^{\prime}, p\right)$ is defined by

$$
\left[p^{2}-\frac{1}{z} \partial_{z}+\partial_{z}^{2}-\frac{m^{2}}{z^{2}}\right] G_{m}\left(z, z^{\prime}, p\right)=k z \delta\left(z-z^{\prime}\right)
$$

The solution to Eq (C 6) is given by

$$
G_{m}(u, v, p)=\frac{\pi}{2} \frac{k u v}{A D-B C}\left[A J_{\alpha}(p u)+B Y_{\alpha}(p u)\right]\left[C J_{\alpha}(p v)+D Y_{\alpha}(p v)\right]
$$

where $\alpha=\sqrt{1+m^{2}}, u=\min \left(z, z^{\prime}\right), v=\max \left(z, z^{\prime}\right)$ and $A, B, C, D$ wlll be determined by BCs ${ }^{1}$ With Neumann-Neumann boundary conditions - which all SM particles will have, as they must be even under $Z_{2}$ symmetry - given by

$$
\partial_{u} G\left(L_{0}, v, p\right)=\partial_{v} G\left(u, L_{1}, p\right)=0
$$

we find that $A=-Y_{\alpha-1}\left(p L_{0}\right), B=J_{\alpha-1}\left(p L_{0}\right), C=-Y_{\alpha-1}\left(p L_{1}\right)$, and $D=J_{\alpha-1}\left(p L_{1}\right)$ Similarly, with Dirıchlet-Neumann boundary conditions, given by

$$
G\left(L_{0}, v, p\right)=\partial_{v} G\left(u, L_{1}, p\right)=0
$$

\footnotetext{
${ }^{1}$ As we will be neglecting brane mass terms, for our purposes $m=0$
} 
we have $A=-Y_{\alpha}\left(p L_{0}\right), B=J_{\alpha}\left(p L_{0}\right), C=-Y_{a-1}\left(p L_{1}\right)$, and $D=J_{\alpha-1}\left(p L_{1}\right)$

The third relevant set of boundary conditions for our purposes is the DirichletDirichlet condition,

$$
G\left(L_{0}, v, p\right)=G\left(u, L_{1}, p\right)=0
$$

which y1elds $A=-Y_{\alpha}\left(p L_{0}\right), B=J_{\alpha}\left(p L_{0}\right), C=-Y_{\alpha}\left(p L_{1}\right)$, and $D=J_{\alpha}\left(p L_{1}\right)$ However, as the $A_{\mu}^{a}$ do not couple to fermion anti-fermion pairs, this propagator is not relevant for our calculations

\section{C.1.2 Fifth Component $A_{5}^{a}$}

We find that the propagator for a fifth component $A_{5}^{a}$ moving between two points $z$ and $z^{\prime}$ is given by

$$
\begin{array}{lr}
\frac{\imath}{\epsilon} G_{\sqrt{m^{2}-1}}\left(z, z^{\prime}, \frac{p}{\sqrt{c}}\right), & \text { in general } \\
-\imath G_{\sqrt{m^{2}-1}}\left(z, z^{\prime}, p\right) & \text { in Feynman gauge } \epsilon=1, \\
0, & \text { in unitary gauge } \epsilon=\infty,
\end{array}
$$

where $G_{m}\left(z, z^{\prime}, p\right)$ is defined by Eq (C 6 ) and given cxplicitly by Eq (C 7) [38] The unitary result is due to the fact that in the unitary gauge $A_{5}^{a}=0$ However, note that for the $A_{5}^{a}$ the gauge symmetry is broken by the BCs, and we cannot just choose the gauge of our choice, therefore $A_{5}^{a} \neq 0$, and this definition of the propagator is not compatible 


\section{C.2 Profiles without particle mixing}

In the following derivations of profiles, we omit the effects of EWSB; profiles including these effects will be addressed in Sec. C.3.

\section{C.2.1 Boson Profiles}

We will need the 5-dimensional profiles for the $Z$ and $X \mathrm{KK}$ gauge bosons, which correspond to the $\mathrm{n}^{\text {th }} 5 \mathrm{D}$ component $f_{G}^{(n)}(z)$ of the Kaluza-Klein (KK) decomposition, ${ }^{2}$

$$
A_{\mu}(x, z)=\sum_{n} A_{\mu}^{(n)}(x) f_{G}^{(n)}(z) .
$$

Using the Lagrangian of Eq. (3.8) and the principle of least action, it can be shown that $f_{G}^{(n)}(z)$ must satisfy [51]

$$
\left[\partial_{z}^{2}-\frac{1}{z} \partial_{z}+m_{n}^{2}\right] f_{G}^{(n)}(z)=0
$$

This differential equation has the solution

$$
f_{G}^{(n)}(z)=A_{n} k z\left[J_{1}\left(m_{n} z\right)+B_{n} Y_{1}\left(m_{n} z\right)\right]
$$

where the constants are defined by the BCs and the orthonomalization condition [51, 39]. The normalization condition can be derived by comparing the $5 \mathrm{D}$ theory to the 4D Standard Model (SM); if we integrate the kinetic terms of the 5D Lagrangian, we should obtain the appropriate kinetic terms of the 4D Lagrangian. Consider the

\footnotetext{
${ }^{2}$ Here $A_{\mu}$ denotes a generic boson; the $A_{\mu}^{a}$ and $U_{\mu}$ boson profiles have the same form and derivation.
} 
kınetic term of two zero mode boson fields,

$$
\begin{aligned}
L_{4 \mathrm{eff}} & =\int_{L_{0}}^{L_{1}} d z L_{5 \mathrm{k} 1 \mathrm{n}}=\int_{L_{0}}^{L_{1}} d z \frac{1}{2 k z} \partial_{\mu} A_{\nu}^{(0)}(x, z) \partial^{\mu} A^{(0) \nu}(x, z) \\
& =\frac{1}{2} \partial_{\mu} \tilde{A}_{\nu}^{(0)}(x) \partial^{\prime \prime} \tilde{A}^{(0) \nu}(x) \int_{L_{0}}^{L_{1}} d z \frac{1}{k z} f_{G}^{(0)}(z) f_{G}^{(0)}(z)
\end{aligned}
$$

where $\tilde{A}_{\nu}^{(0)}(x, z)$ denotes the 4-dimensional SM field We can see that for $L_{4 \text { eff }}$ to correspond to the SM, the integral in the last line must evaluate to one Accounting for the orthogonality of different modes, we obtain the following orthonormalization condition

$$
\int_{L_{0}}^{L_{1}} d z \frac{1}{k z} f_{G}^{(m)}(z) f_{G}^{(n)}(z)=\delta_{m n}
$$

It is convenient to define the following basis of solutions that are even (Neumann BC) or odd (Dirıchlet BC) on the Planck brane,

$$
\begin{aligned}
& C_{A}\left(m_{n}, z\right)=\frac{\pi m_{n}}{2} z\left[J_{1}\left(m_{n} z\right) Y_{0}\left(m_{n} L_{0}\right)-J_{0}\left(m_{n} L_{0}\right) Y_{1}\left(m_{n} z\right)\right] \\
& S_{A}\left(m_{n}, z\right)=\frac{\pi m_{n}}{2} z\left[J_{1}\left(m_{n} L_{0}\right) Y_{1}\left(m_{n} z\right)-J_{1}\left(m_{n} z\right) Y_{1}\left(m_{n} L_{0}\right)\right]
\end{aligned}
$$

where $C_{A}\left(m_{n}, L_{0}\right)=1, \partial_{z} C_{A}\left(m_{n}, L_{0}\right)=0, S_{A}\left(m_{n}, L_{0}\right)=0$ and $\partial_{z} S_{A}\left(m_{n}, L_{0}\right)=m_{n}$ Then the profiles of bosons with Neumann-Neumann (NN), Dirıchlet-Neumann (DN) and Dirıchlet-Dirıchlet (DD) boundary conditions are given by

$$
f_{G}^{(n)}= \begin{cases}C_{G}^{(n)} C_{A}\left(m_{n}, z\right) & \text { for NN BCs } \\ C_{G}^{(n)} S_{A}\left(m_{n}, z\right) & \text { for DN and DD BCs }\end{cases}
$$

where the coefficients $C_{G}$ are fixed using $\mathrm{Eq}$ (C 18), and $G$ denotes the gauge boson in question ${ }^{3}$ By applying the appropriate boundary condition to these profiles on

\footnotetext{
${ }^{3}$ There are no gauge bosons in the MCHM with Neumann-Dirıchlet BCs
} 
the $\mathrm{TeV}$ brane, we obtain conditions that define the boson masses in the non-mixing limit,

$$
\begin{array}{cc}
Y_{0}\left(p L_{0}\right) J_{0}\left(p L_{1}\right)-J_{0}\left(p L_{0}\right) Y_{0}\left(p L_{1}\right)=0 & \text { for NN BCs } \\
Y_{1}\left(p L_{0}\right) J_{0}\left(p L_{1}\right)-J_{1}\left(p L_{0}\right) Y_{0}\left(p L_{1}\right)=0 & \text { for DN BCs } \\
Y_{1}\left(p L_{0}\right) J_{1}\left(p L_{1}\right)-J_{1}\left(p L_{0}\right) Y_{1}\left(p L_{1}\right)=0 & \text { for DD BCs }
\end{array}
$$

where we have used the relations in Eq (A 8) to evaluate the derıvatıves

The zero mode gauge boson profile can be found by solving $\mathrm{Eq}$ (C 15) with $m_{0}=0$ Takıng Neumanı BCs, $\partial_{z} f_{G}^{(0)}\left(z=L_{0}, L_{1}\right)=0$ - where brane kinetıc terms have been neglected - the solution has the form $f_{G}^{(0)}(z)=$ constant Using the orthonormality condition we find that,

$$
f_{G}^{(0)}(z)=\sqrt{\frac{k}{\ln \left(k L_{1}\right)}}
$$

\section{C.2.2 Fermion Profiles}

The Lagrangian for a fermion is given by $\mathrm{Eq}$ (3 12) As mentioned in the text, the spin connection $w_{M A B}$ cancels out, and the relevant part of the covariant derivitive for the profile is simply the normal derıvative $\partial_{M}$ We therefore have

$$
\begin{aligned}
L_{f} & =\sqrt{g}\left[\frac{\imath}{2} \bar{\Psi}(k z) \Gamma^{M} \partial_{M} \Psi-\frac{\imath}{2}\left(\partial_{M} \Psi\right)^{\dagger} \Gamma^{0}(k z) \Gamma^{M} \Psi-M_{\Psi} \bar{\Psi} \Psi\right] \\
& =\bar{\Psi}\left[\imath \sqrt{g}(k z) \Gamma^{M} \partial_{M}+\frac{\imath}{2} \Gamma^{M} \partial_{M}(\sqrt{g}(k z))-\sqrt{g} M_{\Psi}\right] \Psi \\
& =\bar{\Psi}\left[\imath \sqrt{g}(k z) \gamma^{\mu} \partial_{\mu}+\sqrt{g}(k z) \gamma^{\nu} \partial_{5}+\frac{1}{2} \gamma^{5} \partial_{5}(\sqrt{g}(k z))-\sqrt{g} M_{\Psi}\right] \Psi \\
& =\frac{1}{(k z)^{4}} \bar{\Psi}\left[\not p+\gamma^{5} \partial_{5}-\frac{1}{k z}\left(2 k \gamma^{5}+M_{\Psi}\right)\right] \Psi,
\end{aligned}
$$

where we have applied integration by parts on the second term in the second line The fermion fields can be bioken into their chiral components $\Psi=\Psi_{L}+\Psi_{R}$ where 
$\gamma_{5} \Psi_{L R}=\mp \Psi_{L R} \quad$ From Eq (C 29) it can then be shown that the left- and righthanded fermions are related through

$$
\begin{gathered}
{\left[\partial_{z}-z^{-1}(2-c)\right] \Psi_{L}^{(n)}(z)=-m_{n} \Psi_{R}^{(n)}} \\
{\left[\partial_{z}-z^{-1}(2+c)\right] \Psi_{R}^{(n)}(z)=m_{n} \Psi_{L}^{(n)}}
\end{gathered}
$$

where we have defined $M_{\Psi}=c k$ The corresponding KK decomposition is [51]

$$
\Psi_{L R}(x, z)=\sum_{n} \Psi_{L R}^{(n)}(x) f_{L R}^{(n)}(z)
$$

therefore $f_{L}^{(n)}(z)$ must satısfy

$$
\left[\partial_{z}-z^{-1}(2+c)\right]\left[\partial_{z}-z^{-1}(2-c)\right] f_{L}^{(n)}(z)=-m_{n}^{2} f_{L}^{(n)}
$$

Thıs differential equation has the solution

$$
f_{L}^{(n)}(z)=A_{n}^{f}(k z)^{5 / 2}\left[J_{c+1 / 2}\left(m_{n} z\right)+B_{n}^{f} Y_{c+1 / 2}\left(m_{n} z\right)\right]
$$

where the constants are defined by the BCs and the orthonormality condition [51],

$$
\int_{L_{0}}^{L_{1}} d z \frac{1}{(k z)^{4}} f^{(n)}(z) f^{(m)}(z)=\delta_{m n}
$$

which can be derived by the same procedure as that of the gauge bosons Similarly to the gauge boson case, we define the basis function $S_{c}(m, z)$ as

$$
S_{c}\left(m_{n}, z\right)=\frac{\pi m_{n}}{2 k}(k z)^{5 / 2}\left[J_{c+\frac{1}{2}}\left(m_{n} L_{0}\right) Y_{c+\frac{1}{2}}\left(m_{n} z\right)-J_{c+\frac{1}{2}}\left(m_{n} z\right) Y_{c+\frac{1}{2}}\left(m_{n} L_{0}\right)\right],
$$

Up to a normalization constant, this basis function is the profile of a left-handed fermion with a Dirıchlet boundary condition at the Planck brane, $S_{c}^{+}$The profile of the corresponding right-handed chiral partner, $S_{c}^{+}$, has a Neumann BC at the Planck brane and is defined by Eq (C 30) Similarly, the profile of a right-handed fermion 
with a Dirichlet $\mathrm{BC}$ at the Planck brane, $S_{c}^{-}$, is given by reversing the sign of $c$ in Eq. (3.30); its chiral partner, $\dot{S}_{c}^{-}$, is defined by Eq. (C.31). Therefore we have

$$
\begin{gathered}
S_{c}^{ \pm}\left(m_{n}, z\right)=S_{ \pm c}\left(m_{n}, z\right) \\
\dot{S}_{c}^{ \pm}\left(m_{n}, z\right)=\mp \frac{1}{m_{n}}\left[\partial_{z}-\frac{1}{z}(2 \mp c)\right] S_{ \pm c}\left(m_{n}, z\right) .
\end{gathered}
$$

The left-handed zero mode fermion profile can be found by solving Eq.

with $m_{0}=0$. Taking Neumann BCs where the brane kinetic terms have been ne-

glected [51], the solution has the form $f^{(n)}(z)=A^{f} z^{2-c}$. Using the orthonormality condition we find that,

$$
f_{L}^{(0)}(z)=\sqrt{\frac{k(1-2 c)}{\left(k L_{1}\right)^{1-2 c}-1}}(k z)^{2-c} .
$$

A right-handed zero mode fermion profile can be obtained by substituting $c \rightarrow-c$ in Eq. (C.39).

\section{C.2.3 Higgs Profile}

Let the Higgs have the form $H_{5}(x, z)=H(x) f_{H}(z)$, and consider the square of the second gauge fixing term in Eq. (3.9). Substituting in our form for $H_{5}(x, z)$, it can be seen that when this term is integrated over $z$, the effective 4-dimensional term will be a mass term.

$$
\left[\epsilon z \partial_{z}\left(\frac{1}{z} H_{5}(x, z)\right)\right]^{2}=\left[\epsilon z \partial_{z}\left(\frac{1}{z} f_{H}(z)\right)\right]^{2} H(x)^{2}
$$

For the zero-mode the mass must be zero before EWSB. Therefore the factor preceding $H(x)^{2}$ must be equal to zero, yielding the relation

$$
\partial_{z}\left(\frac{f_{H}(z)}{z}\right)=0
$$


Solving this differential equation, we find that the profile of $A_{5}^{a(0)}$ is $f_{H}(z)=c z$ where $c$ is a constant Using the orthonormalization condition of $\mathrm{Eq}$ (C 18) we find that $[3,46]$

$$
f_{H}(z)=z \sqrt{\frac{2 k}{L_{1}^{2}-L_{0}^{2}}}
$$

\section{C.3 Profiles with particle mixing}

In this section we provide a detaled overview of the fully mixed particle profiles after EWSB Explicit calculations and summaries can also be found in references $[4,50,52,64]$, however, it should be noted that these sources define the rotation angle $\theta_{H}$ in a different way Furthermors, therr profile coefficient solutions often take a different form from ours, duc to differences in the solution method However, the results are equivalent

In the interest of notational clarity, we will omit the superscript $n$ denoting the KK mode on all profiles $f^{(n)}$ and coefficients $C_{G}^{(n)}$ throughout this section, the order dependence will be expressed solely through the subscript on the mass parameters $m_{n}$

\section{C.3.1 Gauge Bosons}

As indicated in the text, the fully mixed particle profiles with Higgs vev $v, f\left(m_{n}, z, v\right)$, can be obtained from the pre-EWSB basis profiles through the gauge transformation given in Eq $(341)$ The base profiles $f\left(m_{n}, z, 0\right)$ of the bosons are defined by 
$\mathrm{Eq}(\mathrm{C} 21)$ as

$$
\begin{aligned}
f_{a_{L}}\left(m_{n}, z, 0\right)=C_{a_{L}} C_{A}\left(m_{n}, z\right), & f_{a}\left(m_{n}, z, 0\right)=C_{a} S_{A}\left(m_{n}, z\right), \\
f_{1_{R}}\left(m_{n}, z, 0\right)=C_{1_{R}} S_{A}\left(m_{n}, z\right), & f_{2_{R}}\left(m_{n}, z, 0\right)=C_{2_{R}} S_{A}\left(m_{n}, z\right), \\
f_{X}\left(m_{n}, z, 0\right)=C_{X} S_{A}\left(m_{n} z\right), & f_{B}\left(m_{n}, z, 0\right)=C_{B} C_{A}\left(m_{n}, z\right),
\end{aligned}
$$

where $C_{A}\left(m_{n}, z\right)$ and $S_{A}\left(m_{n}, z\right)$ are the basis functions defined in Eqs (C 19-C 20), and the $C_{G}$ are normalization coefficients [4] Note that it is $f_{X}\left(m_{n}, z, 0\right)$ and $f_{B}\left(m_{n}, z, 0\right)$ that are defined in terms of the basis functions, rather than $f_{3_{R}}\left(m_{n}, z, 0\right)$ and $f_{U}\left(m_{n}, z, 0\right)$ This is because $C_{A}\left(m_{n} z\right)$ and $S_{A}\left(m_{n}, z\right)$ are solutions with Neumann and Dirıchlet BCs at the Planck brane respectively, and it is upon $X$ and $B$ that the boundary conditions are defined We define $f_{3_{R}}\left(m_{n}, z, 0\right)$ and $f_{U}\left(m_{n} z, 0\right) \mathrm{m}$ terms of the $X$ and $B$ profiles in the work below

In the 5-representation of the $\mathrm{SO}(5)$ generators (please see Appendix B), the transformation matrix $\Omega(z, v)$ of $\mathrm{Eq}$ (3 42) can be rewritten as

$$
\Omega(z, v)=1-\imath \sqrt{2} T^{4} \sin \theta_{G}+\sum_{\imath=4}^{5} \delta_{\imath \iota}\left(\cos \theta_{G}-1\right)
$$

where $\theta_{G}=\theta_{G}(z, v)$ is defined in Eq (343) In this representation the mixed profiles are found to be,

$$
\begin{gathered}
f_{1_{L R}}(v)=\frac{1}{2}\left(1 \pm \cos \theta_{G}\right) C_{1_{L}} C_{A}+\frac{1}{2}\left(1 \mp \cos \theta_{G}\right) C_{1_{R}} S_{A} \pm \frac{\sqrt{2}}{2} \sin \theta_{G} C_{1} S_{A}, \\
f_{2_{L R}(v)=} \frac{1}{2}\left(1 \pm \cos \theta_{G}\right) C_{2_{L}} C_{A}+\frac{1}{2}\left(1 \mp \cos \theta_{G}\right) C_{R_{R}} S_{A} \pm \frac{\sqrt{2}}{2} \sin \theta_{G} C_{2} S_{A} \\
f_{3_{L R}}(v)=\frac{1}{2}\left(1 \pm \cos \theta_{G}\right) C_{3_{L}} C_{A} \pm \frac{\sqrt{2}}{2} \sin \theta_{G} C_{3} S_{A} \\
+\frac{1}{2}\left(1 \mp \cos \theta_{G}\right)\left[\cos \theta_{H} C_{B} C_{A}-\sin \theta_{H} C_{X} S_{A}\right]
\end{gathered}
$$




$$
\begin{gathered}
f_{\hat{1}}(v)=\cos \theta_{G} C_{\hat{1}} S_{A}+\frac{1}{\sqrt{2}} \sin \theta_{G}\left(C_{1_{R}} S_{A}-C_{1_{L}} C_{A}\right), \\
f_{\hat{2}}(v)=\cos \theta_{G} C_{\hat{2}} S_{A}+\frac{1}{\sqrt{2}} \sin \theta_{G}\left(C_{2_{R}} S_{A}-C_{2_{L}} C_{A}\right), \\
f_{\hat{3}}(v)=\cos \theta_{G} C_{\hat{3}} S_{A}+\frac{1}{\sqrt{2}} \sin \theta_{G}\left(\cos \theta_{H} C_{B} C_{A}-\sin \theta_{H} C_{X} S_{A}-C_{3_{L}} C_{A}\right), \\
f_{\hat{4}}(v)=C_{\hat{4}} S_{A}, \\
f_{U}(v)=\sin \theta_{H} C_{B} C_{A}+\cos \theta_{H} C_{X} S_{A} .
\end{gathered}
$$

where we have suppressed the $z$ and $m_{n}$ dependence; $f_{G}(v)=f_{G}\left(m_{n}, z, v\right), C_{A}=$ $C_{A}\left(m_{n}, z\right)$, and $S_{A}=S_{A}\left(m_{n}, z\right)$ [64]. Recall that $\cos \theta_{H}=\tan \theta_{w}$.

As discussed in section 4.3 , the $\mathrm{TeV}$ boundary conditions provide a system of equations by which the coefficients $C_{G}$ and mass eigenvalues may be determined. The boson masses are determined by setting the determinant of this system to zero, a requirement for the system to be solvable. The system of equations can be broken into four subsystems.

\section{The $W$ boson sector}

The first two subsystems are mathematically idontical, the only difference being that one involves the $W^{1_{L}}, W^{1_{R}}$ and $A^{\hat{1}}$ bosons while the other involves the $W^{2_{L}}, W^{2_{R}}$ and $A^{\hat{2}}$ bosons. We will represent both of these systems by $W^{\imath_{L}}, W^{i_{R}}$ and $A^{i}$ - where $i=1,2$ - with the corresponding system of TeV-brane boundary conditions

$$
\begin{gathered}
\left.\partial_{z} f_{i_{L}}^{(n)}\left(m_{n}, z ; v\right)\right|_{z=L_{1}}=0 \\
\left.\partial_{z} f_{i_{R}}^{(n)}\left(m_{n}, z ; v\right)\right|_{z=L_{1}}=0 \\
\left.f_{i}^{(n)}\left(m_{n}, z ; v\right)\right|_{z=L_{1}}=0
\end{gathered}
$$


which we use to solve for $C_{\imath_{L}}, C_{\imath_{R}}$ and $C_{\hat{\imath}}[64]$. Setting the determinant of this system to zero, we obtain the mass relations

$$
\begin{gathered}
S_{A}^{\prime}\left(m_{n}, L_{1}\right)=0 \\
2 C_{A}^{\prime}\left(m_{n}, L_{1}\right) S_{A}\left(m_{n}, L_{1}\right)+m_{n} k L_{1} \sin \theta_{G}^{2}\left(L_{1}, v\right)=0
\end{gathered}
$$

where a prime represents the derivative with sespect to the fifth coordinate $z$ and we have used the Wronskian relation $[4,62],{ }^{4}$

$$
S_{A}^{\prime}\left(m_{n}, z\right) C_{A}\left(m_{n}, z\right)-S_{A}\left(m_{n}, z\right) C_{A}^{\prime}\left(m_{n}, z\right)=m_{n} k z
$$

Eq. (C.58) corresponds to the $W^{\imath_{R}}$ state masses, which are the same as the original masses before mixing (Eq. (C.23)). Eq. (C.59) corresponds to the combined $W^{\imath_{L}}$ and $W^{\hat{\imath}}$ masses; note that as $v \rightarrow 0$ the left-hand side becomes the product of the left-hand sides of their original mass equations, $C_{A}^{\prime}\left(m_{n}, L_{1}\right) S_{A}\left(m_{n}, L_{1}\right)$ (Eq. (C.22) and Eq. (C.24)).

Because there are two conditions that allow the determinant to be zero, there are two possible solutions for the coefficient set. The first, corresponding to the $W^{\imath_{L}}$ mass condition, Eq. (C.59), is

$$
\begin{aligned}
& C_{\imath_{L}}^{W_{L}}=\sqrt{2} C_{\hat{\imath}} \frac{S_{A}\left(m_{n}, L_{1}\right)\left(1+\cos \theta_{G}^{2}\left(L_{1}, v\right)\right)}{C_{A}\left(m_{n}, L_{1}\right) \sin 2 \theta_{G}\left(L_{1}, v\right)} \\
& C_{\imath_{R}}^{W_{L}}=\frac{\sqrt{2}}{2} C_{\hat{\imath}} \tan \theta_{G}\left(L_{1}, v\right), \quad C_{\hat{\imath}}^{W_{L}}=C_{\hat{\imath}} .
\end{aligned}
$$

where the remaining coefficient $C_{\hat{\imath}}$ will be determined by the normalization condition, and the superscript $W_{L}$ identifies the coefficient set.

When these coefficients are substituted into the profiles $f_{\imath_{L}}(m, z ; v)$, $f_{\imath_{R}}(m, z ; v)$ and $f_{\hat{\imath}}(m, z ; v)$, all of these profiles are non-zero. This indicates that the

\footnotetext{
${ }^{4}$ Also known as Abel's identity.
} 
full $W^{\imath_{L}}$ mass eigenstate profile is a superposition of these mixed profiles, weighted by their associated generators,

$$
F_{\imath_{L}}(m, z ; v) T^{F_{2 L}}=f_{\imath_{L}}^{W_{L}}(m, z ; v) T^{\imath_{L}}+\int_{\imath_{R}}^{W_{L}}(m, z ; v) T^{\imath_{R}}+f_{\hat{\imath}}^{W_{L}}(m, z ; v) T^{\hat{\imath}} .
$$

where the superscript $W_{L}$ on the profiles indicates that coefficients $C_{\imath_{L}}, C_{\imath_{L}}$, and $C_{\hat{\imath}}$ have been replaced by those in Eq. (C.61). Here $T^{F_{2 L}}$ is meant to denote the generator that would be associated with the profile $F_{\imath L}(m, z ; v)$; however, in practice the two are never separated. Depending upon the mass solution of Eq. (C.59) with which it is evaluated, Eq. (C.62) may represent a mass eigenstate that corresponds to either the pre-EWSB $A_{\mu}^{\imath_{L}}$, or the pre-EWSB $A_{\mu}^{\hat{z}}$.

The normalization condition for this profile is

$$
\int_{L_{0}}^{L_{1}} \frac{d z}{k z}\left(f_{\imath_{L}}^{\alpha}(m, z ; v)^{2}+f_{\imath_{R}}^{\alpha}(m, z ; v)^{2}+f_{i}^{\alpha}(m, z ; v)^{2}\right)=1
$$

The second solution set, corresponding to Eq. (C.58), is given by

$$
C_{\imath_{L}}^{W_{R}}=0, \quad C_{\imath_{R}}^{W_{R}}=-\sqrt{2} C_{\hat{\imath}} \tan \theta_{G}\left(L_{1}, v\right), \quad C_{\hat{\imath}}^{W_{R}}=C_{\hat{i}}
$$

The corresponding full profile $F_{2 L}(m, z ; v) T^{F_{2 R}}$ and its normalization condition are given by Eq. (C.62) and (C.63) with $W_{L} \rightarrow W_{R}$. We emphasize that the difference between the profiles of the three types of boson arises from the different coefficient sets obtained by solving Eqs. (C.55-C.57) and the corresponding mass eigenvalues. 


\section{The $\gamma$ and $Z$ sector}

The third subsystem involves the $Z, X, V$ and $A^{3}$ bosons where again we denote the photon by $V_{\mu} \quad$ with the corresponding system of boundary conditions

$$
\begin{array}{cc} 
& \left.\partial_{z} f_{3_{L}}^{(n)}\left(m_{n}, z, v\right)\right|_{z=L_{1}}=0, \\
\left.f_{3}^{(n)}\left(m_{n}, z, v\right)\right|_{z=L_{1}}=0 & \text { (C 65 66) } \\
\left.\partial_{z} f_{B}^{(n)}\left(m_{n}, z, v\right)\right|_{z=L_{1}}=\left.\partial_{z}\left[\cos \theta_{w} f_{3_{R}}^{(n)}\left(m_{n}, z, v\right)+\sin \theta_{w} f_{U}^{(n)}\left(m_{n}, z, v\right)\right]\right|_{z=L_{1}}=0 \\
\left.\partial_{z} f_{X}^{(n)}\left(m_{n}, z, v\right)\right|_{z=L_{1}}=\left.\partial_{z}\left[\cos \theta_{w} f_{U}^{(n)}\left(m_{n} z, v\right)-\sin \theta_{w} f_{3_{R}}^{(n)}\left(m_{n}, z, v\right)\right]\right|_{z=L_{1}}=0
\end{array}
$$

which we use to solve for $C_{3_{L}}, C_{X}, C_{B}$ and $C_{3}$ [64] Setting the determinant of this system to zero, we obtain the mass relations

$$
\begin{gathered}
S_{A}^{\prime}\left(m_{n}, L_{1}\right)=0, \\
C_{A}^{\prime}\left(m_{n} L_{1}\right)=0, \\
2 C_{A}^{\prime}\left(m_{n}, L_{1}\right) S_{A}\left(m_{n}, L_{1}\right)+m_{n} k L_{1} \sec ^{2} \theta_{w} \sin \theta_{G}^{2}\left(L_{1}, v\right)=0
\end{gathered}
$$

The first two equations correspond to the masses of the $X$ boson and photon state respectively, both are the same as the original masses before mixing (Eq (C 23) and Eq (C 22)) In particular, the zero-mode photon remains massless The third corresponds to the combined $Z$ and $A^{3}$ masses, again, as $v \rightarrow 0$ the left-hand side of this equation becomes the product of the left-hand sides of the original $Z$ and $A^{3}$ mass equations, $C_{A}^{\prime}\left(m_{n}, L_{1}\right) S_{A}\left(m_{n}, L_{1}\right)$

In this case there are three possible solutions for the coefficient set The first, corresponding to the $X$ boson mass condition, Eq (C 69), is

$$
C_{3_{L}}^{X}=C_{B}^{X}=0, \quad C_{X}^{1}=\sqrt{2} C_{3} \tan \theta_{G}\left(L_{1}, v\right) \sin \theta_{H}, \quad C_{3}^{X}=C_{3}
$$


where the superscript is meant to identify the mass condition associated with the coefficient solution set.

The second solution set, corresponding to Eq. (C.71), is given by

$$
\begin{gathered}
C_{B}^{Z}=\cos \theta_{H} C_{3_{L}}^{Z}=C_{X} \frac{S_{A}\left(m_{n}, L_{1}\right)}{C_{A}\left(m_{n}, L_{1}\right)} \frac{\left[1-\cos ^{2} \theta_{H}+\cos ^{2} \theta_{G}\left(L_{1}, v\right)\left(1+\cos ^{2} \theta_{H}\right)\right]}{\sin \theta_{H}\left(-1+\cos ^{2} \theta_{G}\left(L_{1}, v\right)\right)\left(1+\cos ^{2} \theta_{H}\right)}, \\
C_{X}^{Z}=C_{X}, \quad C_{\hat{3}}^{Z}=\frac{\sqrt{2} C_{X}}{\tan \theta_{G}\left(L_{1}, v\right) \sin \theta_{H}} .
\end{gathered}
$$

and the third, corresponding to the photon mass condition, Eq. (C.70), is given by

$$
C_{3_{L}}^{V}=C_{B} \cos \theta_{H}, \quad C_{X}^{V}=C_{\hat{3}}^{V}=0, \quad C_{B}^{V}=C_{B}
$$

When the coefficients corresponding to the $X$ and $Z$ mass conditions Eq. (C.72) and Eq. (C.73) respectively - are substituted into the profiles $f_{3_{L}}(m, z ; v)$, $f_{X}(m, z ; v), f_{B}(m, z ; v)$ and $f_{\hat{3}}(m, z ; v)$, all of them are non-zero. However, while the profile of the $Z$ boson

$$
f_{Z}(m, z ; v)=\cos \theta_{w} f_{3_{L}}(m, z ; v)-\sin \theta_{w} f_{B}(m, z ; v)
$$

is also non-zero, it turns out that the profile of the photon

$$
f_{V}(m, z ; v)=\cos \theta_{w} f_{B}(m, z ; v)+\sin \theta_{w} f_{3_{L}}(m, z ; v)
$$

disappears. The reverse is also truc; when the cocficients corresponding to the photon mass condition, Eq. (C.70), are substituted into the profiles, only the photon profile is non-zero. As a result, the final profiles are the following,

$$
\begin{gathered}
F_{X}(m, z ; v) T^{F_{X}}=f_{Z}^{X}(m, z ; v) T^{Z}+f_{X}^{X}(m, z ; v) T^{X}+f_{\hat{3}}^{X}(m, z ; v) T^{\hat{3}} \\
F_{Z}(m, z ; v) T^{F_{Z}}=f_{Z}^{Z}(m, z ; v) T^{Z}+f_{X}^{Z}(m, z ; v) T^{X}+f_{\hat{3}}^{Z}(m, z ; v) T^{\hat{3}} \\
F_{V}(m, z ; v)=\cos \theta_{w} f_{B}^{V}(m, z ; v)+\sin \theta_{w} f_{3_{L}}^{V}(m, z ; v)
\end{gathered}
$$


where, as in the previous section, the superscipt indicates which set of coefficients has been applied The normalization condition for each profile is analogous to Eq (C 63) Notice that the photon profile is unaffected by the mixing

The $A_{\mu}^{4}$ sector.

The $A_{\mu}^{4}$ boson does not mix with any other bosons, as such, its final profile is simply

$$
F_{4}(m, z, v)=f_{4}(m, z, v)=f_{4}(m, z, 0)=C_{4} S_{A}(m, z)
$$

\section{C.3.2 Fermions}

The procedure for the fermions is very sumilar to that of the gauge bosons, after performing the gauge transformation, we solve the coefficient matrices and obtain mass conditions from the matrix determinants

The gauge transformation is slightly different for fermions First, we write the fermion multiplets - in this case the lepton multiplets - in form

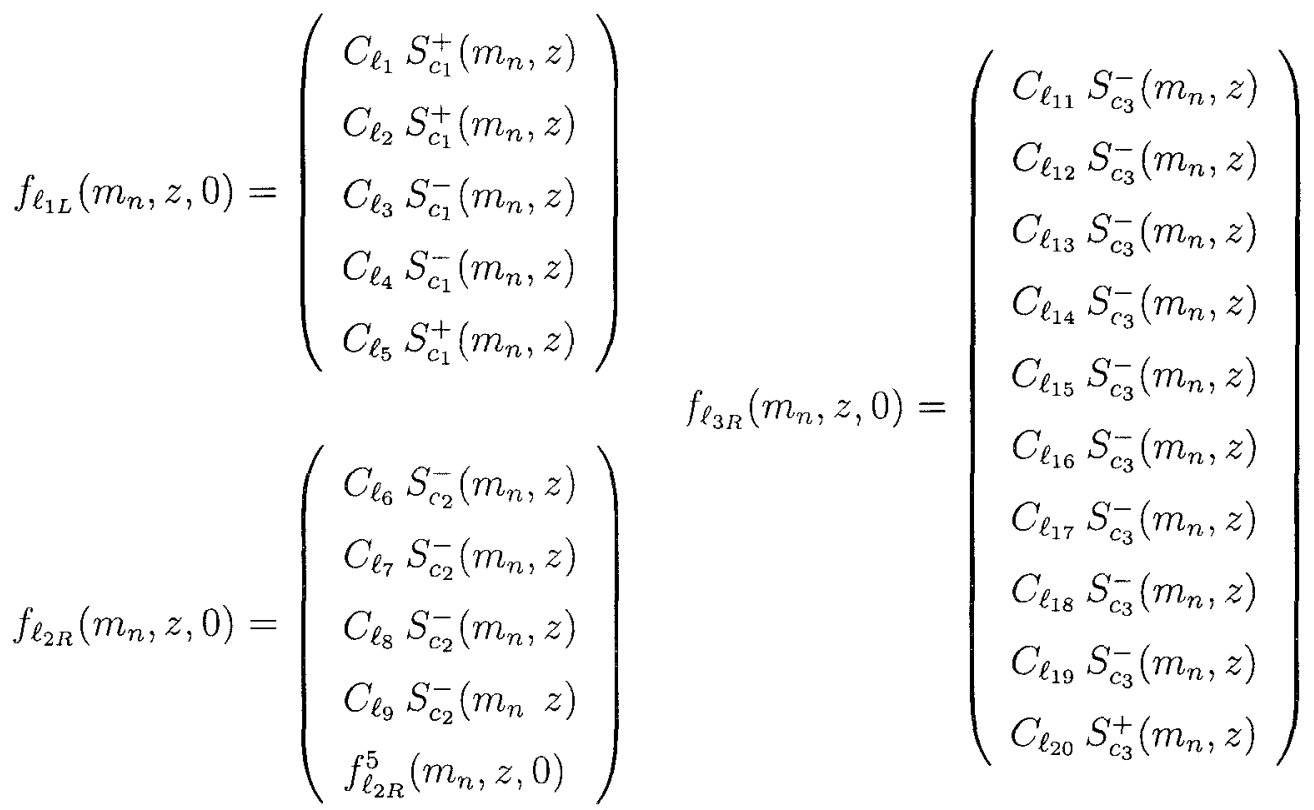


where $S_{c}^{ \pm}$and $S_{c}^{ \pm}$are the fermon basis functions defined by Eqs $(3$ 30-3 31) [4, 52, 64], and

$$
\begin{gathered}
f_{\ell_{2 L}}^{5}\left(m_{n}, z, 0\right)=C_{\ell_{10}}\left(S_{c_{2}}^{+}-M_{U V} S_{c_{2}}^{-}\right) \\
f_{\ell_{2 R}}^{5}\left(m_{n}, z, 0\right)=C_{\ell_{10}}\left(-M_{U V} S_{c_{2}}^{-}+S_{c_{2}}^{+}\right)
\end{gathered}
$$

The vectors of opposite chirality can be read from these by replacing $S_{c}^{ \pm} \leftrightarrow S_{c}^{ \pm}$and $f_{\ell_{2 R}}^{5} \rightarrow f_{\ell_{2 L}}^{5}$ The quark profile vectors $-f_{\xi_{1 L}}, f_{\xi_{2 R}}$ and $f_{\xi_{3 R}}$ have an identical vector form, except that in their case $M_{U V}=0$

However, this form does not belong to the same basis as our $\mathrm{SO}(5)$ generators in Appendix B Therefore we must tiansform these vectors into our generator basis before the gauge transformation can be applied As such, our gauge transformation takes the following form in the fermion case [4, 50],

$$
\begin{aligned}
& f_{\ell_{1 L}}\left(m_{n} z, v\right)=A \Omega(z, v) A^{-1} f_{\ell_{1 L}}\left(m_{n} z, 0\right), \\
& f_{\ell_{2 R}}\left(m_{n}, z, v\right)=A \Omega(z v) A^{-1} f_{\ell_{2 R}}\left(m_{n}, z, 0\right), \\
& f_{\ell_{3 R}}\left(m_{n}, z, v\right)=B \Omega(z, v) B^{-1} f_{\ell_{3 R}}\left(m_{n}, z, 0\right)
\end{aligned}
$$

where the $T^{4}$ gencrator in $\Omega(z, v)$ is in 5 -represcntation for the first two cquations and 10-representation for the third The change of-basis matrices $A$ and $B$ are given by $[4,50]$

$$
A=\frac{1}{\sqrt{2}}\left(\begin{array}{ccccc}
-\imath & -1 & 0 & 0 & 0 \\
0 & 0 & -\imath & 1 & 0 \\
0 & 0 & \imath & 1 & 0 \\
-\imath & 1 & 0 & 0 & 0 \\
0 & 0 & 0 & 0 & \sqrt{2}
\end{array}\right)
$$


and

$$
B=\frac{1}{\sqrt{2}}\left(\begin{array}{llllllllll}
\imath & 1 & 0 & 0 & 0 & 0 & 0 & 0 & 0 & 0 \\
0 & 0 & -\imath & 1 & 0 & 0 & 0 & 0 & 0 & 0 \\
0 & 0 & -\imath & -1 & 0 & 0 & 0 & 0 & 0 & 0 \\
-\imath & 1 & 0 & 0 & 0 & 0 & 0 & 0 & 0 & 0 \\
0 & 0 & 0 & 0 & -1 & \imath & 0 & 0 & 0 & 0 \\
0 & 0 & 0 & 0 & 0 & 0 & \sqrt{2} & 0 & 0 & 0 \\
0 & 0 & 0 & 0 & 1 & \imath & 0 & 0 & 0 & 0 \\
0 & 0 & 0 & 0 & 0 & 0 & 0 & -1 & \imath & 0 \\
0 & 0 & 0 & 0 & 0 & 0 & 0 & 0 & 0 & \sqrt{2} \\
0 & 0 & 0 & 0 & 0 & 0 & 0 & 1 & \imath & 0
\end{array}\right)
$$

Performing this transformation, we obtain the following mixed profiles

$$
f_{\ell_{1 L}}(v)=\left(\begin{array}{c}
C_{\ell_{1}} S_{c_{1}}^{+} \\
\cos ^{2}\left(\frac{\theta_{G}}{2}\right) C_{\ell_{2}} S_{c_{1}}^{+}-\sin ^{2}\left(\frac{\theta_{G}}{2}\right) C_{\ell_{3}} \dot{S}_{c_{1}}^{-}-\frac{\sqrt{2}}{2} \sin \theta_{G} C_{\ell_{5}} S_{c_{1}}^{+} \\
-\sin ^{2}\left(\frac{\theta_{G}}{2}\right) C_{\ell_{2}} S_{c_{1}}^{+}+\cos ^{2}\left(\frac{\theta_{G}}{2}\right) C_{\ell_{3}} \dot{S}_{c_{1}}^{-}-\frac{\sqrt{2}}{2} \sin \theta_{G} C_{\ell_{5}} S_{c_{1}}^{+} \\
C_{\ell_{4}} \dot{S}_{c_{1}}^{-} \\
f_{\ell_{2 R}}(v)=\left(\begin{array}{c}
\frac{\sqrt{2}}{2} \sin \theta_{G} C_{\ell_{2}} S_{c_{1}}^{+}+\frac{\sqrt{2}}{2} \sin \theta_{G} C_{\ell_{3}} \dot{S}_{c_{1}}^{-}+\cos \theta_{G} C_{\ell_{5}} S_{c_{1}}^{+} \\
-\sin ^{2}\left(\frac{\theta_{G}}{2}\right) C_{\ell_{7}} S_{c_{2}}^{-}+\cos ^{2}\left(\frac{\theta_{G}}{2}\right) C_{\ell_{8}} S_{c_{2}}^{-}-\frac{\sqrt{2}}{2} \sin \theta_{G} f_{\ell_{2 R}}^{5}(0) \\
C_{\ell_{9}} S_{c_{2}}^{-} \\
\frac{\cos ^{2}\left(\frac{\theta_{G}}{2}\right) C_{\ell_{7}} S_{c_{2}}^{-}-\sin ^{2}\left(\frac{\theta_{G}}{2}\right) C_{\ell_{8}} S_{c_{2}}^{-}-\frac{\sqrt{2}}{2} \sin \theta_{G} f_{\ell_{2 R}}^{5}(0)}{\frac{\sqrt{2}}{2} \sin \theta_{G} C_{\ell_{7}} S_{c_{2}}^{-}+\frac{\sqrt{2}}{2} \sin \theta_{G} C_{\ell_{8}} S_{c_{2}}^{-}+\cos \theta_{G} f_{\ell_{2 R}}^{5}(0)}
\end{array}\right)
\end{array}\right.
$$




$$
f_{\ell_{3 R}}(v)=\left(\begin{array}{c}
\cos \theta_{G} C_{\ell_{11}} S_{c_{3}}^{-}+\frac{i \sqrt{2}}{2} \sin \theta_{G}\left(C_{\ell_{18}}-C_{\ell_{15}}\right) S_{c_{3}}^{-} \\
\cos ^{2}\left(\frac{\theta_{G}}{2}\right) C_{\ell_{12}} S_{c_{3}}^{-}-\sin ^{2}\left(\frac{\theta_{G}}{2}\right) C_{\ell_{13}} S_{c_{3}}^{-}+\frac{\imath}{2} \sin \theta_{G}\left(C_{\ell_{19}}-C_{\ell_{16}}\right) S_{c_{3}}^{-} \\
\cos ^{2}\left(\frac{\theta_{G}}{2}\right) C_{\ell_{13}} S_{c_{3}}^{-}-\sin ^{2}\left(\frac{\theta_{G}}{2}\right) C_{\ell_{12}} S_{c_{3}}^{-}+\frac{2}{2} \sin \theta_{G}\left(C_{\ell_{19}}-C_{\ell_{16}}\right) S_{c_{3}}^{-} \\
\cos \theta_{G} C_{\ell_{14}} S_{c_{3}}^{-}-\frac{\imath \sqrt{2}}{2} \sin \theta_{G} C_{\ell_{17}} S_{c_{3}}^{-}+\frac{\imath \sqrt{2}}{2} \sin \theta_{G} C_{\ell_{20}} \dot{S}_{c_{3}}^{+} \\
-\frac{2 \sqrt{2}}{2} \sin \theta_{G} C_{\ell_{11}} S_{c_{3}}^{-}+\cos ^{2}\left(\frac{\theta_{G}}{2}\right) C_{\ell_{15}} S_{c_{3}}^{-}-\sin ^{2}\left(\frac{\theta_{G}}{2}\right) C_{\ell_{18}} S_{c_{3}}^{-} \\
\cos ^{2}\left(\frac{\theta_{G}}{2}\right) C_{\ell_{16}} S_{c_{3}}^{-}-\sin ^{2}\left(\frac{\theta_{G}}{2}\right) C_{\ell_{19}} S_{c_{3}}^{-}-\frac{1}{2} \sin \theta_{G}\left(C_{\ell_{12}}+C_{\ell_{13}}\right) S_{c_{3}}^{-} \\
-\frac{i \sqrt{2}}{2} \sin \theta_{G} C_{\ell_{14}} S_{c_{3}}^{-}+\cos ^{2}\left(\frac{\theta_{G}}{2}\right) C_{\ell_{17}} S_{c_{3}}^{-}-\sin ^{2}\left(\frac{\theta_{G}}{2}\right) C_{\ell_{20}} \dot{S}_{c_{3}}^{+} \\
\frac{2 \sqrt{2}}{2} \sin \theta_{G} C_{\ell_{11}} S_{c_{3}}^{-}+\cos ^{2}\left(\frac{\theta_{G}}{2}\right) C_{\ell_{18}} S_{c_{3}}^{-}-\sin ^{2}\left(\frac{\theta_{G}}{2}\right) C_{\ell_{15}} S_{c_{3}}^{-} \\
\frac{2}{2} \sin \theta_{G}\left(C_{\ell_{1} 2}+C_{\ell_{13}}\right) S_{c_{3}}^{-}-\sin ^{2}\left(\frac{\theta_{G}}{2}\right) C_{\ell_{16}} S_{c_{3}}^{-}+\cos ^{2}\left(\frac{\theta_{G}}{2}\right) C_{\ell_{19}} S_{c_{3}}^{-} \\
\frac{i \sqrt{2}}{2} \sin \theta_{G} C_{\ell_{14}} S_{c_{3}}^{-}-\sin ^{2}\left(\frac{\theta_{G}}{2}\right) C_{\ell_{17}} S_{c_{3}}^{-}+\cos ^{2}\left(\frac{\theta_{G}}{2}\right) C_{\ell_{20}} \dot{S}_{c_{3}}^{+}
\end{array}\right)
$$

In terms of the vectorial form, the fermion boundary conditions of Eq. (3.37-3.39) can be written as

$$
\begin{array}{ccc}
f_{\ell_{1 L}}^{1,, 4}+M_{2} f_{\ell_{3 R}}^{1,, 4}=0 & f_{\ell_{1 L}}^{5}+M_{2} f_{\ell_{2 R}}^{5}=0 & f_{\ell_{2 L}}^{1, ., 4}=0 \\
f_{\ell_{3 L}}^{1,, 4}-M_{2} f_{\ell_{1 L}, 4}^{1,4}=0 & f_{\ell_{2 L}}^{5}-M_{1} f_{\ell_{1 L}}^{5}-M_{I R} f_{\ell_{2 R}}^{5}=0 & f_{\ell_{3 L}}^{5,, 10}=0
\end{array}
$$

where the superscript denotes the vector component $[4,50,52]$. The quark profile boundary conditions are exactly the same, except that $M_{I R}=0$.

As in the boson case, this system of equations can be divided into three independent subsystems, one for each fermion EM charge. In the lepton case we have charges 1,0 and -1 , while in the quark case we have $5 / 3,2 / 3$ and $-1 / 3$.

In the following three subsections we will be dealing exclusively with the 
fermion mass conditions and coefficient sets, which are all evaluated at the $\mathrm{TeV}$ brane As such, we will omit any notational indication of functional dependence on the vev $v$, mass $m_{n}$, or the coordmate $z=L_{1}$ in $S_{c}^{ \pm}, S_{c}^{ \pm}$and $\theta_{G}$

Exotic fermions $(Q=5 / 3,1)$

The first sector is entircly composed of exotic fermions with charge $5 / 3$ that do not appear in the Standard Model There are five equations corresponding to these termions, four can be solved for corfficients $C_{\ell_{1}}, C_{\ell_{11}}, C_{\ell_{15}}$ and $C_{\ell_{18}}$, while the fitth contains only $C_{\ell_{6}}$ and is independent of the others

The determmant of the corresponding matrix yeelds the following mass condltions,

$$
\begin{gathered}
S_{c_{3}}{ }^{2}=0 \\
S_{c_{1}}^{+} S_{c_{3}}^{-}+M_{2}^{2} S_{c_{1}}^{+} S_{c_{3}}^{-}=0
\end{gathered}
$$

where all functions are evaluated at $z=L_{1}$

The first mass condition corresponds to the following coefficients,

$$
\begin{gathered}
C_{\ell_{1}}^{\alpha \beta}=0, \quad C_{\ell_{11}}^{\alpha \beta}=\frac{\imath \sqrt{2}}{2} \tan \theta_{G}\left(C_{\ell_{15}}-C_{\ell_{18}}\right), \\
C_{\ell_{15}}^{\alpha \beta}=C_{\ell_{15}}, \quad C_{\ell_{18}}^{\alpha \beta}=C_{\ell_{18}},
\end{gathered}
$$

Because the condition appears twice the coefficient set is degenerate Any two perpendicular vectors in the $C_{\ell_{15}} C_{\ell_{18}}$ space will provide two independent coefficient sets We choose $\left(C_{\ell_{15}}, C_{\ell_{18}}\right)=(0,1)$ and $\left(C_{\ell_{15}}, C_{\ell_{18}}\right)=(1,0)$, we wlll label the former by superscript $\alpha$ and the second with superscript $\beta$ 
The second mass condition gives rise to a third coefficient set,

$$
C_{\ell_{1}}^{\gamma}=C_{\ell_{11}} \frac{\cos \theta_{G}}{M_{2}} \frac{\dot{S}_{c_{3}}^{-}}{S_{c_{1}}^{+}}, \quad C_{\ell_{11}}^{\gamma}=C_{\ell_{11}}, \quad C_{\ell_{15}}^{\gamma}=-C_{\ell_{18}}^{\gamma}=\frac{i \sqrt{2}}{2} \tan \theta_{G} C_{\ell_{11}} .
$$

which we denote by $\gamma$. Note that is a combined fermion state that corresponds to the mass eigenstates of two different fermions (refer to Table 3.6 for the specifics of the combination).

The independent equation has the trivial mass condition $\dot{S}_{\mathrm{c}_{2}}^{-}=0$, and the sole coefficient $C_{\ell_{6}}$ is the normalization coefficient. We will denote this solution set by $\delta$.

As such, in the end there are four exotic fermions per generation with profiles $F_{a_{L, R}}^{\frac{5}{3}}$ defined by their action on an arbitrary matrix $M$ as

$$
F_{a_{L, R}}^{\frac{5}{3} \dagger} M F_{a_{L, R}}^{\frac{5}{3}}=\sum_{i=1}^{3} f_{\ell_{\imath} L, 2 R}^{a \dagger} M f_{\ell_{\imath L, 2 R}}^{a}
$$

$a=\alpha, \beta, \gamma, \delta$, and subject to the normalization condition

$$
\int \frac{d z}{(k z)^{4}} \sum_{i=1}^{3} f_{\ell_{\imath L, 2 R}}^{a \dagger} f_{\ell_{2 L, 2 R}}^{a}=1
$$

where all profiles $f_{\ell}$ are evaluated at the same mass value. The notation $f_{\ell_{2}}^{a}$ indicates the vector $f_{\ell_{2}}$ with its coefficients evaluated according to coefficient set $a$; any coefficients undefined by the solution are set to zero. Note that in the case of the independent fermion ( $\delta$ solution set) Eq. (C.98) implies that

$$
F_{\delta_{L, R}}^{\frac{5}{3}}=C_{\ell_{6}} \dot{S}_{C_{2}}^{-}
$$

where $C_{\ell_{6}}$ is defined by the original fermion normalization condition, Eq. (C.35).

Down-type fermions $(Q=-1 / 3,-1)$

The second sector is composed of the appropriate Standard Model down-type fermion (depending on the fermion generation that we are considering) along with extra 
fermions that do not appear 11 the Standard Model As in the first sector, the one of the five equations involving these fermons - that involving $C_{\ell_{9}}$ is independent of the others The remaining four can be solved for coefhcients $C_{\ell_{4}}, C_{\ell_{14}}, C_{\ell_{17}}$ and $C_{\ell_{20}}$ The determinant of the corresponding $4 \times 4$ matrix yields the following mass conditions,

$$
\begin{gathered}
S_{c_{3}}^{-}=0 \\
2 S_{c_{3}}^{+}\left(M_{2}^{2} S_{c_{3}}^{-} S_{c_{1}}^{-}+S_{c_{1}}^{-} S_{c_{3}}^{-}\right)-(k z)^{4} M_{2}^{2} S_{c_{1}}^{-} \sin ^{2} \theta_{G}=0
\end{gathered}
$$

Here the second condition, Eq (C 102), corresponds to the mixed masses of the SM down-type fermion with one of the extra fermions, we will call the resulting fermion our down-type fermion The first conditıon, Eq (C 101), simply corresponds to another extra fermion

The first mass condition corresponds to the following coefficient set, which we denote by $\eta$,

$$
C_{\ell_{4}}^{\eta}=C_{\ell_{20}}^{\eta}=0, \quad C_{\ell_{14}}^{\eta}=\frac{\imath \sqrt{2}}{2} \tan \theta_{G} C_{\ell_{17}}, \quad C_{\ell_{17}}^{\eta}=C_{\ell_{17}}
$$

The second mass condition, corresponding to our down-type fermion $d$, gives rise to a second coefficient set,

$$
\begin{gathered}
C_{\ell_{4}}^{d}=\frac{\imath \sqrt{2}}{M_{2} \sin \theta_{G}} C_{\ell_{20}} \frac{S_{c_{3}}^{+}}{S_{c_{1}}^{-}}, \quad C_{\ell_{20}}^{d}=C_{\ell_{20}}, \\
C_{\ell_{14}}^{d}=-\frac{\imath \sqrt{2}}{M_{2} \tan \theta_{G}} C_{\ell_{17}}^{d}=\frac{\imath \sqrt{2}}{M_{2} \tan \theta_{G}} C_{\ell_{20}} \frac{S_{\mathrm{c}_{3}}^{+}}{S_{\mathrm{c}_{3}}^{-}},
\end{gathered}
$$

which we denote by $d$ This mass condition and coefficient set correspond to yet another combined profile, that includes the mass eigenstates of three fermions (see Table 36 )

The independent equation has the trivial mass condition $S_{c_{2}}^{-}=0$, and the sole coefficient $C_{\ell_{9}}$ is the normalization coefficient It corresponds to an extra fermion, we will denote it by $\epsilon$ 
In the end we have our down-type fermion with profile $F_{d_{L, R}}^{-\frac{1}{3}}$ and two extra fermions with profiles $F_{\epsilon_{L, R}}^{-\frac{1}{3}}$ and $F_{\eta_{L, R}}^{-\frac{1}{3}}$ The full profiles are defined by Eq. (C.98), where $a=d, \eta, \epsilon$.

\section{Up-type fermions $(Q=2 / 3,0)$}

The third sector is composed of the appropriate Standard Model up-type fermion along with extra fermions. In this case none of the equations are independent; all ten are solved together to define the remaining coefficients $C_{\ell_{2}}, C_{\ell_{3}}, C_{\ell_{5}}, C_{\ell_{7}}, C_{\ell_{8}}, C_{\ell_{10}}$ $C_{\ell_{12}}, C_{\ell_{13}}, C_{\ell_{16}}, C_{\ell_{19}}$. The resulting mass conditions are

$$
\begin{gathered}
\dot{S}_{c_{3}}^{-2}=0 \\
\dot{S}_{c_{2}}^{-}=0 \\
\dot{S}_{c_{1}}^{+} \dot{S}_{c_{3}}^{-}+M_{2}^{2} S_{c_{1}}^{+} S_{c_{3}}^{-}=0 \\
2 \dot{S}_{c_{2}}^{-}\left[S_{c_{1}}^{+} S_{c_{2}}^{-} M_{U V}\left(M_{2}^{2} M_{I R} \dot{S}_{c_{1}}^{-} S_{c_{3}}^{-}-M_{1}^{2} S_{c_{1}}^{-} \dot{S}_{c_{3}}^{-}\right)+\left(M_{2}^{2} \dot{S}_{c_{1}}^{-} S_{c_{3}}^{-}+S_{c_{1}}^{-} \dot{S}_{c_{3}}^{-}\right)\right. \\
\left.\times\left[M_{1}^{2} S_{c_{1}}^{+} \dot{S}_{c_{2}}^{+}+\dot{S}_{c_{1}}^{+}\left(S_{c_{2}}^{+}-M_{U V} \dot{S}_{c_{2}}^{-}-M_{I R} \dot{S}_{c_{2}}^{+}+M_{I R} M_{U V} S_{c_{2}}^{-}\right)\right]\right] \\
-\left[2\left(\dot{S}_{c_{1}}^{+} M_{I R}-S_{c_{1}}^{+}\right)\left(\dot{S}_{c_{1}}^{-} S_{c_{3}}^{-} M_{2}^{2}+\dot{S}_{c_{3}}^{-} S_{c_{1}}^{-}\right)+M_{2}^{2} \dot{S}_{c_{2}}^{-} S_{c_{3}}^{-}\left(M_{U V} \dot{S}_{c_{2}}^{-}-\dot{S}_{c_{2}}^{+}\right)\right. \\
\left.+\dot{S}_{c_{2}}^{-}\left(\dot{S}_{c_{2}}^{+}-M_{I R} S_{c_{2}}^{+}\right)\left(M_{2}^{2} M_{I R} S_{c_{3}}^{-}+M_{1}^{2} \dot{S}_{c_{3}}^{-}\right)\right](k z)^{4} \sin ^{2} \theta_{G} \\
-\left(M_{1}^{2} M_{I R} S_{c_{3}}^{-}+M_{1}^{2} \dot{S}_{c_{3}}^{-}\right)(k z)^{8} \sin ^{4} \theta_{G}=0
\end{gathered}
$$

Here the last condition corresponds to the mixed masses of the SM up-type fermion with one of the extra fermions; we will call the resulting fermion our up-type fermion. The other conditions simply correspond to other extra fermions.

The first mass condition yields two degencrate coefficient sets, which we denote 
by $\rho$ and $\alpha$,

$$
\begin{gathered}
C_{\ell_{2}}^{\rho, \sigma}=C_{\ell_{3}}^{\rho, \sigma}=C_{\ell_{5}}^{\rho, \sigma}=C_{\ell_{7}}^{\rho, \sigma}=C_{\ell_{8}}^{\rho, \sigma}=C_{\ell_{10}}^{\rho, \sigma}=0 \\
C_{\ell_{12}}^{\rho, \sigma}=C_{\ell_{13}}^{\rho, \sigma}=C_{\ell_{13}}, \quad C_{\ell_{16}}^{\rho, \sigma}=-\frac{2 i}{\tan \theta_{G}} C_{\ell_{13}}+C_{\ell_{19}}, \quad C_{\ell_{19}}^{\rho, \sigma}=C_{\ell_{19}} .
\end{gathered}
$$

Here again we choose $\left(C_{\ell_{13}}, C_{\ell_{19}}\right)=(0,1),(1,0)$ to obtain two independent solutions that we will denote by $\rho, \sigma$.

The second condition results in the coefficient set

$$
\begin{gathered}
C_{\ell_{2}}^{\tau}=C_{\ell_{3}}^{\tau}=C_{\ell_{5}}^{\tau}=C_{\ell_{10}}^{\tau}=C_{\ell_{12}}^{\tau}=C_{\ell_{13}}^{\tau}=C_{\ell_{16}}^{\tau}=C_{\ell_{19}}^{\tau}=0 \\
C_{\ell_{7}}^{\tau}=-C_{\ell_{8}}, \quad C_{\ell_{8}}^{\tau}=C_{\ell_{8}}
\end{gathered}
$$

which we denote by $\tau$, and the third results in

$$
\begin{gathered}
C_{\ell_{2}}^{\omega}=C_{\ell_{2}}, \quad C_{\ell_{3}}^{\omega}=C_{\ell_{7}}^{\omega}=C_{\ell_{8}}^{\omega}=C_{\ell_{10}}^{\omega}=C_{\ell_{13}}^{\omega}=0, \quad C_{\ell_{5}}^{\omega}=\frac{\sqrt{2}}{2} \tan \theta_{G} C_{\ell_{2}}, \\
C_{\ell_{16}}^{\omega}=-C_{\ell_{19}}^{\omega}=\frac{i}{2} \tan \theta_{G} C_{\ell_{12}}^{\omega}=\frac{i}{2} \tan \theta_{G} \frac{i}{2} M_{2} \tan \theta_{G} C_{\ell_{2}} \frac{S_{c_{1}}^{+}}{\dot{S}_{c_{3}}^{-}}
\end{gathered}
$$

which we will refer to by $\omega$. This $\omega$ coefficient set corresponds to a profile representing three fermion eigenstates merged into a combined tower (see Table 3.6).

Finally, the mass condition associated with the up-type fermion yields the coefficient set $u$,

$$
\begin{gathered}
C_{\ell_{2}}^{u}=\frac{1}{\sqrt{2}} \tan \theta_{G} C_{\ell_{5}}^{u}=C_{3} \frac{\dot{S}_{c_{3}}^{-} S_{c_{1}}^{-}+M_{2}^{2} S_{c_{1}}^{-} \dot{S}_{c_{1}}^{-}}{\dot{S}_{c_{1}}^{+} \dot{S}_{c_{3}}^{-}+M_{2}^{2} S_{c_{1}}^{+} S_{c_{3}}^{-}}, \quad C_{\ell_{3}}^{u}=C_{3}, \\
C_{\ell_{12}}^{u}=\frac{\sin ^{2} \theta_{g}}{1+\cos ^{2} \theta_{g}} C_{\ell_{13}}^{u}=i \tan \theta_{G} C_{\ell_{16}}^{u}=-i \tan \theta_{G} C_{\ell_{19}}^{u}=\frac{1}{2} \frac{M_{2} C_{3}(k z)^{4} \sin ^{2} \theta_{G}}{\dot{S}_{c_{1}}^{+} \dot{S}_{c_{3}}^{-}+M_{2}^{2} S_{c_{1}}^{+} S_{c_{3}}^{-}}, \\
C_{\ell_{7}}^{u}=C_{\ell_{8}}^{u}=\frac{1}{\sqrt{2}} \tan \theta_{G} \frac{S_{c_{2}}^{+}}{\dot{S}_{c_{2}}^{-}} C_{\ell_{10}}^{u}=-\frac{M_{1}}{M_{2}} \frac{\dot{S}_{c_{3}}^{-}}{\dot{S}_{c_{2}}^{-}} C_{\ell_{12}}^{u}+M_{1} \frac{S_{c_{1}}^{+}}{\dot{S}_{c_{2}}^{-}} C_{\ell_{2}}^{u}
\end{gathered}
$$

which represents five combined fermions. 
In the end we have our up-type fermion with plofile $F_{u_{L}}^{\frac{2}{3}}$ and four extra fermions with profiles $F_{\eta_{L} R}^{\frac{2}{3}}, F_{\rho_{L} R}^{2}, F_{\tau L R}^{\frac{2}{3}}$ and $F_{\omega L R}^{\frac{2}{3}}$ The full profiles are defined by $\mathrm{Eq}$ (C 98), where $a=u, \rho, \sigma, \tau, \omega$

\section{C.4 Vertices}

In the following we provide the relevant vertices for our calculations It should be noted that the final forms given are derıved with respect to the 4D particle fields - and therefore will contain the profiles of the associated particles - but are not integrated over the fifth dimension The effective 4D couplings can be found by integrating these forms over the fifth dimension, while 5D couplings can be obtained by dropping the interacting particles' profiles

\section{C.4.1 4-particle interaction terms: $W^{3_{L R}} W^{3_{L} R} H H$}

For the interaction $f \bar{f} \rightarrow Z H$ we would like to determine all couplings of form (gauge boson $) \rightarrow \mathrm{ZH}$ As the $\mathrm{Z}$ boson will arise from $A_{\mu}^{3_{L} R} \rightarrow W_{\mu}^{3_{L R}}$ as in the SM and the Higgs will arise from the fifth component of one of the $\mathrm{SO}(5) / \mathrm{SO}(4)$ gauge bosons $\left(A_{5}^{a}\right)$, we must consider all Lagrangian terms proportional to $A_{\nu}^{3_{L} R} A_{\mu}^{3_{L} R} A_{5}^{a} A_{5}^{a} 5$ Starting with the same Lagrangıan as in Eq (38), we take only the term of $F_{M R} F_{N S}$ involving a product of commutators, and let $M, N=\mu, \nu$ and $R, S=5$

$$
L_{4 v}=-\frac{\sqrt{g}}{2} g_{5}^{2} g^{\mu \nu} g^{55} A_{\mu 5}^{a} A_{\nu 5}^{a}=\frac{1}{2 k z} g_{5}^{2} \eta^{\mu \nu} A_{\mu 5}^{a} A_{\nu 5}^{a}
$$

\footnotetext{
${ }^{5}$ Note that the metric factors prevent couplings of form $A_{\mu} A_{\nu} A_{\alpha} A_{5}$ or $A_{\mu} A_{5} A_{5} A_{5}$ Therefore an $A_{5}$ cannot be the propagator in an interaction such as $e^{+} e^{-} \rightarrow Z H$
} 
From an inspection of Eq (B 6-B 9), we can see that we must consider only the $a=\hat{3}$ term, as it is the only one that contains terms with one $A_{5}^{4}$ field and one $A_{\mu}^{3_{L} R}$ field We find that

$$
\begin{aligned}
L_{4 v} \supset & \frac{1}{8 k z} g_{5}^{2} \eta^{\mu \nu} A_{5}^{4} A_{5}^{4}\left(W_{\mu}^{3_{L}} W_{\nu}^{3_{L}}-2 W_{\mu}^{3_{L}} W_{\nu}^{3_{R}}+W_{\mu}^{3_{R}} W_{\nu}^{3_{R}}\right) \\
= & \frac{1}{8 k z} g_{5}^{2} \eta^{\mu \nu}\left[H_{5}(x, z)+v f_{H}(z)\right]^{2}\left(W_{\mu}^{3_{L}} W_{\nu}^{3_{L}}-2 W_{\mu}^{3_{L}} W_{\nu}^{3_{R}}+W_{\mu}^{3_{R}} W_{\nu}^{3_{R}}\right) \\
= & \frac{1}{8 k z} g_{5}^{2} \eta^{\mu \nu}\left(W_{\mu}^{3_{L}} W_{\nu}^{3_{L}}-2 W_{\mu}^{3_{L}} W_{\nu}^{3_{R}}+W_{\mu}^{3_{R}} W_{\nu}^{3_{R}}\right) \\
& \times\left(H_{5}(x, z)^{2}+2 H_{5}(x, z) v f_{H}(z)+v^{2} f_{H}(z)^{2}\right)
\end{aligned}
$$

Therefore the relevant terms for $W_{\mu}^{3_{L} R} W_{\nu}^{3_{L} R} h_{5}$ interactions are

$$
L_{4 v} \supset \frac{1}{4 k z} g_{5}^{2} \eta^{\mu \nu} v f_{H}(z) H_{5}(x, z)\left(W_{\mu}^{3_{I}} W_{\nu}^{3_{L}}-2 W_{\mu}^{3_{L}} W_{\nu}^{3_{R}}+W_{\mu}^{3_{R}} W_{\nu}^{3_{R}}\right)
$$

Substıtuting Eqs (2 14-2 15), Eqs (3 14315 ), and Eqs (C 117) into Eq (C 115), we find the following vertex between two 5D Higgs bosons $H_{5}$ and two 5D photons,

$$
H_{5} H_{5} V_{\mu} V^{\mu} \rightarrow \frac{1}{2 k z}\left(\sin ^{2} \theta_{w}-2 \sin \theta_{w} \cos \theta_{H} \cos \theta_{w}+\cos ^{2} \theta_{w} \cos ^{2} \theta_{H}\right)
$$

As the Higgs is neutral, we require that this vertex be zero This condition results in the following relationship between $\theta_{H}$ and the Weinberg angle $\theta_{w}$,

$$
\cos \theta_{H}=\tan \theta_{w}
$$

This fixes $B_{\mu}$ and $X_{\mu}$ in terms of $W_{\mu}^{3_{R}}$ and $U_{\mu}$ With this relationship, both the $H_{5} H_{5} A_{\mu} Z^{\mu}$ and $H_{5} H_{5} A_{\mu} X^{\mu}$ couplings also vanish The former is fortunate, as the $Z$ boson is known to be neutral, the latter tells us that the $X$ boson is also neutral Note that if we had chosen $A_{5}^{3}$ as our Higgs the relation between $\theta_{H}$ and $\theta_{w}$ would be the same, the choice of either $A_{5}^{1}$ or $A_{5}^{2}$ simply change the relation by a minus sign, $\cos \theta_{H}=-\tan \theta_{w}$ 
Applying this relation, we fimally find

$$
L_{4 v} \supset \frac{g_{5}^{2}}{4 k z} \eta^{\mu \nu} v f_{H}(z) h_{5}(x, z)\left(\frac{1}{\cos ^{2} \theta_{w}} Z_{\mu} Z_{\nu}+\frac{2 \sqrt{\cos 2 \theta_{w}}}{\cos ^{2} \theta_{w}} X_{\mu} Z_{\nu}+\frac{\cos 2 \theta_{w}}{\cos ^{2} \theta_{w}} X_{\mu} X_{\nu}\right)
$$

Decomposing the 5D fields into 4D fields with profiles, taking the functional derivative of each term, and multiplyıng by $\imath$, we obtain the Feynman rules for the relevant 4D vertices,

$$
\begin{aligned}
Z_{\mu}^{(n)} Z_{\mu}^{(m)} H & \rightarrow \frac{\imath g_{z}^{2}}{2 k z} \frac{g_{5}}{g} \eta^{\mu \nu} v f_{H}(z)^{2} f_{Z}^{(n)}(z) f_{Z}^{(m)}(z) \\
X_{\mu}^{(n)} Z_{\mu}^{(m)} H & \rightarrow \frac{\imath g_{z} g_{x}}{2 k z} \frac{g_{5}}{g} \eta^{\mu \nu} v f_{H}(z)^{2} f_{X}^{(n)}(z) f_{Z}^{(m)}(z) \\
X_{\mu}^{(n)} X_{\mu}^{(m)} H & \rightarrow \frac{\imath g_{x}^{2}}{2 k z} \frac{g_{5}}{g} \eta^{\mu \nu} v f_{H}(z)^{2} f_{X}^{(n)}(z) f_{X}^{(m)}(z)
\end{aligned}
$$

where we have defined

$$
g_{z}=\frac{g}{\cos \theta_{w}} \quad \text { and } \quad g_{x}=\frac{\sqrt{\cos 2 \theta_{w}}}{\cos \theta_{w}}
$$

Note that if we integrate the $Z_{\mu}^{(0)} Z_{\mu}^{(0)} H(x, z)$ vertex

$$
\frac{g_{z}^{2} v}{2} \frac{\ln k L_{1}}{k} \eta^{\mu \nu} \int_{L_{0}}^{L_{1}} \frac{d z}{k z} f_{H}(z)^{2} f_{G}^{(0)}(z)^{2}=\frac{g_{z}^{2} v}{2} \eta^{\mu \nu} \int_{L_{0}}^{L_{1}} d z z \frac{2}{L_{1}^{2}-L_{0}^{2}}=\frac{g_{z}^{2} v}{2} \eta^{\mu \nu}
$$

we obtain an effective $4 \mathrm{D}$ term that is the same as the corresponding SM term

\section{C.4.2 3-particle interaction: Three gauge bosons}

The interaction of three gauge bosons arıse from the terms in the gauge Lagrangian,

$\mathrm{Eq}$ (3 8), proportional to both a derivative of the gauge field and a commutation of 
gauge fields,

$$
\begin{aligned}
L_{3 v} & =-g_{5} \frac{\sqrt{g}}{4} g^{M N} g^{R S}\left[\left(\partial_{M} A_{R}^{a}\right) A_{N S}^{a}-\left(\partial_{R} A_{M}^{a}\right) A_{N S}^{a}+A_{M R}^{a}\left(\partial_{N} A_{S}^{a}\right)-A_{M R}^{a}\left(\partial_{S} A_{N}^{a}\right)\right] \\
& =-\frac{g_{5}}{2 k z} \eta^{\mu \nu} \eta^{\alpha \beta}\left[\left(\partial_{\mu} A_{\alpha}^{a}\right) A_{\nu \beta}^{a}-\left(\partial_{\alpha} A_{\mu}^{a}\right) A_{\nu \beta}^{a}\right]+\frac{g_{5}}{k z} \eta^{\mu \nu}\left[\left(\partial_{\mu} A_{5}^{a}\right) A_{\nu 5}^{a}-\left(\partial_{5} A_{\mu}^{a}\right) A_{\nu 5}^{a}\right] \\
& =-\frac{g_{5}}{k z} \eta^{\mu \nu}\left[\eta^{\alpha \beta}\left(\partial_{\mu} A_{\alpha}^{a}\right) A_{\nu \beta}^{a}-\left(\partial_{\mu} A_{5}^{a}\right) A_{\nu 5}^{a}+\left(\partial_{5} A_{\mu}^{a}\right) A_{\nu 5}^{a}\right] \\
& =-\frac{g_{5}}{k z} \eta^{\mu \nu}\left[\eta^{\alpha \beta}\left(\partial_{\mu} A_{\alpha}^{a}\right) A_{\nu \beta}^{a}+\left(\partial_{5} A_{\mu}^{a}\right) A_{\nu 5}^{a}\right]
\end{aligned}
$$

where we have used the fact that $A_{\mu \nu}^{a}=-A_{\nu \mu}^{a}$ The last step is attained by neglecting all fifth components other than the Higgs $(a=4)$, as they are either zero, or are Goldstone bosons that will be eaten to become longitudinal polarizations of the vector bosons

Let us consider the first term of Eq (C 124), kecping only terms involving bosons with zero modes for the moment This leaves only the interaction between $W_{\mu}^{1_{L}}, W_{\mu}^{2_{L}}$, and $W_{\mu}^{3_{L}}$,

$$
\begin{aligned}
L_{w_{1 L} w_{2 L} w_{3 L}} & =-\frac{g_{5}}{k z} \eta^{\mu \nu} \eta^{\alpha \beta}\left(\partial_{\mu} A_{\alpha}^{a_{L}}\right) A_{\nu \beta}^{a_{L}} \\
& =-\frac{g_{5}}{k z} \eta^{\mu \nu} \eta^{\alpha \beta} \epsilon^{a_{L} b_{L} c_{L}}\left(\partial_{\mu} A_{\alpha}^{a_{L}}\right) A_{\nu}^{c_{L}} A_{\beta}^{b_{L}}
\end{aligned}
$$

Makıng replacements ${ }^{6} A(x z)=\int d^{4} p \exp (-\imath p x) A(p, z)$ in the corresponding action, decomposing the 5D fields into KK modes, taking the functional derivative [11], and multiplying by $\imath$ we obtain our Feynman rule for the vertex $W_{\mu}^{1_{L}}\left(k_{1}\right) W_{\nu}^{2_{L}}\left(k_{2}\right) W_{\lambda}^{3_{L}}\left(k_{3}\right)$

$$
\begin{aligned}
W_{\mu}^{1_{L}(n)}\left(p_{1}\right) W_{\nu}^{2_{L}(m)}\left(p_{2}\right) W_{\lambda}^{3_{L}(l)}\left(p_{3}\right) & \rightarrow \frac{g_{5}}{k z}\left[\eta^{\mu \lambda}\left(p_{3}^{\nu}-p_{1}^{\nu}\right)+\eta^{\mu \nu}\left(p_{1}^{\lambda}-p_{2}^{\lambda}\right)\right. \\
& \left.+\eta^{\nu \lambda}\left(p_{2}^{\mu}-p_{3}^{\mu}\right)\right] f_{1_{L}}^{(n)}(z) f_{2_{L}}^{(m)}(z) f_{3_{L}}^{(l)}(z)
\end{aligned}
$$

\footnotetext{
${ }^{6}$ Here we use the convention that the momenta $p$ are incoming
} 
where we use the convention that all particle momenta are incoming This is the same vertex as found in the SM, except for the profiles, the factor of $(k z)^{-1}$ and the replacement $g \rightarrow g_{5}$ [11] The rotation from this basis to the physical basis, $W_{\mu}^{1_{L}} W_{\nu}^{2_{L}} W_{\alpha}^{3_{L}} \rightarrow W_{\mu}^{+} W_{\nu}^{-} Z_{\alpha}$, works the same as way as in the SM, except that now the profiles are rotated with the $4 \mathrm{D}$ fields, as such, we find that in the physical basis

$$
\begin{aligned}
W_{\mu}^{+}\left(k_{+}\right) W_{\nu}^{-}\left(k_{-}\right) Z_{\lambda}(q) \rightarrow & \frac{2 g_{z}}{k z} \cos ^{2} \theta_{w} \frac{g_{5}}{g}\left[\eta^{\mu \nu}\left(k_{+}^{\lambda}-k_{-}^{\lambda}\right)+\eta^{\nu \lambda}\left(k_{-}^{\mu}-q^{\mu}\right)\right. \\
& \left.+\eta^{\lambda \mu}\left(q^{\nu}-k_{+}^{\nu}\right)\right] f_{W^{+}}^{(n)}(z) f_{W^{-}}^{(m)}(z) f_{Z}^{(l)}(z)
\end{aligned}
$$

If we consider all vertices in the first term of Eq (C 124) - not simply those corresponding to the SM - we find that the vertices will all have the same form as Eq (C 127), with the following replacements of the indices

$$
\begin{aligned}
2\left(a_{L, R}, \hat{b}, \hat{c}\right) \rightarrow & 2\left(1_{R}, 2_{R}, 3_{R}\right),\left(1_{L}, \hat{1}, \hat{4}\right),\left(1_{L}, \hat{2}, \hat{3}\right),\left(2_{L}, \hat{3}, \hat{1}\right), \\
& \left(2_{L}, \hat{2}, \hat{4}\right),\left(3_{L}, \hat{1}, \hat{2}\right),\left(3_{L}, \hat{3}, \hat{4}\right),-\left(1_{R}, \hat{1}, \hat{4}\right),\left(1_{R}, \hat{2}, \hat{3}\right), \\
& \left(2_{R}, \hat{3}, \hat{1}\right),-\left(2_{R}, \hat{2}, \hat{4}\right),\left(3_{R}, \hat{1}, \hat{2}\right),-\left(3_{R}, \hat{3}, \hat{4}\right),
\end{aligned}
$$

where outer factors $(-, 2)$ denote a difference of this overall factor in the vertex structure In the physical basis, the coupling of three gauge bosons $G_{1}, G_{2}$ and $G_{3}$ will be given by Eq (C 127) with the coefficient replacement $g \rightarrow C_{G_{1} G_{2} G_{3}}$ - where $C_{G_{1} G_{2} G_{3}}$ is a function of $\theta_{w}$ proportional to $g$ - and the profile replacement

$$
f_{1_{L}}^{(n)}(z) f_{2_{L}}^{(m)}(z) f_{3_{L}}^{(l)}(z) \rightarrow f_{G_{1}}^{(n)}(z) f_{G_{2}}^{(m)}(z) f_{G_{3}}^{(l)}(z)
$$

The specific coefficients $C_{G_{1} G_{2} G_{3}}$ for the couplings relevant to our purposes are

$$
\begin{gathered}
C_{Z W_{L}^{+} W_{L}^{-}}=\imath g_{z} \cos ^{2} \theta_{w}, C_{Z W_{R} W_{R}}=-\imath g_{z} \sin ^{2} \theta_{w}, \quad C_{Z A^{ \pm} A^{\mp}}=\frac{\imath}{2} g_{z} \cos 2 \theta_{w}, \\
C_{Z A^{3} A^{4}}=\frac{g_{z}}{2}, C_{X W_{R}^{+} W_{R}}=-\imath g_{x} \quad C_{X A^{ \pm} A^{\mp}}=-\frac{\imath}{2} g_{x}, C_{X A^{3} A^{4}}=\frac{g_{x}}{2} \\
C_{W_{L}^{\mp} A^{ \pm} A^{3}}=C_{W_{R}^{\mp} A^{\mp} A^{3}}= \pm \frac{\imath}{2}, \quad C_{W_{L}^{\mp} A^{ \pm} A^{4}}=-C_{W_{R}^{\mp} A^{ \pm} A^{3}}= \pm \frac{1}{2}
\end{gathered}
$$


If we now consider the second term of $\mathrm{Eq} \mathrm{C} 124$ ), we find four fur ther couplings involving the Higgs boson of form $A_{\mu}^{a_{I} R} A_{\nu}^{a} H_{5}$

$$
\begin{array}{r}
L_{A^{a_{L} R} A^{a} H}=-\frac{g_{5}}{k z} \eta^{\mu \nu}\left(\partial_{5} A_{\mu}^{a}\right) A_{\nu 5}^{a}=-\frac{g_{5}}{2 k z} \eta^{\mu \nu} \sum_{a=1}^{3}\left[\left(\left(\partial_{z} A_{\mu}^{a_{L}}\right) A_{\nu}^{a}-\left(\partial_{z} A_{\mu}^{a}\right) A_{\nu}^{a_{L}}\right) h_{5}\right. \\
\left.-\left(\left(\partial_{z} A_{\mu}^{a_{R}}\right) A_{\nu}^{a}-\left(\partial_{z} A_{\mu}^{a}\right) A_{\nu}^{a_{R}}\right) h_{5}\right], \quad(\mathrm{C} 132)
\end{array}
$$

where we have kept only terms where $A_{5}^{a}=A_{5}^{4} \quad$ As we are primarly interested in the couplings involving $Z$ and $X$ bosons, let's concentrate on the $a=3$ term Transforming to the physical basis, we find that the couplings of the $4 \mathrm{D}$ field components are

$$
\begin{aligned}
G_{\mu}^{(n)} A_{\nu}^{a(m)} H \rightarrow \frac{\imath}{2 k z} C_{G A^{a} H} \frac{g_{5}}{g} f_{H}(z) & {\left[\left(\partial_{z} f_{G}^{(n)}(p, z)\right) f_{A}^{(m)}(p, z)\right.} \\
& \left.-\left(\partial_{z} f_{A}^{(m)}(p, z)\right) f_{G}^{(n)}(p, z)\right],
\end{aligned}
$$

where $G=Z, X, W^{ \pm}, \hat{a}=+,-, \hat{3}$ and the particular coefficients are

$$
C_{Z A^{3} H}=g_{z}, \quad C_{X A^{3} H}=g_{x} \quad C_{W_{L}^{ \pm} A \top H}=-C_{W_{R}^{ \pm} A^{\mp} H}=g
$$

It is worth noting that in the absence of particle mixing all of the corresponding $4 \mathrm{D}$ effective couplings of this form will be zero if the relevant $Z, X$ or $W$ boson is a zero mode, as the profiles will integrate to zero

$$
\begin{aligned}
& \frac{g_{G}}{2} \frac{g_{5}}{g} \int d z \frac{1}{k z} f_{H}(z)\left[\left(\partial_{z} f_{G}^{(0)}\left(m_{G}\right)\right) f_{A}^{(m)}\left(m_{A}, z\right)-\left(\partial_{z} f_{A}^{(m)}\left(m_{A}, z\right)\right) f_{G}^{(0)}\left(m_{G}\right)\right] \\
& =-\frac{g_{G}}{2 k} \frac{g_{5}}{g} f_{G}^{(0)}\left(m_{G}\right) \sqrt{\frac{2 k}{L_{1}^{2}-L_{0}^{2}}} \int d z\left(\partial_{z} f_{A}^{(m)}\left(m_{4}, z\right)\right) \\
& =-\frac{g_{G}}{2 k} \frac{g_{5}}{g} f_{G}^{(0)}\left(m_{G}\right) \sqrt{\frac{2 k}{L_{1}^{2}-L_{0}^{2}}}\left[f_{A}^{(m)}\left(m_{A}, L_{1}\right)-f_{A}^{(m)}\left(m_{A}, L_{0}\right)\right] \\
& =0
\end{aligned}
$$


by the Dirichelet boundary conditions on the coset bosons. Here we have used the fact that $f_{G}^{(0)}(z)$ is constant in $z$ and required that the particles be on-shell. The fact that these couplings are zero provides a second reason - beyond the lack of fermion anti-fermion couplings - why the propagator in $e^{+} e^{-} \rightarrow Z^{(0)} H$ cannot be an $A^{\hat{a}}$ boson. However, note that these zero-mode 3-particle couplings reappear after mixing because the zero-mode gauge boson profiles are no longer flat.

\section{C.4.3 3-particle interaction: Fermion-fermion-boson}

By applying integration by parts to the derivative in the second term in Eq. (3.12), we find that the spin-connection contribution from Eq.(3.13) cancels out, while the derivative and boson terms double. Therefore the relevant terms for the coupling of two fermions to a $\mathrm{Z}$ or $\mathrm{X}$ gauge boson are

$$
\begin{aligned}
L_{\bar{f} f G} \supset \frac{g_{5}}{(k z)^{4}} \eta^{\mu \nu}\left[\bar{\Psi}_{L} \gamma_{\nu}\left(\tilde{T}^{3_{L}} W_{\mu}^{3_{L}}+\tilde{T}^{3_{R}} W_{\mu}^{3_{R}}\right) \Psi_{L}+\bar{\Psi}_{R} \gamma_{\nu}\left(\tilde{T}^{3_{L}} W_{\mu}^{3_{L}}+\tilde{T}^{3_{R}} W_{\mu}^{3_{R}}\right) \Psi_{R}\right] \\
\quad+\frac{g_{5}^{\prime \prime}}{(k z)^{4}} \eta^{\mu \nu}\left[\bar{\Psi}_{L} \gamma_{\nu} Q_{B-L} \Psi_{L} U_{\mu}+\bar{\Psi}_{R} \gamma_{\nu} Q_{B-L} \Psi_{R} U_{\mu}\right]
\end{aligned}
$$

where we have kept only the $3_{L, R}$ terms, and $g_{5}^{\prime \prime}=g^{\prime \prime} \frac{g_{5}}{g}$ is the $5 \mathrm{D}$ gauge coupling of $U(1)_{B-L}$, and defined

$$
\tilde{T}_{5}^{3_{L, R}}=A T_{5}^{3_{L, R}} A^{-1}, \quad \tilde{T}_{10}^{3_{L, R}}=B T_{10}^{3_{L}, R} B^{-1}
$$

The 5 and 10 representations of the relevant generators are defined in Appendix B; the basis transformation matrices $A$ and $B$ are defined in Appendix C.3.

We can relate $Q_{B-L}$ to the hypercharge $Y$ and the weak generator $T^{3_{R}}$ by requiring that the coupling of the hypercharge boson $B_{\mu}$ to fermions match that of the SM, $g^{\prime} Y \vec{\Psi}_{L, R} \gamma^{\mu} B_{\mu} \Psi_{L, R}$, where $g^{\prime}=g \tan \theta_{w}$. In our model $B_{\mu}$ is a mix of 
$U_{\mu}$ and $W_{\mu}^{3_{R}}$, therefore the corresponding term arises from the second two terms of Eq. (C.136),

$$
\begin{array}{r}
\frac{g_{5}}{(k z)^{4}} \eta^{\mu \nu} \bar{\Psi}_{L, R} \gamma_{\nu}\left[\tilde{T}^{3_{R}} W_{\mu}^{3_{R}}+g_{5}^{\prime \prime} Q_{B-L} U_{\mu}\right] \Psi_{L, R} \\
\supset \frac{\eta^{\mu \nu}}{(k z)^{4}} \bar{\Psi}_{L, R} \gamma_{\nu}\left[g_{5} \tilde{T}^{3_{R}} \cos \theta_{H}+g_{5}^{\prime \prime} Q_{B-L} \sin \theta_{H}\right] B_{\mu} \Psi_{L, R}
\end{array}
$$

If we consider the zero-modes, the $5 \mathrm{D}$ components will integrate to 1 (as shown in Eq.(C.144)). We therefore require that

$$
Y=\frac{g}{g^{\prime}} \tilde{T}^{3_{R}} \cos \theta_{H}+\frac{g^{\prime \prime}}{g^{\prime}} Q_{B-L} \sin \theta_{H}=\tilde{T}^{3_{R}}+Q_{B-L} .
$$

where we have made use of $g=\tan \theta_{H} g^{\prime \prime}$.

By making use of these relations as we substitute Eqs. (2.14-2.15) into Eq.(C.136) - and neglecting the photon fields - the vertex terms become

$$
\begin{aligned}
L_{\tilde{f} f G}^{(L, R)} \supset & \frac{g_{z}}{(k z)^{4}} \frac{g_{5}}{g} \bar{\Psi}_{L, R}\left(\tilde{T}^{3}{ }^{L}-Q \sin ^{2} \theta_{w}\right) \gamma^{\mu} Z_{\mu} \Psi_{L, R} \\
& +\frac{g_{x}}{(k z)^{4}} \frac{g_{5}}{g} \bar{\Psi}_{L, R}\left(\frac{\left(Q-\tilde{T}^{3_{L}}\right) \sin ^{2} \theta_{w}-\tilde{T}^{3_{R}} \cos ^{2} \theta_{w}}{\cos 2 \theta_{w}}\right) \gamma^{\mu} X_{\mu} \Psi_{L, R}
\end{aligned}
$$

where $Q=Y+\tilde{T}^{3_{L}}$ - the electromagnetic charge in units of $e$, not to be confused with $Q_{B-L}$ - is defined by requiring that the photon-fermion vertex be $Q e$. Note that if we take $\bar{\Psi}, \Psi$ to be specific fermions, rather than full fermion vectors, then $\tilde{T}^{3_{L, R}}$ denotes the scalar element of the generator matrix that selects the appropriate fermions from the fermion spinors. Although the vertices of the right- and left-handed fermions have the same mathematical form, the element of $\tilde{T}^{3_{L, R}}$ corresponding to each will not in general be the same; as such the right- and left-handed couplings will not have the same values, despite having the same form.

For right-handed SM particles, $T^{3_{L}}=0$. Therefore the fermion coupling to $Z$ bosons in Eq. (C.141) matches that of the Standard Model in both the left- and 
right-handed cases, as desired

$$
\begin{aligned}
L_{f f G}^{(L R)} \supset & \frac{g_{z}}{(k z)^{4}} \frac{g_{5}}{g} \bar{\Psi}_{L}^{(S M)}\left(\tilde{T}^{3 L}-Q \sin ^{2} \theta_{w}\right) \gamma^{\mu} Z_{\mu} \Psi_{L}^{(S M)} \\
& +\frac{g_{z}}{(k z)^{4}} \frac{g_{5}}{g} \bar{\Psi}_{R}^{(S M)}\left(-Q \sin ^{2} \theta_{w}\right) \gamma^{\mu} Z_{\mu} \Psi_{R}^{(S M)}
\end{aligned}
$$

Furthermore, note that for left-handed partıcles $T^{3_{I}}=T^{3_{R}}$, in this case the lefthanded $X$ coupling reduces to the same form as the $Z$ coupling, but with opposite sign and an inverse factor of $\cos 2 \theta_{w}$ This correspondence between the $Z$ and $X$ fermion couplings also holds in the case of $r$ ight-handed up-type fermions The correspondence is notable as the XZH vertex of the Higgsstrahlung interaction brings an extra factor of $g_{x}^{2}$, which cancels the extra cosinc factor and results in $Z$ and $X$ Higgsstrahlung coupling constants of equal but opposite magnitude

Because $A T^{3_{L R}} A^{-1}, B T_{10}^{3_{L} R} B^{-1}$ and the matrix form of $Q$ are all diagonal, $Z$ and $X$ can only couple a given fermion $f$ to its own anti-fermion $\bar{f}$ However, the gauge boson $A_{\mu}^{3}$ can couple different fermions of the same charge This will become relevant once the electroweak symmetry is broken because both the $X$ and $Z$ become a superposition of states that includes the $A_{\mu}^{3}$, allowing $\mathrm{KK}$ decays to $f_{1} \bar{f}_{2}$ The Lagrangian term corresponding to the coupling of a fermion antı-fermion pair to $A_{\mu}^{3}$ is simply given by

$$
L_{f f G} \supset \frac{g_{5}}{(k z)^{4}} \eta^{\mu \nu} \bar{\Psi}_{L} \gamma_{\nu} T^{3} A_{\mu}^{3} \Psi_{L}
$$

All of the possible couplings of $Z, X$ or $A_{\mu}^{3}$ bosons to fermions are summarized in Table C 1 


\begin{tabular}{|c|c|c|c|c|c|c|}
\hline Boson & Lepton pair & Quark pair & $Q_{\ell}, Q_{q}$ & $\tilde{T}^{3_{L}}$ & $\tilde{T}^{3_{R}}$ & $\tilde{T}^{\hat{3}}$ \\
\hline $\mathrm{Z}, \mathrm{X}$ & $\bar{\kappa}_{1} \kappa_{1}, \bar{\kappa}_{2} \kappa_{2}, \bar{\kappa}_{3} \kappa_{3}$ & $\bar{\chi}_{1} \chi_{1}, \bar{\chi}_{2} \chi_{2}, \bar{\chi}_{3} \chi_{3}$ & $1, \frac{5}{3}$ & $\frac{1}{2}$ & $\frac{1}{2}$ & 0 \\
\hline $\mathrm{Z}, \mathrm{X}$ & $\overline{\hat{\eta}}_{1} \hat{\eta}_{1}, \overline{\hat{\eta}}_{2} \hat{\eta}_{2}, \overline{\hat{\eta}}_{3} \hat{\eta}_{3}$ & $\overline{\hat{t}}_{1} \hat{t}_{1}, \overline{\hat{t}}_{2} \hat{t}_{2}, \overline{\hat{t}}_{3} \hat{t}_{3}$ & $0, \frac{2}{3}$ & $-\frac{1}{2}$ & $\frac{1}{2}$ & 0 \\
\hline $\mathrm{Z}, \mathrm{X}$ & $\bar{\eta}_{1} \eta_{1}, \bar{\eta}_{2} \eta_{2}, \bar{\eta}_{3} \eta_{3}$ & $\bar{t}_{1} t_{1}, \bar{t}_{2} t_{2}, \bar{t}_{3} t_{3}$ & $0, \frac{2}{3}$ & $\frac{1}{2}$ & $-\frac{1}{2}$ & 0 \\
\hline $\mathrm{Z}, \mathrm{X}$ & $\bar{\ell}_{1} \ell_{1}, \bar{\ell}_{2} \ell_{2}, \bar{\ell}_{3} \ell_{3}$ & $\bar{b}_{1} b_{1}, \bar{b}_{2} b_{2}, \bar{b}_{3} b_{3}$ & $-1,-\frac{1}{3}$ & $-\frac{1}{2}$ & $-\frac{1}{2}$ & 0 \\
\hline $\mathrm{Z}, \mathrm{X}$ & $\overline{\tilde{\eta}}_{1} \tilde{\eta}_{1}, \overline{\tilde{\eta}}_{2} \tilde{\eta}_{2}, \bar{N}_{3} N_{3}, \bar{N}_{3}^{\prime} N_{3}^{\prime}$ & $\overline{\tilde{t}}_{1} \tilde{t}_{1}, \overline{\tilde{t}}_{2} \tilde{t}_{2}, \bar{T}_{3} T_{3}, \bar{T}_{3}^{\prime} T_{3}^{\prime}$ & $0, \frac{2}{3}$ & 0 & 0 & 0 \\
\hline $\mathrm{Z}, \mathrm{X}$ & $\bar{K}_{3} K_{3}$ & $\Xi_{3} \Xi_{3}$ & $1, \frac{5}{3}$ & 1 & 0 & 0 \\
\hline $\mathrm{Z}, \mathrm{X}$ & $\bar{L}_{3} L_{3}$ & $\bar{B}_{3} B_{3}$ & $-1,-\frac{1}{3}$ & -1 & 0 & 0 \\
\hline $\mathrm{Z}, \mathrm{X}$ & $\bar{K}_{3}^{\prime} K_{3}^{\prime}$ & $\Xi_{3}^{\prime} \Xi_{3}^{\prime}$ & $1, \frac{5}{3}$ & 0 & 1 & 0 \\
\hline $\mathrm{Z}, \mathrm{X}$ & $\bar{L}_{3}^{\prime} L_{3}^{\prime}$ & $\bar{B}_{3}^{\prime} B_{3}^{\prime}$ & $-1,-\frac{1}{3}$ & 0 & -1 & 0 \\
\hline$A_{\mu}^{\hat{3}}$ & $\overline{\tilde{\eta}}_{3} N_{3}^{\prime}, \bar{N}_{3} \eta_{3}+$ c.c & $\overline{\tilde{t}}_{3} T_{3}^{\prime}, \bar{T}_{3} t_{3}+$ c.c & $0, \frac{2}{3}$ & 0 & 0 & $-\frac{\sqrt{2} 2}{4}$ \\
\hline$A_{\mu}^{\hat{3}}$ & $\overline{\tilde{\eta}}_{3} N_{3}, \bar{N}_{3}^{\prime} \eta_{3}+$ c.c & $\overline{\tilde{t}}_{3} T_{3}, \bar{T}_{3}^{\prime} t_{3}+$ c.c & $0, \frac{2}{3}$ & 0 & 0 & $\frac{\sqrt{22}}{4}$ \\
\hline$A_{\mu}^{\hat{3}}$ & $\bar{\ell}_{3} L_{3}^{\prime}, \bar{\ell}_{3} L_{3}+$ c.c & $\bar{b}_{3} B_{3}^{\prime}, \bar{b}_{3} B_{3}+$ c.c & $-1,-\frac{1}{3}$ & 0 & 0 & $-\frac{2}{2}$ \\
\hline$A_{\mu}^{\hat{3}}$ & $\bar{\kappa}_{3} K_{3}^{\prime}, \bar{\kappa}_{3} K_{3}+$ c.c & $\bar{\chi}_{3} \Xi_{3}^{\prime}, \bar{\chi}_{3} \Xi_{3}+$ c.c & $1, \frac{5}{3}$ & 0 & 0 & $\frac{\imath}{2}$ \\
\hline$A_{\mu}^{\hat{3}}$ & $\overline{\tilde{\eta}}_{1} \hat{\eta}_{1}, \overline{\tilde{\eta}}_{2} \hat{\eta}_{2}+$ c.c & $\overline{\tilde{t}}_{1} \hat{t}_{1}, \overline{\tilde{t}}_{2} \hat{t}_{2}+$ c.c & $0, \frac{2}{3}$ & 0 & 0 & $-\frac{1}{2}$ \\
\hline$A_{\mu}^{\hat{3}}$ & $\bar{\eta}_{1} \hat{\eta}_{1}, \bar{\eta}_{2} \hat{\eta}_{2}+$ c.c & $\bar{t}_{1} \hat{t}_{1}, \bar{t}_{2} \hat{t}_{2}+$ c.c & $0, \frac{2}{3}$ & 0 & 0 & $\frac{1}{2}$ \\
\hline
\end{tabular}

Table C.1: Possible couplings of $Z, X$ or $A_{\mu}^{\hat{3}}$ bosons to lepton anti-lepton pairs. The $L, R$ subscripts have becn suppressed. Refer to Eqs. (3.23-3.28) for the multiplet structure. 


\section{C.5 The Relation Between $g$ and $g_{5}$}

The relation between the 5D gaugc coupling $g_{5}$ and the familiar SM gauge coupling $g$ can be determined by comparing effective 4D MCHM couplings to those of the SM It should be noted that particle mixing will alter the profiles used in the derivation below, and therefore cause a shift in the relationship between $g$ and $g_{5}$ However, as zero modes are not substantially altered by mixıng this shift will be quite small

\section{C.5.1 From the four gauge boson vertex}

To determine the relation between the 4D SM gauge coupling $g$ and our 5D coupling constant $g_{5}$, consider a vertex of four gauge bosons Proceeding similarly to $\mathrm{Eq}$ (C 17), and neglecting all brane-restricted contributions to the Lagrangian, we must obtain $g^{2}$ when all $z$ dependent contributions and the $g_{5}^{2}$ factor are integrated over $z$ Therefore we require that

$$
g^{2}=\int_{L_{0}}^{L_{1}} d z \frac{g_{5}^{2}}{k z}\left[f_{G}^{(0)}(z)\right]^{4}=\frac{g_{5}^{2}}{k}\left[\frac{k}{\ln \left(k L_{1}\right)}\right]^{2} \int_{L_{0}}^{L_{1}} d z \frac{1}{z}=\frac{k g_{5}^{2}}{\ln \left(k L_{1}\right)}
$$

If brane terms were included the relation would have form

$$
\frac{1}{g^{2}}=\frac{\ln \left(k L_{1}\right)}{k g_{5}^{2}}+\frac{1}{g_{\mathrm{UV}}}+\frac{1}{g_{\mathrm{IR}}}
$$

where $g_{\mathrm{UV}}$ and $g_{\mathrm{IR}}$ are possible contributions from the UV and IR brane terms [3], which are zero in our case

\section{C.5.2 From a $\bar{f} f A_{\mu}$ vertex}

The coupling constant relation can also be derıved by considering a vertex where a fermion and anti-fermion couple to a gauge boson Specifically, let's consider $W_{L}^{+} \rightarrow$ 
$e^{-} \bar{\nu}_{L} \quad$ As before, the $4 \mathrm{D}$ constant $g$ must be equal to the integral of all the $5 \mathrm{D}$ components Using Eq (C 39), Eq (C 25) and noting that both fermions have the same associated parameter $c$ as they arc part of the same $S U(2)_{L}$ doublet, we have

$$
\begin{aligned}
g & =g_{5} \int_{L_{0}}^{L_{1}} d z \sqrt{g} k z f_{e}^{(0)}(z) f_{\nu}^{(0)}(z) f_{W}^{(0)}(z) \\
& =g_{5} \int_{L_{0}}^{L_{1}} d z \frac{1}{(k z)^{4}} \sqrt{\frac{k}{\ln \left(k L_{1}\right)} \frac{k(1-2 c)}{\left(k L_{1}\right)^{1-2 c}-1}(k z)^{4-2 c}} \\
& =g_{5} \frac{k(1-2 c)}{\left(k L_{1}\right)^{1-2 c}-1} k \sqrt[2 c]{\frac{k}{\ln \left(k L_{1}\right)}} \int_{L_{0}}^{L_{1}} d z z^{-2 c} \\
& =g_{5} \frac{1-2 c}{\left(k L_{1}\right)^{1-2 c}-1} k^{1-2 c} \frac{1}{1-2 c}\left(L_{1}^{1-2 c}-L_{0}^{1-2 c}\right) \sqrt{\frac{k}{\ln \left(k L_{1}\right)}} \\
& =g_{5} \sqrt{\frac{k}{\ln \left(k L_{1}\right)}},
\end{aligned}
$$

which is the same relation as found before 


\section{Appendix D}

\section{Gauge Boson Decay Widths}

To account for the physical widths of the KK resonances in the cross section, we will need the total decay rate of each propagating boson. In the following formulae, all constant and 5D integral factors of the couplings will be simply expressed as overall couplings $C$, as when full particle mixing is implemented the couplings constants and $5 \mathrm{D}$ integrals can no longer be separated. The appropriate couplings can be found in Appendix C.4.

\section{D.1 Boson decay to fermion final states}

The decay rate in the center-of-mass frame for a gauge boson $G=Z, X$ of mass $M_{G}$ to two fermions of mass $m_{1}$ and $m_{2}$ is given by

$$
\begin{array}{r}
\Gamma_{G f f}=\frac{\lambda^{\frac{1}{2}}\left(M_{G}^{2}, m_{1}^{2}, m_{2}^{2}\right)}{48 \pi M_{G}^{5}}\left[\left(C_{G f f}^{L 2}+C_{G f f}^{R 2}\right) \beta\left(M_{G}^{2}, m_{1}^{2}, m_{2}^{2}\right)\right. \\
\left.+12 M_{G}^{2} m_{1} m_{2} C_{G f f}^{L} C_{G f f}^{R}\right]
\end{array}
$$


where $M_{z}^{(n)}$ 1s the mass of the Z KK mode in question, $C_{G f f}^{L R}$ are the appropriate overall left- and right-handed couplings including 5D integral factors - and

$$
\begin{aligned}
& \lambda(x, y, z)=x^{2}+y^{2}+z^{2}-2 x y-2 x z-2 y z \\
& \beta(x, y, z)=2 x^{2}-y^{2}-z^{2}-x y-x z+2 y z
\end{aligned}
$$

In the case where both fermions are zero modes and their masses may be neglected $\left(m_{1}=m_{2}=0\right)$, this reduces to

$$
\Gamma_{G f f 0}=\frac{M_{G}}{24 \pi}\left[\left(C_{G f f}^{L 2}+C_{G f f}^{R 2}\right)\right]
$$

Note that for quarks these formulae will be multiphed by three to account for the different colours

\section{D.2 Gauge boson decay to a Higgs final state}

The decay-rate in the center-of-mass frame of a gauge boson $\mathrm{KK}$ mode $G_{1}=Z, X$ of order $n$ and mass $M_{1}$ to a second gauge boson $\mathrm{KK}$ mode $G_{2}=Z, X$ of order $m$ and mass $M_{2}$ and a Higgs boson is given by

$\Gamma_{G_{1} G_{2} H}^{(n, m)}=\frac{C_{G_{1} G_{2} H}}{48 \pi M_{1}^{(n) 3}} \lambda^{2}\left(M_{1}^{(n) 2}, M_{2}^{(m) 2}, M_{H}^{2}\right)\left[2+\frac{\left(M_{1}^{(n) 2}+M_{2}^{(m) 2}-M_{H}^{2}\right)^{2}}{4 M_{1}^{(n) 2} M_{2}^{(m) 2}}\right]$

where $M_{H}$ is the mass of the Higgs boson, $\lambda(x, y, z)$ is defined in Eq (D 2), and $C_{G_{1} G_{2} H}$ is the appropriate coupling constant of the two gauge bosons to the Higgs Here $n$ cannot be smaller than $m$, this is kinematically forbidden 


\section{D.3 Gauge boson decay to two other gauge bosons}

The decay rate in the centcr-of-mass frame of a gauge boson $G_{1}$ of order $n$ and mass $M_{1}$ to two other gauge bosons $G_{2}, G_{3}$ of orders $m, l$ and masses $M_{2}, M_{3}$ respectively, is given by

$$
\Gamma_{G_{1} G_{2} G_{3}}^{(n, m, l)}=\frac{C_{G_{1} G_{2} G_{3}}}{48 \pi M_{1}^{(n) 3}} \lambda^{\frac{1}{2}}\left(M_{1}^{(n) 2}, M_{2}^{(m) 2}, M_{3}^{(l) 2}\right) N\left(M_{1}^{(n)}, M_{2}^{(m)}, M_{3}^{(l)}\right)
$$

where $C_{G_{1} G_{2} G_{3}}$ is the appropriate coupling coefficient and

$$
\begin{aligned}
N(x, y, z)= & -8\left(x^{2}+y^{2}+z^{2}\right)+2\left(\frac{x^{4}}{y^{2}}+\frac{x^{4}}{z^{2}}+\frac{y^{4}}{x^{2}}+\frac{y^{4}}{z^{2}}+\frac{z^{4}}{x^{2}}+\frac{z^{4}}{y^{2}}\right) \\
& +\frac{1}{4}\left(\frac{x^{6}}{y^{2} z^{2}}+\frac{y^{6}}{x^{2} z^{2}}+\frac{z^{6}}{y^{2} x^{2}}\right)-\frac{9}{2}\left(\frac{y^{2} z^{2}}{x^{2}}+\frac{x^{2} z^{2}}{y^{2}}+\frac{y^{2} x^{2}}{z^{2}}\right) .
\end{aligned}
$$

It it is useful to know that when $y=z, N(x, y, z)$ reduces to

$$
N(x, y, y)=-12 y-17 x+4 \frac{x^{4}}{y^{2}}+\frac{x^{6}}{4 y^{4}}
$$




\section{Appendix E}

\section{Analytic integrals for ECA cross-section calculations}

E.1 Performing the integrations in the $5 \mathrm{D}$ coupling factor $Z_{G}(c, q)$

To perform the integrals, we must first substitute in Eqs. (C.144), (C.42), (C.39), (C.25) and (C.7), obtaining

$$
\begin{aligned}
Z_{G}(c, q)=\operatorname{Coef}(p) \int_{L_{0}}^{L_{1}} d z z^{1 \mp 2 c}\left[C_{G} J_{\alpha}(p z)+D_{G} Y_{\alpha}(p z)\right] \\
\times \int_{L_{0}}^{z} d z^{\prime} z^{\prime 2}\left[A_{G} J_{\alpha}\left(p z^{\prime}\right)+B_{G} Y_{\alpha}\left(p z^{\prime}\right)\right] \\
+\operatorname{Coef}(\mathrm{p}) \int_{L_{0}}^{L_{1}} d z z^{1 \mp 2 c}\left[A_{G} J_{\alpha}(p z)+B_{G} Y_{\alpha}(p z)\right] \\
\times \int_{z}^{L_{1}} d z^{\prime} z^{2}\left[C_{G} J_{\alpha}\left(p z^{\prime}\right)+D_{G} Y_{\alpha}\left(p z^{\prime}\right)\right]
\end{aligned}
$$




$$
\text { where } \quad \operatorname{Coef}(p)=\pi \frac{k^{3 \mp 2 c}}{A_{G} D_{G}-B_{G} C_{G}} \frac{1 \mp 2 c}{\left(k L_{1}\right)^{1 \mp 2 c}-1} \frac{\ln \left(k L_{1}\right)}{\left(k L_{1}\right)^{2}-1}
$$

where the minus applies to the cases with left-handed fermions and the plus applies to the cases with right-handed fermions, as the former have positive $c$ values while the latter have negative $c$ values. Coefficients $A, B, C$, and $D$ with $Z$ subscripts are defined by Neumann-Neumann boundary conditions, while those with $X$ subscripts are defined by Dirichlet-Neumann boundary conditions.

The integrals over $z^{\prime}$ can easily be solved using the basic properties of Bessel functions,

$$
\int d z z^{2} J_{1}(q z)=\frac{z^{2}}{q} J_{2}(q z), \quad \int d z z^{2} Y_{1}(q z)=\frac{z^{2}}{q} Y_{2}(q z)
$$

The integration over $z$ is complicated both by the variable $c$ - which is not necessarily an integer or fraction - and the multiplication of Bessel functions in the integrand. Terms that involve only one Bessel function can be solved using the Meijer G function $M_{G}\left(a_{s} ; b_{s} ; c_{s} ; d_{s} ; z\right)$ and the first Lommel function $L_{1}(a, b, z)$. For certain values of $c$ - those for which $1 \mp 2 c$ is an integer these functions simplify to yield results in terms of the Bessel functions and the Struve function $H_{\nu}(z)$. These special functions are defined in Appendix A and are incorporated as commands in programs such as Mathematica and Maple.

The single-Bessel integrals are given in general by

$$
\begin{gathered}
\int d z z^{1 \mp 2 c} J_{1}(z q)=q^{-1 \pm 2 c} z\left[(1 \mp 2 c) J_{1}(q z) L_{1}(\mp 2 c, 0, q z)\right. \\
\left.-J_{0}(q z) L_{1}(1 \mp 2 c, 1, q z)\right] \\
\int d z z^{1 \mp 2 c} Y_{1}(z q)=2^{1 \mp 2 c} q^{-2 \pm 2 c} M_{G}\left(1 ; \pm c ; 1 / 2 \mp c, 3 / 2 \mp c ; 0,-c, 1 / 4 z^{2} q^{2}\right) .
\end{gathered}
$$


The integrals involving two Bessel functions cannot always be integrated analytically. Those involving combinations of $J_{n}(x) Y_{m}(x)$ and $Y_{n}(x) Y_{m}(x)$ must be performed numerically. However, the integral involving two Bessel functions of the first kind can be expressed in terms of the generalized hypergeometric function $F\left(a_{s} ; b_{s}, z\right)$,

$$
\int d z z^{1 \mp 2 c} J_{1}(z q) J_{2}(z q)=\frac{q^{3} z^{5 \mp 2 c}}{16(5 \mp 2 c)} F\left(5 / 2,5 / 2 \mp c ; 3,4,7 / 2 \mp c ;-z^{2} q^{2}\right) \text {. }
$$




\section{References}

[1] L Randall and R Sundrum, "A largo mass herarchy from a small extra dimension," Phys Rev Lelt, vol 83, pp 3370 3373, 1999, hep-ph/9905221

[2] J M Maldacena, "The large N linnt of superconformal field theories and supergravity," Adv Theor Math Phys, vol 2, pp 231-252, 1998, hep-th/9711200

[3] K Agashe, R Contıno, and A Pomaıol, "The Minımal Composite Higgs Model," Nucl Phys, vol B719, pp 165 187, 2005, hep-ph/0412089

[4] A D Medina, N R Shah, and C E M Wagner, "Gauge-higgs unification and radiatıve electroweak symmetry breahıng in warped extra dımensıons," Phys Rev D, vol 76, p 095010, Nov 2007

[5] P Langacker, "Structure of the standard model," 1995, hep-ph/0304186

[6] P D Group, Partıcle Physıcs Booklet Elsevier, 2010

[7] G Marchesinı, "QCD review," Int J Mod Phys, vol A22, pp 5443 5452, 2007, hep-ph/0611115

[8] R Feynman, QED The strange theory of light and matter Princeton, NJ Princeton University Press 
[9] E Noether, "Invarıant Varıation Problems," Nachr d Konıg Gesellsch d Wiss zu Gottingen, Math-phys Klasse, pp 235 257, 1918 [An englush translation by M A Tavel can be found at arXıv hep-ph/0601213v1]

[10] S Dawson, "Introduction to electroweak symmetry breakıng," 1998, hep-ph/9901280

[11] M E Peskin and D V Shroeder, An Introduction to Quantum Field Theory Boulder, CO Westview Press, 1995

[12] J Goldstone, "Field theories with superconductor solutions," Il Nuovo Cimento (1955-1965), vol 19, pp 154 164, 196110 1007/BF02812722

[13] P W Hıggs, "Broken Symmetries Massless Partıcles and Gauge Fields," Physics Letters, vol 12 pp 132 133, Sept 1964

[14] P W Higgs, "Broken symmetries and the masses of gauge bosons," Phys Rev Lett, vol 13, pp 508-509, Oct 1964

[15] D Griffiths, Introduction to Elementary Partıcles Weinheim, Germany Wiley-VCH

[16] R Barate et al, "Search for the standard model Higgs boson at LEP," Phys Lett, vol B565, pp 61 75, 2003, hep-ex/0306033

[17] "Precision electroweak measurements on the Z resonance," Phys Rept, vol 427, pp 257-454, 2006, hep-ex/0509008

[18] T Aaltonen et al, "Inclusive Search for Standard Model Higgs Boson Production in the WW Decay Channel using the CDF II Detector," Phys Rev Lett, vol 104, p 061803, 2010, 10014468 
[19] T Aaltonen et al, "Combination of Tevatron searches for the standard model Higgs boson in the W+W-decay mode" Phys Rev Lett, vol 104, p 061802, 2010,10014162

[20] S P Martın, "A Supersymmetry Prımer," 1997, hep-ph/9709356

[21] J Kalınowskı, "SUSY Theory Review," Acta Physıca Polonıca B, vol 38, p 0531,2007

[22] M Schmaltz and D Tucker-Smith, "Little Higgs Review," Ann Rev Nucl Part Scr, vol 55, pp 229-270, 2005, hep-ph/0502182

[23] V A Mransky, M Tanabashı, and K Yamawakı, "Dynamıcal electroweak symmetry breakıng with large anomalous dimension and t quark condensate," Physics Letters B, vol 221, no 2, pp 177-183, 1989

[24] C T Hill, "Topcolor top quark condensation in a gauge extension of the standard model," Physıcs Letters B, vol 266, no 3-4, pp 419-424, 1991

[25] C T Hill, M A Luty, and E A Paschos, "Electroweak symmetry breaking by fourth-generation condensates and the neutrino spectrum," Phys Rev D, vol 43, pp 3011-3025, May 1991

[26] W J Marcıano, "Exotıc new quarks and dynamıcal symmetry breakıng," Phys Rev D, vol 21, pp 2425 2428, Apr 1980

[27] M E Peskın and T Takeuchı, "Estımatıon of oblique electroweak correctıons," Phys Rev D, vol 46, pp 381 409, Jul 1992

[28] S Weinberg, "Implicatıons of dynamical symmetry breakıng," Phys Rev D, vol $13, \mathrm{p} 974996,1976$ 
[29] S. Dimopoulos and L. Susskind, "Mass without scalars," Nuclear Physics B, vol, 155, no. 1, pp. 237-252, 1979.

[30] T. Appelquist and L. C. R. Wijewardhana, "Chiral hierarchies from slowly running couplings in technicolor theories," Phys. Rev. D, vol. 36, pp. 568-580, Jul 1987.

[31] M. Piai, "Lectures on walking technicolor, holography and gauge/gravity dualities," Adv. High Energy Phys., vol. 2010, p. 464302, 2010, 1004.0176.

[32] O. Cremonesi, "Neutrino masses and Neutrinoless Double Beta Decay: Status and expectations," 2010, 1002.1437.

[33] M. Kamionkowski, "Dark Matter and Dark Energy," 2007, 0706.2986.

[34] J. L. Feng, "Dark Matter Candidates from Particle Physics and Methods of Detection," Ann. Rev. Astron. Astrophys., vol. 48, p. 495, 2010, 1003.0904.

[35] D. Hooper and C. Kelso, "Implications of CoGeNT's New Results For Dark Matter," 2011, 1106.1066.

[36] M. Gabella, "The Randall-Sundrum Model," 2006. [http://www-thphys.physics.ox.ac.uk/people/MaximeGabella/rs.pdf].

[37] Y.-b. Kim, C. O. Lee, I.-b. Lee, and J.-J. Lee, "Brane world of warp geometry: An introductory review," J. Kor. Astron. Soc., vol. 37, pp. 1-14, 2004, hep-th/0307023.

[38] L. Randall and M. D. Schwartz, "Quantum field theory and unification in AdS5," JHEP, vol. 11, p. 003, 2001, hep-th/0108114.

[39] T. Gherghetta, "Warped models and holography," 2006, hep-ph/0601213. 
[40] R. Sundrum, "To the filth dimension and back. (TASI 2004)," 2005, hep-th/0508134.

[41] L. Randall and R. Sundrum, "An alternative to compactification," Phys. Rev. Lett., vol. 83, pp. 4690-4693, 1999, hep-th/9906064.

[42] C. Csaki, J. Erlich, and J. Terning, "The effective Lagrangian in the Randall-Sundrum model and electroweak physics," Phys. Rev., vol. D66, p. 064021, 2002, hep-ph/0203034.

[43] Y. Grossman and M. Neubert, "Neutrino masses and mixings in non-factorizable geometry," Phys. Lett., vol. B474, pp. 361-371, 2000, hep-ph/9912408.

[44] H. Davoudiasl, J. L. Hewett, and T. G. Rizzo, "Experimental probes of localized gravity: On and off the wall," Phys. Rev., vol. D63, p. 075004, 2001, hep-ph/0006041.

[45] N. Arkani-Hamed, M. Porrati, and L. Randall, "Holography and phenomenology," JHEP, vol. 08, p. 017, 2001, hep-th/0012148.

[46] R. Contino, Y. Nomura, and A. Pomarol, "Higgs as a holographic pseudo-Goldstone boson," Nucl. Phys, vol. B671, pp. 148-174, 2003, hep-ph/0306259.

[47] R. Contino, "A holographic composite Higgs model," 2006, hep-ph/0609148.

[48] K. Agashe and R. Contino, "The minimal composite Higgs model and electroweak precision tests," Nucl. Phys., vol. B742, pp. 59-85, 2006, hep-ph/0510164. 
[49] M S Carena, E Ponton, J Santıago, and C E M Wagner, "Electroweak constraints on warped models with custodial symmetry," Phys Rev, vol D76, p 035006, 2007, hep-ph/0701055

[50] M Carena, A D Medina, B Panes, N R Shah, and C E M Wagner, "Collider phenomenology of gauge-higgs unification scenarıs in warped extra dımensions," Phys Rev D, vol 77 p 076003, Apr 2008

[51] H Davoudıasl, S Gopalakrıshna, E Ponton, and J Santıago, "Warped 5-Dimensional Models Phenomenological Status and Experimental Prospects," New J Phys, vol 12, p 075011, 201009081968

[52] M Carena, A D Medına, N R Shah, and C E M Wagner, "Gauge-higgs unification, neutrino masses, and dark matter in warped extra dimensions," Phys Rev D, vol 79, p 096010 May 2009

[53] A Falkowskı, "About the holographıc pseudo-Goldstone boson," Phys Rev, vol D75, p 025017, 2007, hep-ph/0610336

[54] M S Carena, E Ponton, J Santiago, and C E M Wagner, "Light Kaluza-Kleın states in Randall-Sundrum models with custodial SU(2)," Nucl Phys, vol B759, pp 202 227, 2006, hep-ph/0607106

[55] Y Hosotanı, "Dynamıcal Electroweak Symmetry Breakıng in $S O(5) \times U(1)$ Gauge-Higgs Unıfication in the Randall-Sundrum Warped Space," 2009, 09012415

[56] Y Hosotanı, M Tanaka, and N Uekusa, "Collider signatures of the $\mathrm{SO}(5) \mathrm{x}$ U(1) gauge-Higgs unification' 201111036076 
[57] S. R. Coleman and E. J. Weinberg, "Radiative Corrections as the Origin of Spontaneous Symmetry Breaking," Phys. Rev., vol. D7, pp. 1888-1910, 1973.

[58] B. et al., "The International Linear Collider: Gateway to the Quantum Universe," 2007. (http://media.linearcollider.org/gateway/).

[59] E. Brau, James et al., "ILC Reference Design Report: ILC Global Design Effort and World Wide Study," 2007, 0712.1950.

[60] C. S. Team, "A $3 \mathrm{TeV} e^{+} e^{-}$Linear Collider Based on CLIC Technology," 2000. CERN-2000-008. (http://cdsweb.cern.ch/).

[61] Culham, J. R., "Bessel Functions of the First and Second Kind." http://www.mhtl.uwaterloo.ca/courses/me755/web_chap4.pdf, August 2011.

[62] Weisstein, Eric W., "Wolfram MathWorld." http://mathworld.wolfram.com/, August 2011.

[63] Maplesoft, "Maple Help Center." http://www.maplesoft.com/support/help/Maple/, August 2011.

[64] K. Agashe et al., "LHC Signals for Coset Electroweak Gauge Bosons in Warped/Composite PGB Higgs Models," Phys. Rev., vol. D81, p. 096002, 2010, 0911.0059 . 